\title{
Synthesis, characterisation and sensor-functionalisation of transmembrane $\beta$-peptides
}

\author{
Dissertation \\ zur Erlangung des mathematisch-naturwissenschaftlichen Doktorgrades \\ "Doctor rerum naturalium" \\ der Georg-August-Universität Göttingen \\ im Promotionsprogramm Chemie \\ der Georg-August University School of Science (GAUSS) \\ vorgelegt von \\ Denis Pahlke \\ aus Berlin
}

Göttingen, 2018 

Thesis Committee

Prof. Dr. Ulf Diederichsen

Institute of Organic und Biomolecular Chemistry, Georg-August-University Göttingen

Prof. Dr. Claudia Steinem

Institute of Organic und Biomolecular Chemistry, Georg-August-University Göttingen

Members of the Examination Commission

Referent

Prof. Dr. Ulf Diederichsen

Institute of Organic und Biomolecular Chemistry, Georg-August-University Göttingen

Co-Referent

Prof. Dr. Claudia Steinem

Institute of Organic und Biomolecular Chemistry, Georg-August-University Göttingen

Additional Members of the Examination Commission

Prof. Dr. Jörg Enderlein

III. Physical Institute,

Georg-August-University Göttingen

Prof. Dr. Kai Tittmann

Institute of Molecular Enzymology,

Georg-August-University Göttingen

Dr. Franziska Thomas

Institute of Organic und Biomolecular Chemistry, Georg-August-University Göttingen

Dr. Holm Frauendorf

Institute of Organic und Biomolecular Chemistry, Georg-August-University Göttingen

Day of the Oral Examination

13.12.2018 
The work presented in this doctoral thesis has been carried out under the guidance and supervision of Prof. Dr Ulf Diederichsen at the Institute for Organic and Biomolecular Chemistry of the Georg-August-University Göttingen between September 2014 and November 2018.

This work was supported by the Deutsche Forschungsgemeinschaft within the Collaborative Research Center 803 (SFB 803) "Functionality controlled by organization in and between membranes". 


\section{Table of contents}

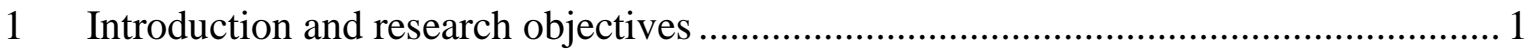

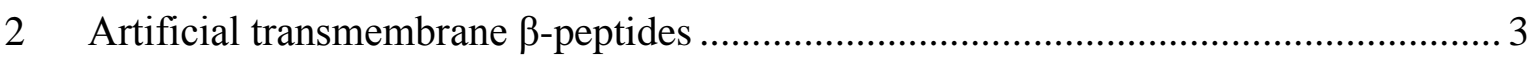

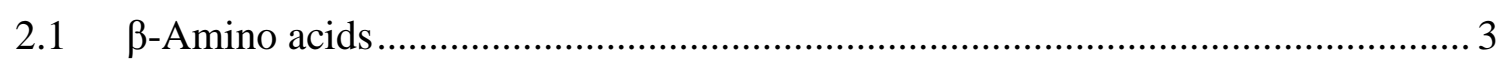

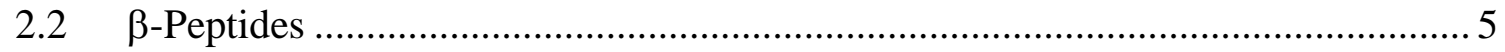

2.3 Transmembrane $\alpha$-peptides as model structures ............................................. 7

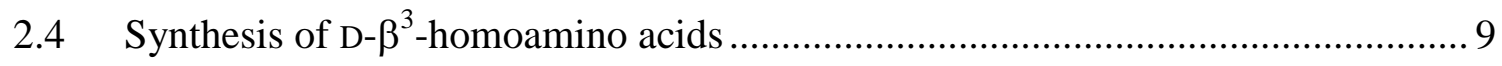

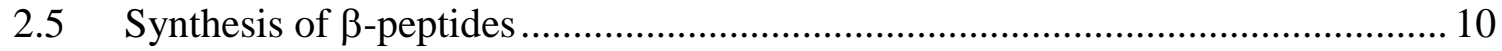

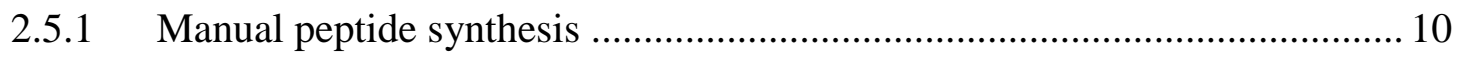

2.5.2 Automatic microwave-supported peptide synthesis ................................... 11

3 Transmembrane $\beta$-peptides functionalised with fluorescence and IR-sensors............ 17

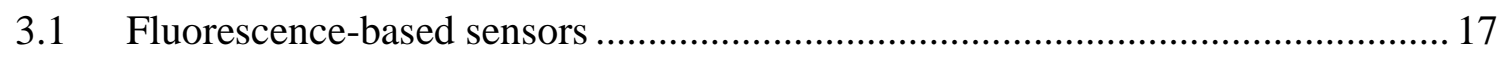

3.2 Design of tryptophan incorporated model $\beta$-peptides ....................................... 19

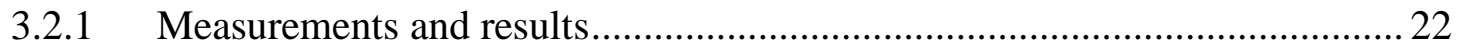

3.2.2 Distance measurements by fluorescence quenching ................................. 28

3.3 Cyanide-labelled $\beta^{3}$-amino acid as molecular sensor for surface-enhanced infrared

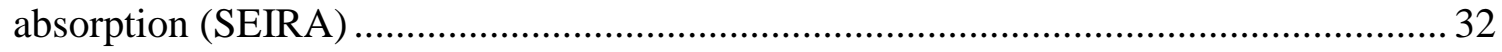

3.3.1 Surface-enhanced infrared absorption (SEIRA) ........................................ 32

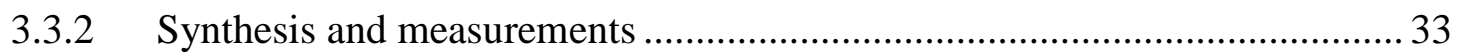

4 Transmembrane $\beta$-peptides as anchor for near-membrane ion-sensors to study the

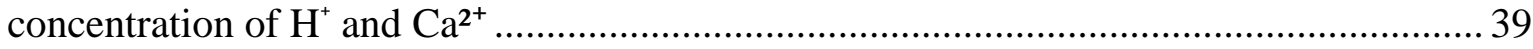

4.1 Near-membrane $\mathrm{H}^{+}$-sensor anchored by transmembrane $\beta$-peptides................... 39

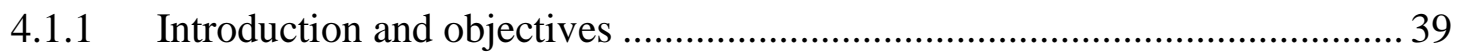

4.1.2 $\mathrm{H}^{+}$Behaviour at the membrane interface ................................................... 41

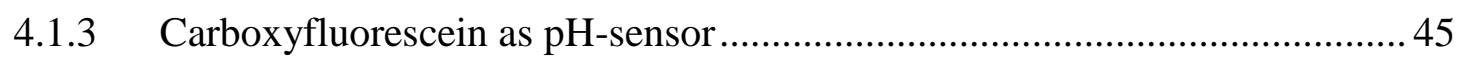

4.1.4 Considerations, synthesis and measurements ............................................. 48

4.1.5 Outlook for Pep 13 and Pep 14................................................................ 58

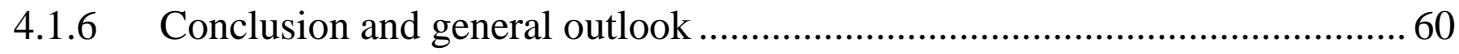

$4.2 \beta$-Peptide functionalisation with SPPS-compatible $\mathrm{Ca}^{2+}$-sensors......................... 62

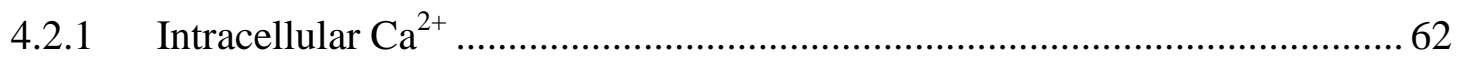

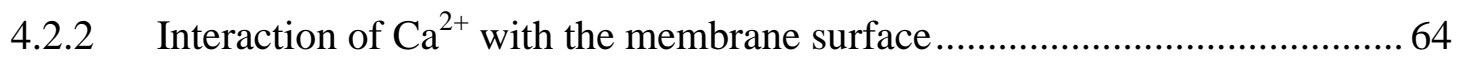

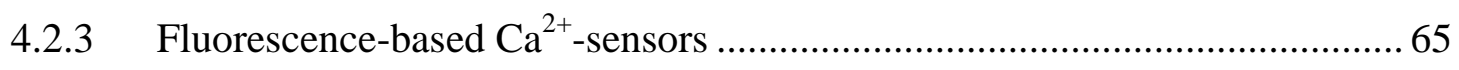


4.2.4 Design strategies of new near-membrane $\mathrm{Ca}^{2+}$-sensors ............................. 71

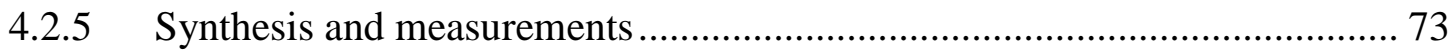

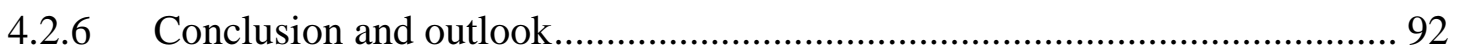

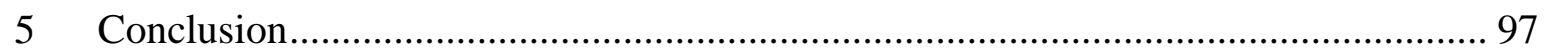

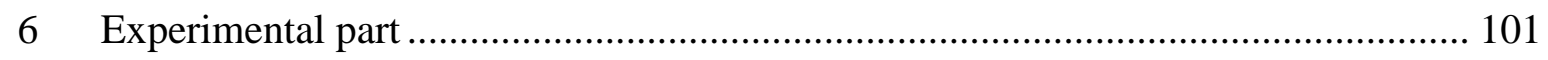

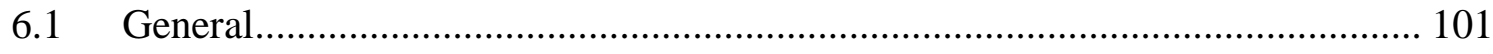

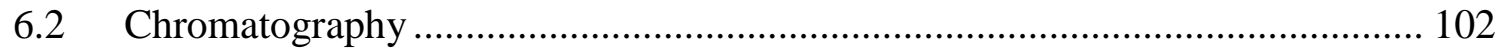

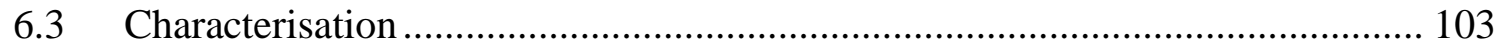

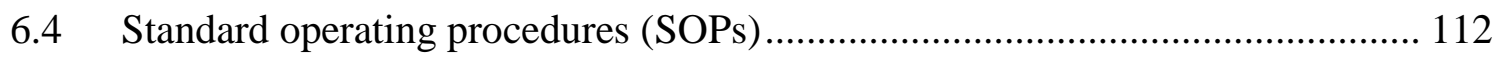

SOP 1 General synthesis of $N$-protected D- $\beta^{3}$-amino acids ............................... 112

SOP 2 Manual loading of the resin with the first amino acid................................ 113

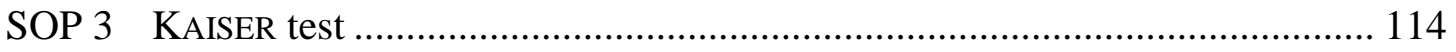

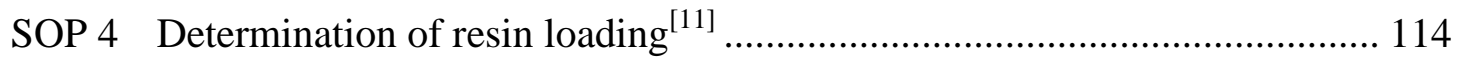

SOP 5 Manual microwave-supported SPPS …...................................................... 114

SOP 6 Automatic microwave-supported SPPS ................................................ 115

SOP 7 Preparation of large unilamellar vesicles (LUV) ...................................... 116

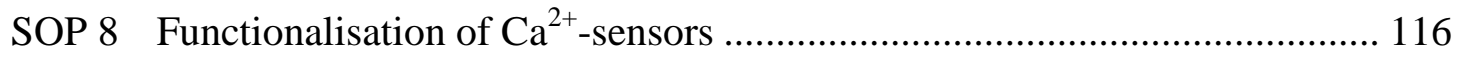

SOP 9 Labelling of $\beta$-peptides with carboxyfluorescein as $\mathrm{pH}$-sensor .................. 117

SOP 10 Labelling of $\beta$-peptides with $\mathrm{Ca}^{2+}$-sensors 38 and $46 \ldots \ldots \ldots \ldots \ldots \ldots \ldots \ldots \ldots . . . . . . . . . . .117$

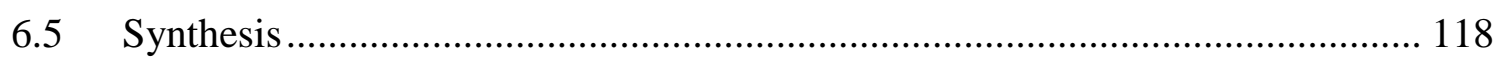

6.5.1 $\quad \beta^{3}$-Homoamino acids and coupling reagent DMT/NMM/TOS .................. 118

6.5.2 2-[2'-(2’'-Aminophenoxy)ethoxy]-5-(benzyloxy)aniline........................... 125

6.5.3 Base-labile BAPTA-based $\mathrm{Ca}^{2+}$-sensor ................................................... 130

6.5.4 Acid-labile BAPTA-based $\mathrm{Ca}^{2+}$-sensor .................................................. 136

6.5.5 Acid-labile MOBHA-based $\mathrm{Ca}^{2+}{ }^{2}$-sensor ................................................. 141

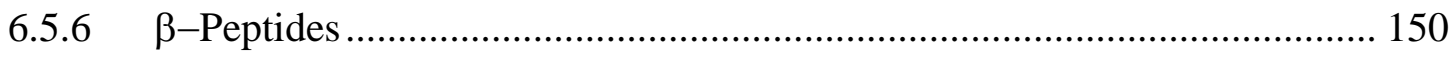

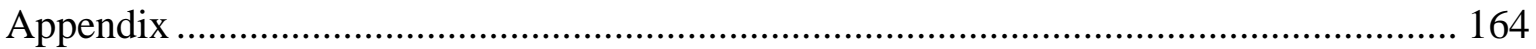

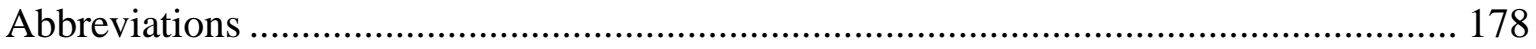

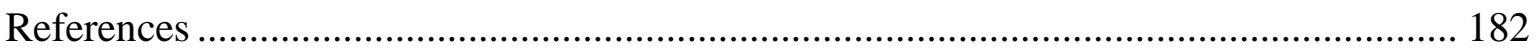

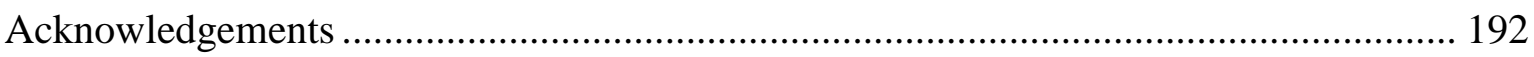






\section{Introduction and research objectives}

Peptides and proteins are important components for living organisms. They fulfil a various number of tasks ranging from structure-giving functions up to transporting and catalysing processes. ${ }^{[1]}$ Membrane proteins represent an important class of proteins as they are encoded in up to $30 \%$ of the open reading frame. More than $50 \%$ of currently available drugs target membrane proteins, emphasising the importance of membrane protein research. ${ }^{[2,3]}$ Membrane proteins usually feature a complex structure, and due to their hydrophobic properties, the isolation and purification of membrane proteins can be challenging. Nevertheless, a large number of complex membrane proteins consist of the well-known $\alpha$-helices. ${ }^{[4,5]}$ Therefore, different peptidomimetics, like the WALP and KALP model peptides, have been developed to imitate the behaviour and function of $\alpha$-helical transmembrane proteins in a less complex environment. ${ }^{[6-8]}$ Such peptidomimetic model systems help to investigate the structure and dynamics of transmembrane peptides within lipid bilayers. Thus, they offer the possibility to understand protein-protein binding, signal transduction, protein stability and enzyme catalysis in a variety of cellular processes better. ${ }^{[1,6-8]}$ Besides the use of transmembrane $\alpha$-peptides, the design and synthesis of further transmembrane model systems based on artificial $\beta$-peptides consisting of $\beta$-amino acids has become of great interest. ${ }^{[9]} \beta$-Amino acids differ from the natural $\alpha$-amino acids by an additional methylene group in the amino acid backbone, and due to this modification, $\beta$-peptides are able to form more stable secondary structures than $\alpha$-peptides. ${ }^{[9,10]}$ Peptidomimetics based on $\beta$-amino acids are still widely unexplored. The development of model transmembrane $\beta$-peptides, however, can help to understand the orientation or the peptide aggregation of helical transmembrane peptides and proteins. ${ }^{[11-15]}$

The first part of this thesis focuses on the synthesis, characterisation and investigation of transmembrane $\beta$-peptides which were inspired by prior studies. ${ }^{[12]}$ These novel transmembrane $\beta$-peptides differ in the $N$-terminal modification and partially in the peptide length, which extend range of transmembrane $\beta$-peptides. ${ }^{[7,14]}$ Their secondary structure was analysed in organic solvents as well as in large unilamellar vesicles by means of circular dichroism spectroscopy. Furthermore, membrane insertion was investigated by tryptophan fluorescence spectroscopy. ${ }^{[16-21]}$ Therefore, D- $\beta^{3}$-homotryptophan (D- $\beta^{3}$-hTrp) was inserted either peripherally or centrally into the hydrophobic peptide sequence to observe the tryptophan fluorescence which is influenced by the surrounding polarity. ${ }^{[16-21]}$ Furthermore, the determination of the exact position of the D- $\beta^{3}-h$ Trp in the membrane was 
examined by tryptophan fluorescence quenching experiments based on DOXYL-labelled lipids. ${ }^{[22-25]}$ In order to perform surface-enhanced infrared absorption (SEIRA) spectroscopy to obtain information about orientation, association and localisation of the $\beta$-peptides within the membrane, the novel $\beta$-amino acid Fmoc-D- $\beta^{3}-\mathrm{Ala}(\mathrm{CN})-\mathrm{OH}(\mathbf{1})$ was synthesised and incorporated into the transmembrane $\beta$-peptide sequence. ${ }^{[26-28]}$ These experiments were performed in collaboration with STAFFA from the HILDEBRANDT research group.

The second part of this thesis deals with the question whether $\beta$-peptides can function as membrane-anchored molecular rulers to investigate processes at the membrane interface. Already known sensors were anchored by acyl chains or lipid molecules into the membrane. ${ }^{[29-37]}$ These kinds of molecules limit the exact determination of the sensor distance to the membrane surface as they have no fixed rigid anchoring structure. Hence, the advantages of $\beta$-peptides like conformationally well-defined and stable secondary helix structures seem to be interesting for the development of new transmembrane anchoring peptides. ${ }^{[10]}$ A recently published transmembrane $\beta$-peptide consists of a hydrophobic inner membrane part that is flanked by two D- $\beta^{3}$-hTrp residues at each side of the peptide. ${ }^{[38]}$ These residues anchor the peptide in the membrane and stabilise the vertical insertion of the $\beta$-peptide. Based on this basic structure, transmembrane $\beta$-peptides were equipped either with a $\mathrm{pH}$-sensor (carboxyfluorescein) or with a newly designed $\mathrm{Ca}^{2+}$-sensor. To analyse near-membrane $\mathrm{pH}$ values, two carboxyfluorescein-labelled transmembrane $\beta$-peptides were synthesised with different sensor distances to the membrane surface and $\mathrm{pH}$ titration was employed. The different distances were achieved by attaching the sensor to peptides with varying lengths of the peptide sequence outside the membrane. To analyse near-membrane $\mathrm{Ca}^{2+}$ concentration, two $\mathrm{Ca}^{2+}$-sensors were developed based on literature known models. ${ }^{[37]}$ Titration series with the sensors attached to hydrophilic peptides and $\mathrm{Ca}^{2+}$ were performed and analysed with UV/Vis and fluorescence spectroscopy to investigate how the fluorescent properties of the sensors depend on the $\mathrm{Ca}^{2+}$ concentration. Afterwards, $\mathrm{Ca}^{2+}$ concentration differences were applied to study one of the sensors attached to transmembrane $\beta$-peptides. 


\section{Artificial transmembrane $\beta$-peptides}

The peptide synthesis is based on the stepwise linking of monomers in a solution phase or on a solid phase. During the last decades a peptidomimetic with an increasing potential is the $\beta$-peptide. In the following the synthesis of $D-\beta^{3}$-homoamino acids as SPPS building blocks and the $\beta$-peptide synthesis are shown.

\section{$2.1 \beta$-Amino acids}

Native peptides and proteins consist of $\alpha$-amino acids. These $\alpha$-amino acids exhibit a common structure, which includes an amine functionality, a carboxylic acid functionality and a characteristic side chain. All of them are connected to a central $\alpha-\mathrm{C}$ atom (see Figure $2.1)^{[1,39]}$

a)<smiles>[R]C(N)C(=O)O</smiles>

b)<smiles>[R]C(CN)C(=O)O</smiles>

c)<smiles>[R]C(N)CC(=O)O</smiles>

d)<smiles>[R][R](C(=O)O)C([R])N</smiles>

Figure 2.1 a) General structure of $\alpha$-amino acids. Based on the incorporation of an additional methylene group in the $\alpha$-amino acid backbone different substitutions in $\beta$ - amino acids are possible: b) $\beta^{2}$-amino acid, c) $\beta^{3}$-amino acid, d) $\beta^{2,3}$-amino acid. ${ }^{[10]}$ Adapted with permission from R. P. Cheng et al., Chem. Rev. 2001, 101, 3219-3232. Copyright (2001) American Chemical Society.

The $\beta$-amino acids can be classified into homoamino and isoamino acids. The $\beta$-isoamino acids are based on the structure of the $\alpha$-amino acids, except for the amino group, which is moved without another change to the $\beta$-carbon atom of the side chain. ${ }^{[40]}$ Due to this, the side chain is reduced by one methylene group and the backbone is increased by one. The $\beta$-alanine, found in the coenzyme $A$, follows this nomenclature. ${ }^{[41]}$ The $\beta$-homoamino acids differ from the natural $\alpha$-amino acids by the incorporation of an additional methylene group in the backbone. This results in an additional torsional angle (see Figure 2.4 a) as well as a second position for a side chain linkage (see Figure 2.1) without any functional reduction on the side chain group. ${ }^{[9,10,40,42]}$ In the following, $\beta$-homoamino acids are referred to as $\beta-\mathrm{hXxx}(\mathrm{Xxx}=$ three letter code), a designation that is based on the nomenclature of the $\alpha$-amino acids. The numbering of the $C$-atoms begins with the $C$-atom of the carboxylic acid and ends at the $C$-atom next to the amine group (see Figure 2.1). The designation with Greek letters starts at C-2..$^{[9,10,40,43]}$ Furthermore, the side chain can be bound to the $C-2, C-3$ or both. Hence, these amino acids are called $\beta^{2}-, \beta^{3}$ - or $\beta^{2,3}$-amino acids (see Figure 2.1). A multiple substitution at C-2 and C-3 with side chain groups is also possible and leads for example to $\beta^{2,2}-, \beta^{3,3}$ - or $\beta^{2,2,3,3}$-amino acids. ${ }^{[9]}$ 
Despite the great potential for modifying $\beta$-amino acids, the occurrence of $\beta$-amino acids in nature is low. They are found, for example, as free amino acids or as special building blocks in peptides and alkaloids. ${ }^{[9,44]}$ The low abundance of $\beta$-peptides might be because of a limited availability in the origins of life, structurally disadvantages compared to $\alpha$-peptides and differences in reactivity of the monomers. Due to the additional methylene group in $\beta$-amino acids, the reactivity of $\alpha$ - and $\beta$-amino acids are different. Phosphorylation reactions, which are important for the regulation of biological processes, provide a method to examine different reaction behaviours. ${ }^{[45]}$ Studies of $N$-phosphoryl $\alpha$-aspartic acid showed that the $\alpha$-carboxylic acid displays a different behaviour compared to the $\beta$-carboxylic acid. ${ }^{[46,47]}$ For example, dipeptides are only formed between $\alpha$-amino acids which results in an $\alpha$-dipeptide (see Figure 2.2 ). ${ }^{[47]}$
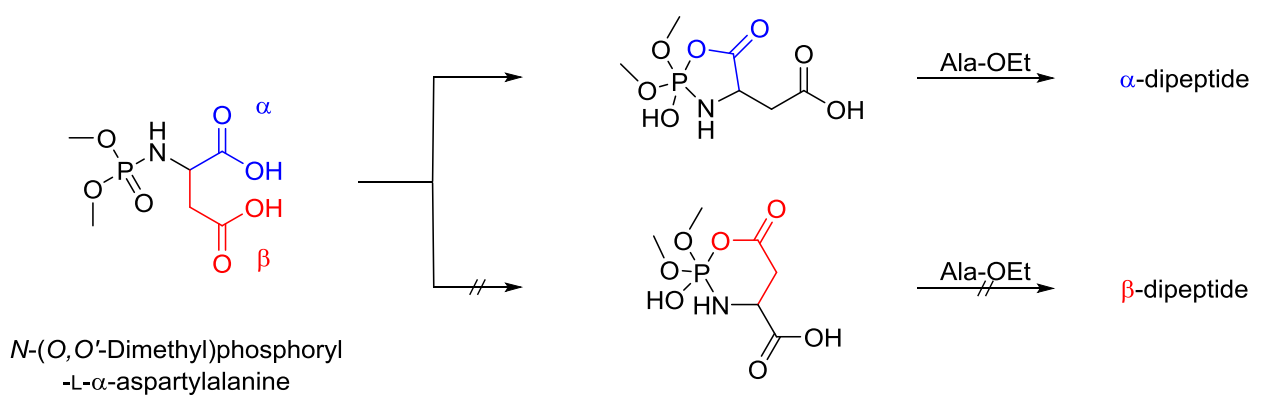

Figure 2.2 Possible intermediates for the formation of $\alpha$ - or $\beta$-dipeptides by $N$-phosphorylamino acid. ${ }^{[47]}$ Adapted with permission from Z.-Z. Chen et al., J. Org. Chem. 2003, 68, 4052-4058. Copyright (2003) American Chemical Society.

Thus, it is presumed that the $\alpha$-carboxylic acid has a higher activity after $N$-phosphorylation then the $\beta$-carboxylic acid. Comparable results were found in a nitrosation reaction with amino acids, a reaction that was observed in preserved food within the stomach. ${ }^{[48,49]}$ Here, a similar reactivity of amino acid types was found ( $\alpha$-amino acids $<\beta$-amino acids $<\gamma$-amino acids). Besides other explanations, these different reactivities are an indication why $\alpha$-amino acids are preferred in natural systems. ${ }^{[46,47]}$ However, during the last decades the research on $\beta$-amino acids has become more important. Especially SEEBACH et al. and GELLMAN et al. published ground-breaking work in this research field. ${ }^{[9,10,40,50-54]}$ The incorporation of $\beta$-amino acids in $\alpha$-peptides and the synthesis of pure $\beta$-peptides lead to interesting compounds. Properties in connection with biological systems, structure and thermal stability have been described extensively. ${ }^{[9,55-59]}$ SEEBACH et al. pointed out that the enzymatic proteolysis of mixed and pure $\beta$-peptides is slower than the proteolysis of native amino acid bonds or it is totally blocked. ${ }^{[60]}$ 
Due to the widespread use as peptidomimetics, the synthesis of the monomeric building blocks and the optimisation of the SPPS based peptide synthesis represent important research fields.

\section{$2.2 \quad \beta$-Peptides}

A lot of basic research on the secondary structure was performed with regard to the behaviour of $\beta$-amino acids and the resulting $\beta$-peptides. In general, $\beta$-peptides can mimic the native $\alpha$-helices, the sheet structure as well as the hairpin structures. ${ }^{[9]}$ Furthermore, $\beta$-peptides can form different helices and the type of helix can be anticipated by the type of amino acid side chain and the substitution of the $\beta$-amino acid. ${ }^{[9]}$ The helix is defined by the number of atoms between the hydrogen bond, formed by the backbone $\mathrm{NH}$ and $\mathrm{O}$ (see Figure 2.3)..$^{[9,10,43,61]}$ The best studies helices are the 8-, 10-, 12-, 10/12- and 14-helix.
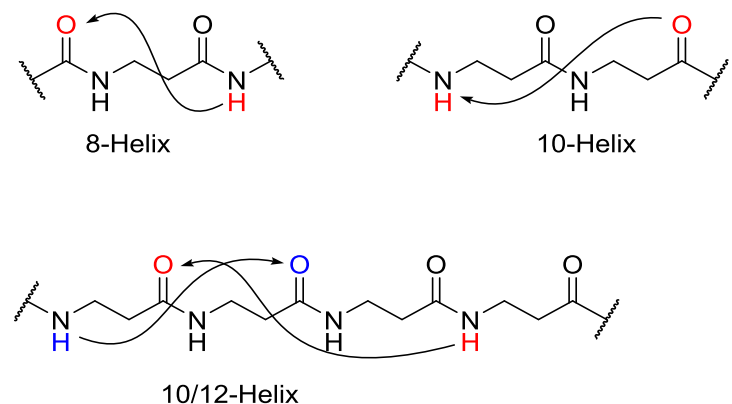
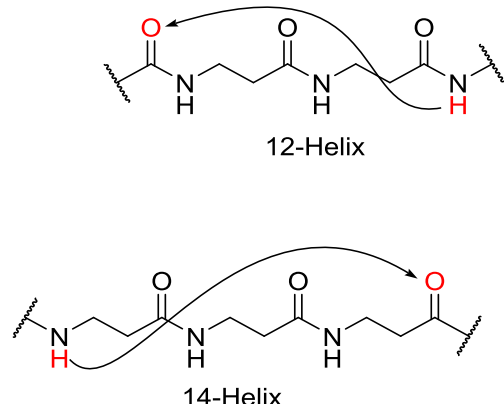

Figure 2.3 Hydrogen bond-based nomenclature of $\beta$-peptides. The atoms involved in hydrogen bonding are coloured. ${ }^{[10]}$ Adapted with permission from R. P. Cheng et al., Chem. Rev. 2001, 101, 3219-3232. Copyright (2001) American Chemical Society.

Due to the easier accessibility of the $\beta^{3}$-amino acid monomers, compared with other $\beta$-amino acids, the secondary structure formed by $\beta^{3}$-peptides is well known. ${ }^{[9]}$ The conformation variation in the $\mathrm{C}_{\alpha}-\mathrm{C}_{\beta}$ bond of these peptides prefers the formation of helices or turns. Possible conformations are the trans conformation $\left(\theta=180^{\circ}\right)$, gauche conformation $\left(\theta= \pm 60^{\circ}\right)$, skew conformation $\left(\theta= \pm 88^{\circ}\right)$ and syn-clinal conformation $\left(\theta= \pm 48^{\circ}\right)$. The gauche conformation is the favourable conformation for $\beta^{3}$-peptides because of steric effects which result from repulsive forces (see Figure $2.4 \mathrm{~b}$ ). ${ }^{[9]}$ The most common secondary structures are the 12- and 14-helix. Other helix conformations, for example the 10/12-helix, can be generated by a combination of $\beta^{2} / \beta^{3}$-amino acids. ${ }^{[62]}$ 
a)

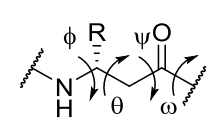

b)

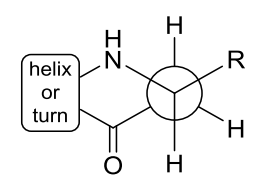

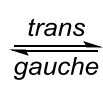

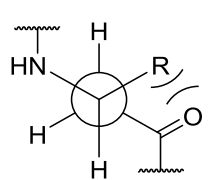

Figure 2.4 a) Torsion angles in $\beta$-peptides. b) Different conformations of $\beta^{3}$-peptides. The favoured conformation is the gauche conformation and it results in the formation of a helix or a turn ${ }^{[9,10,43]}$ Adapted with permission from R. P. Cheng et al., Chem. Rev. 2001, 101, 3219-3232. Copyright (2001) American Chemical Society.

The helices differ in their characteristics such as radius, residue per turn or rise per residue. ${ }^{[43]}$ These helical structures were investigated by spectroscopy methods like $\mathrm{NMR},{ }^{[63]} \mathrm{CD}^{[64-70]}$ and X-ray crystallography. ${ }^{[63]}$ For similar types of helices, these different methods lead to virtually the same results. There can be small variations in the structure based on the measured differences for example in probe preparation or in the used model peptides. In Table 2.1, characteristic structure properties of the 12- and 14-helix based on simulation and X-ray measurements are given. In general, the thinner 12-helix differs with 2.5 residue per turn from the 14-helix, which exhibits 3.0 residue per turn. ${ }^{[10]}$ These averaged residue per turn of the 12-helix and 14-helix allow a simpler design of the peptide motifs as compared to the $\alpha$-peptides (3.6 residue per turn). ${ }^{[71-73]}$ Consequently, the 12-helix has a higher rise per residue $(2.1 \AA)$ than the 14-helix with $1.56 \AA .^{[10]}$ As with the $\alpha$-peptides, it is possible to control the handedness of the presented helix by selecting the chirality of the amino acids used. $\alpha$-Amino acids with a natural chirality (L-amino acids) induce a right-handed $\alpha$-helix. ${ }^{[1]}$ The right-handed 14-helix can be achieved by the use of D- $\beta$-amino acids. In the case of a 12-helix, a left-handed helix is induced by the use of D- $\beta$-amino acids. ${ }^{[10,11,40,74-77]}$

Table 2.1 The characteristic structural properties of 12-helices and 14-helices based on NMR, CD and X-ray crystallography of model peptides. There are small variations in the structure values based on different literatures.

\begin{tabular}{|cc|cccc|}
\hline & Residues/turn & Rise/turn $(\AA)$ & Rise/residue $(\AA)$ & Radius $_{(\AA)}$ & Lit. \\
\hline Simulation $_{\text {12-helix }}$ & 2.50 & - & - & 2.3 & {$[10]$} \\
& $2.56,2.58$ & - & $2.05,2.1$ & - & {$[78]$} \\
\hline Crystal $_{12-\text { helix }}$ & 2.7 & 5.4 & 2.0 & 2.1 & {$[79]$} \\
\hline \multirow{2}{*}{ Simulation $_{\text {14-helix }}$} & 3.0 & - & 1.56 & 2.7 & {$[10]$} \\
& 3.25 & - & 1.56 & - & {$[80]$} \\
& - & 4.69 & - & 2.6 & {$[81]$} \\
\hline Crystal $_{\text {14-helix }}$ & 3.1 & - & - & - & {$[82]$} \\
\hline
\end{tabular}


The helix formation can be predicted by the chosen amino acid sequence and the choice of amino acids. Cyclic amino acids, for example, stabilise different helices due to the ring conformation (see Figure 2.5). ${ }^{[10,43,51]}$

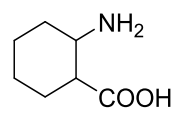

14-helix (hydrophobic)

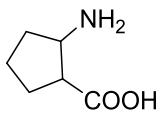

12-helix (hydrophobic)

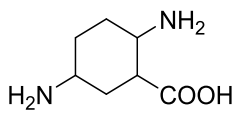

14-helix (hydrophilic)

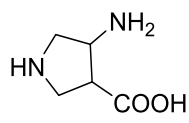

12-helix (hydrophilic)

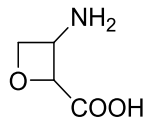

10-helix

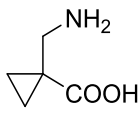

8-helix

Figure 2.5 Cyclic amino acids that support different helix conformations. ${ }^{[10,43,51]}$

The non-cyclic amino acids can influence the secondary structure as well. Here, the type of branching is important. ${ }^{[83]}$ In contrast to molecular-dynamics (MD) simulations of GLÄTTLI et al. ${ }^{[84]}$ the CD measurements of RAGUSE et al. ${ }^{[83]}$ indicate that a high amount of $\beta^{3}-\mathrm{hVal}$ forces the helix in a 14-helix conformation whereas a high amount of $\beta^{3}$-hLeu forces a 12-helix conformation. It is assumed that branching next to the $\beta$ - $C$-atom is favourable for a 14-helix, like in the case of $\beta^{3}$-hVal. Studies of unbranched amino acids implied controversially discussed results. ABELE et al. proved that model peptides with a high amount of $\beta^{3}$-hLys in MeOH show the typical CD spectrum of a 14-helix. ${ }^{[85]}$ In contrast, the same peptides in $\mathrm{H}_{2} \mathrm{O}$ or buffer have no typical pattern, due to a possible disruption of the H-bond network by $\beta^{3}$-hLys. The same peptides with $\beta^{3}$-hSer instead of $\beta^{3}$-hLys show a pattern specific for a 14-helix. For this reason, ABELE et al. assumed a stabilising effect of $\beta^{3}$-hSer in aqueous solutions. ${ }^{[85]}$ KRITZER et al. concluded that in a model peptide the position of the unbranched amino acids $\beta^{3}$-hLys and $\beta^{3}$-hGlu has a stabilising or destabilising effect. ${ }^{[74]}$ Near the $N$-terminus a stabilising effect of $\beta^{3}$-hLys and a destabilising effect of $\beta^{3}$-hGlu was found, whereas at the $C$-terminus a reversed effect was observed.

\subsection{Transmembrane $\alpha$-peptides as model structures}

Biological membranes are the place of essential reactions, transmembrane signalling as well as anchor point of cell structure units. ${ }^{[1,86,87]}$ Therefore, a lot of peptides are localised in or on the lipid membrane. Hydrophobic $\alpha$-helices are a ubiquitous structure motif. This is verified by the fact that $20-25 \%$ of open reading frames in the genome sequence encode these $\alpha$-helices. ${ }^{[4,5]}$ In order to understand how peptides are anchored in the 
membrane, a vast amount of synthetic peptides based on transmembrane $\alpha$-helices has been studied. ${ }^{[7,8]}$ Generally, the model peptides feature a hydrophobic core, which interacts with the lipid acyl chains. This hydrophobic core commonly consists of amino acids with hydrophobic side chains, such as leucine or alanine. The amino acids are either arranged alternatingly or the core features one type of amino acid that is repeated several times. The hydrophobic core is usually flanked by polar amino acids, for example lysine or tryptophan. Lysine increases the solubility of the model peptide ${ }^{[88]}$ and tryptophan ensures that the peptide is anchored in the membrane. ${ }^{[7,89]}$ It has been shown that tryptophan is more efficient in anchoring the peptide than lysine. In Table 2.2, examples of model transmembrane peptides are given.

Table 2.2 Examples of model peptides designed for studying lipid-peptide interactions. ${ }^{[7]}$

\begin{tabular}{|c|c|}
\hline Model peptide & Amino acid sequence \\
\hline WALP & Ac-GWW $(\mathrm{LA})_{m} \mathrm{~L}_{n} \mathrm{WWA}-\mathrm{CONH}_{2}\left({ }_{m}=3,5,6,7,8,10,12,{ }_{n}=0,1\right)$ \\
\hline KALP & $\operatorname{Ac}-\mathrm{GKK}(\mathrm{LA})_{m} \mathrm{LWWA}-\mathrm{CONH}_{2}\left({ }_{m}=6,8,10,12\right)$ \\
\hline $\mathbf{L}_{24}$ & $\mathrm{Ac}-\mathrm{KKL}_{24} \mathrm{KK}-\mathrm{CONH}_{2}$ \\
\hline LW & $\mathrm{Ac}-\mathrm{KKWWL} \mathrm{L}_{8} \mathrm{AL}_{8} \mathrm{WWKK}-\mathrm{CONH}_{2}$ \\
\hline
\end{tabular}

The length of the hydrophobic core especially influences how peptides interact with the lipid membrane. If the hydrophobic core of the peptide is longer than the lipid bilayer, a positive hydrophobic mismatch is generated. A negative hydrophobic mismatch, on the contrary, results if the hydrophobic core of the peptide is shorter than the length of the lipid bilayer (see Figure 2.6). ${ }^{[8]}$

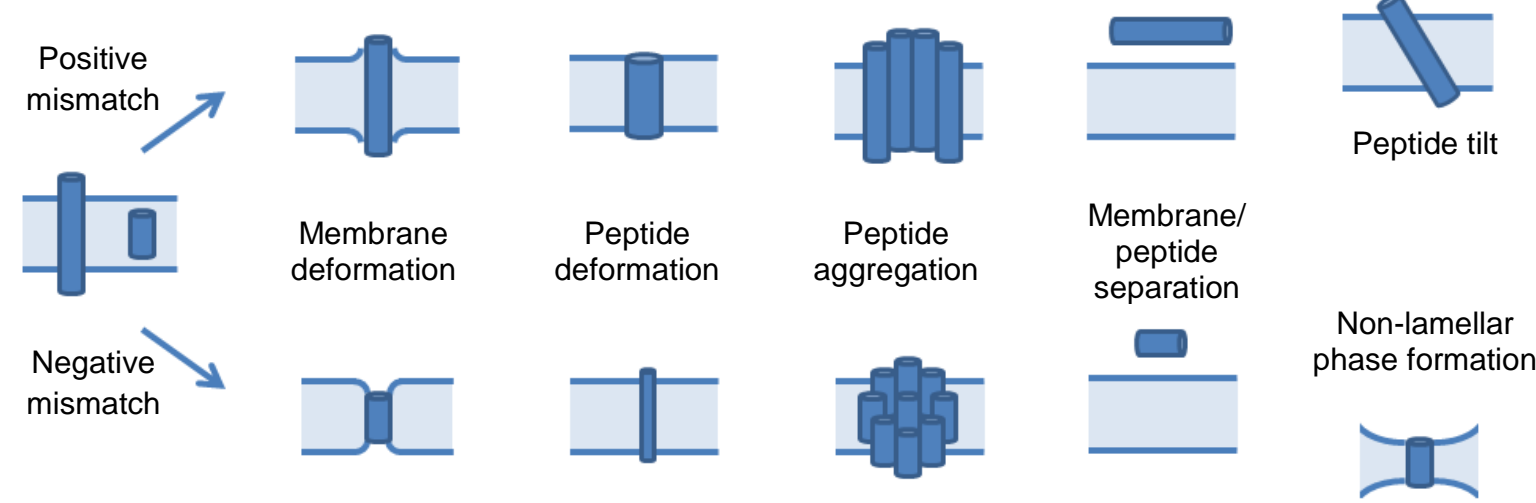

Figure 2.6 Possible lipid-peptide interactions to prevent mismatch situations. ${ }^{[8]}$ Adapted with permission from M. R. R. de Planque et al., Mol. Membr. Biol. 2003, 20, 271-284. Copyright (2003) Taylor \& Francis. 
Depending on the kind of mismatch, the lipid-peptide interaction is different. It can lead to a deformation of the membrane, deformation of the peptide or other interactions to prevent the peptide lipid mismatch (see Figure 2.6). ${ }^{[8]}$

The development of alternative approaches for transmembrane peptides can help to increase the number of membrane study tools. In various studies transmembrane $\beta$-peptides were developed, which are based on the already mentioned KALP and $\mathrm{L}_{24}$ model peptides. ${ }^{[7,11-15]}$ Among others, with these transmembrane $\beta$-peptides incorporation and aggregation studies were performed.

\subsection{Synthesis of $D-\beta^{3}$-homoamino acids}

As starting material for the synthesis of D- $\beta^{3}$-homo amino acids, the respective Fmoc protected $\alpha$-amino acids were used. The free carboxylic acid was treated with isobutyl chloroformate (1.10 eq) and triethylamine (1.10 eq) in THF. This resulted in an activated mixed organic acid anhydride that reacted with diazomethane (2.00 eq) to a diazo ketone (see Figure 2.7). This intermediate can be used without any purification. In the case of a discolouration (red instead of yellow) purification by flash chromatography (pentane/ethyl acetate) is also possible and advisable.

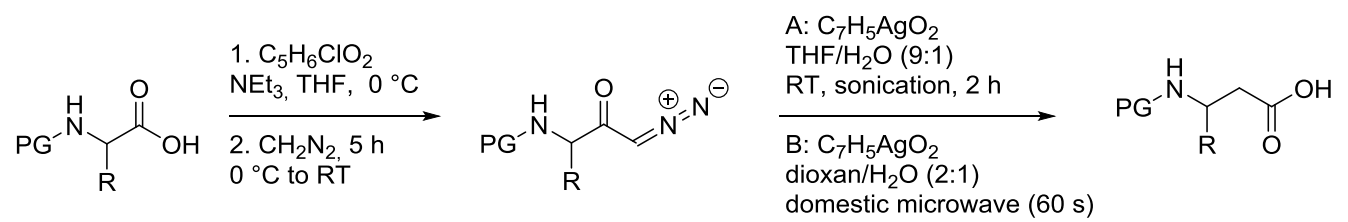

Figure 2.7 Reaction schema of the ARNDT-EISTERT homologation to obtain $\beta^{3}$-homoamino acids $(P G=$ protection group). ${ }^{[11]}$

The second step of the ARNDT-EISTERT homologation was a silver(I) catalysed WOLFF rearrangement. Therefore, two reaction conditions were established. ${ }^{[9,11,90,91]}$ For the first reaction condition, the educt was solved in $\mathrm{THF} / \mathrm{H}_{2} \mathrm{O}(9: 1)$ and silver(I) benzoate $(0.10 \mathrm{eq})$ was added to the solution. This mixture was sonicated for $2 \mathrm{~h}$ at RT. For the second reaction condition, the educt was solved in dioxane $/ \mathrm{H}_{2} \mathrm{O}(2: 1)$ and after the addition of silver(I) benzoate $(0.10 \mathrm{eq})$ the mixture was irradiated in a domestic microwave $(480 \mathrm{~W}$, $60 \mathrm{~s}$ ). Here, a partly incomplete conversion was observed, which is likely due to the used glassware. The thinnest possible wall thickness is probably more beneficial than a thick one. In the following, the reaction was worked up and the product was purified by precipitation in cold pentane or by flash chromatography. Small amounts of impurities remained in the product after purification by precipitation, but they did not affect the following peptide synthesis. 


\subsection{Synthesis of $\beta$-peptides}

\subsubsection{Manual peptide synthesis}

The $\beta$-peptides were synthesised by solid phase peptide synthesis based on the Fmoc strategy. ${ }^{[92,93]}$ The used method is well established. ${ }^{[14,38,94]}$ As an insoluble solid phase a MBHA rink amide or a Nova PEG LL rink amide resin was used. The substrates differ in their swelling properties and loading density. The MBHA rink amide resin is based on polystyrene and was used with a resin loading of $0.3-0.5 \mathrm{mmol} / \mathrm{g}$. Due to the polyethylene glycol (PEG) substrate of the Nova PEG LL rink amide resin, the swelling properties are higher and a smaller resin loading $(0.18 \mathrm{mmol} / \mathrm{g})$ was used, so that this resin was chosen in challenging syntheses. Both resins form a carboxamide group at the $C$-terminal end on account of the rink amide functionalisation. ${ }^{[95]}$ This prevention of charge at the $C$ - and $N$-terminus of transmembrane peptides is very common. ${ }^{[7,8,96,97]}$ The resin was washed during the synthesis with $\mathrm{MeOH}$, (DCM) and DMF. All synthesis steps were supported by microwave radiation which increases the effectiveness of the coupling reaction (see Chapter 6.4). ${ }^{[98-102]}$

The $N$-terminal resin-bound Fmoc group was cleaved twice by using a solution of NMP/piperidine/DBU $(v / v / v ; 79: 20: 1)$. The free $N$-terminus was treated with a mixture consisting of amino acid (5.00 eq), which was solved in a solution of $0.49 \mathrm{M} / 0.50 \mathrm{M}$ HATU/HOAt in DMF (5.00 eq) followed by adding 2.0 M DIPEA in NMP (10.00 eq). Depending on the efficiency of the coupling, this step was performed once, twice or thrice. (see Figure 2.8).

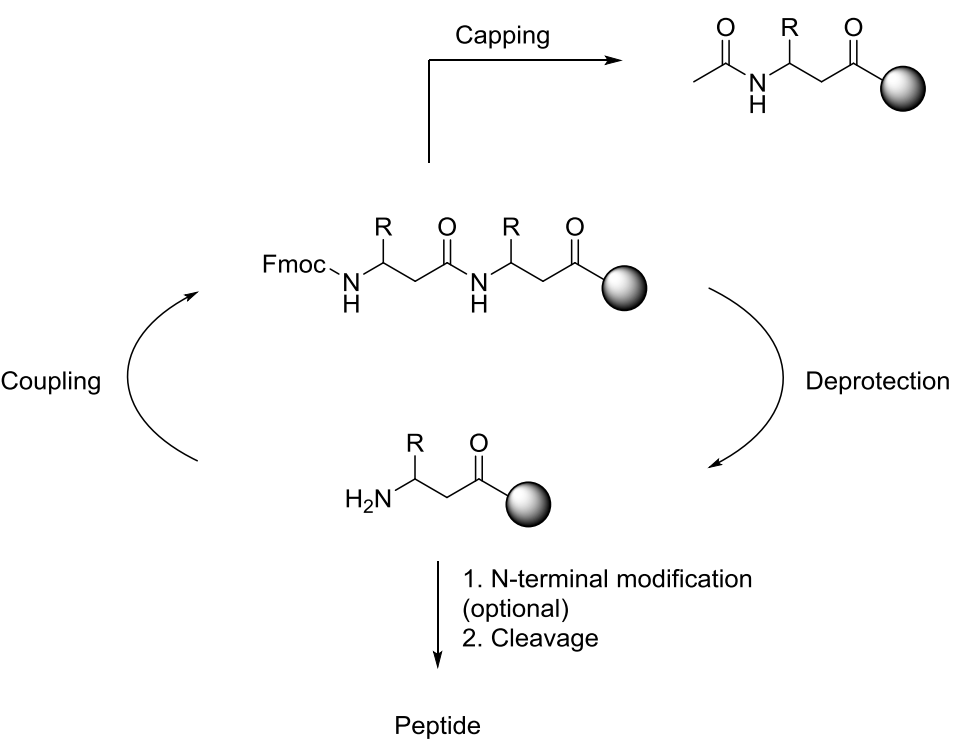

Figure 2.8 Schematic overview of solid phase peptide synthesis. 
The peptides based on $\mathrm{D}-\beta^{3}-\mathrm{hLeu}$ showed an inadequate coupling efficiency, which led, despite a high number of coupling to a small amount of target peptide. Subsequently, the free amino groups were capped with acetic anhydride in NMP $(v / v ; 20: 80)$ to prevent undesirable peptide elongation (see Figure 2.8). The entire resin-bound peptide was dried, cleaved by TFA/ $\mathrm{H}_{2} \mathrm{O} / \mathrm{TIS}(v / v / v ; 95: 2.5: 2.5)$ and purified by HPLC. A modification of the $\mathrm{N}$-terminus with acetylation or fluorophores was performed on resin before cleaving. In the mass spectrometric examination of the synthesised peptides, the tryptophan containing peptides showed temporary mass differences. The Boc protected $\beta^{3}$-hTrp showed a rearrangement during deprotection (see Figure 2.9).

a)

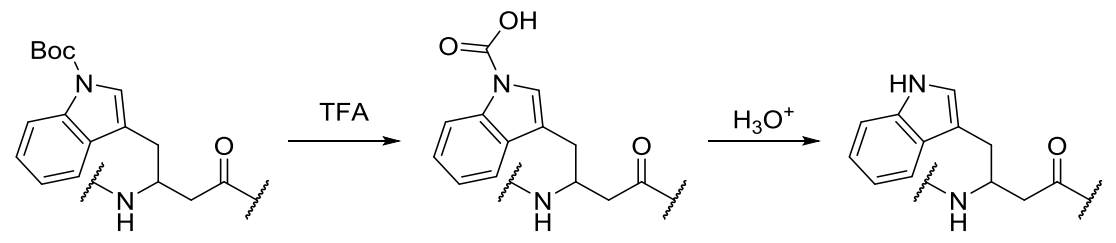

b)
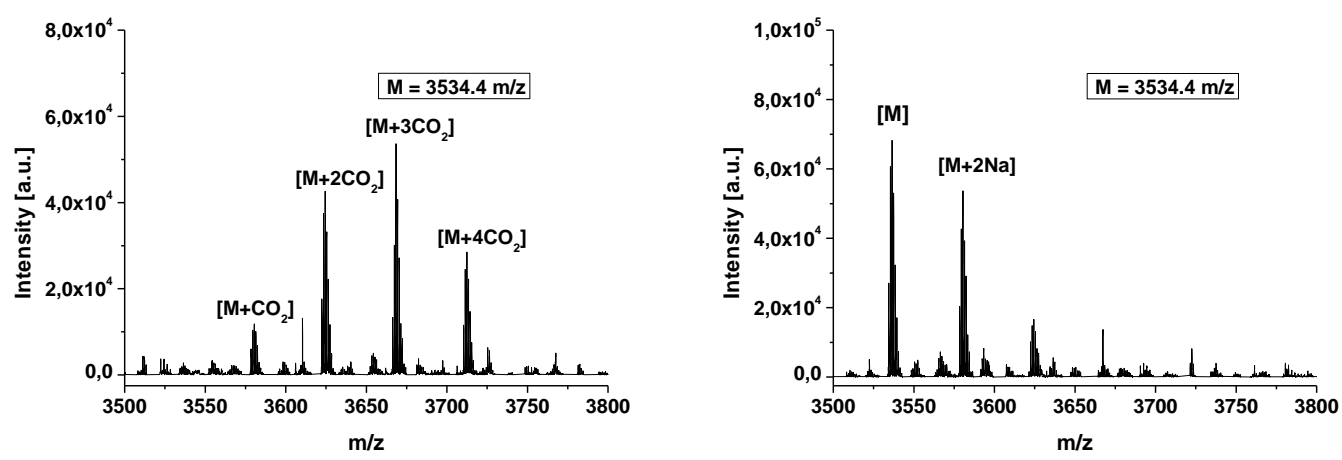

Figure 2.9 a) Reaction schema of the deprotection of $\beta^{3}$-hTrp with TFA. In a TFA solution, the side chain may be incompletely deprotected. By an acidic aqueous workup, the complete decarboxylation takes place. b) Deconvoluted ESI mass spectra of a peptide with incomplete Trp deprotection (left) and mass spectra of the same sample after lyophilisation with a mixture of dioxane and dilute $\mathrm{HCl}$ (right).

This leads to an increase of the measured mass by the number of $\beta^{3}-h \operatorname{Trp}(n \times 44 \mathrm{~m} / \mathrm{z})$. The excess of $\mathrm{CO}_{2}$ can be removed by lyophilisation with a mixture of dioxane or water with dilute $\mathrm{HCl}^{[103]}$

\subsubsection{Automatic microwave-supported peptide synthesis}

The development of automatic peptide synthesis is a relevant working method and is recently gaining importance. During the last decades, methods were developed to reduce expenditure of time and chemical consumption considerably in comparison to the standard manual method. ${ }^{[98,100,104-106]}$ The complex automatic peptide synthesis of $\beta$-peptides was among others, examined by GELLMAN et al. with the synthesis of an established 6-mer peptide that formed a 12-helix without any results. ${ }^{[9]}$ In contrast, with similar reaction conditions it was possible to synthesis several 9-mer peptides that formed 14-helices. This 
peptide synthesis was performed with $\mathrm{HBTU} / \mathrm{HOBt}$ at $\mathrm{RT}^{[53]}$ A much longer mixed 17 -mer $\left(16 \times \beta^{3}\right.$ - and $1 \times \beta^{2}$-homoamino acids) $\beta$-peptide was successfully synthesised by LELAIS from the SEEBACH group. ${ }^{[107]}$ However, most of the synthesis conditions for $\beta$-peptides show long reaction times and coupling steps at RT.

An increase in efficiency of $\alpha$-peptides synthesis was achieved by heating during the elongation step with conventional oil bath or microwave irradiation. ${ }^{[101,102]}$ The beneficial effect of the temperature increase was already successfully used for the manual synthesis of $\beta$-peptide (see Chapter 2.5.1). ${ }^{[98,100]}$ As a result of this, it could be advantageous to combine the established benefits of microwave synthesis with an automatic system for $\beta$-peptides. ${ }^{[38,94]}$ In order to develop a synthesis that is more time-efficient, two different syntheses have been developed. These methods differ in the choice of coupling reagents, the number of couplings and the coupling time. The first method (Method 1) is a combination of the established manual microwave-assisted SPPS (see Chapter 2.5.1) and a modified method based on the work of CAPORALE et al. ${ }^{[108]}$ CAPORALE et al. found an improvement of the synthesis of aggregating peptide sequences by using a double coupling strategy with DIC/Oxyma and HATU/Sym-collidine. Due to this work, the first coupling step was performed in Method 1 with DIC/Oxyma at $90{ }^{\circ} \mathrm{C}$ with a reaction time of $7.0 \mathrm{~min}$ (60 s preactivation at RT, $300 \mathrm{~s}$ ramp at $90{ }^{\circ} \mathrm{C}, 60 \mathrm{~s}$ cool down to RT) what constitutes an adapted method of the HE-SPPS method developed by CEM. ${ }^{[98]}$ The second coupling step of Method 1 was performed with HATU/HOAt/DIPEA at $75{ }^{\circ} \mathrm{C}$ with a reaction time of $20 \mathrm{~min}\left(60 \mathrm{~s}\right.$ preactivation at $\mathrm{RT}, 1140 \mathrm{~s}$ at $\left.75^{\circ} \mathrm{C}\right)$. The second method (Method 2) was based on the CarboMAX ${ }^{\mathrm{TM}}$ method by CEM. ${ }^{[104,105]}$ Here, the coupling takes place with DIC/Oxyma at $105^{\circ} \mathrm{C}$ for $2.6 \mathrm{~min}\left(155 \mathrm{~s} \mathrm{ramp}\right.$ at $\left.105^{\circ} \mathrm{C}\right)$. In contrast to the DIC/Oxyma coupling step used in Method 1, the CarboMAX ${ }^{\mathrm{TM}}$ method is based on the double amount of DIC in relation to the amino acid. In Table 2.3 an overview of the reaction conditions of the methods and in Appendix A the detailed settings, synthesis scale and quantities for the automatic peptide synthesis are given. Both methods were tested at a LibertyBlue ${ }^{\mathrm{TM}}$ peptide synthesiser. The uncoupled peptide was capped with $\mathrm{Ac}_{2} \mathrm{O}$ during the synthesis to increase the efficiency of the coupling, especially for longer peptide sequences. In addition, an easier purification of uncoupled subsequences is possible. For this, an adapted capping solution was used. This was developed by EMD MiLLIPORE (Burlington, Massachusetts, United States) and based on $\mathrm{Ac}_{2} \mathrm{O} / \mathrm{Oxyma}$ Pure/DIPEA. This mixture allows the use of a more diluted solution of acetic anhydride and shows better results 
compared with the standard capping solution (MERCK information brochure "Applications of Oxyma Pure and K-Oxyma Pure in Fmoc SPPS"). The deprotection of the $N$-terminal Fmoc group was performed with a standard deprotection solution of $20 \%$ piperidine in $\mathrm{DMF}$ at $90{ }^{\circ} \mathrm{C}$.

Table 2.3 Overview of the reaction conditions of the automatic microwave-supported SPPS for Method 1 and Method 2.

\begin{tabular}{|cccc|}
\hline & \multicolumn{2}{c|}{ Method 1 } & Method 2 \\
\hline Coupling reagents & DIC/Oxyma & HATU/HOAt/DIPEA & DIC/OXYMA \\
\hline Coupling time & & & \\
Preactivation & $60 \mathrm{~s}$ & $60 \mathrm{~s}$ & - \\
Microwave SPPS & $300 \mathrm{~s}$ & $1140 \mathrm{~s}$ & $155 \mathrm{~s}$ \\
\hline Coupling temperature & $90{ }^{\circ} \mathrm{C}$ & $75{ }^{\circ} \mathrm{C}$ & $105{ }^{\circ} \mathrm{C}$ \\
\hline
\end{tabular}

HPLC measurements and mass spectrometric examinations were carried out to determine the success of both methods. For this purpose, the peptide was cleaved from the resin, lyophilised, dissolved in HFIP and measured with HPLC. The main signals of the HPLC were collected and examined by ESI-MS.

To test the efficiency of the methods, two different peptides were used. Method 1 was used for a peptide shown in Figure 2.10 Pep Met 1, which was developed by KLOOS (unpublished results). The $C$-terminal amino acid D- $\beta^{3}$-hLys was protected on the side chain with Mtt. In contrast, the remaining D- $\beta^{3}$-hLys was protected with Boc at the side chain group.

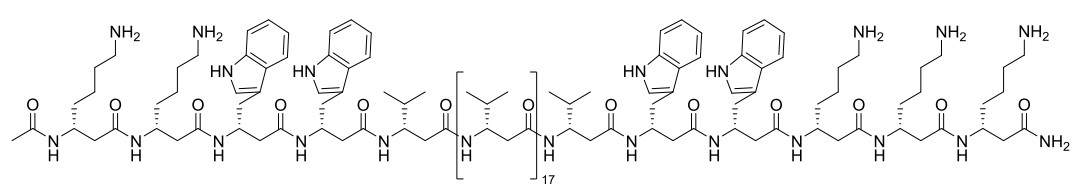

Pep Met 1 [3718.6 g/mol]

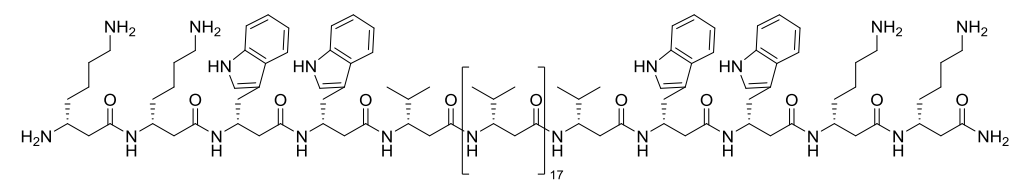

Pep Met 2 [3534.4 g/mol]

Figure 2.10 Structure of the used test peptides to determine the synthesis efficiency of automatic microwave-assisted SPPS.

Compared to Method 1, Method 2 works at higher temperatures and the thermal stability of the Mtt protecting group is unknown. Though, it is known that the highly acid-labile 
2-chlorotrityl resin, which has as linker a similar basic structure as Mtt, is instable at higher temperatures. This peptide Pep Met 2 shown in Figure 2.10, was used as $\beta$-peptide based transmembrane regions and was synthesised in the presented work using Method 2. ${ }^{[1]}$ The quality of the synthesised peptides was determined by HPLC. For this purpose, the crude peptide was lyophilised with dilute $\mathrm{HCl}$ solution and then dissolved in hexafluoro-2-propanol (HFIP). HARA et al. was able to show that the use of fluorinated alcohols, such as trifluoroethanol, facilitates the purification of hydrophobic and aggregation-prone transmembrane peptides. For this purpose, TFE was added up to $10-$ $16 \%$ to the mobile phase. ${ }^{[109]}$ In cooperation with SCHIRMACHER, it could be shown that a combination of solving the sample in HFIP and the addition of $0.1 \%$ HFIP into the mobile phase leads to an improved splitting of the peaks in the HPLC chromatogram (unpublished results). Figure 2.11 shows the HPLC chromatogram of Pep Met 1 and Figure 2.12 displays the MS analysis data of the main peaks. The HPLC chromatogram of the Pep Met 1 (see Figure 2.11) shows two main signals at $t_{\mathrm{R}}=14.7$ and $17.3 \mathrm{~min}$. MS analysis of the two peaks revealed in the peak at $t_{\mathrm{R}}=14.7 \mathrm{~min}$ the desired product with a mass of $3718.6 \mathrm{~mol} / \mathrm{g}$. The peak at $t_{\mathrm{R}}=17.3 \mathrm{~min}$ has a mass greater by $96 \mathrm{~m} / \mathrm{z}$ (see Figure 2.12).

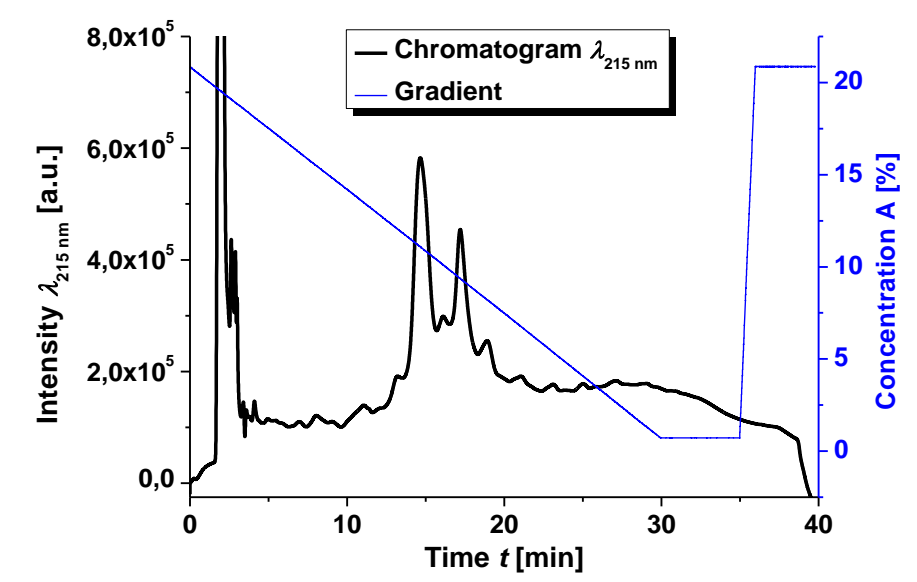

Figure 2.11 HPLC chromatogram of the crude peptide Pep Met 1. Absorption was recorded at 215 nm. Analytical HPLC was performed using a gradient $80 \rightarrow 100 \% \mathrm{~B}\left(\mathrm{~A}: \mathrm{H}_{2} \mathrm{O}+0.1 \%\right.$ TFA $+0.1 \%$ HFIP and B: $\mathrm{MeOH}+$ $0.1 \%$ TFA + $0.1 \%$ HFIP) in $30 \mathrm{~min}$, flow $1.0 \mathrm{~mL} / \mathrm{min}$ and $50{ }^{\circ} \mathrm{C}$. The sample was dissolved in $100 \%$ HFIP.

This mass difference can be explained, inter alia, by the addition of a TFA molecule, which is bound to one of the lysine side chains via an amide bond. RIzO et al. found that contamination in TFE can cause trifluoroacetylation at the lysine side chain. ${ }^{[110]}$ Whether this reaction is caused by impurities in the HFIP is unclear. Pep Met 2 was also treated under the same conditions with the same HFIP batch. Perhaps the residues of Mtt 
deprotection associated with HFIP leaded to a trifluoroacetylation. Another possibility is the selective deprotection of Mtt which was performed with a mixture of DCM/HFIP/TFE/TIS ( $v / v$ 65:20:10:5) at the resin-bound peptide. The Mtt deprotected resin-bound peptide was then split in two fractions. One part was used for further investigations by KLOOS and the other part was cleaved from the resin and analysed (Pep Met 2). However, these hypotheses require further research. All in all, it can be assumed that Method 1 leads to a successful automated SPPS.

a)

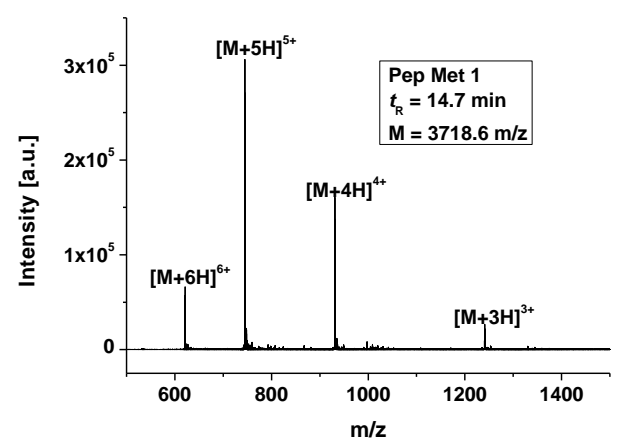

b)

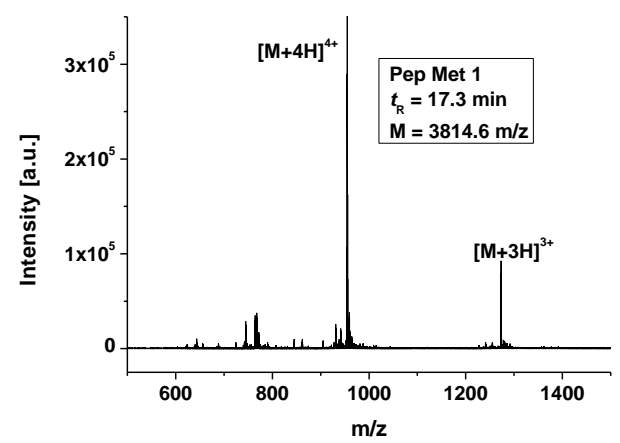

Figure 2.12 ESI mass spectra of Pep Met 1 at a) $t_{\mathrm{R}}=14.7 \mathrm{~min}$ and b) $t_{\mathrm{R}}=17.3 \mathrm{~min}$.

In the HPLC chromatogram of the Pep Met 2 (see Figure 2.13) is a signal increasing in the range from $12 \mathrm{~min}$ to $20 \mathrm{~min}$ with an maximum peak at $t_{\mathrm{R}}=15.8 \mathrm{~min}$. MS analysis of the this main peak showed the desired product with a Mass of $3534.4 \mathrm{~mol} / \mathrm{g}$ (see Figure 2.14 a).

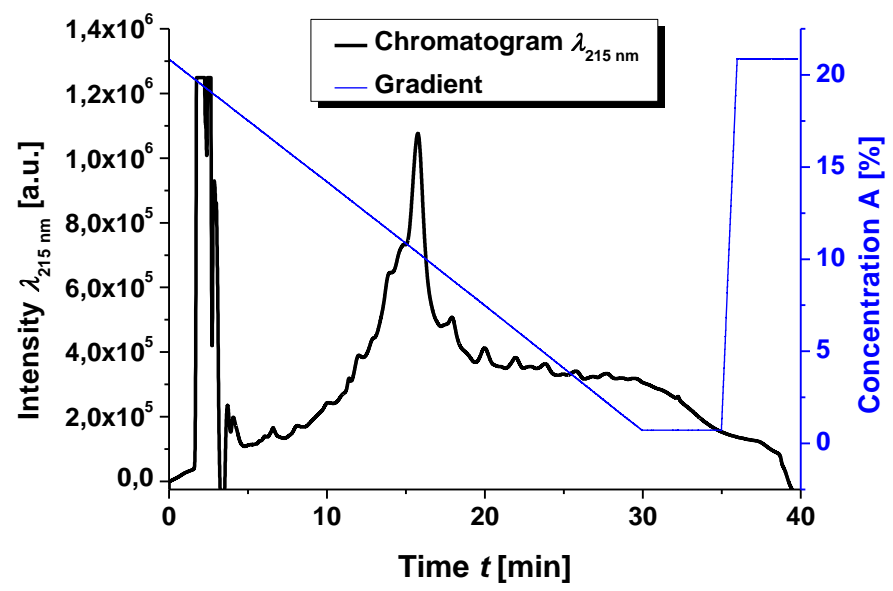

Figure 2.13 HPLC chromatogram of the crude peptide Pep Met 2. Absorption was recorded at 215 nm. Analytical HPLC was performed using a gradient $80 \rightarrow 100 \% \mathrm{~B}(\mathrm{~A}: \mathrm{H} 2 \mathrm{O}+0.1 \%$ TFA $+0.1 \%$ HFIP and B: $\mathrm{MeOH}+0.1 \%$ TFA + 0.1 \% HFIP) in $30 \mathrm{~min}$, flow $1.0 \mathrm{~mL} / \mathrm{min}$ and $50{ }^{\circ} \mathrm{C}$. The sample was dissolved in $100 \% \mathrm{HFIP}$.

The less resolved chromatogram of Pep Met 2 compared to Pep Met 1 can be explained by a higher concentration of raw product as well as the different structure. In comparison 
to Pep Met 1, Pep Met 2 has one D- $\beta^{3}$-hLys less. This lower number of charged amino acids is a main reason for the water solubility of the used peptides and leads to a poorer resolution of the chromatograms. ${ }^{[88,89]}$ In Figure $2.14 \mathrm{~b}$ ) the enlarged mass spectrum of Pep Met 2 in a range of $706-720 \mathrm{~m} / \mathrm{z}$ is given. In addition to the signal of the desired target peptide $[\mathrm{M}+5 \mathrm{H}]^{5+}$, two further signals with a mass difference of $14 \mathrm{~m} / \mathrm{z}$ respectively $28 \mathrm{~m} / \mathrm{z}$ are shown. These signals may be due to methylation of the target peptide on the $\mathrm{D}-\beta^{3}$-hLys side chains. Whether this side reaction occurs during peptide synthesis or through the MS analysis is still unclear. However, this side reaction requires further research. All in all, it can be assumed that Method 2 leads to a successful automated SPPS.

a)

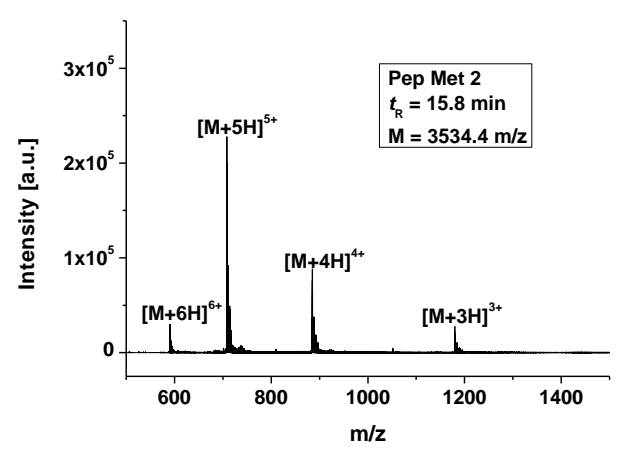

b)

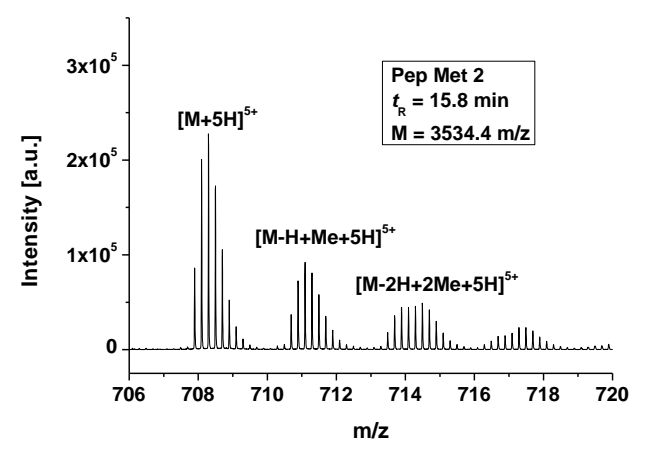

Figure 2.14 ESI mass spectra of Pep Met $2 t_{\mathrm{R}}=15.8 \mathrm{~min}$ from a) $500-1500 \mathrm{~m} / \mathrm{z}$ and from b) $706-720 \mathrm{~m} / \mathrm{z}$.

In summary, in the context of this work, two methods have been developed which make it possible to carry out an automatic SPPS of $\beta$-peptides. These methods allow the reduction of the above-mentioned peptides from $4-5 \mathrm{~d}$ to less than $1 \mathrm{~d}$. The analysis of both methods with HPLC chromatograms and subsequent MS analysis show the desired target peptide. However, both reactions showed peptides with undesired masses, which may be due to side reactions. These side reactions require further analysis to completely exclude a connection with the peptide synthesis or to adapt the automatic SPPS by e.g. the reduction of the coupling time. Furthermore, the isomerisation of the individual amino acids was not investigated and the degree of isomerisation per coupling is unclear. Nevertheless, methods have been introduced to significantly reduce the time spent on laboratory and to allow an efficient synthesis of $\beta$-peptides which can significantly accelerate the understanding of $\beta$-peptides. 


\section{Transmembrane $\beta$-peptides functionalised with fluorescence and IR-sensors}

Due to the hydrophobicity of transmembrane peptides and the membrane environment, both the synthesis and investigation are a difficult task. Sensor molecules and peptidomimetics are often used to clarify precise scientific research questions, like protein properties, protein localisation or protein interaction. ${ }^{[6,42]}$ In the following, transmembrane $\beta$-peptides, with two different secondary structures, which function as peptidomimetics are shown. Additionally, these transmembrane $\beta$-peptides have either indole or nitrile based sensors incorporated in their primary structures.

\subsection{Fluorescence-based sensors}

Biological systems are sensitive towards changes in their environment which might lead to biological responses. ${ }^{[1]}$ As an example, the transmembrane ATP synthase can transport protons across the membrane as well synthesis ATP by a proton gradient. To study these biological responses, different methods were developed such as spectroscopic-based methods like fluorescence or electron spin resonance spectroscopy. ${ }^{[111]}$ Especially in the last 30 years the biochemical research has benefited from the improvement of the fluorescent techniques. ${ }^{[112]}$ Fluorescence spectroscopy offers high sensitivity, fast response time, selectivity, experimental simplicity and flexibility. ${ }^{[12]}$ Fluorescence is the spontaneous emission of light by a substance that has absorbed light of an appropriate wavelength. ${ }^{[113]}$ Irradiation with light causes the electrons to be excited to an energetically higher state, from which they can follow different nonradiative or radiative relaxation processes to return to the ground state. Fluorescence is a radiative process, which means that energy is released by emitting photons. ${ }^{[113]}$ Since internal crossing to lower vibrational states usually precedes fluorescence, the emitted light has a higher wavelength than the light that was used for excitation, a phenomenon that is called STOKES shift. Phosphorescence is another radiative process which follows after intersystem crossing to a triplet state. The JABLONSKI diagram is a common method to illustrate the processes that may happen after excitation (see Figure 3.1). ${ }^{[12,114,115]}$

Labelling proteins with fluorescent molecules is a common method to study biological processes. ${ }^{[112,116-119]}$ For example fluorescence-labelled antibodies or cell proteins reveal the localisation in the cell by fluorescence microscopy is used. ${ }^{[1]}$ However, the synthesis of these proteins is often tedious because of their complex structure. ${ }^{[118,120]}$ Here, peptides represent a useful alternative, as they have been widely studied over the past years. ${ }^{[121]}$ Due 
to the fact that they exhibit a less complex structure they are easier to synthesise which allows fast and easy modifications of the structure. ${ }^{[98,122]}$ Compared to small molecules, peptides feature similar structures as proteins and thus allow mimicking functional areas or binding positions in the native protein. ${ }^{[12,119]}$

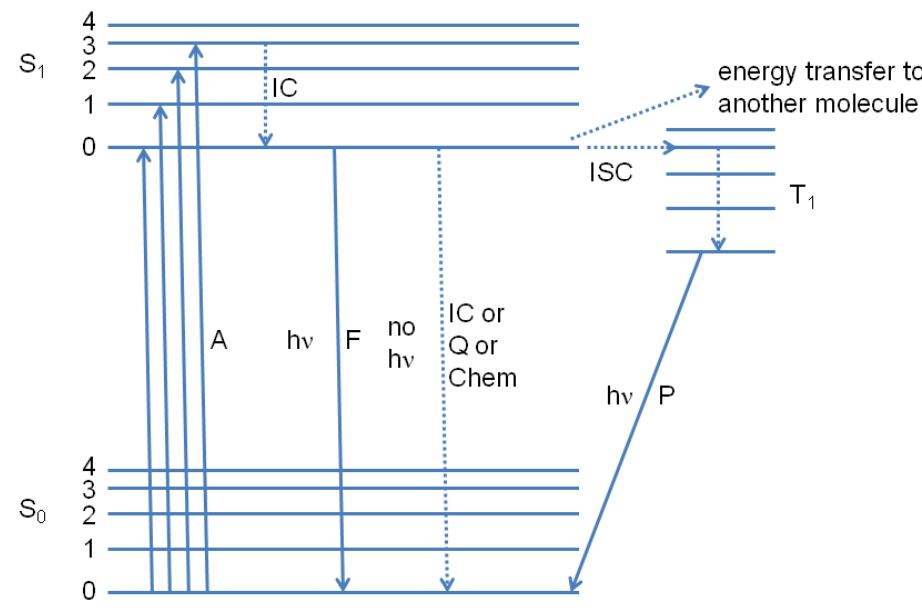

Figure 3.1 JABLONSKI diagram with absorption (A), internal conversion (IC), intersystem crossing (ISC), fluorescence $(F)$, light emission $(h v)$, thermal deactivation $(Q)$, chemical reaction $(C h e m)$ and phosphorescence (P). ${ }^{[112,114,115]}$

In addition to fluorescence-based markers, fluorophores with controllable fluorescent properties enable the use as fluorescence-based sensors and can be classified into three groups. The first group comprises sensors that are environment-sensitive and change their fluorescence for example upon increasing the hydrophobicity. The second group consists of sensors that react to concentration changes of anions, cations or neutral molecules. The third group of sensors allows studying the location or movement of biomolecules by undergoing "through-the-space" effects like FRET. ${ }^{[12]}$ A fluorescence change caused by the environment is mostly based on a large excited-state dipole moment of the fluorophore. Due to this, the fluorophore usually shows a low quantum yield in an aqueous solution, resulting in a weak fluorescence emission. In organic solvents or hydrophobic surroundings a high fluorescence emission is expected, which is blue-shifted. ${ }^{[12]}$ Some well-known examples for fluorescence-based sensors are NBD, Dansyl and the natural aromatic amino acids. The change of fluorescence caused by the coordination of an ion is usually based on a chelation-enhanced fluorescence mechanism, which involves a change in the electronic structure of the system by the addition of an ion (see Chapter 4.2). A variety of fluorescence-based sensors has been developed for a multitude of ions and molecules. ${ }^{[123-126]}$ Through-the-space effects are based on interactions of one or more types of fluorophores, which lead to quenching by intermolecular energy transfer. ${ }^{[38,89,127,128]}$ 
In order to study peptides and proteins by fluorescence spectroscopy, a fluorophore is necessary. Beside unnatural fluorophores, natural amino acids can be exploited for fluorescence spectroscopic measurements. ${ }^{[12,116-119]}$ For this, the aromatic amino acids tryptophan, tyrosine and phenylalanine can be used. ${ }^{[129]}$ The most common fluorescent-based sensor is tryptophan as it is present in a lot of proteins and biological active peptides. ${ }^{[129]}$

\subsection{Design of tryptophan incorporated model $\beta$-peptides}

The complexity of the most natural transmembrane helical peptides made it necessary to develop model systems which can be easily modified and characterised. For the natural $\alpha$-peptides a broad spectrum of $\alpha$-model peptides were developed and tested for their properties. ${ }^{[7,128,130-132]}$ All of these peptides have a hydrophobic core which can be flanked by more hydrophilic amino acids. Based on this $\alpha$-model peptides, $\operatorname{RosT}^{[11]}$ and PETERSEN, ${ }^{[12]}$ developed transmembrane $\beta$-model peptides. The synthesised $\beta$-peptides in the following chapter were adapted and modified from PETERSEN ${ }^{[12]}$ and inspired by KALP and $\mathrm{L}_{24}$ model peptides. ${ }^{[7]}$

The design of the following $\beta$-model peptides is based on a hydrophobic core which is flanked on each side by two $\beta^{3}$-homolysines $\left(\beta^{3}\right.$-hLys). Lysine is known to increase the solubility of the peptides and it supports anchoring of these peptides in lipid membranes. ${ }^{[88,89]} \beta^{3}$-Homotryptophan $\left(\beta^{3}\right.$-hTrp) was used as environment sensor and placed central or close to terminal position of the hydrophobic core. Tryptophan is built up from an $\alpha$-amino group, $\alpha$-carboxylic acid group as well as an indole as side chain functionality and it shows characteristic fluorescence behaviour due to the large aromatic indole system. ${ }^{[133,134]}$ Additionally, the uncharged indole ring has the highest hydrophobicity of the 20 different amino acids side chain groups. ${ }^{[135]}$ Together with the polar NH group, tryptophan generates an amphiphilic character which allows to form hydrogen bonds. ${ }^{[136]}$ This amphiphilic character leads to the good anchoring properties of the amino acid tryptophan in lipid bilayers. However, the fluorescence properties of the tryptophan are sensitive to the environment. This effect can be used to analyse the hydrophobicity of the surrounding of peptide-bound tryptophan. ${ }^{[11,16-20]}$ It has been shown that tryptophan in a hydrophobic surrounding, like the inner membrane part, has a fluorescence maximum of $\lambda_{\max }<330 \mathrm{~nm} .^{[16-19]}$ In a more hydrophilic part, like the headgroup region, tryptophan shows a red-shift with a fluorescence maximum of 
$\lambda_{\max }>330 \mathrm{~nm} \cdot{ }^{[18,20]}$ Tryptophan in aqueous solution shows a fluorescence emission maximum of approximately $350 \mathrm{~nm} .^{\text {[21] }}$

The length of the hydrophobic core was adjusted according to span the entire lipid bilayer. As calculation basis, 3.0 amino acids per turn (1.56 $\AA$ rise/residue) were considered for the 14-helix and 2.5 residues per turn $(2.1 \AA$ rise/residue) for the 12-helix (see chapter 2.1$) .{ }^{[10]}$ Based on the different structure properties of the helices, the number of amino acids in the hydrophobic core of the 12-helix formed peptide was modified, unlike the work of PETERSEN. ${ }^{[12]}$ The length of the hydrophobic region $\left(2 \mathrm{D}_{\mathrm{c}}\right)$ in the lipid bilayer varies depending on the length of the acyl chains, the lipid composition of the membrane and the lipid phase (see Table 3.1). The used model membranes were built by one species of phosphatidylcholine lipids, which varied in the length and saturation grade. The following lipids with the corresponding hydrophobic region length were provided: 1,2-dimyristoylsn-glycero-3-phosphocholine (DMPC， $19-26 \AA),{ }^{[137-141]}$ 1,2-dipalmitoyl-sn-glycero-3phosphocholine (DPPC, 26-29 $\AA)^{[137,139,140]}$ and 1,2-dioleoyl-sn-glycero-3-phosphocholine (DOPC, $27-30 \AA$ ) ${ }^{[137,139,142]}$

Table 3.1 Length of the hydrophobic core of the liposome $\left(2 D_{c}\right)$.

\begin{tabular}{|cccc|}
\hline & DMPC $^{[137-140]}$ & DPPC $^{[137,139,140]}$ & DOPC $^{[137,139,140]}$ \\
\hline 2D $_{\mathbf{c}}(\mathbf{A})$ & $19.0-26.2$ & $26.0-28.5$ & $27.0-30.0$ \\
\hline
\end{tabular}

The large variance in the listed values is due to the complexity of lipid systems. In a monolipid system, environmental factors, such as the degree of hydration or the ambient temperature play a significant role and influence, inter alia, the lipid phase ${ }^{[139]}$ Based on these data, the hydrophobic sequence of the right-handed 14-helices was assembled with $19 \beta^{3}$-amino acids, which leads to a length of about $30 \AA$. For the thinner and stretched 12-helical $\beta^{3}$-peptide, 15 amino acids were placed in the hydrophobic core which leads to a length of about $31 \AA$ (see Table 3.2).

Table 3.2 Length of the hydrophobic peptide region of the different used helices based on the number of hydrophobic amino acids (AA).

\begin{tabular}{|cc|}
\hline 12-helix & 14-helix \\
\hline $31.5 \AA(15 \mathrm{AA})$ & $29.6 \AA(19 \mathrm{AA})$ \\
\hline
\end{tabular}

Based on these considerations ten different peptides were synthesised (see Figure $3.2 \&$ Figure 3.3). However, to receive the desired secondary structures, the primer sequences of the hydrophobic core have to be considered. To obtain a right-handed 14-helices based on the branch of the used amino acids D- $\beta^{3}$-homovaline $\left(D-\beta^{3}-h\right.$ Val $)$ and D- $\beta^{3}$-homoisoleucine 
(D- $\beta^{3}$-hIle) were used. A left-handed 12-helix can be induced by $D-\beta^{3}$-homoleucine $\left(D-\beta^{3}\right.$-hLeu) (see Figure 3.2). ${ }^{[75,83]}$

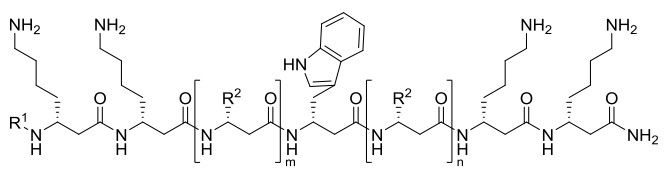

\begin{tabular}{|ccccc|}
\hline & $\mathbf{R}^{1}$ & $\mathbf{R}^{2}$ & m & $\mathbf{n}$ \\
\hline Pep 1 & H & Val & 9 & 9 \\
\hline Pep 2 & H & Val & 1 & 17 \\
\hline Pep 3 & H & Ile & 9 & 9 \\
\hline Pep 4 & H & Ile & 1 & 17 \\
\hline Pep 5 & H & Leu & 7 & 7 \\
\hline Pep 6 & H & Leu & 1 & 13 \\
\hline Pep 7 & Ac & Leu & 7 & 7 \\
\hline Pep 8 & Ac & Leu & 1 & 13 \\
\hline
\end{tabular}

Figure 3.2 Schematic structure of the designed $\beta^{3}$-peptides Pep 1 - Pep 8 for checking the influence of branching on the secondary structure of transmembrane $\beta^{3}$-peptides.

The possibility to force a left-handed transmembrane 12-helix into a right-handed transmembrane 14-helix was examined by the steric ring structure $(1 R, 2 R)$-trans-2aminocyclohexane carboxylic acid (ACHC). ${ }^{[10,74,83]}$ Therefore, a peptide sequence based on $\mathrm{D}-\beta^{3}$-hLeu was chosen, which is interrupted at every third amino acid with ACHC (see Figure 3.3).

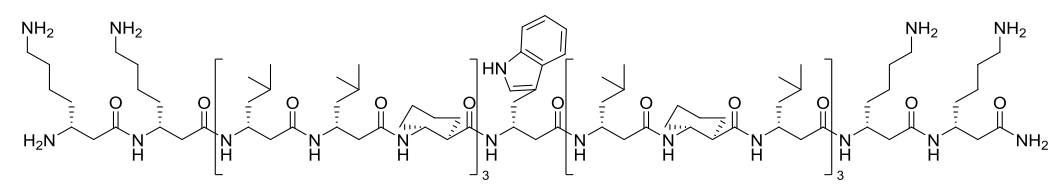

Pep 9

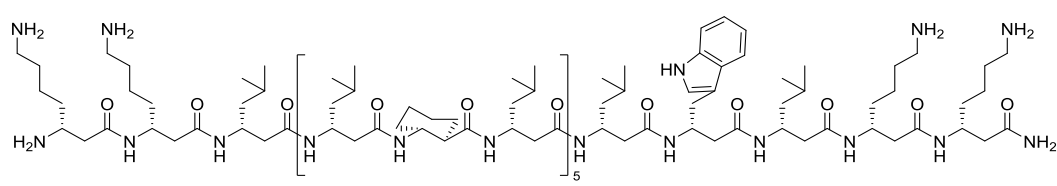

\section{Pep 10}

Figure 3.3 Chemical structure of the synthesised $\beta$-peptides Pep 9 and Pep 10.

The carboxylic acid of the $C$-terminus was transferred in a carboxylic acid amide during the peptide synthesis. Studies showed that carboxyl groups like aspartic acid or glutamic acid influence the incorporation of transmembrane peptides and the $\mathrm{pH}$ stability. ${ }^{[143]}$ The influence of a free $\mathrm{N}$-terminus was tested for left-handed 12-helices formed peptides. Therefore, the $N$-terminus was free and able to build up a positive charge or it was eliminated by acetylation with acetic anhydride what resulted in an $\mathrm{N}$-terminal amide bond. The acetylated right-handed 14-helices were synthesised and characterised by 
PETERSEN. ${ }^{[12]}$ In particular, the $\beta^{3}-\mathrm{hVal}$ containing peptides showed stable formation of a secondary structure in organic solvents and model lipid systems. These results are in contrast to PETERSEN hypotheses of the destabilisation of the peptide structure by $N$-terminal modification. ${ }^{[12]}$ However, the results obtained are supported by studies of Seebach et al. which were unable to detect a significant difference from $N$-terminal protected $\beta$-peptides to $N$-terminal unprotected $\beta$-peptides. ${ }^{[144]}$ The synthesis of the peptides was carried out in accordance with the manual SPPS of $\beta$-peptides. ${ }^{[11-13,15,145]}$ As indicated in previous studies, the synthesis of 14-helix formed repetitive peptide sequence of D- $\beta^{3}$-hVal or D- $\beta^{3}$-hIle (Pep 1 - Pep 4), showed a high efficiency. Because of this, a single coupling step was sufficient for these hydrophobic amino acids. ${ }^{[1-13]}$ The 12-helix formed peptides (Pep 5-Pep 8), consisting predominantly of hydrophobic D- $\beta^{3}$-hLeu showed less coupling success, what is known from other studies. ${ }^{[12,13]}$ In contrast to ZANBOT, ${ }^{[13]}$ this study shows that it was possible to obtain a sufficient amount of the full-length peptide by triple coupling, with 5.00 eq amino acid for each coupling step. These synthesis problems did not appear by the ACHC containing leucine peptides (Pep 9-Pep 10). This may be due to interactions during the synthesis with the resin, the growing peptides or a poorer accessibility of the 12-helix $N$-terminus during the synthesis. In all peptides (Pep 1 - Pep 10) tryptophan was double coupled. The same procedure was done in Pep 9 - Pep 10 regarding the coupling of ACHC. This was done in order to obtain a higher yield for these coupling steps.

The formed secondary structure and the verification of the incorporation in phospholipid membranes are in focus of the following analysis. To confirm the structure of the peptides, the results of recorded CD spectroscopy were compared with literature known spectra. The incorporation was verified by the maximum tryptophan fluorescence $\left(\lambda_{\max },\right)$. Therefore, the peptides were measured in organic solvents like $\mathrm{MeOH}$ and TFE as well as in DMPC, DPPC, DOPC. The use of different lipid ensured the structure analysis by decreasing the negative mismatch.

\subsubsection{Measurements and results}

\section{Conformation studies}

The secondary structure of the synthesised $\beta$-peptides Pep 1 - Pep 10 was analysed by CD spectroscopy and compared with the literature. ${ }^{[62,64,70,74,75,77,78,83,146-150]}$ The CD spectra were measured in organic solvents $(\mathrm{MeOH}, \mathrm{TFE})$ and in LUVs with different lipid composition (DMPC, DPPC, DOPC) varying in the length of the hydrophobic 
core. ${ }^{[137,151,152]}$ From different peptides, like Gramicidin A or 14-helical $\beta^{3}$-peptides, it is known that TFE can stabilise the secondary structure. ${ }^{[81,153,154]}$

The CD spectra of Pep 1 in TFE $\left(\sim 50 \mu \mathrm{M}, \mathrm{T}=25^{\circ} \mathrm{C}\right)$ and in model lipid vesicle DMPC (LUVs, P/L-ratio $=6: 100, T=30^{\circ} \mathrm{C}$ ) are shown in Figure 3.4 a) with a minimum between $185 \mathrm{~nm}$ and $195 \mathrm{~nm}$ and a maximum between $200 \mathrm{~nm}$ and $220 \mathrm{~nm}$. The CD spectra of Pep 5 in TFE $\left(\sim 50 \mu \mathrm{M}, T=25^{\circ} \mathrm{C}\right)$ and in model lipid vesicle DMPC (LUVs, $\mathrm{P} / \mathrm{L}$-ratio $=5: 100, T=30^{\circ} \mathrm{C}$ ) are presented in Figure $3.4 \mathrm{~b}$ ) with a minimum between $190 \mathrm{~nm}$ and $200 \mathrm{~nm}$, a maximum between $200 \mathrm{~nm}$ and $210 \mathrm{~nm}$ and a second minimum between $210 \mathrm{~nm}$ and $230 \mathrm{~nm}$. The CD spectra of the $\beta$-peptides in organic solvent as well as in lipid model systems showed a similar and characteristic pattern. This indicates a 14-helix (Pep 1 - Pep 4, Pep 9-Pep 10) and a 12-helix (Pep 5-Pep 8), respecttively. ${ }^{[62,64,70,78]}$

a)

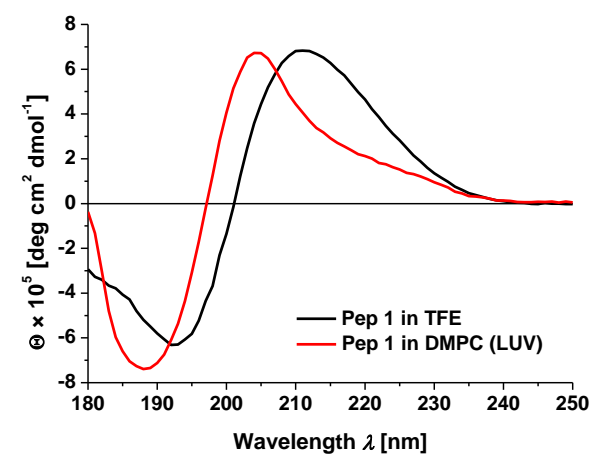

b)

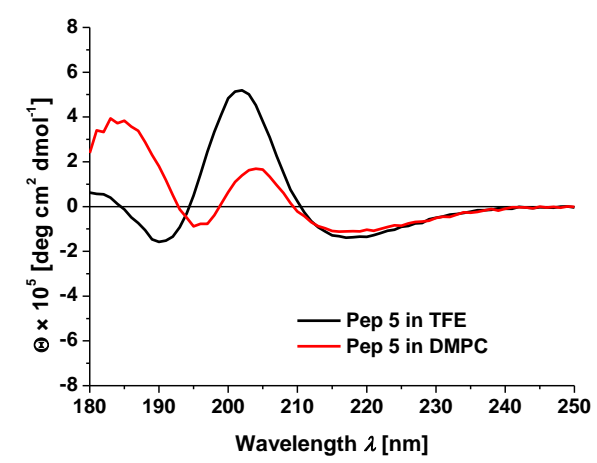

Figure 3.4 CD spectra of Pep 1 (a) and Pep 5 (b) in TFE (black, $\sim 50 \mu \mathrm{M}, T=25{ }^{\circ} \mathrm{C}$ ) and incorporated in DMPC LUVs (red, Pep 1: P/L ratio $=6: 100$, Pep 5: $\mathrm{P} / \mathrm{L}$ ratio $=5: 100, T=30{ }^{\circ} \mathrm{C}$ ).

The maxima and minima of the CD spectra in organic solvent (TFE, MeOH) of the Pep 1 Pep 4 and Pep 9- Pep 10 are slightly shifted compared to those spectra in lipid systems (DMPC, DPPC, DOPC). On the one hand, it is known from $\alpha$-peptides that a different dielectric constants of the surrounding solvents or lipids might be a reason for a shift in a CD spectrum. ${ }^{[155]}$ On the other hand, there may be structural changes of the peptide that result in a changed spectrum. The shift of the CD spectra between organic solvent and lipid membrane is more intensive in case of the 14-helix, compared with the 12-helix. Moreover, the blue shift and slight change in shape shown here is in accordance with a study of Pomerantz et al. ${ }^{[76]}$ PoMERANTZ et al. was able to show that the aggregation of 14-helix peptides leads to a change in the CD spectra by self-assembled 14-helix $\beta$-peptides. ${ }^{[76]}$ The amount of aggregated peptides increases with a higher content of buffer 
in a $\mathrm{MeOH} / \mathrm{buffer}$ solvent. The received $\mathrm{CD}$ spectrum from POMERANTZ et al. showed matches with the presented CD spectrums of Pep 1 - Pep 4 and Pep 9 - Pep 10 (see Figure $3.5)$.

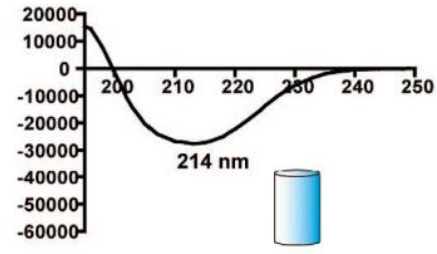

Peptide dissolved in $\mathrm{MeOH}$

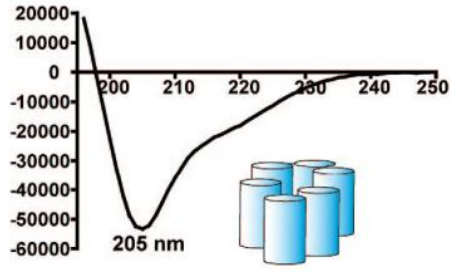

Peptide dissolved in buffer

Figure 3.5 The influence of aggregation on the CD spectrum of $\beta$-peptides in studies of PoMERANTZ et al. depending on the solvent composition. The aggregation causes a shift in the maximum from $214 \mathrm{~nm}$ to $205 \mathrm{~nm}$ and a sharpening of the signal. The signals are inverted unlike the ones used here, because of the use of L-amino acids. ${ }^{[76]}$ Reprinted with permission from W. C. Pomerantz et al., Org. Lett. 2008, 10, 1799-1802. Copyright (2008) American Chemical Society.

Based on these results, another reason for the shift in the CD spectra could be an aggregation in the lipid membrane because of lipid-peptide interaction. These results are attributable to the high concentration of the peptides (P/L-ratio 5:100). FöRSTER resonance energy transfer (FRET) studies of ROST et al. have shown that on a similar model system no unwanted aggregation at low peptide concentrations take place. ${ }^{[94]}$ The CD spectra intensity change and the shift of the maxima/minima of the 12-helix formed peptides Pep 5 - Pep 8 might be others beside the reasons mentioned above (see Figure 3.4). LEPLAE et al. and PARK et al. found that a 12-helix formed by $\beta^{3}$-peptide and $\beta^{2} / \beta^{3}$-peptide with cyclic amino acids (ACPC, ACP) shows a change in the $\mathrm{CD}$ spectra from $\mathrm{MeOH}$ to water. ${ }^{[75,149]}$ In $\mathrm{MeOH}$ the peptides delivered stronger $\mathrm{CD}$ signals than in water and the spectra showed a blue shift from methanol to water (see Figure 3.6).

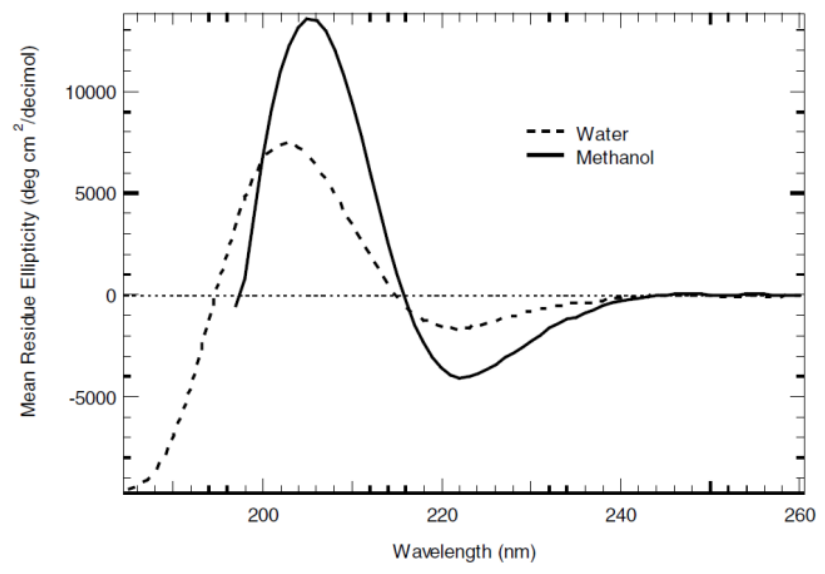

Figure 3.6 CD spectrum of 12-helix formed $\beta^{3}$-peptide with cyclic amino acids in MeOH and water. ${ }^{[75]}$ Reprinted with permission from P. R. LePlae et al., J. Am. Chem. Soc. 2002, 124, 6820-6821. Copyright (2002) American Chemical Society. 
Therefore, LEPLAE et al. and PARK et al. supposed a destabilising effect of water on the secondary structure of the used peptides. ${ }^{[75,149]}$ There are similarities by comparing these spectra with the peptide spectra of Pep 5-Pep 8. However, the observed spectra of peptide Pep 5 - Pep 8 showed no blue shift like the spectra in Figure 3.6, but a slight red shift. This red shift could be explained by a destabilisation of the secondary structure in the lipid system, but further investigations are needed to determine the exact structure in the membrane.

Based on the properties of ACHC it was possible to force the transmembrane structure formation from a 12-helix (Pep 5 - Pep 6) into a 14-helix with 19 amino acids (Pep 9 Pep 10). Hamuro et al. described that the intensity of the $\mathrm{CD}$ spectra depends on the length of the $\beta$-peptide in micelles of dodecylphosphocholine (DPC). With increased length of the $\beta$-peptides the $\mathrm{CD}$ intensity increases, which is in good agreement with the here presented results. ${ }^{[156]}$ These results clearly demonstrate that the synthesised $\beta$-peptides form stable secondary structures in solution and in lipid environment (see Appendix C).

\section{Membrane location studies}

In order to analyse the position of $\beta$-peptides in lipid membranes, the fluorescence property of tryptophan was used. This characteristic is known to be sensitive to the polarity of its local environment. The fluorescence maximum $\lambda_{\max }$ is shifted as soon as the surrounding of the peptide becomes more hydrophobic or hydrophilic. ${ }^{[16-19]}$ Previous studies have shown that in a hydrophobic surrounding such as the inner membrane part, tryptophan has a fluorescence maximum at $\lambda_{\max }<330 \mathrm{~nm}$. In a more polar environment, the fluorescence maximum shows a red-shift $\left(\lambda_{\max }>330 \mathrm{~nm}\right) .{ }^{[16-19]}$ This behaviour is based on a different dielectric constant of the nonpolar acyl chains and the polar headgroup. Polar groups induce an electron density shift in the indole-ring, which results in a red-shift of the fluorescence maximum. ${ }^{[18,157]}$ Figure 3.7 presents the fluorescence emission spectra of four $\beta$-peptides with $D-\beta^{3}$-hTrp located in different position and helix conformation (14-helix = Pep 1, Pep 2 Figure 3.7 a), 12-helix = Pep 7, Pep 8 Figure 3.7 b). The peptides with a bilayer midplane located D- $\beta^{3}$-hTrp (Pep 1, Pep 7), reveal a maximum at $\lambda_{\max , \text { Pep } 1}=318 \mathrm{~nm}$ in DOPC (LUVs, P/L-ratio 6:100, $T=25^{\circ} \mathrm{C}$ ) and at $\lambda_{\max , \text { Pep } 7}=318 \mathrm{~nm}$ in DMPC (LUVs, P/L-ratio 5:100, T=30 ${ }^{\circ} \mathrm{C}$ ). The fluorescence emission spectra of peptides with D- $\beta^{3}$-tryptophan near the apolar/polar interface (Pep 2, Pep 8) show a $\lambda_{\max , \mathbf{P e p} 2}=334 \mathrm{~nm}$ in DOPC (LUVs, P/L-ratio 6:100, $T=25^{\circ} \mathrm{C}$ ) and $\lambda_{\max , \mathbf{P e p}} 8=330 \mathrm{~nm}$ in DMPC (LUVs, P/L-ratio 5:100, $T=30^{\circ} \mathrm{C}$ ) (see Figure 3.7 and Appendix C). 
a)

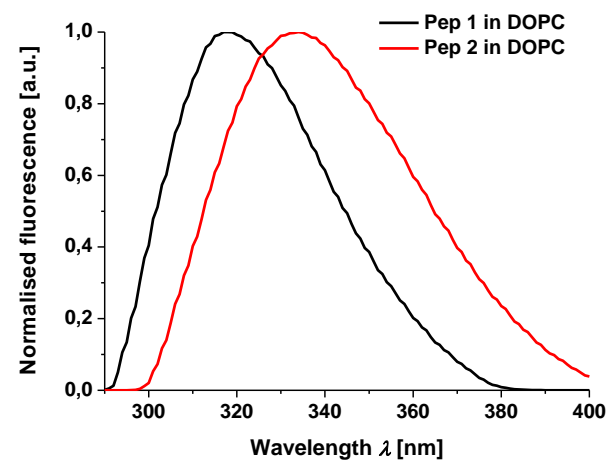

b)

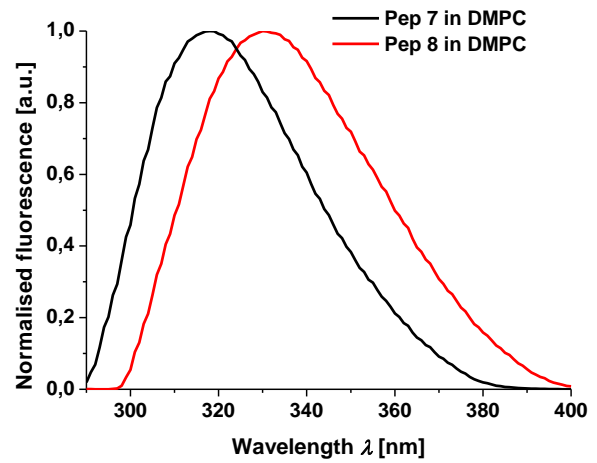

Figure 3.7 Fluorescence spectra of a) the 14-helix formed Pep 1 (black) and Pep 2 (red) incorporated in DOPC (LUVs, P/L-ratio 6:100, $T=25^{\circ} \mathrm{C}$ ) and of b) the 12-helix formed Pep 7 (black) and Pep 8 (red) incorporated in DMPC (LUVs, P/L-ratio 5:100, $T=30^{\circ} \mathrm{C}$ ).

However, due to the results of the fluorescence emission spectra of the $\beta$-peptides Pep 2 and Pep 8 it could be assumed that the D- $\beta^{3}$-hTrp is located at the apolar/polar interface, as it was expected. The fluorescence spectra of the $\beta$-peptides in Figure 3.7 with the central tryptophan (black line) show a blue-shift compared to the spectra of the $\beta$-peptides with the peripheral $D-\beta^{3}$-tryptophan (red line), which leads to the conclusion that these kinds of $\beta$-peptides penetrate the lipid bilayer and are not localised on the surface of the lipid bilayer. The fluorescence emission maxima of all analysed $\beta$-peptides in different membrane environments are given in Appendix C. Except for the not acetylated 12-helix formed peptides, Pep 5 and Pep 6, the fluorescence maxima show a blue-shift of the maxima from a hydrophobic to a more hydrophilic environment. Pep 5 and Pep 6 show artefacts in the fluorescence spectra, which disappear by the comparable acetylated peptides. In Figure 3.8 the fluorescence spectra of Pep 5 and Pep 7 are given. Pep 5 shows a narrow peak with a shoulder in organic solvent (TFE) and a broad peak in lipid surrounding (see Figure 3.8 a). Compared to Pep 5 the spectrum of Pep 7 in organic and lipid surrounding displays an expected spectrum with a broad curve and a clear maximum (see Figure $3.8 \mathrm{~b}$ ). The CD spectra of both peptide types (non-acetylated and acetylated) provide signals of approximately equal magnitude for organic solvents $\left(\Theta \sim 5 \times 10^{-5}\right.$ $\left.1.0 \times 10^{-6} \mathrm{deg} \mathrm{cm}^{2} \mathrm{dmol}^{-1}\right)$ and lipid systems $\left(\Theta \sim 0.5 \times 10^{-5}-2.0 \times 10^{-5} \mathrm{deg} \mathrm{cm}^{2} \mathrm{dmol}^{-1}\right)$. In general, PETERSEN assumed an effect of $N$-terminal acetylation on the peptide structure and properties of transmembrane $\beta$-peptides. ${ }^{[12]}$ In comparison with their work, the acetylation of the $\mathrm{N}$-terminus has apparently a stronger effect on the 12-helix than on the 14-helix. In order to be able to assess these results more precisely, further investigations are needed. One approach could be to determine the structural change from organic solvent 
to the lipid model system. Known methods for this would be for example, NMR-based structural analyses, fluorescence-based examinations or mesophase crystallisation. ${ }^{[20,158-160]}$

a)

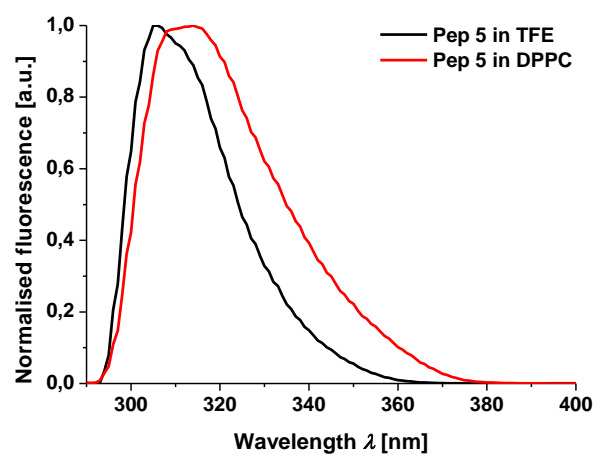

b)

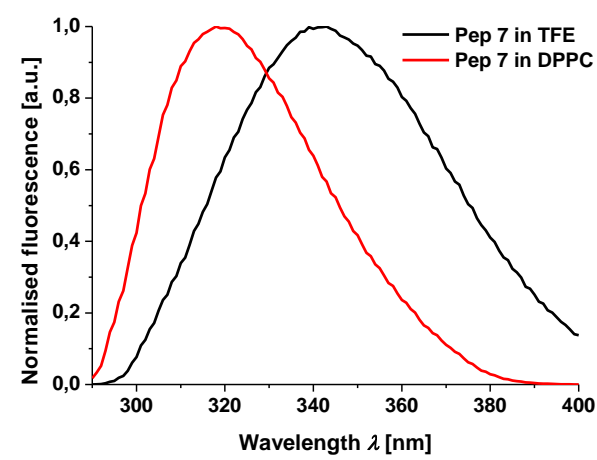

Figure 3.8 Fluorescence spectra of a) Pep 5 and b) Pep 7 in TFE(black line) as well as incorporated in DPPC (red line) $\left(\mathrm{LUVs}, \mathrm{P} / \mathrm{L}\right.$-ratio 5:100, $\left.T=50^{\circ} \mathrm{C}\right)$.

In addition to the structural information of the individual peptides, intermolecular interactions can also be investigated by the mentioned methods. ${ }^{[161,162]}$ This can help to improve the understanding of the measurement data.

\section{Conclusion}

The synthesis and characterisation of $\beta$-peptides constitute a wide and substantial topic in biochemistry. ${ }^{[9,44]}$ In the present study, new model systems for transmembrane $\beta$-peptides were designed, synthesised, purified and analysed. CD and fluorescent spectroscopic measurements were used to characterise the synthesised $\beta$-peptides Pep 1 - 10. The results prove that the secondary structures of $\beta$-peptides (12- or 14-helix) in organic solvent (MeOH, TFE) as well as in LUVs (DMPC, DPPC, DOPC) depend on the choice of side chains and can be controlled by ACHC. Most of the used $\beta$-peptides showed the presumed behaviour in LUVs. This behaviour was analysed by fluorescence spectroscopy of peripheral and central positioned tryptophan residues in the $\beta$-peptide sequences. These and previous findings on similar $\beta$-peptides described by RosT et al. indicate that the designed $\beta$-peptides are incorporated in lipid membranes in a transmembrane manner. ${ }^{[38]}$

The presented results demonstrate that the synthesised transmembrane $\beta$-peptides can be used in a wide research field. Other studies have shown that these kind of $\beta$-peptides are suitable for transmembrane $\beta$-peptide aggregation using $\mathrm{D}-\beta^{3}$-homoglutamine as a recognition unit. ${ }^{[94]}$ Hence, the introduced $\beta$-peptides are an appropriate model system for further experiments on transmembrane $\beta$-peptides. 


\subsubsection{Distance measurements by fluorescence quenching}

The results of the fluorescence measurements (see Appendix C) confirmed the incorporation of the synthesised $\beta$-peptides in the model membranes. To receive more detailed information, further methods have to be performed. Based on the tryptophan fluorescence and the interaction with other molecules the penetration depth of tryptophan can be calculated. ${ }^{[22-25]}$

The basis for the penetration depth of tryptophan is the fluorescence interference by quenchers. Quencher molecules do not influence the absorption behaviour of a fluorophore, though the quantum yield will be affected. ${ }^{[114,115]}$ The modified fluorescence behaviour is induced by a spin-orbit interaction what results in a $\left(\mathrm{S}_{1} \rightarrow \mathrm{T}_{1}\right)$-crossing (intersystem crossing, ISC, see Figure 3.1). This transition is reflected by a nonradiative transfer of energy from the fluorophore to a quencher molecule. ${ }^{[24,114,115,163]}$ For this transfer it is necessary that the molecules are located close to each other. Depending on the type of quenching mechanism it can be distinguished between static and dynamic fluorescence quenching. ${ }^{[24,114,115,163]}$ In the following, a static fluorescence quenching for the transmembrane peptide-bounded tryptophan mechanism is postulated. ${ }^{[20]}$ In the static fluorescence quenching mechanism the fluorophore and the quencher form a complex [FQ] which absorbs the light radiation $(h v)$ like a quencher free fluorophore. The excited state $[\mathrm{FQ}]^{*}$ relaxes in contrast to the quencher free fluorophore without fluorescence but through the emission of heat $(\Delta)$. Based on this concept by increasing the amount of quencher, the number of available fluorophore will be decreased. In Equation 3.1, the processes for a quencher free process is given, whereas in Equation 3.2 the process for the static fluorescence quenching mechanism is displayed. ${ }^{[24,114,115,163]}$

$$
\begin{aligned}
& \mathrm{F}+\mathrm{h} v_{1} \stackrel{\mathrm{k}_{\mathrm{A}}}{\rightarrow} \mathrm{F}^{*} \stackrel{\mathrm{k}_{\mathrm{F}}}{\rightarrow} \mathrm{F}+\mathrm{h} v_{2} \\
& \mathrm{~F}+\mathrm{Q} \stackrel{\mathrm{k}_{\mathrm{FQ}}}{\longleftrightarrow}[\mathrm{FQ}] \stackrel{\mathrm{h} v_{\mathrm{Abs}}}{\longrightarrow}[\mathrm{FQ}]^{*} \rightarrow \mathrm{FQ}+\Delta
\end{aligned}
$$

Due to the peptide-bound position of the tryptophan, the imposed requirements on the quencher are versatile. Requirements of the quencher molecules are membranepermeability, a definite distance to the centre of the membrane and no change of the membrane properties. Here, especially lipid-bound quencher molecules have proven to be useful. As quencher unit, bromine ${ }^{[23]}$ atoms or DOXYL ${ }^{[20]}$ groups are incorporated into the acyl chains. 
In the following, the radical DOXYL was used as static quencher molecule for tryptophan. DOXYL can be covalently bound at defined positions of steric acid or 1-palmitoyl-2stearoyl-(X-DOXYL)-sn-glycero-3-phosphocholine (16:0-X DOXYL PC) lipids (see Figure 3.9). ${ }^{[164]}$

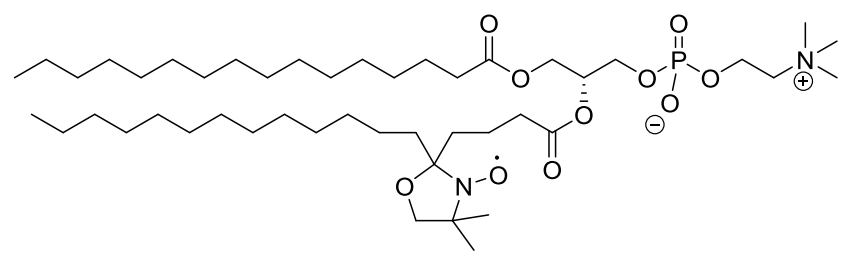

Figure 3.9 1-Palmitoyl-2-stearoyl-(5-doxyl)-sn-glycero-3-phosphocholine (16:0-5 DOXYL PC).

Because of the quencher's specific localisation in the lipid membrane, it is possible to establish a distance correlation between the quencher and fluorophore. This method is called the parallax method. By the variation of the quencher mole fraction $\chi_{\text {DOXYL }}$ and the resulting change in florescence intensity, the position of tryptophan can be calculated using the two-dimensional Perrin equation (see Equation 3.3). ${ }^{[24,114,115,163]}$

$$
\ln \frac{\mathrm{I}}{\mathrm{I}^{0}}=-\frac{\Pi \cdot\left(\mathrm{R}_{0}^{2}-\mathrm{X}^{2}-\mathrm{Z}^{2}\right)}{70} \cdot \chi_{\mathrm{DOXYL}}
$$

The critical quenching radius $\mathrm{R}_{0}$ and lateral distance between fluorophore and quencher $\mathrm{X}$ is presumed as a constant for the parallax calculation. The distance of the fluorophore and quencher is described by $\mathrm{Z}$ (see Figure 3.10). For the graphic analysis, the natural logarithm of tryptophan fluorescence in the present of quencher (I) divided by tryptophan fluorescence in the absence of quencher $\left(\mathrm{I}_{0}\right)$ was plotted against $\chi_{\text {DOXYL }}$. The resulting slope of the straight line is in direct proportion to $\left(\mathrm{R}_{0}^{2}-\mathrm{X}^{2}-\mathrm{Z}^{2}\right)$. The comparison of different graphic analysis with various DOXYL-labelled lipids has a known distance to the lipid bilayer centre $(\mathrm{Q})$ and allows the calculation of the distance of tryptophan to the lipid bilayer centre $(\mathrm{P})$.

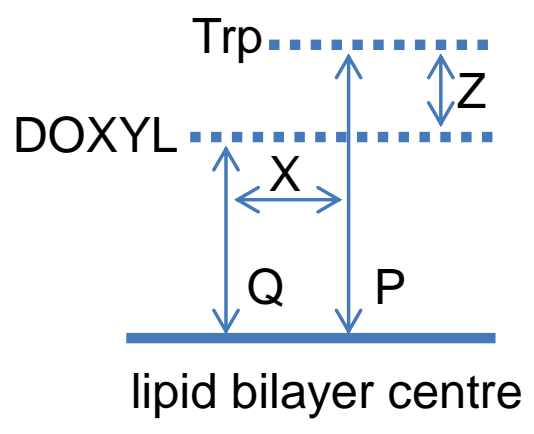

Figure 3.10 Graphical visualisation of the geometric parameters which are necessary for the calculation of the distance Trp/lipid bilayer centre $(P)$ : lateral distance between fluorophore and quencher $(X)$, distance of the fluorophore and quencher $(Z)$, distance of the quencher and lipid bilayer centre $(Q) .{ }^{[20]}$ Adapted with permission from L. A. Chung et al, Biochemistry 1992, 31, 6608-6616. Copyright (1992) American Chemical Society. 


\section{Measurements}

For the parallax measurements different LUV compositions were prepared. The used films were perpetrated with a constant lipid concentration $\left(n=0.3 \cdot 10^{-6} \mathrm{~mol}\right)$ of DOPC or a combination of DOPC and the corresponding DOXYL-labelled lipid. As DOXYL-labelled lipid 16:0-10 DOXYL PC and 16:0-16 DOXYL PC were used. The peptide/lipid concentration was 1:200 and the vesicles were rehydrated with ultrapure water.

In Figure 3.11 a section of the results from the parallax measurement of Pep 1 with 16:016 DOXYL PC (full results in Appendix C) is illustrated. The normalised fluorescents spectra show a strong dependence of the fluorescence intensity to the mole fraction of the quencher molecule $\chi_{\text {DOXYL }}$. Due to the spatial proximity, this behaviour was expected. The graphic display of $\ln \left(\mathrm{I} / \mathrm{I}_{0}\right)$ against $\chi_{\text {DoXYL }}$ creates a balance line with a negative slope. In a comparative manner to Figure 3.11, Figure 3.12 shows the correlation of Pep 1 and 16:0-10 DOXYL PC (full results in Appendix C). Here, the fluorescence intensity increased in small mole fraction of the 16:0-10 DOXYL PC. This behaviour was also observed during several measurements under the same conditions. Therefore, it is not possible to draw a balance line with a negative slope, which would be necessary for a meaningful evaluation of the distance measurements.

a)

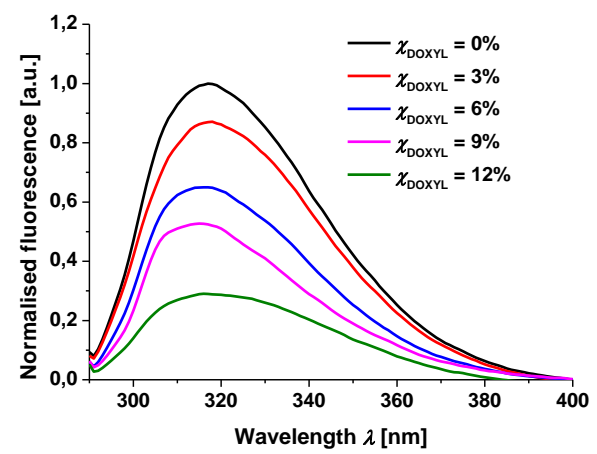

b)

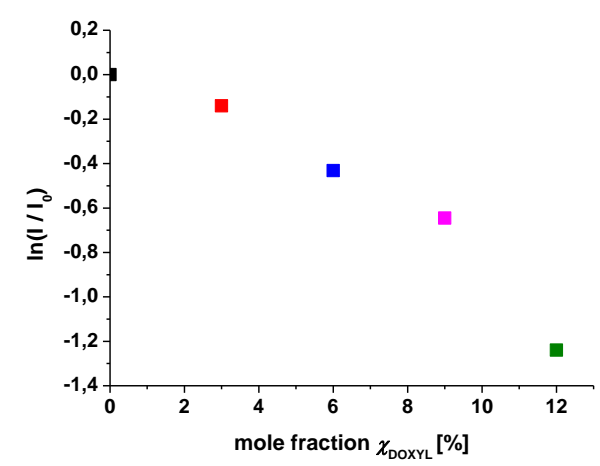

Figure 3.11 a) Normalised fluorescence spectra of Pep 1 in DOPC/16:0-16 DOXYL PC with changing lipid/quencher ratio. b) Fluorescence depth quenching curves derived from the corresponding fluorescence spectra (LUVs, P/L-ratio 1:200, $\left.T=25^{\circ} \mathrm{C}\right)$.

The measured peptides Pep 1 and Pep 2 display in general an expected course for the different DOXYL-labelled lipids (see Appendix C). Peptide Pep 1 with the central tryptophan tends to show a stronger quenching effect at 16:0-10 DOXYL PC and 16:0-16 DOXYL PC than peptide Pep 2, with the peripheral tryptophan. Furthermore, the quenching effect from Pep 1 at higher molar fractions $\chi_{\text {DOXYL }}$ of 16:0-16 DOXYL PC is greater than at 16:0-10 DOXYL PC. However, the measurements indicated fluorescence 
properties of peptides as described, which were unexpected. Based on these results, various lipid-peptide interactions might be responsible. An influence of the fluorophore or the fluorophore quencher complexes [FQ] is possible, which would result in changed fluorescence behaviour. It is known that fluorescence parameters, like wavelength maximum, fluorescence intensity, fluorescence lifetimes and band shape are influenced by the fluorophore's environment. ${ }^{[16-19,165]}$ The modified lipid composition might lead to a modification of the lipid-peptide interaction, what in return leads to a change in the tryptophan environment.

a)

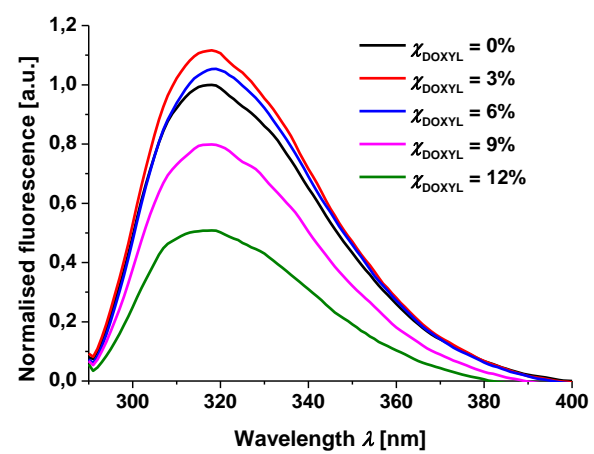

b)

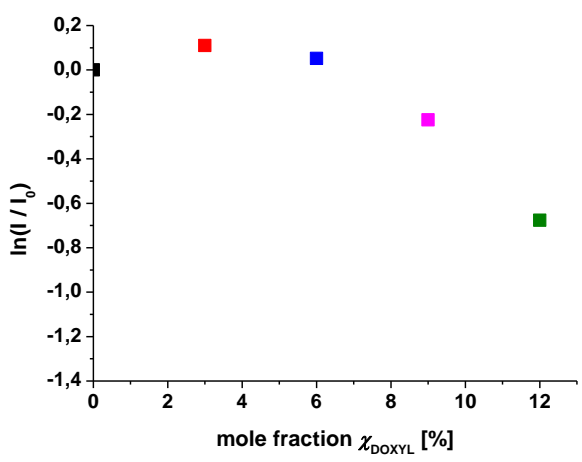

Figure 3.12 a) Normalised fluorescence spectra of Pep 1 in DOPC/16:0-10 DOXYL PC with changing lipid/quencher ratio. b) Fluorescence depth quenching curves derived from the corresponding fluorescence spectra (LUVs, P/L-ratio 1:200, $\left.T=25^{\circ} \mathrm{C}\right)$.

Possible reasons for this could be peptide aggregations or other lipid-peptide interactions (see Figure 2.6). A further source of error in this measurement might be the vesicle preparation. Although the vesicles were made several times, it could be that the incorporation of 16:0-10 DOXYL PC has led to a modified peptide insertion.

Based on results of RosT et al. with comparable peptides, where a heavy atom labelled peptide was incorporated into the bilayer and the exact position could been proven by $\mathrm{X}$-ray measurements, ${ }^{[38]}$ it is known that the synthesised peptides are transmembrane peptides. However, in this measurement the labelling with non-commercially available heavy atoms is necessary. The parallax method can be carried out with natural amino acids or with commercially available fluorophores. In further parallax measurements, a change in the experimental setup, such as a subsequent insertion of the peptides in the membrane, should be done. 


\subsection{Cyanide-labelled $\beta^{3}$-amino acid as molecular sensor for surface-enhanced infrared absorption (SEIRA)}

The measurement of transmembrane peptides in the natural environment is a demanding assignment. Though, important information can be obtained about the orientation, aggregation and localisation of transmembrane peptides in biological membranes. Hence, a lot of measurement techniques were developed and are still further improved. The lipid mesophase crystallisation, for example provides an opportunity to obtain information about the secondary structure of transmembrane peptides in a lipid like crystal surrounding. ${ }^{[158,159]}$ In contrast, for the measurement of dynamic systems infrared spectroscopy in the attenuated total reflection mode (ART-IR) was established. This is an enhanced IR technique, where the sample is in contact with an internal reflection element (IRE). Since this is a surface-technique, information about the peptide orientation or secondary structure in lipid bilayers can be analysed. ${ }^{[166-168]}$ A further development of the ATR-IR technique is the surface-enhanced infrared absorption (SEIRA) spectroscopy. Here, the IRE is covered by an metal-island film. ${ }^{[169-171]}$ In comparison to the ATR-IR technique SEIRA spectroscopy results in a higher enhancement and the molecules can react with the metal film and build self-assembled monolayers which is the simplest biomimetic membrane model. ${ }^{[26,27]}$

\subsubsection{Surface-enhanced infrared absorption (SEIRA)}

The biologically, chemically and physically crucial methods, ATR-IR and SEIRA have a very similar setup to each other. The probe will be examined by IR and is placed on a IRE. ${ }^{[27,167]}$ Total reflection is achieved at the interface and the electromagnetic field of the photon leads to a propagation of an evanescent wave through the interface. When a molecule is absorbed on the IRE, it can couple with the electric field of the wave, absorb energy and therefore attenuate the total reflection beam. SEIRA differs from ATR-IR by a thin nanostructured metal layer that separates the internal reflection element and the probe. ${ }^{[26-28]}$ This nanostructured layer increases the intensity of the IR spectrum up to three orders of magnitude, which results in a better resolution. ${ }^{[27]}$ The enhancement is due to an electromagnetic and chemical mechanism. The electromagnetic mechanism is based on plasmon resonance and a perturbation of the optical properties. Responsible for this is the chemical donor-aceptor interactions and charge-transfer effects. ${ }^{[169,171]}$ The enhancement decays from the surface to the solution. For this reason it is possible to enhance structures resolution up to $10 \mathrm{~nm}$ above the gold surface while signals over the $10 \mathrm{~nm}$ area, like the bulk solution are not enhanced. ${ }^{[27]}$ 
The IR based methods and the required variables for the following calculation are well known for the natural $\alpha$-peptides. ${ }^{[170]}$ For the more particular $\beta$-peptides some of the measurements needed new adjustments. To establish the usable measurement of $\beta$-peptides, some model peptides were crucial to be developed. The focus of this study was measuring the influence of an electrical stimulated membrane, as well as the influence on incorporated transmembrane $\beta$-peptides by SEIRA. The objective is to investigate peptide properties which can be used as a basis for further investigation methods.

The vibrational STARK effect is a promising tool to study electric fields in and at biomimetic interfaces. ${ }^{[172,173]}$ STARK reporter groups such as the nitrile group, react very sensitive to changes of the electric field around them and a frequency shift in the vibrational spectrum is observable. ${ }^{[174-178]}$ On this basis, SCHKOLNIK et al. was able to correlate the nitrile stretch vibration of 4-mercaptobenzonitrile in a locale field. ${ }^{[172]}$ The 4-mercaptobenzonitrile formed on the nanostructured gold surface self-assembled monolayer, thus builds the simplest biomimetic interface. ${ }^{[172]}$ The gold-coated surface in the SEIRA measurements makes it possible, among other things, to generate an electric field in direct proximity of the sample. In order to use this measurement for the investigation of $\beta$-peptide, it is necessary to synthesise an electrochemical sensitive amino acid with a STARK reporter group. Based on literature known $\alpha$-amino acids, a nitrile including $\beta$-amino acid was developed and incorporated in a model $\beta$-peptide. ${ }^{[179,180]}$ Due to this the following Fmoc-D- $\beta^{3}-\mathrm{Ala}(\mathrm{CN})-\mathrm{OH}$ amino acid was developed and the peptide Pep 11 was synthesised.

\subsubsection{Synthesis and measurements}

\section{Synthesis of cyanide-labelled amino acid and peptide}

Based on the structure of the desired molecule some different methods for the synthesis of the Fmoc-D- $\beta^{3}-\mathrm{Ala}(\mathrm{CN})-\mathrm{OH} 1$ would be possible (see Figure 3.13). A retrosynthetic analysis suggested as possible reaction routes a nucleophilic substitution or a dehydration reaction.

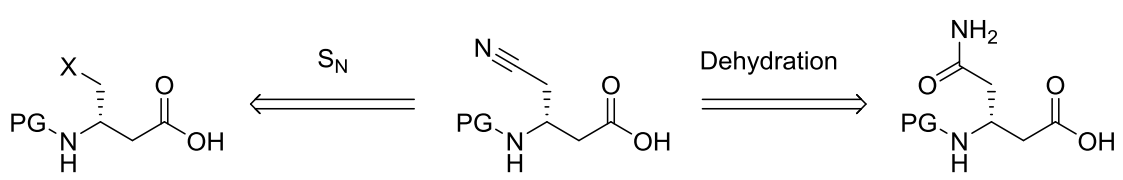

Figure 3.13 Retrosynthetic analysis of the $N$-protected target $\beta^{3}$-amino acid by a nucleophilic substitution or a dehydration reaction. $X$ illustrates a leaving group that can be exchanged by nitrile and PG represents an arbitrary protection group. 
Based on the work of ORTGIES et al. the nucleophilic substitution route was performed using Fmoc-D- $\beta^{3}$-Ser-OH (1.00 eq), which was activated at the free alcohol group by trimethylsilyl chloride (TMS-Cl, $2.40 \mathrm{eq})$ and potassium iodide $(2.40 \mathrm{eq})$ in ACN. ${ }^{[181]}$ The activated alcohol should be attacked in a nucleophilic substitution reaction by potassium cyanide (KCN, $3.00 \mathrm{eq}$ ), but mass spectrometric analysis did not show a successful conversion to the product. Allylic alcohol missing might be the reason for the unsuccessful conversion. Based on these results, another synthesis strategy was performed. A retrosynthetic analysis offered the possibility to receive the product by dehydration of asparagine. On the basis of these considerations two different dehydration reagents were tested with the analogue $\alpha$-amino acid Fmoc-Asn-OH. Firstly, a suspension of cyanuric chloride (1.00 eq) and Fmoc-Asn-OH (2.00 eq) in dry DMF was mixed, though no conversion to the desired product could be detected by mass spectrometry. Secondly, the dehydration reagent propylphosphonic anhydride $\left(\mathrm{T}^{\mathrm{P}} \mathrm{P}^{\circledR}\right)$ (1.00 eq) in ACN/EE (1:2) was added to Fmoc-Asn-OH (1.00 eq) and the desired $\alpha$-amino acid target product was confirmed in mass spectrometric analysis. $\mathrm{T}^{\circledR} \mathrm{P}^{\circledR}$ is a versatile molecule that can be used in many reactions, e.g. the transfer of aldehyde groups to nitrile groups or as a condensation reagents for peptide coupling. ${ }^{[182,183]}$ First of all, for the synthesis of the $\beta$-amino acid target product, the used $\alpha$-amino acid has to be transformed in a $\beta$-amino acid (see Scheme 3.1). Therefore, the established procedure of the ARNDT-EISTERT homologation (see Chapter 2.4) was performed using Fmoc- $\alpha-\mathrm{D}-\mathrm{Asn}(\mathrm{Trt})-\mathrm{OH}$ 2. Afterwards the trityl side chain protection group was cleaved under acid conditions (TFA/ $\mathrm{H}_{2} \mathrm{O} / \mathrm{EDT}$ 90:5:5) followed by a dehydration with $\mathrm{T}^{\circledR} \mathrm{P}^{\circledR}$. The product Fmoc-D- $\beta^{3}-\mathrm{Ala}(\mathrm{CN})-\mathrm{OH} 1$ was obtained over three steps with an overall yield of $26 \%$. With this component the following peptide Pep 11 (see Figure 3.14) was synthesised by manual microwave-supported SPPS without changing the coupling method for Fmoc-D- $\beta^{3}$-Ala $(\mathrm{CN})-\mathrm{OH}$ (see Chapter 2.5.1).

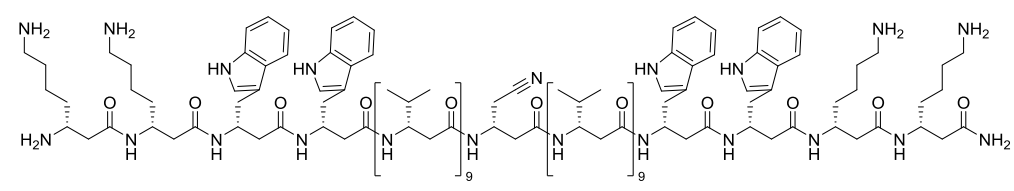

Figure 3.14 Sequence of peptide Pep 11.

Pep 11 


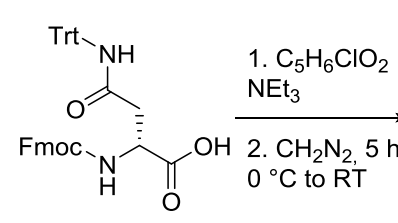

2

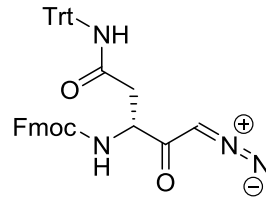

3

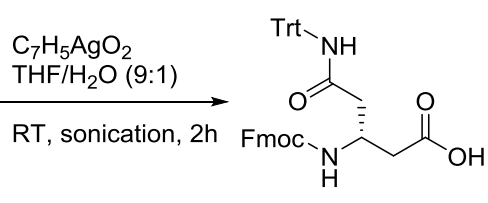

4

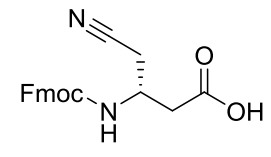

$26 \%$ (over 3 steps)

1

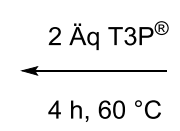

$4 \mathrm{~h}, 60^{\circ} \mathrm{C}$

Scheme 3.1 Generation of the Fmoc-protected amino acid 1 with an overall yield of $26 \%$.

After cleavage from the resin (without any purification), the mass spectrometric analysis of the peptide showed two signals with a similar intensity. The target peptide Pep 11 was identified with a mass of $3531.4 \mathrm{~g} / \mathrm{mol}$. Additionally, a water adduct $\left[\mathrm{M}+\mathrm{H}_{2} \mathrm{O}\right]$ was identified with a mass of $3549.4 \mathrm{~g} / \mathrm{mol}$. There are two explanations for this result. Firstly, the used amino acid was not fully purified and remaining Fmoc- $\beta$-D-Asn-OH was incorporated during the SPPS. However, a contamination of the used amino acid can be excluded within the resolution limits of the NMR spectroscopy. The ${ }^{1} \mathrm{H}$ - and ${ }^{13} \mathrm{C}-\mathrm{NMR}$ spectra show no characteristic signal of the amide group. An amide group (150 - $190 \mathrm{ppm})$ shows in a ${ }^{13} \mathrm{C}-\mathrm{NMR}$ spectrum a stronger downfield shift relative to a cyanide group $(105-130 \mathrm{ppm}) .{ }^{[184]}$ In Figure 3.15 the ${ }^{13} \mathrm{C}-\mathrm{NMR}$ spectrum of the purified building block is shown. In the region of $150-190$ ppm only the carbon acid signal is displayed.

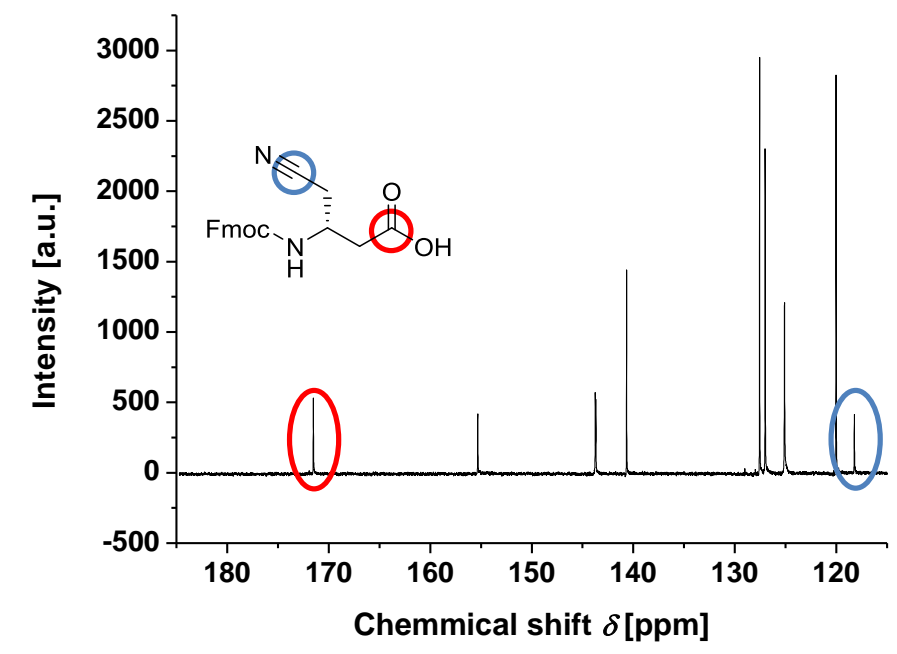

Figure 3.15 ${ }^{13} \mathrm{C}$-NMR spectrum (126 MHz, [D6] DMSO) of 1. 
Secondly, an addition of water happened during the cleavage of the peptide from the resin. This side reaction during the cleavage can be counteracting by lower temperature and less time during the cleavage step or the omitting of water in the cleavage cocktail. Nevertheless, the main product Pep 11 of this synthesis could be separated from $\left[\mathrm{M}+\mathrm{H}_{2} \mathrm{O}\right]$ by HPLC.

\section{Conformation and fluorescence studies}

The peptide Pep 11 was measured by CD spectroscopy in organic solvents (MeOH, TFE) and in phospholipids (DMPC, DPPC, DOPC). It was suspected that Pep 11 will form a 14-helix like Pep 1 - Pep 4 and Pep 9-Pep 10. The CD spectra of Pep 11 in organic solvent (TFE, $\mathrm{MeOH} \sim 50 \mu \mathrm{M}, T=25^{\circ} \mathrm{C}$ ) and in model lipid vesicle DMPC, DOPC, DPPC (LUVs, P/L-ratio $=5: 100, \quad T_{\mathrm{DMPC}}=30^{\circ} \mathrm{C}, \quad T_{\mathrm{DOPC}}=25^{\circ} \mathrm{C}, \quad T_{\mathrm{DPPC}}=50^{\circ} \mathrm{C}$ ) shows $\mathrm{a}$ minimum between $185 \mathrm{~nm}$ and $195 \mathrm{~nm}$ and a maximum between $200 \mathrm{~nm}$ and $220 \mathrm{~nm}$ (see Appendix C).

The fluorescence emission maxima $\lambda_{\max }$ of Pep 11 in the organic solvents and membrane environments showed a maximum at $\lambda_{\max }$, Pep $11=342 \mathrm{~nm}$ in $\mathrm{MeOH}$ respectively at $\lambda_{\max , \text { Pep 11 }}=346 \mathrm{~nm}$ in TFE and at $\lambda_{\max }$, Pep 11 $=338 \mathrm{~nm}$ in lipid environment (DMPC, DOPC, DPPC). This agrees with the previously presented results (see Chapter 3.2). The observed D- $\beta^{3}$-hTrp emission could be explained by their position within the peptide. This may not be as meaningful as in Pep 1-Pep 10, but previous studies showed an incorporation of a comparable peptide in DOPC bilayer by RosT et al. ${ }^{[38]}$ On the basis of the comparable florescence spectra, an incorporation of the peptide is assumed. Even though the peptides show similar results like comparable peptides, further investigations are still required. Studies have shown that nitrile derivatives of amino acids are dependent on the environment. ${ }^{[179,180]}$ In contrast to tryptophan, which shows a changed fluorescence behaviour, the nitrile derivatives show a shift in the $\mathrm{CN}$ stretching vibration. Based on the $\mathrm{CD}$ spectroscopic, it can be assumed that the incorporation of Fmoc-D- $\beta^{3}-\mathrm{Ala}(\mathrm{CN})-\mathrm{OH}$ has no influence on the secondary structure or the fluorescence behaviour of the $\beta$-peptide.

\section{IR- measurements}

In the IR spectroscopy asymmetrical vibrations are detected, which have a change in their transition dipole moment during the vibration. For the examination of proteins and peptides, the vibrations of the amide group are used. Hence, the most important vibrations are the amides A, B, I, II and III. The amide A $\left(3310-3270 \mathrm{~cm}^{-1}\right)$ and B $(3010-$ $\left.3030 \mathrm{~cm}^{-1}\right)$ are the result of the NH stretching. The amide I $\left(1700-1600 \mathrm{~cm}^{-1}\right)$ shows the 
CO stretching with a small amount of NH bending. ${ }^{[185]}$ The amide II $\left(1580-1480 \mathrm{~cm}^{-1}\right)$ and the amide III $\left(1300-1230 \mathrm{~cm}^{-1}\right)$ vibrations are a combination of the NH bending and $\mathrm{CN}$ stretching together with different proportions of the respective vibrations (see Figure 3.16). ${ }^{[185]}$ The amid I vibration is often affected by the vibrations of water $\left(\sim 1645 \mathrm{~cm}^{-1}\right)$. This interference of the $\mathrm{HOH}$ bending can be a big problem in many measurements of peptides and proteins. ${ }^{[185]}$
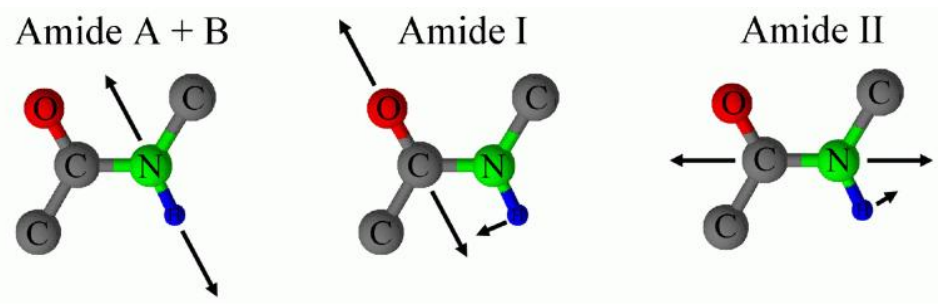

Amide III

Figure 3.16 Vibration types of the amide bond in the peptide backbone. ${ }^{[185]}$ Reprinted from J. A. Kozuch, Structure-Function Relationships of Membrane Proteins - Spectroelectrochemical Investigation of Artificial Membranes, 2013.

The peptide Pep 11 was examined by STAFFA from the HILDEBRANDT group (unpublished results). The properties of Pep 11 in the membrane were investigated by the amid I and the amid II vibrations. For this purpose it was tested to incorporate Pep 11 into a membrane with two different procedures. The two methods differ in the previous or subsequent incorporation of the peptides into the membrane. As the first method, the peptide was incorporated into LUVs and then spread to a self-assembled monolayer (SAM) consisting of dihydrocholesteryl (2-(2-(2-ethoxy)ethoxy)ethanethiol (WK3SH) and 6mercaptohexanol $(6 \mathrm{MH})$. Following this method it was possible to recognise the amide I and amid II vibrations (Figure 3.17 a). A CN vibration band in the range of $2500 \mathrm{~cm}^{-1}$ was not observed.

a)

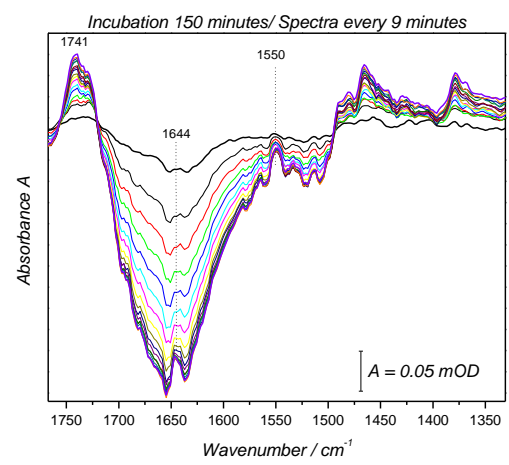

b)

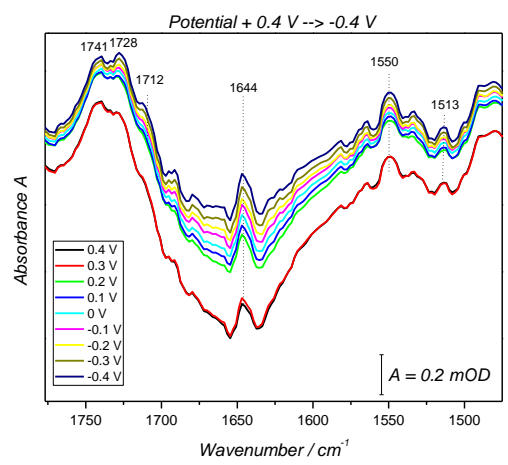

Figure 3.17 a) IR difference spectra of the incubation of LUV for $150 \mathrm{~min}$. Spectra were shown for every 9 min. The black spectrum is for $t=0 \mathrm{~min}$ and the violet for $t=150 \mathrm{~min}$. The high negative band at $1644 \mathrm{~cm}^{-1}$ belongs to a water vibration and the band at $1741 \mathrm{~cm}^{-1}$ belongs to the $C O$ vibration of the lipids. The band at $1644 \mathrm{~cm}^{-1}$ and $1550 \mathrm{~cm}^{-1}$ represent the Amid I and II vibrations. b) Potentials were applied for 9 min and spectra was detected from $0.4 \mathrm{~V}$ to $-0.4 \mathrm{~V}$. Small changes in the intensity can be observed, due to a decrease in the water vibration band. Measurements and graphics performed by STAFFA. 
After washing with buffer to get rid of the unbound vesicles, voltage was applied to the gold surface and showed no change in the ratio or intensity of the observed vibrations (Figure 3.17 b). Such a change, would have to be observed during a movement or restructuring of the peptide in the membrane. In the second method, the membrane was first built up on the SAM and subsequently the peptide was added. For this, the peptide was dissolved in $\mathrm{MeOH} / \mathrm{buffer}$ and added to the spreaded membrane. The concentration of organic solvent did not exceed $5 \%$, as this would destroy the membrane. Earlier studies by STAFFA showed that this type of preparation leads to a decrease in the water vibration in the spectrum. No amid vibration was observed, which indicates that there was no insertion of the peptide into the membrane or dissolving in the $\mathrm{MeOH} /$ buffer mixture. Summarising, it can be said that the synthesis of the cyanide amino acid building block was successfully accomplished and the incorporation into a transmembrane peptide by microwave-supported SPPS is possible. The measurements with SEIRA did not show successful results so far and have to be optimised. 


\section{Transmembrane $\beta$-peptides as anchor for near-membrane ion-sensors to study the concentration of $\mathrm{H}^{+}$and $\mathrm{Ca}^{2^{+}}$}

Biomolecular membranes represent a complex system that fulfil various tasks in cells. ${ }^{[1]}$ The polar and charged surface act as a focal point for cations. ${ }^{[186-188]}$ In particular, the interaction of protons $\left(\mathrm{H}^{+}\right.$, see Chapter 4.1) and calcium ions $\left(\mathrm{Ca}^{2+}\right.$, see Chapter 4.2) play an important role in research. ${ }^{[186-188]}$ As a new approach to measure the ion concentration at the membrane surface, $\beta$-peptide anchored $\mathrm{H}^{+}$- and $\mathrm{Ca}^{2+}$-sensors were synthesised, providing the advantages of a stable and easily predictable system.

\subsection{Near-membrane $\mathrm{H}^{+}$-sensor anchored by transmembrane $\beta$-peptides}

\subsubsection{Introduction and objectives}

Lipids, as the basic unit of the membrane, consist of a non-polar tail and a polar headgroup. Due to the amphipathic character the lipids form lipid bilayers. The headgroups can be subdivided into polar-neutral, zwitterionic or ionic groups. ${ }^{[1,189]}$ Lipid bilayers in aqueous systems direct their polar headgroups to the aqueous phase, which allows polar interaction and partially charged headgroups with ions in the aqueous phase. ${ }^{[1,186-189]}$ In addition to biologically important metal ions, the interactions of $\mathrm{H}^{+}$with the membrane surface are of interest.

The near-membrane $\mathrm{pH}$ value is an important indicator of cellular processes and an important driving force for processes located near biological membranes. On the one hand, the extracellular acidity for instance can be an indication for pathological states like tumours and infections or wounds. ${ }^{[190-193]}$ On the other hand, cellular energy production in the form of ATP is based on the generation of a $\mathrm{H}^{+}$gradient (see chapter 4.1.2). ${ }^{[1,194]}$ For the near-membrane $\mathrm{pH}$ value, it is necessary to understand the interaction of the ubiquitous $\mathrm{H}^{+}$with the membrane surface and therefore, it is still the subject of current studies with various experiential setups. ${ }^{[33,186,195]}$ The determination of the $\mathrm{pH}$ value was carried out with microelectrodes, ${ }^{[196-200]} \mathrm{pH}$ sensitive peptides $^{[201]}$ or $\mathrm{pH}$ sensitive fluorophores. ${ }^{[29,186,190,195,202,203]}$ The measurement by microelectrodes requires a fixed system to provide a consistent distance to the membrane and has a high equipment requirement. As an alternative, measurements with modified peptides were developed. For example, a modified gramicidin peptide was developed as a label free $\mathrm{pH}$-sensor. ${ }^{[201]}$ Here, the $C$-terminal $\mathrm{pH}$ insensitive ethanolamine is modified with a $\mathrm{pH}$ sensitive ethylenediamine or histamine. This leads to a protonation/deprotonation of the $C$-terminal group and 
therefore a cis/trans isomerisation is possible. ${ }^{[201]}$ This isomerisation controls the ion flow through the ion channel and thereby the single-channel currents. A low $\mathrm{pH}$ increases the permeability and a high $\mathrm{pH}$ decreases the permeability of the ion channel. ${ }^{[201]}$ Nevertheless, the larger percentage of near-membranes $\mathrm{pH}$ measurement is performed by fluorescence-based methods. The use of labelled molecules offers the possibility to observe a free moving system. Figure 4.1 depicts some fluorophores, which are used to measure the near-membrane $\mathrm{pH}$ value in model systems or cells. ${ }^{[29,34,190,203]}$ The required fluorophores can be attached to peptides as well as to lipids, which allows a wide range of applications. Especially for membrane studies, lipid coupled $\mathrm{pH}$ sensitive fluorophores are an important tool and for this purpose, the fluorophore is often bound to a phosphoethanolamine (PE) headgroup. ${ }^{[31,34,203,204]}$ Attaching fluorophores to the membrane via lipids prevents an influence by lipid-peptide interactions. Furthermore, the anchoring by lipids enables a simplified variation of the alkyl chain. This allows for example a better adaptation in different membrane thickness. ${ }^{[205]}$ The natural range of lipids offers only a small variation of the headgroups, which can be used for this purpose.

a)

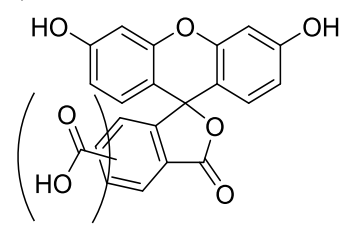

b)

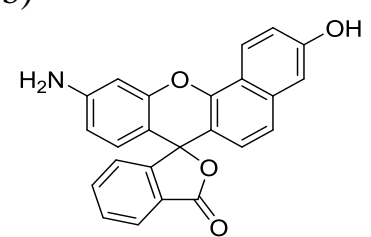

c)

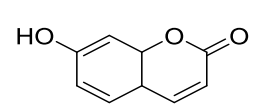

Figure 4.1 Examples of pH sensitive molecules that are used to detect near-membrane pH changes. a) Fluorescein and in brackets its derivate carboxyfluorescein, b) seminaphtharhodafluor (SNARF), c) 7-hydroxychromen-2one. ${ }^{[29,34,190,203]}$

However, due to their special properties, peptides can facilitate certain methods of measurement. ANDERSON et al. labelled a pH low insertion peptide, which inserts itself in a cell membrane depending on the $\mathrm{pH}$ value. ${ }^{[190]}$ This system with seminaphtharhodafluor (SNARF, Figure 4.1) as the fluorophore was able to detect a $\mathrm{pH}$ difference of $0.2-0.3$ units.

As an unexplored possibility of membrane anchoring, the usage of $\beta$-peptides is presented in this work. For this purpose, already known $\beta$-peptide transmembrane structures were expanded with hydrophilic elongation and modified with carboxyfluorescein (see Chapter 4.1.4). These $\beta$-peptides allow the development of a modifiable and predictable structure (see Chapter 2.1). 


\subsection{2 $\mathrm{H}^{+}$Behaviour at the membrane interface}

Despite the simple structure of $\mathrm{H}^{+}$and $\mathrm{OH}^{-}$, the basic properties are part of many studies. ${ }^{[186]}$ In particular, the behaviour of $\mathrm{H}^{+}$on biological membranes differs from the behaviour in aqueous solutions. In buffered aqueous systems, the transport of $\mathrm{pH}$ relevant ions takes place by the buffer molecules. ${ }^{[186]}$ This transport is a rather slow process in relation to other biological relevant $\mathrm{H}^{+}$transports. Especially for biological ATP synthesis or the active transport of molecules across the membrane driven by a $\mathrm{H}^{+}$gradient, a slow buffer mediated diffusion would be disadvantageous. ${ }^{[206]}$ Biological processes driven by a $\mathrm{H}^{+}$gradient are generally performed in a two-step process. ${ }^{[1]}$ As a first step the conversion of energy (chemical or physical) by specialised transmembrane proteins leads to an active $\mathrm{H}^{+}$transport across the membrane, which results in the formation of a $\mathrm{H}^{+}$gradient. As a second step, this gradient can be used for synthesising ATP or as driving force for the active transport of molecules through the membrane. ${ }^{[1]}$ The peptides that are necessary for the generation of the $\mathrm{H}^{+}$gradient are also localised in the membrane. ${ }^{[1]}$ The exact $\mathrm{H}^{+}$ pathway between the gradient creating and the gradient exploited peptides, is still unclear. ${ }^{[186,207]}$ After the transport of the $\mathrm{H}^{+}$through the membrane in general, two options are possible. Firstly, a rapid equilibration with the bulk solution occurs, which results in the loss of the $\mathrm{H}^{+}$to the bulk solution (see Figure 4.2 a) or secondly, a membrane located $\mathrm{H}^{+}$diffusion occurs which allows buffering and transport via the membrane (see Figure $4.2 \mathrm{~b}) .{ }^{[208]}$

a)

b)

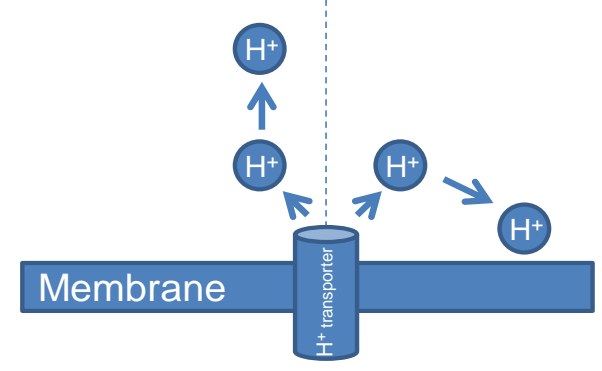

Figure 4.2 After the transport trough a $\mathrm{H}^{+}$transporter two different pathways are possible. a) $\mathrm{The}^{+}$can be equilibrate with the bulk solution or b) the $\mathrm{H}^{+}$can be localised at the membrane water interface.

Studies have shown that the affinity of $\mathrm{H}^{+}$to a membrane/water interface is larger than to an air/water interface. ${ }^{[186]}$ These differences in affinity are due to the polar or charged headgroups, and to the great hydration of the lipids. XU et al. from the WIDENGREN group determined using fluorescence correlation spectroscopy (FCS), a critical surface area for a $\mathrm{H}^{+}$-collecting antenna effect of membranes. ${ }^{[33,208]}$ Previous studies from the BRZEZINSKI 
and WIDENGREN group have shown that labelled lipid headgroups incorporated in membranes show a similar $k_{\text {off }}$ value (see Figure 4.3). ${ }^{[31,32,34,208]}$ In contrast, the value of the $k_{\mathrm{on}}$ is critically determinative by the environment of the fluorophore. Compared with fluorescein dissolved in water, lipid-bound fluorescein shows a 100-fold higher $k_{\text {on }}$ value. ${ }^{[208]} \mathrm{XU}$ et al. investigated so-called lipid nanodisc with different diameter sizes in their study (Figure 4.3).

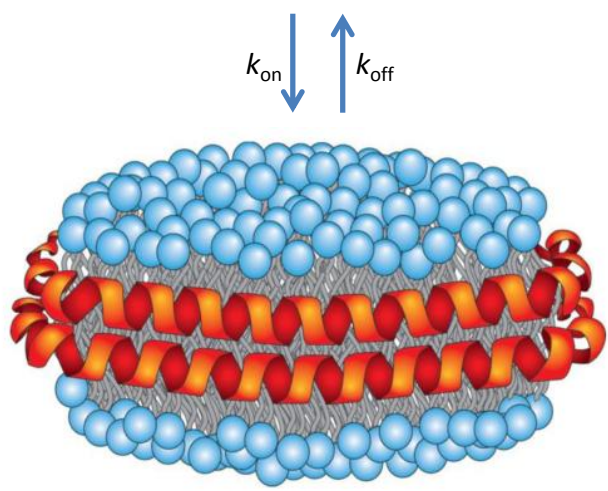

Figure 4.3 Schematic depiction of a lipid nanodisc generated by membrane scaffold proteins. ${ }^{[209]}$ Adapted with permission from I. G. Denisov et al, Chem. Rev. 2017, 117, 4669-4713. Copyright (2017) American Chemical Society.

These nanodiscs showed with a surface size less than $60 \mathrm{~nm}^{2}$ a $k_{\text {on }}$ that corresponds to water-soluble fluorescein. Above $60 \mathrm{~nm}^{2}$ the $k_{\text {on }}$ shows a large increase, which is more independent than the surface size. ${ }^{[33]}$ Among other studies, this provides a hint of the $\mathrm{H}^{+}$-capturing effect of the membrane.

However, the precise motion of a $\mathrm{H}^{+}$transported over the membrane, like from a transporter to an ATP synthase is still being discussed. ${ }^{[186,206-208]}$ For a better understanding of $\mathrm{H}^{+}$dynamics at the membrane/water interface, studies have been conducted on lipid monolayers, bacterial membrane fragments, lipid nanodiscs and lipid bilayers. ${ }^{[186]}$ These experimental studies were partly supported by theoretical considerations. ${ }^{[186]}$ In particular by experiments on lipid monolayers and on membrane fragments of purple bacteria, two different hypotheses emerged for the $\mathrm{H}^{+}$movement over the membrane. ${ }^{[186]}$ In the first hypothesis it is assumed that the $\mathrm{H}^{+}$spread through a hop between titratable membrane parts. The titratable membrane parts are primary lipids, which can take up and release $\mathrm{H}^{+}$, what result in an lateral $\mathrm{H}^{+}$movement (see Figure 4.4 a). The second hypothesis assumes that the near-membrane water phase acts as a hydrogen bridge network. The transport takes place between the titratable membrane parts and the water borderline (see Figure 4.4 b). ${ }^{[186]}$ 
a)

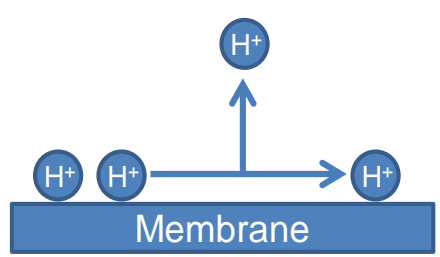

b)

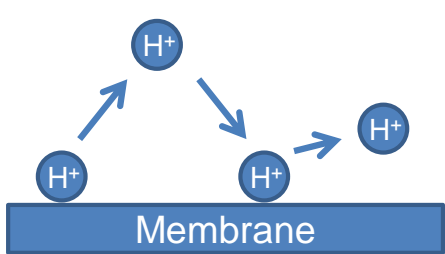

Figure 4.4 Schematic representation of a) irreversible and b) quasi equilibrium $\mathrm{H}^{+}$distribution over the membrane. ${ }^{[186]}$ Adapted with permission from N. Agmon et al, Chem. Rev. 2016, 116, 7642-7672. Copyright (2016) American Chemical Society.

However, there is still no clear evidence for either mechanism. Taking the abovementioned studies partly into account, GENNIS provided a few simplified conclusions for the $\mathrm{H}^{+}$dynamics at membrane surfaces. ${ }^{[208]} \mathrm{He}$ described that biological membranes have a narrow area at the membrane/water interface with increased $\mathrm{H}^{+}$concentration in relation to the bulk solution. These hydrated $\mathrm{H}^{+}$are stabilised by headgroup phosphate groups as well as carbonyl groups and $\mathrm{H}^{+}$stabilisation results in an increased near-membrane $\mathrm{pH}$. This behaviour was observed in zwitterionic and negative charged lipids. ${ }^{[186,208]}$ The transport of $\mathrm{H}^{+}$takes place through a deep interface zone by the lateral diffusion of lipids and the transport along near-membrane water clusters (see Figure 4.5). ${ }^{[208]}$

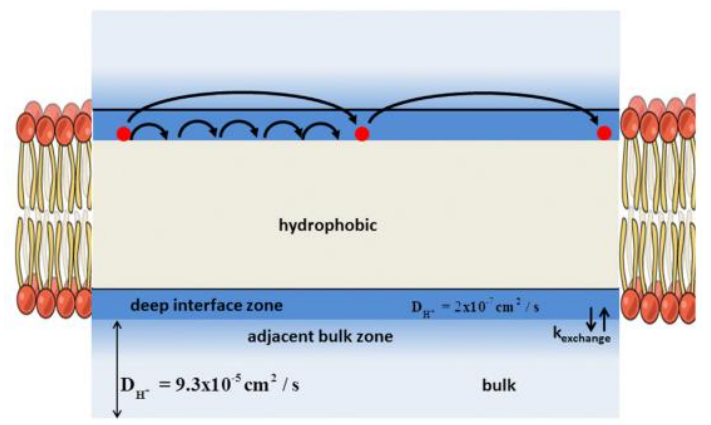

Figure 4.5 Schematic $\mathrm{H}^{+}$movement in deep interface zone and adjacent bulk zone by GENNIS. ${ }^{[208]}$ Reprinted from Biophys. J., 110 (9), R. B. Gennis, Proton Dynamics at the Membrane Surface, 1909-1911, Copyright (2016), with permission from Elsevier.

As an indication that there are several processes for $\mathrm{H}^{+}$transport, the $\mathrm{H}^{+}$diffusion coefficient was determined by FCS. This diffusion coefficient is with $D_{\mathrm{S}}=2 \times 10^{-7} \mathrm{~cm}^{2} \mathrm{~s}^{-1}$ round 2-3-fold faster than the diffusion coefficient of phospholipids in the lipid bilayer. ${ }^{[208]}$ In addition to effects not described above, the following statements about the buffer concentration and the $\mathrm{H}^{+}$flux are provided by GENNIS. ${ }^{[208]}$

1. The transport of $\mathrm{H}^{+}$across the membrane is comparatively faster in relation to the exchange with the bulk solution. ${ }^{[208]}$

2. At a low concentration of buffer $(<10 \mathrm{mM})$, the distribution of $\mathrm{H}^{+}$in the bulk solution after leaving the deep interface zone is slow. ${ }^{[208]}$ 
3. The $\mathrm{H}^{+}$tend to move in the adjacent bulk zone and back again to the membrane surface. $^{[208]}$

4. At a higher buffer concentration $(>10 \mathrm{mM})$, a faster distribution of the $\mathrm{H}^{+}$with the bulk solution takes place and a membrane surface acidification does not take place. $^{[208]}$

There are several equilibriums for the movement of $\mathrm{H}^{+}$along unilamellar vesicles, which should be taken into account when using a membrane-bound $\mathrm{pH}$ active fluorophore. The unilamellar vesicles represent a common model system for biomembranes. SANDÉN et al. postulate the following equations for the investigation of a lipid-bound $\mathrm{pH}$ sensitive fluorophore by FCS. ${ }^{[31]}$

$$
\begin{gathered}
\mathrm{F}^{-}+\mathrm{H}^{+} \underset{\mathrm{k}_{-1}}{\stackrel{\mathrm{k}_{1}}{\rightleftarrows} \mathrm{FH}} \\
\mathrm{F}^{-}+\mathrm{LH} \underset{\mathrm{k}_{2}}{\stackrel{\mathrm{k}}{\rightleftarrows}} \mathrm{FH}+\mathrm{L}^{-} \\
\mathrm{L}^{-}+\mathrm{H}^{+} \underset{\mathrm{k}_{-3}}{\stackrel{\mathrm{k}_{3}}{\rightleftarrows} \mathrm{LH}}
\end{gathered}
$$

These equations describe the protonation of the fluorophore (F) with the bulk solution (Equation 4.1), the exchange of $\mathrm{H}^{+}$between the lipids (L) and the fluorophore (Equation 4.2), as well as the exchange between lipids and the bulk solution (Equation 4.3, see Figure 4.6). ${ }^{[31]}$ In the same study, SANDÉN et al. examines the influence of ion concentration on the properties of lipid-bound fluorophores. Measurements with different ion concentrations make it possible to deduce the chemical potential $\Psi$ and the distance $\mathrm{x}$ between the fluorophore and the membrane surface (see Figure 4.6). ${ }^{[31]}$ KRAAYENHOF et al. developed various fluorophores anchored in the membrane by alkyl chains. ${ }^{[29]}$ With these coumarin-based fluorophores it is possible to measure the $\mathrm{H}^{+}$concentration at different distances from the membrane by $\mathrm{pH}$ titration. The difference in distance is achieved by using various linkages (see Figure 4.7). ${ }^{[29]}$ 


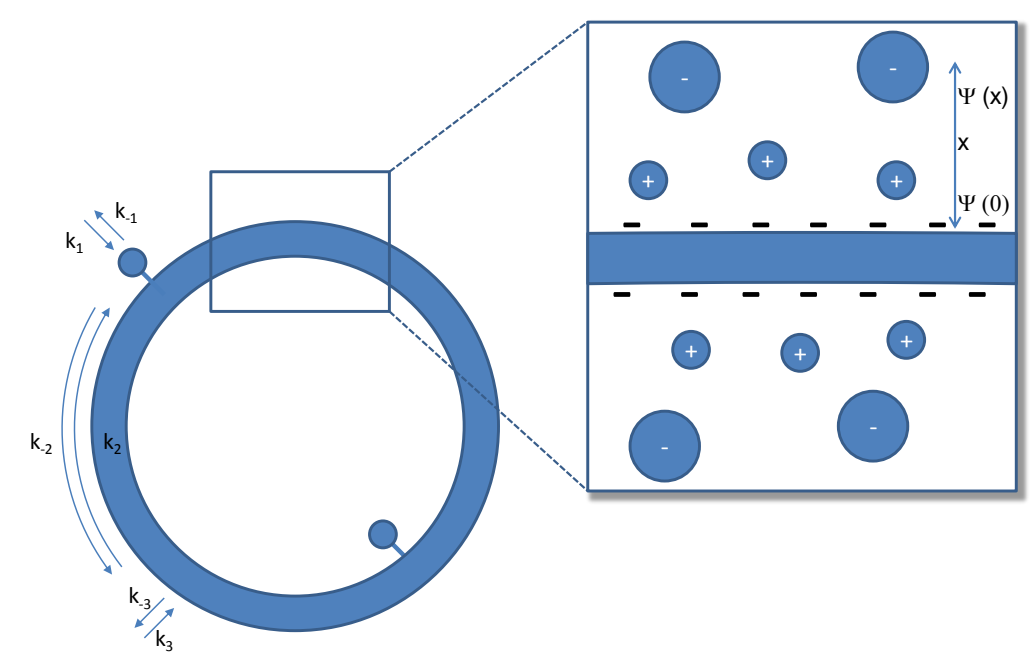

Figure 4.6 Illustration of the different equilibrium reactions at the membrane surface, when measured with a fluorophore labelled lipid. Graphic in the showcase: Ion distribution at the membrane and chemical potential $\Psi$ with distance $x$ from the membrane surface. ${ }^{[31]}$

Four fluorophores were used, which have an average distance to the membrane of $0.2 \mathrm{~nm}$ (U-2), $0.6 \mathrm{~nm}(\mathrm{U}-6), 1.0 \mathrm{~nm}(\mathrm{U}-10)$ and $1.3 \mathrm{~nm}(\mathrm{U}-13)$. Despite repulsion forces, based on the positive charge of the coumarin based fluorophores and the positive charged linkers, there are several degrees of freedom in the structure. ${ }^{[29]}$ According to SANDÉN et al., the distance between the fluorophore and the membrane can be strongly influenced by the ion concentration, especially when the linker between anchor and fluorophore is very flexible (see Figure 4.6). ${ }^{[31]}$

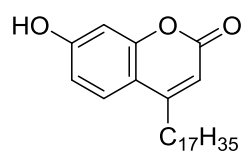

$\mathrm{U}-2$<smiles>CN(C)Cc1cc(=O)oc2cc(O)ccc12</smiles>

U-6

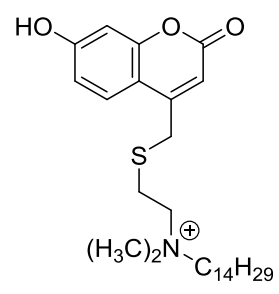

$\mathrm{U}-10$

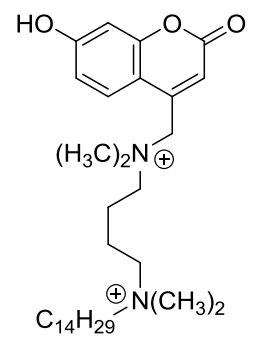

$\mathrm{U}-13$

Figure 4.7 Chemical structure of membrane-bound pH-sensors synthesised by KRAAYENHOF with different distances to the membrane surface (distance to the surface from left to right $0.2 \mathrm{~nm}, 0.6 \mathrm{~nm}, 1.0 \mathrm{~nm}$ and $1.3 \mathrm{~nm})^{[29]}$

However, the results of KRAAYENHOF et al. confirm a change in the titration behaviour of $\mathrm{pH}$ active fluorophores by different distance to the membrane surface and the membrane composition. $^{[29]}$

\subsubsection{Carboxyfluorescein as pH-sensor}

A large number of indicators for measuring the $\mathrm{pH}$ are available. However, for measuring the intracellular $\mathrm{pH}$ only a small number of dyes are available because certain requirements 
have to be fulfilled. ${ }^{[210]}$ The dyes must be non-toxic to the cell, not interfere with any cellular process or alter cellular properties. In Figure 4.8 some typical used $\mathrm{pH}$ sensitive dyes are given. ${ }^{[210]}$

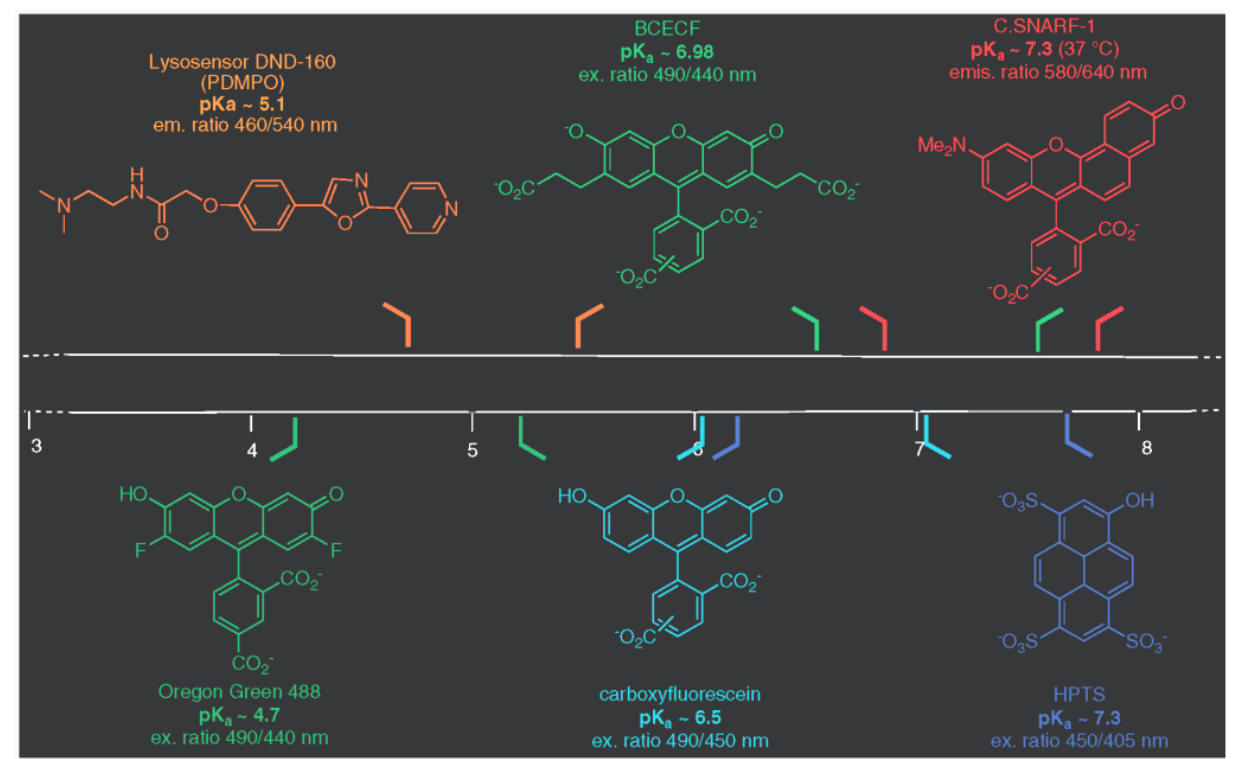

Figure 4.8 Examples of the pH range of typical pH sensitive dyes. ${ }^{[210]}$ Reprinted with permission from J. Han et al, Chem. Rev. 2010, 110, 2709-2728. Copyright (2010) American Chemical Society.

Furthermore, $\mathrm{pH}$ active fluorophores should not interact with the hydrophobic part of the membrane when they have to be used near the membrane. Depending on the way of anchoring in the membrane, an undesired interaction can lead to membrane deformation or an incorporation of the molecule into the membrane. HUGHES et al. examined some of the most common water-soluble fluorophores and their interaction with model lipid bilayers. ${ }^{[211]}$ Here, the $\mathrm{pH}$ sensitive molecule carboxyfluorescein was investigated among other fluorophores. It was found that carboxyfluorescein has a membrane interaction factor (mif) less than 0.1, which stands for almost no interaction with the membrane. The low interaction with the membrane is confirmed by studies, in which carboxyfluorescein-labelled lipids were used as lipid headgroup fluorescent label. ${ }^{[33,212-214]}$ Based on these results and the good commercial availability, carboxyfluorescein is a promising $\mathrm{pH}$-sensor for near-membrane $\mathrm{pH}$ measurements.

Carboxyfluorescein differs from the basic structure fluorescein, by the addition of a carboxyl group at the (5) or (6) position (see Figure 4.1). Commercially available are the pure conformers as well as a mixture of both (61:39 isomer ratio). ${ }^{[215]}$ The additional carboxylic acid at position (5) or (6) allows a connection to a molecule without major changes in the fluorophore system. Due to multiple ionic forms, carboxyfluorescein possesses several $\mathrm{p} K_{\mathrm{a}}$ values and different UV/Vis spectra (see Figure 4.9). ${ }^{[216]}$ 


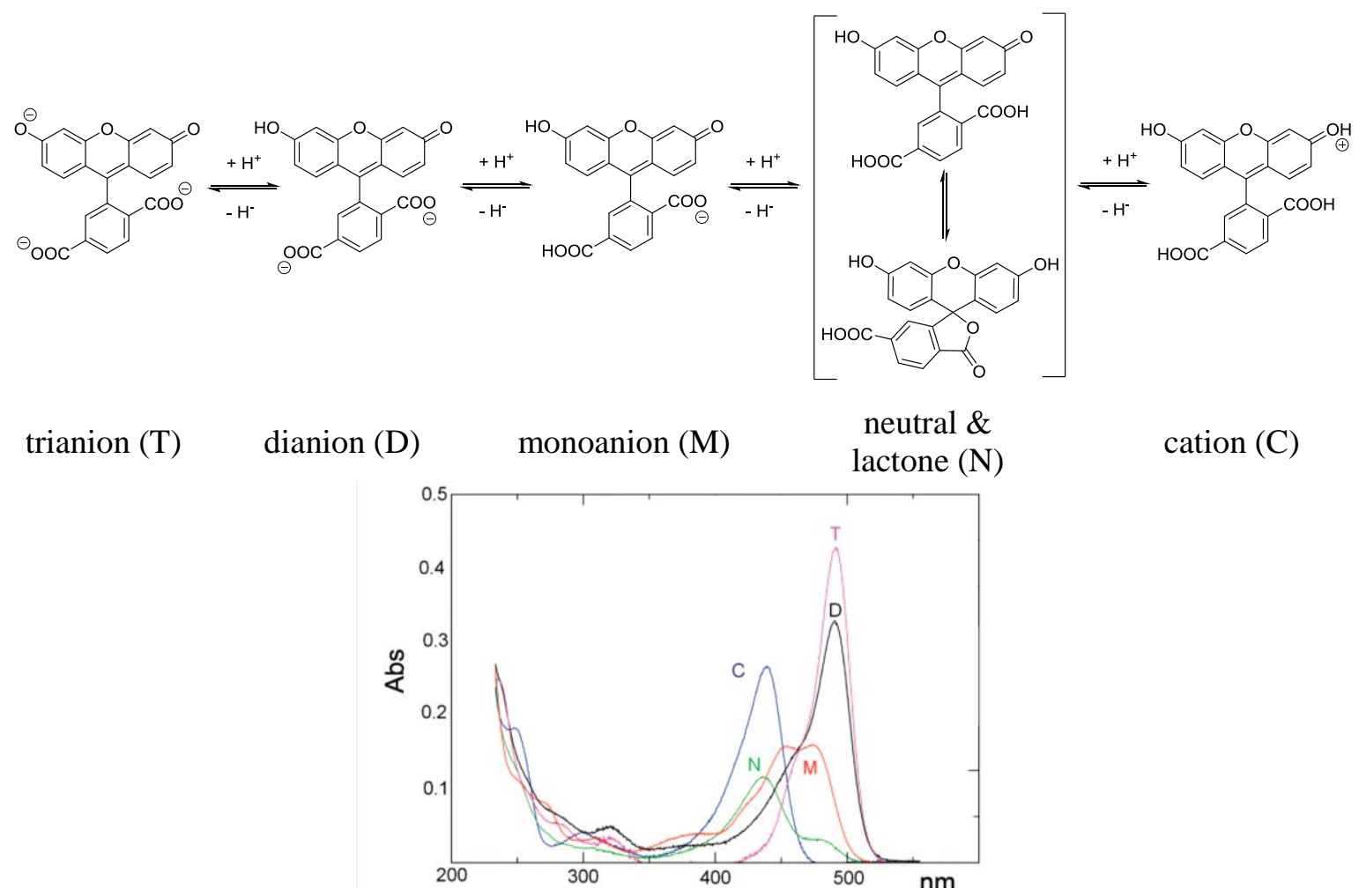

Figure 4.9 The change in chemical structure of (6)-carboxyfluorescein dependence on the $\mathrm{pH}$. With change in the protonation state the UV/Vis spectrum change of the molecule. ${ }^{[216]}$ Adapted with permission from M. Aschi et al, J. Org. Chem. 2008, 73, 3411-3417. Copyright (2008) American Chemical Society.

The fluorescence properties of fluorescein and its derivatives are well studied and show also a $\mathrm{pH}$ dependency in the spectroscopic properties, such as the fluorescence spectrum, the quantum yield and the molar attenuation coefficient. ${ }^{[217-219]}$ In Figure $4.10 \mathrm{a} \mathrm{pH}$ dependent fluorescence intensity of free carboxyfluorescein is shown. ${ }^{[220]}$

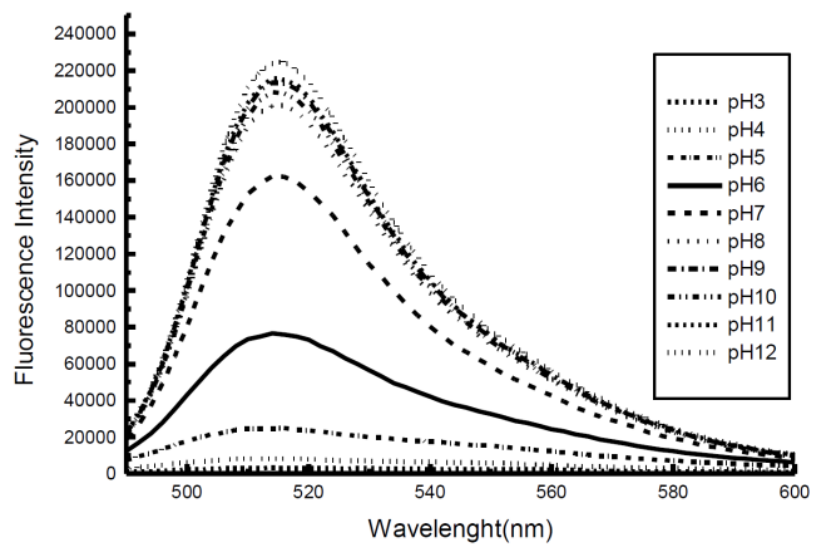

Figure 4.10 Emission spectra of carboxyfluorescein excited at $480 \mathrm{~nm}$ with different $\mathrm{pH}$ values. ${ }^{[220]}$ Adapted with permission from A. L. F. Baptista et al., J. Liposome Res. 2003, 13, 123-130. Copyright (2003) Taylor \& Francis. For free carboxyfluorescein the $\mathrm{p} K_{\mathrm{a}}$ is $6.36 .{ }^{[221]}$

In the laboratory practice when carboxyfluorescein is used as a dye, a ratio of two excitation wavelengths at one emission wavelength is used, instead of the total fluorescence intensity. ${ }^{[222-224]}$ This prevents effects such as photobleaching or variations in 
the excitation light intensity. ${ }^{[222]}$ It is possible to quench the fluorescence of fluorescein and its derivates by self-quenching with increased concentration ${ }^{[225]}$ or by quenching partners such as oxygen and iodine ions. ${ }^{[219,226]}$ For the above reasons, carboxyfluorescein and some of the derivates are ideal sensors for cell and near-membrane $\mathrm{pH}$ measurements.

\subsubsection{Considerations, synthesis and measurements}

\section{Considerations}

The work of KRAAYENHOF et al. showed a connection between the distance to a membrane surface of a $\mathrm{pH}$ active fluorophore and the $\mathrm{H}^{+}$concentration. ${ }^{[29]}$ For this study, systems with some degree of freedom between the alkyl chain anchoring and the fluorophore were used. The intention of the following work was to use a system, which is stable in the structure, easy to modify and that shows a defined distance to the membrane surface. Due to their rigid structure compared with $\alpha$-peptides (see Chapter 2.1), the $\beta$-peptides were tested as a potential lead structure. $\beta$-Peptides allow a predictable design, which is a prerequisite for the use and the establishment as a peptide based molecular ruler (see Figure 4.11). Additionally, the peptide structure enables a variety of modifications ranging from SPPS common condensation reactions to cross-coupling to HUISGEN cycloaddition. ${ }^{[112,117,118,227]}$

The $\beta$-peptide unit developed by RosT et al. was used as a starting point for the transmembrane anchoring of molecular sensors. ${ }^{[11,38]}$ This structure consists of 19 $\mathrm{D}-\beta^{3}$-homovalines $\left(\mathrm{D}-\beta^{3}\right.$-hVal) in the hydrophobic core flanked on each side by two D- $\beta^{3}$-homotryptophans (D- $\beta^{3}$-hTrp) and two D- $\beta^{3}$-homolysines (D- $\beta^{3}$-hLys) (see Figure 4.13). For the exact localisation and interaction of the $D-\beta^{3}-h \operatorname{Trp}$ and $D-\beta^{3}-h L y s$ residues with the membrane headgroup region, measurements like NMR or crystal structure are important, but such measurements are not yet available, whereby the position of an attached fluorophore relative to the membrane surface is not possible. Therefore, the first design was focused on two peptides, which differ in the number of amino acids in the hydrophilic peptide part. To detect clear differences in the following measurements, a mismatch in the hydrophilic region of the synthesised $\beta$-peptides of six amino acids was chosen. For an appropriate extension of the peptide for measurements at different distances to the membrane surface, some criteria have to be fulfilled. This extension should not exhibit adverse effects on the secondary structure of the transmembrane region and should have a high amount of water soluble groups. Furthermore, the fluorophore should be easily applied to the extension. On the basis of these assumptions, a system of an alternating 
sequence of $\mathrm{D}-\beta^{3}-\mathrm{hVal}$ and $\mathrm{D}-\beta^{3}-\mathrm{hLys}$ was tested. Due to the branching, $\beta^{3}-\mathrm{hVal}$ stabilises the helix and the $\beta^{3}$-hLys increases the water solubility. ${ }^{[83,88,89]}$ The extension by six amino acids of the existing transmembrane $\beta$-peptide motif, in consideration of a tilt angle of $16^{\circ}$ (in DOPC), a residue per turn of 3.0 and a rise per residue of $1.56 \AA$, lead to a height difference of $\sim 9 \AA$ (see Figure 4.11). ${ }^{[10,38]}$ Unpublished results of WEGNER and HALBMAIR regarding a residue per turn of 3.25 for the used system, were not considered. ${ }^{[228]}$

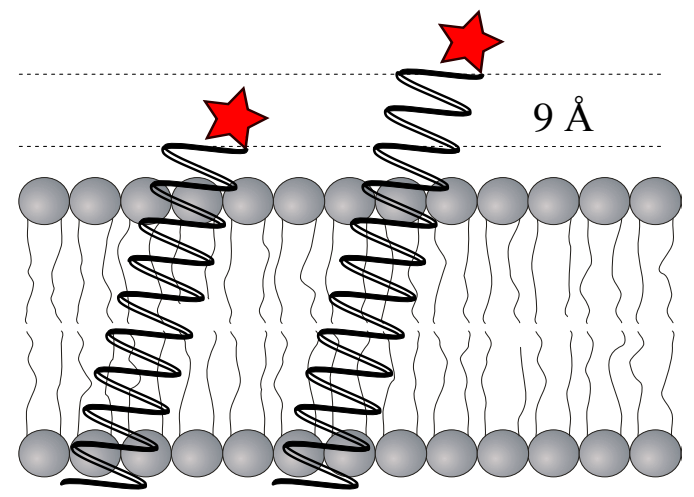

Figure 4.11 Simplified peptide model systems for near-membrane $\mathrm{pH}$ measurements.

The calculated $\sim 9 \AA$ difference is comparable with the height difference of U-2 and U-10 from the study of KRAAYENHOF et al. (8 $\AA) .{ }^{[29]}$ In contrast to KRAAYENHOF et al. carboxyfluorescein was used as a $\mathrm{pH}$ active functionalisation of the described $\beta$-peptides. Carboxyfluorescein is able to be attached by the carboxylic acid in position (5) or (6) during the SPPS. ${ }^{[22]}$ Thus, two artificial model systems for near-membrane $\mathrm{pH}$ measurements are designed and investigated in this study.

\section{Synthesis and structure results}

The structural properties and solubility of an alternating sequence of $\mathrm{D}-\beta^{3}-\mathrm{hVal}$ and D- $\beta^{3}$-hLys were tested with the synthesis of Pep 12. This $\beta$-peptide consists of $4 \times$ D- $\beta^{3}$ hVal and $5 \times \mathrm{D}-\beta^{3}$-hLys and should show, assuming a stable 14-helix, a 2 2/3 twist.

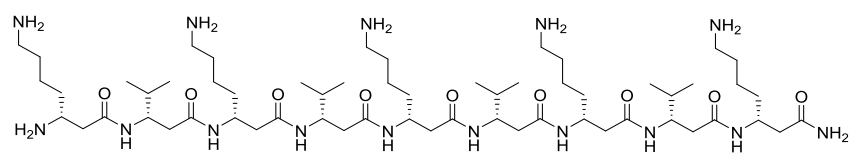

Pep 12

Figure 4.12 Chemical structure of Pep 12, with an alternating sequence of D- $\beta^{3}-h$ Val and D- $\beta^{3}-h L y s$.

The peptide Pep 12 was analysed by CD spectroscopy in ultrapure water $\left(0.6 \mathrm{M}, T=25^{\circ} \mathrm{C}\right.$, $d=0.01 \mathrm{~mm}$ ). Pep 12 shows a minimum at $196 \mathrm{~nm}$ and a maximum at $216 \mathrm{~nm}$ in the CD spectrum (see Appendix C). These values and the course of the curve correspond to a 14-helix. ${ }^{[62,70,76]}$ Compared with the transmembrane peptides, the CD spectrum shows a red 
shift. This is probably due to the peptide environment, despite the high concentration probable no aggregation of the helix bundles is observed (see Chapter 3.2). ${ }^{[76]}$

On this basis, the two peptides Pep 13 and Pep 14 were developed. These two peptides consist of a transmembrane unit, a linker and a fluorophore. Pep 14 has in contrast to Pep 13 an extension of six amino acids, which is based on Pep 12. The linker $\beta$-alanine is incorporated in the peptide structures to prevent an interaction of the fluorophore with the adjacent side chains, which could lead to a change in the secondary structure. Furthermore, $\beta$-alanine was used to obtain the smallest possible number of degrees of freedom by the $\mathrm{N}$-terminal attachment of the carboxyfluorescein. For example, if the fluorophore is bound to a lysine side chain, the distance between the membrane surface and the fluorophore is less determinable.

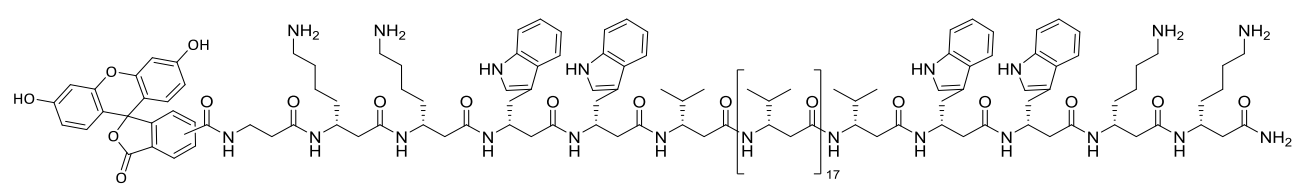

Pep 13

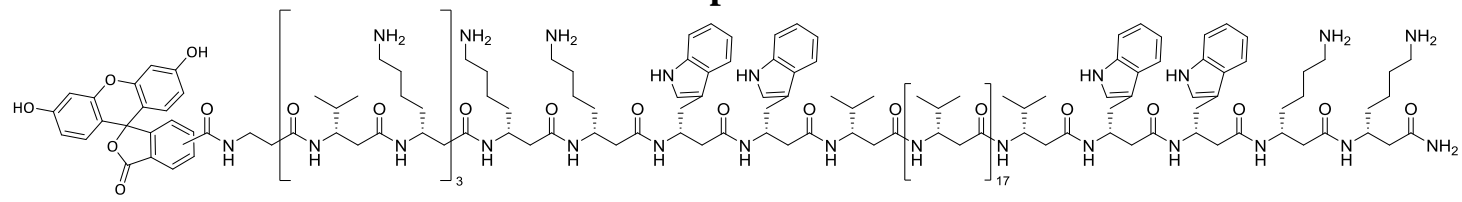

Pep 14

Figure 4.13 Chemical structure of Pep 13 and Pep 14.

The $\mathrm{pH}$ sensitive fluorophore carboxyfluorescein was bound to the peptide via an amide bond. At first, the pH active 5(6)-Carboxyfluorescein (5.00 eq) as well as PyBOP (4.70 eq) was solved in DMF (0.37 M) and DIPEA (10.00 eq) was added to the solution. The dry resin was mixed with the solution and shaken overnight. Due to the poor reproducibility of the same resin type, though with different swelling properties, other labelling methods were tested. Additionally, successful labelling was performed by three microwave-supported methods:

a) DIC (10.00 eq)/Oxyma (5.00 eq)/carboxyfluorescein (5.00 eq) double coupling $\left(25 \mathrm{~W}, 5 \mathrm{~min}, 90^{\circ} \mathrm{C}\right)$,

b) HATU (4.90 eq)/HOAt (5.00 eq)/DIPEA (10.00 eq)/carboxyfluorescein (5.00 eq), double coupling $\left(25 \mathrm{~W}, 15 \mathrm{~min}, 75^{\circ} \mathrm{C}\right)$,

c) PyAOP (5.00 eq)/HOAt (5.00 eq)/DIPEA (10.00 eq)/carboxyfluorescein (5.00 eq), double coupling $\left(25 \mathrm{~W}, 60 \mathrm{~min}, 75^{\circ} \mathrm{C}\right)$. 
After the successful labelling the washed resin was treated with a solution of $\mathrm{NMP} /$ piperidine $(v / v ; 80: 20)$ for $15 \mathrm{~min}$ at RT to remove the phenolic ester. ${ }^{\text {[230] }}$

In addition to carboxyfluorescein-labelled peptides, a derivative of Pep 14 was synthesised with the fluorophore 7-nitrobenz-2-oxa-1,3-diazol-4-yl (NBD) (Pep 15, see Figure 4.14). This peptide enables the distribution of incorporated $\beta$-peptides with a hydrophilic extension in membranes by the established NBD reduction method. ${ }^{[231]}$

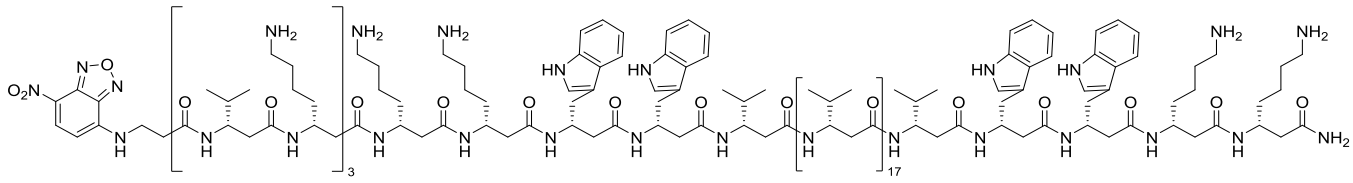

\section{Pep 15}

Figure 4.14 Chemical structure of Pep 15.

Therefore, the fluorophore was reduced by the non-permeating sodium dithionite $\left(\mathrm{Na}_{2} \mathrm{~S}_{2} \mathrm{O}_{4}\right)$, what results in the loss of fluorescence depending on the accessibility to the added $\mathrm{Na}_{2} \mathrm{~S}_{2} \mathrm{O}_{4} \cdot{ }^{[231]}$ The peptide labelling was performed with NBD-F (5.00 eq) and DIPEA (20.00 eq) in DMF. This solution was given to the dry resin for gentle agitation overnight.

\section{Measurements of Pep 13 - Pep 15}

To determine the properties of the peptides described above, different methods were used. First, the distribution of the peptide Pep 15 in the membrane was investigated by NBD reduction in DOPC vesicles. In this method, $\mathrm{Na}_{2} \mathrm{~S}_{2} \mathrm{O}_{4}$ was added to a vesicle solution containing a NBD-labelled peptide. The decomposition of $\mathrm{S}_{2} \mathrm{O}_{4}{ }^{2-}$ into radical $\mathrm{SO}_{2}{ }^{--}$in an aqueous solution leads to a reduction of NBD to 7-amine-2,1,3-benzoxadiazol-4-yl (ABD). ${ }^{[231]}$ The impermeability of the membranes for these ions results in a selective reduction of the peptides, orientated with NBD on the outside of the vesicle. The degree of reduction can be observed by fluorescence spectroscopy, as ABD shows no fluorescence in contrast to NBD. Consequently, LUV were produced with a P/L ratio of 1:100. The rehydration of the prepared lipid films and the extrusion took place in a phosphate buffer $\left(10 \mathrm{mM} \mathrm{Na}_{2} \mathrm{HPO}_{4}, 150 \mathrm{mM} \mathrm{NaCl}, \mathrm{pH} 7.6\right) .40 \mu \mathrm{L}$ of the vesicle suspension was diluted in $1960 \mu \mathrm{L}$ buffer $(0.20 \mathrm{mM}$ lipid in cuvette). After the stabilisation of the fluorescence, $20 \mu \mathrm{L}$ of a freshly prepared $50 \mathrm{mM}$ sodium dithionite solution in ultrapure water were added to the lipid solution. When no major changes in fluorescence could be observed, a $10 \%$ solution of Triton X-100 in ultrapure water was added, which leads to the destruction of the vesicle. From this measurement, an approximate distribution of Pep 15 was 
observed with $50 \%$ in the outer leaflet and $50 \%$ in the inner leaflet of the vesicle (see Figure 4.15).

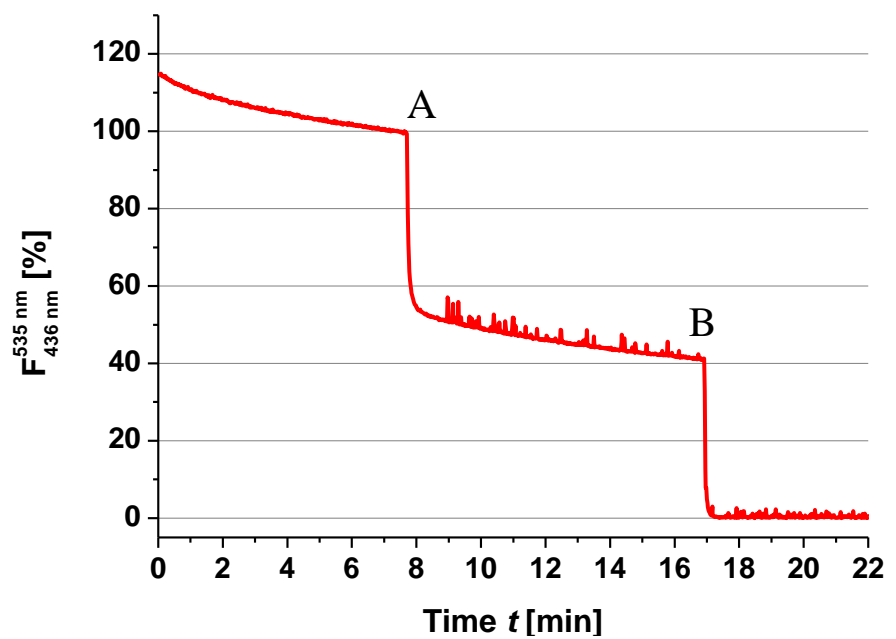

Figure 4.15 Detection of the peptide distribution in DOPC vesicles by NBD emission $\left(\lambda_{\mathrm{Ex}}=436 \mathrm{~nm}, \lambda_{\mathrm{Em}}=535 \mathrm{~nm}\right)$. Therefore, Pep 15 was incorporated in DOPC LUVs $(\sim 100 \mathrm{~nm}$, P/L ratio $=1: 100$, $\left.T=25{ }^{\circ} \mathrm{C}, \mathrm{rpm}=500\right)$. $\mathrm{A}=$ addition of $\mathrm{Na}_{2} \mathrm{~S}_{2} \mathrm{O}_{4} ; \mathrm{B}=$ addition of Triton X-100.

This distribution was obtained under the premise of an equal bleaching effect of the inside and outside fluorophores by excitation. Such a bleaching effect was observed from the beginning of the measurement, over the addition of $\mathrm{Na}_{2} \mathrm{~S}_{2} \mathrm{O}_{4}(\mathrm{~A}$ in Figure 4.15) and until the addition of Triton X-100 (B in Figure 4.15). After the distribution of the used peptides in the membrane was known, the properties of the peptide-bound carboxyfluorescein were investigated. Therefore, the concentration dependent absorption behaviour of peptide Pep 14 in $\mathrm{MeOH}$ was examined. It is known from the literature that free carboxyfluorescein shows self-quenching effects above a certain concentration. ${ }^{[225]}$ An increased carboxyfluorescein concentration lead to a dimerisation. This dimerisation leads to the loss of the fluorescence and can be observed by change in the UV/Vis absorption spectrum. In order to examine the self-quenching effect of the peptide-bound carboxyfluorescein, Pep 14 was dissolved in various concentrations in $\mathrm{MeOH}$ (see Figure 4.16). $\mathrm{MeOH}$ was chosen because the high hydrophobic transmembrane moiety shows a good solubility in organic solvents $(\mathrm{MeOH}, \mathrm{TFE})$. Based on $\mathrm{CD}$ measurements ${ }^{[94]}$ in organic solvents (additionally unpublished results of $\mathrm{WEGNER}^{[15]}$ ), non or less aggregation was presumed (Chapter 3.2.1). It turned out that the carboxyfluorescein-labelled peptides also show a concentration-dependent change in their absorption behaviour. At low peptide concentrations, the spectra show a maximum at $500 \mathrm{~nm}$. At higher concentrations the absorption maxima shift to smaller wavelengths and another maximum appears. These two absorption maxima were at $457 \mathrm{~nm}$ and $484 \mathrm{~nm}$. Although, the 3-dimensional 
consideration of Pep 14 solved in $\mathrm{MeOH}$ presented here is not directly transferable to a 2-dimensional peptide behaviour in lipid membranes, yet this measurement indicates a reference point and shows a concentration dependency in the absorption of Pep 14, what could result in a variation of the fluorescence.

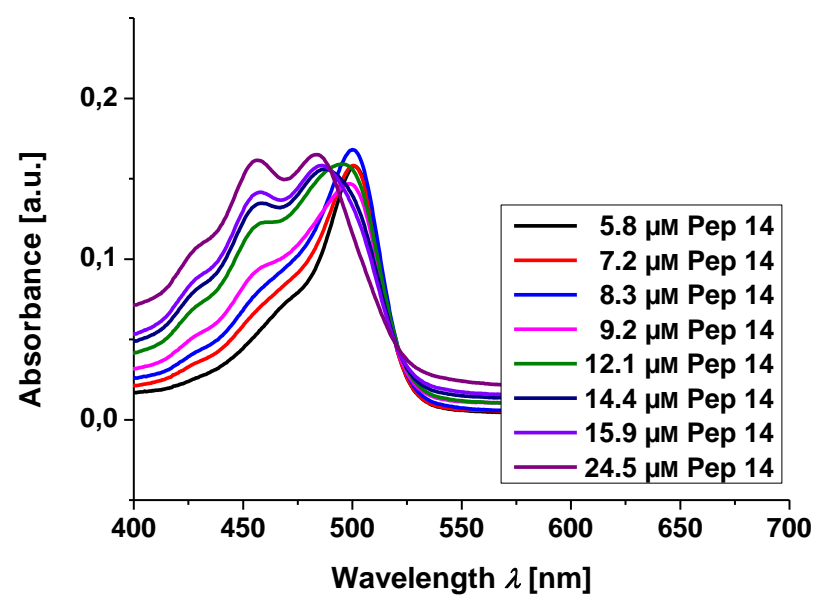

Figure 4.16 UV/Vis absorption spectra of Pep 14 in $\mathrm{MeOH}$ with variation of the peptide concentration $\left(\mathrm{T}=25{ }^{\circ} \mathrm{C}\right)$.

In the following experiments, the properties of the carboxyfluorescein-labelled peptides Pep 13 and Pep 14 incorporated in model membranes were investigated. It is known from KRAAYENHOF et al. that the behaviour of $\mathrm{pH}$ sensitive molecules in proximity to membranes could be affected by the lipid composition. ${ }^{[29]}$ One of the methods used in this study is an acid/base titration. For this titration it is assumed that the membrane surface interacts with ions due to the surface charge. A negatively charged membrane surface attracts positive ions like $\mathrm{H}^{+}$from the bulk solution. ${ }^{[232,233]}$ This results in a difference in the bulk solution $\mathrm{pH}$ and the membrane surface $\mathrm{pH}$. In the titration, the varying development of both these $\mathrm{pH}$ values is measured. Therefore, the $\mathrm{pH}$ of the bulk solution is measured by a $\mathrm{pH}$ meter and the surface $\mathrm{pH}$ by a membrane-bound sensor or the amount of standard solution is used in conjunction with the point of change of a membrane-bound sensor. ${ }^{[29,31]}$ Similar titrations were carried out with proteins, whether membrane-bound or water soluble proteins. ${ }^{[32,221,234]}$ Increasing the negative surface charge by charged headgroups can lead to an increasing affinity of $\mathrm{H}^{+}$on the surface (see Figure 4.17). In the study of SANDÉN et al., a difference was shown between dissolved fluorophore (fluorescein and Oregon green) compared to membrane-bound fluorophore. None or marginal difference in $\mathrm{p} K_{\mathrm{a}}$ was observed at DOPC and DOPG vesicles. ${ }^{[31]}$ However, KRAAYENHOF et al. observed by titration a change in the $\mathrm{p} K_{\mathrm{a}}$ for different molecules and lipid composition (see Figure 4.7 and Table 4.2). 


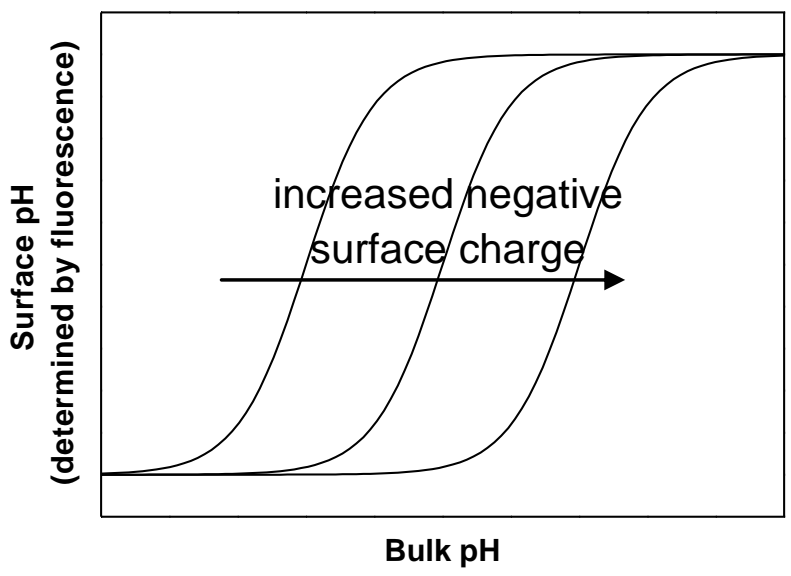

Figure 4.17 Schematic representation of $\mathrm{p} K_{\mathrm{a}}$ shifts by consideration of the bulk $\mathrm{pH}$ and the surface $\mathrm{pH}$ (determined by Fl ratio) depending on the surface charge of the membrane.

Due to the influence of the buffer to the membrane-bound fluorophores, the first step was to work with an elevated buffer concentration above the limit of $10 \mathrm{~mm}$ presumed by GENNIS. ${ }^{[208]}$ For this, the peptides were incorporated in $0.38 \mu \mathrm{mol} \mathrm{LUV} \mathrm{(P/L} \mathrm{ratio} \mathrm{of}$ 1:200) by the extruder method consisting of POPC, DOPC or DOPC/DOPG (50:50) with a phosphate-buffered saline (PBS) buffer $\left(137 \mathrm{mM} \mathrm{NaCl}, 2.7 \mathrm{mM} \mathrm{KCl}, 10 \mathrm{mM} \mathrm{Na} 2 \mathrm{HPO}_{4}\right.$, $1.8 \mathrm{mM} \mathrm{KH}_{2} \mathrm{PO}_{4}, \mathrm{pH}$ 7.4). Subsequently, for the measurement the vesicles were diluted to a total concentration of $19.0 \mathrm{nM}$ lipids in buffer ( $2 \mathrm{~mL}$ total volume), partly mixed with $0.95 \mu \mathrm{L}$ carbonylcyanid-m-chlorphenylhydrazon (CCCP) of a $0.2 \mathrm{mM}$ solution in DMSO and titrated with $2-3 \mathrm{M} \mathrm{NaOH}$ and $\mathrm{HCl}$ solutions. For the measurement, the sample was first acidified and then basified, started from a $\mathrm{pH}$ of 7.4. Preliminary examinations of $\mathrm{pH}$ titration with and without $\mathrm{CCCP}$ has shown unusual fluorescence behaviour in a $\mathrm{pH}$ range greater than 8 (Example in Figure 4.18 a). When stimulated with one wavelength $\left(F_{490}^{520}\right)$ some probes show a small decrease in the fluorescence. This effect can be eliminated by considering the ratio of two extinguish wavelength at one emission wavelength $\left(F_{490}^{520} / F_{450}^{520}\right.$; see Figure 4.18 b). ${ }^{[223]}$ In addition, the ratiometric consideration of the fluorescence enabled the influence of negative effects such as photobleaching. 
a)

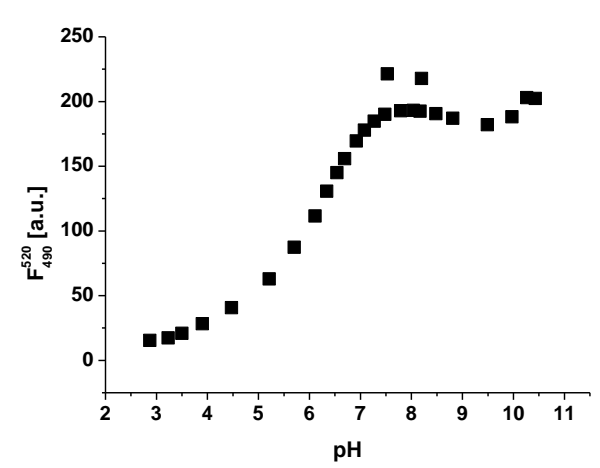

b)

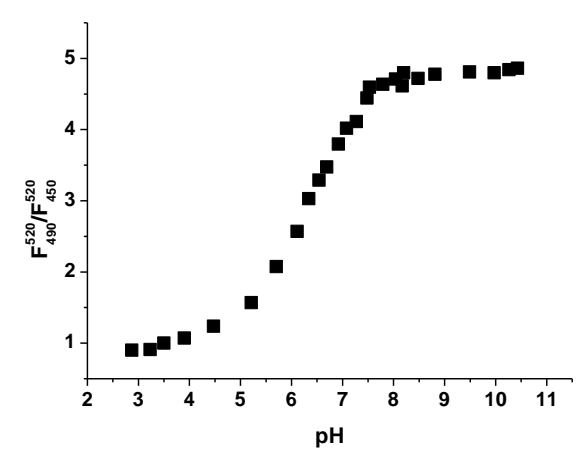

Figure 4.18 Titration of Pep 14 in DOPC vesicle $\left(\mathrm{P} / \mathrm{L}\right.$ ratio $=1: 200, T=25^{\circ} \mathrm{C}, \mathrm{rpm}=500$ ) in PBS buffer (probe without $\mathrm{CCCP).} \mathrm{a)} \mathrm{Measurement} \mathrm{of} \mathrm{one} \mathrm{observed} \mathrm{wavelength} \mathrm{and} \mathrm{b)} \mathrm{ratio} \mathrm{of} \mathrm{two} \mathrm{excitation} \mathrm{wavelength} \mathrm{at} \mathrm{one}$ emission wavelength $F_{490}^{520} / F_{450}^{520}$.

Additionally, a difference in the ratiometric fluorescence $F_{490}^{520} / F_{450}^{520}$ was detected from the acidified step to the basified step of peptides which were used after HPLC purification (HPLC solvents $+0.1 \%$ TFA) and lyophilisation. Figure 4.19 shows a sample in which the peptide was additionally treated with an excess of TFA and subsequently lyophilised to increase the effect to better visualise. It appeared for $F_{490}^{520} / F_{450}^{520}$ a maximum ratio of $\sim 10$ at the beginning of the measurement. The following acidified step leads to a fluorescence ratio of $\sim 1$ followed by a $F_{490}^{520} / F_{450}^{520}$ maximum in the basified step of $\sim 4$.

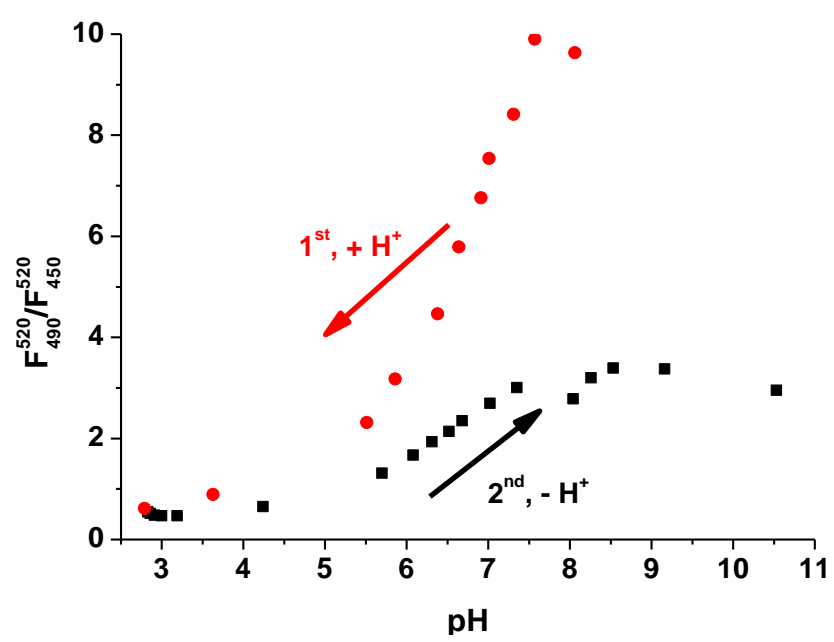

Figure 4.19 Titration of Pep 14 in POPC vesicle $\left(\mathrm{P} / \mathrm{L}\right.$ ratio $=1: 200, \mathrm{~T}=25^{\circ} \mathrm{C}, \mathrm{rpm}=500$ ) in PBS buffer (probe with CCCP). In red the acidification step (performed $1^{\text {st }}$ ) and in black the basification step (performed $2^{\text {nd }}$,) is presented. There is a difference in fluorescence ratio at pH 5.5 and higher for the two series of measurements.

This difference in the fluorescence ratio maximum can be observed due to formation of TFA salts with the fluorophore or the lysine side chains and can be prevented by an additional lyophilisation of the peptides form $\mathrm{H}_{2} \mathrm{O}$ or dioxane with a small amount of a $1 \mathrm{M}$ $\mathrm{HCl}$ solution. 
The titration of the peptides Pep 13 and Pep 14 was carried out in a PBS buffer (137 mM $\left.\mathrm{NaCl}, 2.7 \mathrm{mM} \mathrm{KCl}, 10 \mathrm{mM} \mathrm{Na} 2 \mathrm{HPO}_{4}, 1.8 \mathrm{mM} \mathrm{KH} \mathrm{KO}_{4}\right)$ and in a HEPES buffer $(10 \mathrm{mM}$ $\mathrm{KCl}, 2 \mathrm{mM} \mathrm{HEPES})$. These two measurements were performed to show the titration behaviour of the peptides above and below a buffer concentration of 10 mM. ${ }^{[208]}$ To break down a $\mathrm{H}^{+}$gradient between the vesicle inside and outside, carbonyl cyanide- $m$ chlorophenyl hydrazone (CCCP) dissolved in DMSO was added in a CCCP/lipid ration of 1:2010. The titration in a high concentrated buffer (PBS) was performed with DOPC vesicles or DOPC/DOPG (50:50) vesicles and a P/L ratio of 1:200. DOPG was incorporated into the vesicle as a negative charged lipid to observe $\mathrm{H}^{+} /$membrane effects, despite the high buffering concentrations which should prevent such an effect. The vesicles were obtained by the extrusion method. Therefore, the rehydrated lipid films $(0.38 \mu \mathrm{mol}$ lipid) were extruded through a $100 \mathrm{~nm}$ pore (size distribution in the Appendix C). Afterwards $1900 \mu \mathrm{L}$ buffer, $100 \mu \mathrm{L}$ vesicle suspensions and $0.95 \mu \mathrm{L}$ of a $0.2 \mathrm{mM} \mathrm{CCCP}$ solution in DMSO were mixed and titrated with an aqueous $2 \mathrm{M} \mathrm{NaOH}$ and $2 \mathrm{M} \mathrm{HCl}$ solution. The beginning of the titration was at the starting $\mathrm{pH}$ of the buffer $(\sim 7.4)$ and was performed first towards a low $\mathrm{pH}(\sim 3)$ followed by a titration to high $\mathrm{pH}(\sim 11)$. Each measurement was performed three times with different prepared probes (see Appendix C). As a sigmoidal fit curve of the fluorescence excitation ratio $F_{490}^{520} / F_{450}^{520}$ the BOLTZMANN fit was used. ${ }^{[235]}$ The $\mathrm{p} K_{\mathrm{a}}$ value of the measurements were determined as the inflection point and present in Table 4.1.

Table 4.1 $\mathrm{pK}_{\mathrm{a}}$ Values resulting from fluorescence titration of Pep 13 and Pep 14 in DOPC or DOPC/DOPG vesicles (PBS buffer, number of measurements $=3$ ).

\begin{tabular}{|c|cc|}
\hline & DOPC & DOPC/DOPG (50:50) \\
\hline Pep 13 & $6.33 \pm 0.04$ & $6.38 \pm 0.04$ \\
\hline Pep 14 & $6.18 \pm 0.05$ & $6.50 \pm 0.07$ \\
\hline
\end{tabular}

In different lipid composition the values show for Pep 13 no shift in the $\mathrm{p} K_{\mathrm{a}}$ in the magnitude of previous studies performed by KRAAYENHOF et al. ${ }^{[236]}$ They demonstrated different titration behaviours for the synthesised molecules (see Figure 4.7) depending on the distance to the membrane and lipid composition (Table 4.2). ${ }^{[236]}$ Pep 14 shows a slightly different $\mathrm{p} K_{\mathrm{a}}$ value for the two membrane systems in PBS buffer, the meaningfulness of these values is not known and must be confirmed by further studies and methods. 
Table 4.2 Apparent $\mathrm{p} K_{\mathrm{a}}$ values of fluorophores at various distances from the membrane and different PC and PA lipid ratio, determinate by fluorimetric titrations.

\begin{tabular}{|ccccccc|}
\hline & 0 \% PA & 2.5 \% PA & 5 \% PA & $\mathbf{7 . 5} \%$ PA & 10 \% PA & 20 \% PA \\
\hline U-2 & 8.89 & 9.32 & 9.61 & 9.80 & 10.10 & 10.35 \\
\hline U-6 & 7.08 & 7.42 & 7.67 & 7.79 & 7.91 & 8.08 \\
\hline U-10 & 8.55 & 8.85 & 9.03 & 9.23 & 9.28 & 9.32 \\
\hline U-13 & 7.57 & 7.83 & 7.95 & 8.10 & 8.16 & 8.27 \\
\hline
\end{tabular}

In order to investigate the repeatability of the process, the $\mathrm{pH}$ was changed ten times for the probes given in Table 4.1. At high $\mathrm{pH}$ values the fluorescence maximum $F_{490}^{520}$ shows for Pep 13 a maximum fluorescence intensity reduction of $16-35 \%$. However, this reduction has no influence on the $F_{490}^{520} / F_{450}^{520}$ ratio (see Appendix C) and a repeatability for all probes exists.

The titration with a buffer concentration < $10 \mathrm{mM}$ (HEPES) was performed, following KRAAYENHOF et al., with vesicles of DOPC or DOPC/DOPA (90:10) and a P/L ratio of 1:1000. ${ }^{[236]}$ The vesicles were obtained by the extrusion method with a filter pore size of $400 \mathrm{~nm}$. Therefore $500 \mu \mathrm{L}$ buffer was given to the dry lipid film and the rehydrated lipid films $(1.27 \mu \mathrm{mol}$ lipid) were extruded. Afterwards $1900 \mu \mathrm{L}$ buffer, $100 \mu \mathrm{L}$ vesicle suspensions and $3.16 \mu \mathrm{L}$ of a $0.2 \mathrm{mM}$ CCCP solution in DMSO were mixed and titrated with $2 \mathrm{M} \mathrm{NaOH}$ and $2 \mathrm{M} \mathrm{HCl}$ aqueous solution. The titration procedure was analogue to the titration in PBS buffer. The $\mathrm{p} K_{\mathrm{a}}$ values of these measurements are given in Table 4.3. These values show no shift in the $\mathrm{p} K_{\mathrm{a}}$, which is in the magnitude of the values given in Table 4.2.

Table 4.3 $\mathrm{pK}_{\mathrm{a}}$ Values resulting from fluorescence titration of Pep 13 and Pep 14 in DOPC or DOPC/DOPA vesicles (HEPES buffer, number of measurements $=3$ ).

\begin{tabular}{|ccc|}
\hline & DOPC & DOPC/DOPA (90:10) \\
\hline Pep 13 & $6.11 \pm 0.11$ & $6.08 \pm 0.04$ \\
\hline Pep 14 & $6.10 \pm 0.15$ & $6.43 \pm 0.14$ \\
\hline
\end{tabular}

Although the vesicle preparation was carried out analogously to the method described by KRAAYENHOF et al., the size distribution measurements of the vesicles by DLS, showed no homogenous size distribution (see Appendix C). These heterogeneous size distributions are independent of vesicle probes with peptides (see Appendix C) or without peptides (results not shown). In contrast to these measurements, the PBS buffer vesicle showed one size 
distribution (see Appendix C). It should also be noted that the measurements of Pep $\mathbf{1 4}$ in the lipid systems with negative charges DOPC/DOPG (50:50) or DOPC/DOPA (90:10) show the largest $\mathrm{p} K_{\mathrm{a}}$ shift in comparison to the other measured values. The measured values are in the range of dissolved carboxyfluorescein $\left(\mathrm{p} K_{\mathrm{a}}=6.36\right)^{[221]}$ with a $\mathrm{p} K_{\mathrm{a}}$ value of $\sim 6.5$ (see Figure 4.8). However, the meaningfulness of these values is not known and has to be confirmed by further studies.

\subsubsection{Outlook for Pep 13 and Pep 14}

In addition to the titrations, other methods are still in processes to examine the properties of the $\mathrm{pH}$ functionalised $\beta$-peptides, whereby a broad understanding of the mechanisms should be obtained. Due to the complexity of the measurements, they are not yet finished. However, the measurements and preliminary investigations will be discussed briefly. Various studies on labelled lipids were carried out in the WIDENGREN group by fluorescence correlation spectroscopy (FCS). ${ }^{[31-34]}$ In these studies, inter alia, lipids were labelled with $\mathrm{pH}$ active fluorophores and vesicles (small unilamellar vesicle, SUV) or lipid discs containing the labelled lipids were prepared. Subsequently, various $\mathrm{pH}$ values were set and the respective autocorrelation functions were recorded (see Figure 4.20). ${ }^{[34]}$ These measurements allow the observation of local $\mathrm{H}^{+}$exchange close to the membrane. By this method, $\mathrm{a} \mathrm{H}^{+}$-collecting effect of DOPG could be determined, whereby the cross section of fluorescein will be increased by 100 times. Furthermore, it could be shown that the DOPA lipid headgroups store $\mathrm{H}^{+}$close to the membrane, which are in a rapid equilibrium with a membrane-bound fluorophore. ${ }^{[34]}$ The varying of the surface charge and ion concentration could allow the determination of the surface potential and the distance of the fluorophores to the surface. ${ }^{[31,34]}$

a)

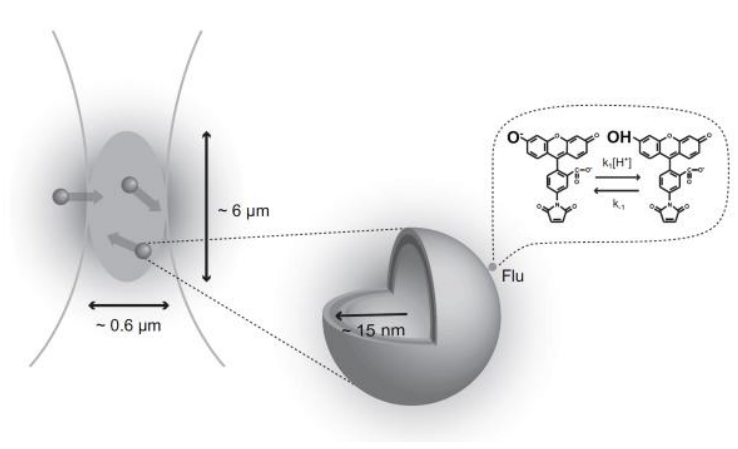

b)

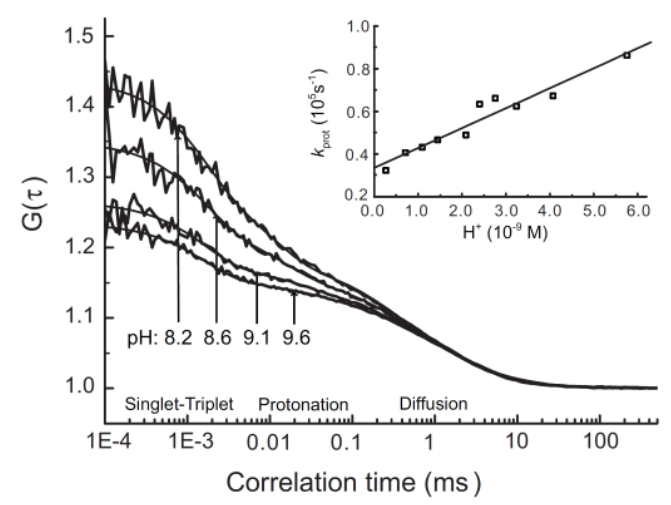

Figure 4.20 a) Confocal detection volume of FCS measurement for observation of fluorescence labelled SUV movement. b) Example FCS curves of fluorescein labelled vesicles at different pH values. ${ }^{[34]}$ Reprinted with permission from M. Brändén et al, Proc. Natl. Acad. Sci. 2006, 103, 19766-19770. Copyright (2006) National Academy of Sciences. 
The following FCS project will be done in cooperation with the ENDERLEIN group. To reproduce the results of the WIDENGREN group, vesicles were prepared according to the sonicated based method, which resulted in unequal size distribution with multiple size maxima (unpublished results). Due to this, vesicle preparation with DOPG lipids, carboxyfluorescein-labelled lipids (DHPE-CF) and monensin as $\mathrm{Na}^{+} / \mathrm{H}^{+}$ionophore were tested. Therefore, the extrusion method with different filter pore sizes $(30 \mathrm{~nm}, 50 \mathrm{~nm}$, $100 \mathrm{~nm})$ and different fluorophore/lipid ratios (1:500, 1:1000, 1:3000, 1:5000, 1:7000, 1:10000, 1:30000) were performed. The vesicles with a ratio of 1:7000 and 1:10000 prepared with a filter pore size of $30 \mathrm{~nm}$ (average vesicle size of $\sim 78 \mathrm{~nm}$ ) in PBS buffer showed constant size distribution and usable FCS measuring properties. A major problem in these measurements is the exclusion of $\mathrm{CO}_{2}$, which induced a $\mathrm{pH}$ change during the measurement because an unbuffered system was used $(0.15 \mathrm{M} \mathrm{NaCl})$ for the FCS measurements.

In addition to the FCS measurements, the determination of the distance from the fluorophores to the surface will be performed in cooperation with the ENDERLEIN group. By the metal-induced energy transfer (MIET) an energy transfer takes place between an excited fluorophore and the surface plasmon of a metal structure. ${ }^{[237-239]}$ So far, distances in the order of magnitude of the inner and outer leaflet of a membrane were observed with this method (yet unpublished results). In this project, MIET would be helpful for pointing out the tilt of the peptides in the membrane, without the usual X-ray or ATR-FTIR technique and to prove the transmembrane position of the peptides in the membrane.

These studies will be supported by measurements performed in the POHL group. ${ }^{[186,195,196,204]}$ Here, the peptides Pep 13 and Pep 14 will be investigated by a UV induced membrane $\mathrm{H}^{+}$gradient. For this purpose, the peptides and (6,7dimethoxycoumarin-4-yl)methyl (DMCM) diethyl phosphate are incorporated in a planar membrane. DMCM-diethyl phosphate releases a $\mathrm{H}^{+}$by irradiation with UV light. This $\mathrm{H}^{+}$ can move along the surface of the membrane (see Figure $4.21 \mathrm{c}$ ). ${ }^{[196,204]}$ At a distance of $100 \mu \mathrm{m}$ to the site of the $\mathrm{H}^{+}$release, the fluorescence of the $\mathrm{pH}$ active molecule is measured (see Figure 4.21 a). In the case of membrane-bound fluorescein, the decreasing fluorescence is based on increased $\mathrm{H}^{+}$concentration, which indicates a movement of $\mathrm{H}^{+}$ across the membrane (see Figure 4.21 a). 
a)
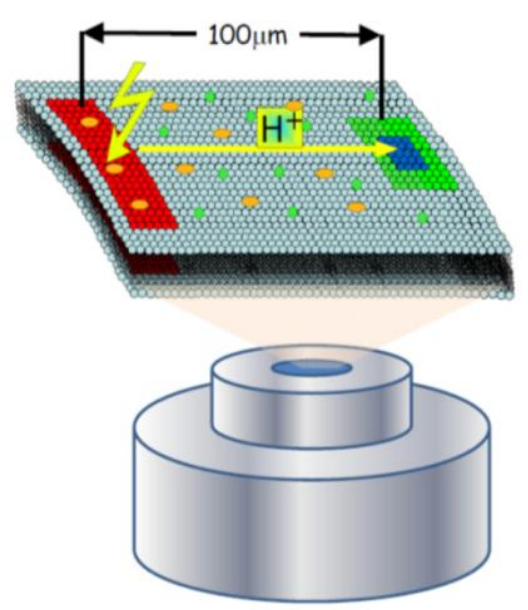

b)

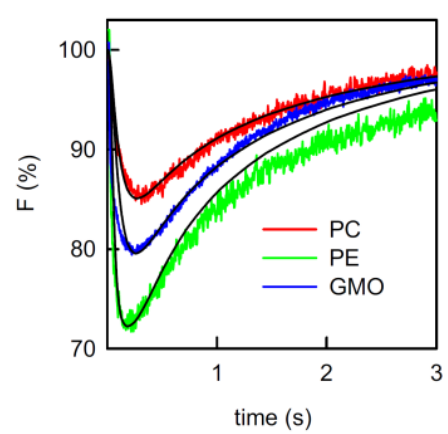

c)<smiles>CCOP(=O)(OC)OCc1cc(=O)oc2cc(OC)c(OC)cc12</smiles><smiles>CCOP(=O)(OC)O[C@H](O)C(=O)OC</smiles>

Figure 4.21 a) Measurement setup of the UV induced membrane $\mathrm{H}^{+}$gradient. ${ }^{[186]}$ The $\mathrm{H}^{+}$release takes place in the red area by UV-flash followed by $\mathrm{H}^{+}$along the membrane surface to the green/blue area. The horizontal planar lipid bilayer is observed by an inverse fluorescent microscope. Reprinted with permission from N. Agmon et al, Chem. Rev. 2016, 116, 7642-7672. Copyright (2016) American Chemical Society. b) Fluorescence degreases of membrane-bound $\mathrm{pH}$ active molecules by $\mathrm{H}^{+}$arrival in different lipid compositions. ${ }^{[204]}$ Reprinted with permission from A. Springer et al, Proc. Natl. Acad. Sci. 2011, 108, 14461-14466. Copyright (2011) Proceedings of the National Academy of Sciences. c) UV induced $\mathrm{H}^{+}$release by DMCM-diethyl phosphate. ${ }^{[186,204]}$

In the case of a successful incorporation of the peptides into the membrane, this method can show the $\mathrm{H}^{+}$migration across the membrane and the influences at different distances to the membrane surface.

\subsubsection{Conclusion and general outlook}

Cellular $\mathrm{pH}$ plays an important role in many processes in the cell. Especially for the energy production in cells, the near-membrane $\mathrm{H}^{+}$concentration is important. ${ }^{[1]}$ Appropriate probes for examining this concentration are the basis for understanding the mechanism of the vital $\mathrm{pH}$.

The described $\beta$-peptide derivates Pep 13 and Pep 14 showed the successful modification of $\beta$-peptides with carboxyfluorescein as $\mathrm{pH}$ active fluorophores. ${ }^{[33,221]}$ For this purpose, various coupling conditions were established. Pep 14 was elongated by using an alternating sequence of $\mathrm{D}-\beta^{3}-\mathrm{hVal}$ and $\mathrm{D}-\beta^{3}$-hLys attached to the transmembrane motif developed by ROST et al. ${ }^{[38]}$ Due to an increased lysine concentration, the water solubility of this elongation was improved. ${ }^{[88]}$ The distribution of the extended peptide was determined by Pep 15 and showed $\sim 50 \%$ in the outer leaflet and $~ 50 \%$ in the inner leaflet in LUV $(\sim 100 \mathrm{~nm})$. In order to confirm these results the peptides have to be observed in 
different membrane systems and different vesicle sizes. Measurements have also shown that the fluorophore properties, such as self-quenching, are preserved at the peptide-bound fluorophore and can be detected by UV/Vis spectroscopy. ${ }^{[25]}$ Titration of Pep 13 and Pep 14 was performed with a buffer concentration in a range of $<10 \mathrm{~mm}$ (HEPES) and $>10 \mathrm{mM}$ (PBS), which is postulated by GENNIS as a border for $\mathrm{H}^{+} / \mathrm{membrane}$ interaction. ${ }^{[208]}$ These titrations showed a $\mathrm{pH}$ dependence of the fluorescence for all performed measurements, which demonstrated the accessibility of the peptide-bound fluorophore to the surrounding $\mathrm{pH}$ changes. Therefore, the peptides were investigated in DOPC vesicles $(\sim 100 \mathrm{~nm}$ and $\sim 300 \mathrm{~nm})$ and in mixtures of DOPC/DOPG $(\sim 100 \mathrm{~nm}$, 50:50) or DOPC/DOPA ( 300 nm, 90:10). These various lipid compositions should influence the surface charge of the membrane, whereby the $\mathrm{H}^{+} /$membrane interaction can be changed. ${ }^{[29,31,34]}$ The evaluation of the titrations shows only a slight change in the $\mathrm{p} K_{\mathrm{a}}$ value in different lipid systems compared to the literature. ${ }^{[29]}$ In order to make an accurate statement, further measurements and methods have to be carried out to determine the influence of the membrane on the peptide-bound fluorophores.

For future peptide designs, the presented system can be modified in several ways, which allows the change of the peptide properties. One possible modification would be the change of the hydrophilic groups at the fluorophore bound side. Increased water solubility was achieved by a higher amount of $D-\beta^{3}$-hLys which can be protonated at the side chain amine group. This positive charged peptide could result in a repulsing effect of the $\mathrm{H}^{+}$and the peptide. This could be an indication why the measured $\mathrm{p} K_{\mathrm{a}}$ values of Pep $\mathbf{1 3}$ and Pep $14\left(\mathrm{p} K_{\mathrm{a}}=6.10-6.50\right)$ are in the range of dissolved carboxyfluorescein $\left(\mathrm{p} K_{\mathrm{a}}=6.36\right) .{ }^{[221]}$ Because of this it was attempted to synthesise two derivatives of Pep 14. Here, the hydrophilic D- $\beta^{3}$-hLys on the fluorophore-bound peptide side were replaced by D- $\beta^{3}$-homoserine $\left(\mathrm{D}-\beta^{3}\right.$-hSer) and $\mathrm{D}-\beta^{3}$-homoglutamine $\left(\mathrm{D}-\beta^{3}-\mathrm{hGln}\right)$, which are polar neutral amino acids. In previous studies performed by ABELE et al. $\beta$-peptides with $\beta^{3}$-hSer showed water solubility with a stabile secondary structure. ${ }^{[85]}$ In contrast, previous studies have shown an aggregation effect by transmembrane $\mathrm{D}-\beta^{3}-\mathrm{hGln} .{ }^{[94]}$ The feasibility of the synthesis of such modified peptides was tested. Therefore, Pep 16 and Pep 17 were synthesised by microwave-supported peptide synthesis following Method 2 (Chapter 2.5.2). For Pep 17 no successful synthesis was verified by mass spectrometry. For Pep 16 signals with a higher $\mathrm{m} / \mathrm{z}$ were observed, instead of the target mass. This additional mass of $n \times 96 \mathrm{~m} / \mathrm{z}$ was avoided by the treatment of the cleaved peptide with 
diluted $\mathrm{NaOH}$ solution and subsequent lyophilisation. The increased mass can be a result of the formation of a TFA ester with the $\mathrm{D}-\beta^{3}$-hSer side chain.

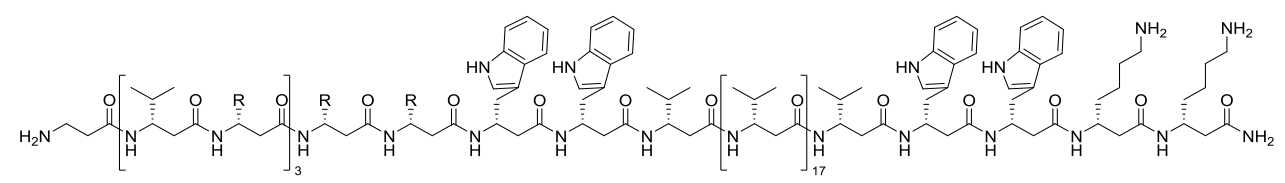

$$
\begin{aligned}
& \text { Pep } 16(\mathrm{R}=\mathrm{Ser}) \\
& \text { Pep } 17(\mathrm{R}=\mathrm{Gln})
\end{aligned}
$$

Figure 4.22 Chemical structure of Pep 16 and Pep 17.

However, a more difficult purification was observed because of the reduced polarity, compared with Pep 14. Nevertheless, this Pep 16 represents a neutral extension and requires further investigation. In addition to polar neutral amino acids, negative charged amino acids can be incorporated, which can lead to an attraction effect. Furthermore, as an alternative to the carboxyfluorescein used sensor, other $\mathrm{pH}$ active fluorophores can be investigated $^{[29,223]}$ or nitrile based $\mathrm{pH}$-sensors for IR measurements are possible. ${ }^{[240]}$ In summary, the synthesis and initial investigations represent a major potential for $\beta$-peptide-bound $\mathrm{pH}$-sensors due to their stability and variability. In this work, the first $\beta$-peptide based pH-sensors Pep 13 and Pep 14 have been developed, which could represent a starting point for this application.

\section{2 $\beta$-Peptide functionalisation with SPPS-compatible $\mathrm{Ca}^{2+}$-sensors}

\subsubsection{Intracellular $\mathrm{Ca}^{2+}$}

A variety of metal ions are important for maintaining key processes in life. ${ }^{[1,241]}$ The wide range of functions utilising metals, includes the osmotic balance and the electric activity of cells, the catalytic activity of enzymes as well as the stability and the activity of the DNA and RNA. ${ }^{[241]}$ Calcium, as one of the bulk elements, shows a wide field of application in the hard and the soft structures of organic systems. Calcium is with $1.9 \%$ the fifth common element in the human body after O, C, H and N. ${ }^{[242]}$ Furthermore, apart from strontium, calcium is the only metal that is found in the hard structures like skeleton and teeth. ${ }^{[241]}$ In soft structures, calcium mostly exists in its ionic form. Here, the function can be distinguished in the following categories: structural, electrical, catalytic cofactor and universal intercellular regulator functions. ${ }^{[241]}$ As an inorganic molecule, calcium ions $\left(\mathrm{Ca}^{2+}\right)$ are one of the most occurring second messengers besides the organic second messengers cyclic adenosine monophosphate (cAMP), inositol triphosphate $\left(\mathrm{IP}_{3}\right)$ and 
diacylglycerol (DAG). ${ }^{[243]}$ Among others, $\mathrm{Ca}^{2+}$ regulates the insemination of egg cells, ${ }^{[244,245]}$ the synaptic transmission ${ }^{[246-248]}$ as well as the apoptosis. ${ }^{[249,250]}$ Due to the concentration dependency of these applications, it is important to control the $\mathrm{Ca}^{2+}$ gradient in organic systems. ${ }^{[188]} \mathrm{The}^{\mathrm{Ca}^{2+}}$ concentration in the extracellular space is up to $2 \mathrm{mM}$. In contrast, the $\mathrm{Ca}^{2+}$ concentration in the intercellular space varies from $100 \mathrm{nM}$ in the cytoplasmic matrix up to $600 \mu \mathrm{M}$ in the endoplasmic reticulum. ${ }^{[188,251,252]}$ Especially in the transmission of nerve signals, the gradient of $\mathrm{Ca}^{2+}$ is an important regulatory element. ${ }^{\text {[253] }}$

During the stimulation of nerve cells, an incoming signal at the axon terminal opens voltage-dependent $\mathrm{Ca}^{2+}$ channels leading to a temporary increase of the presynaptic $\mathrm{Ca}^{2+}$ concentration. ${ }^{[246]}$ The increased $\mathrm{Ca}^{2+}$ concentration results in the activation of synaptotagmin (Syt), which works as an intercellular $\mathrm{Ca}^{2+}$-sensor. ${ }^{[254]}$ The basic structure of Syt consists of a transmembrane region and two $\mathrm{C} 2$ domains. In consequence of a mutation at the C2 domains, the 16 known Syt mutants (Syt1 - Syt 16) can be classified into $\mathrm{Ca}^{2+}$-binding and $\mathrm{Ca}^{2+}$-nonbinding proteins. ${ }^{[246]}$ For the SNARE mediated vesicle fusion only the $\mathrm{Ca}^{2+}$-binding proteins are of interest. Incoming $\mathrm{Ca}^{2+}$ bind to the $\mathrm{C} 2$ domains, which leads to a translocation of the Syt in the direction of the membrane due to the interaction of the protein-bound $\mathrm{Ca}^{2+}$ with the charged acidic lipid headgroups. ${ }^{[246,255]}$ Especially phosphatidylinositol phosphate (PI) lipids interact strongly with the Syt1/Ca ${ }^{2+}$ complex. These lipids are mostly found in the cytoplasmic membrane leaflet. ${ }^{[255,256]}$ In addition to the interaction with the lipids, an interaction of Syt and SNARE complex forming proteins takes place (see Figure 4.23). ${ }^{[246]}$

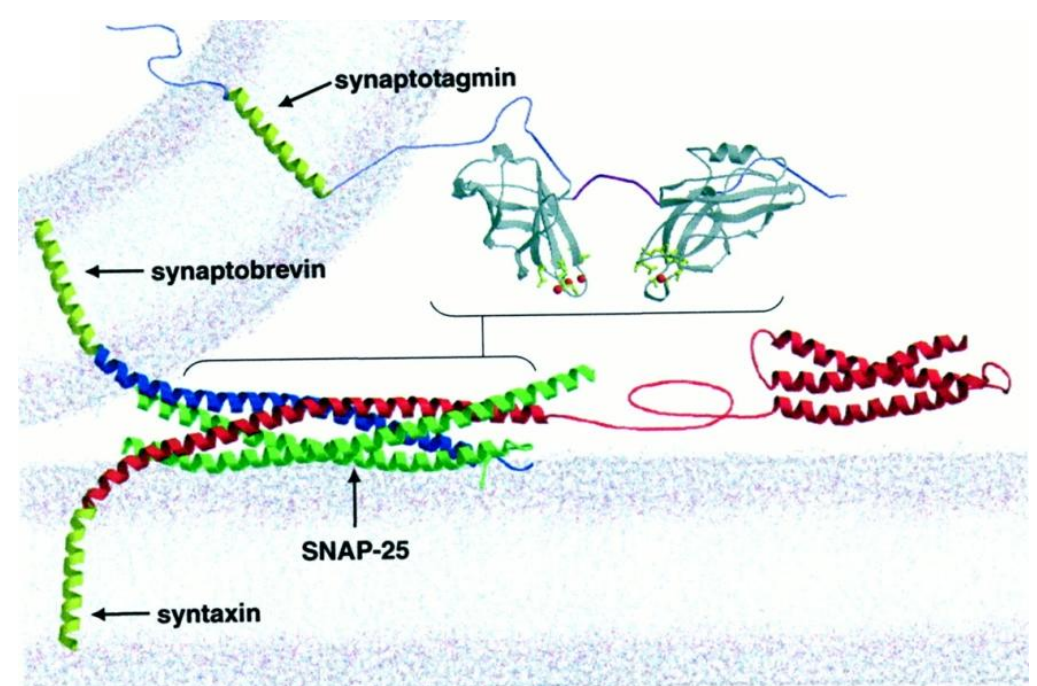

Figure 4.23 Modulated structure of the SNARE complex formed by synaptobrevin, SNAP-25, syntaxin and synaptotagmin. The parts connected with brackets interact with each other during the fusion process. ${ }^{[257]}$ Republished with permission of Journal of Neuroscience from "synaptotagmin Mutants Reveal Essential Functions for the C2B Domain in $\mathrm{Ca}^{2+}$-Triggered Fusion and Recycling of Synaptic Vesicles In Vivo", J. T. Littleton et al., 21 (5), 2001, 1421-1433 permission conveyed through Copyright Clearance Center, Inc. 
Moreover, studies without Syt have shown that $\mathrm{Ca}^{2+}$ also has a positive effect on vesicle fusion. ${ }^{[258]}$ Differences between SNARE and SNARE/Ca ${ }^{2+}$ induced fusion were observed in experiments with target SNAREs (t-SNAREs) and vesicle SNAREs (v-SNAREs) incorporated vesicles. ${ }^{[258]}$ Without prior $\mathrm{Ca}^{2+}$ addition a mixture of $\mathrm{t}$ - and v-SNARE vesicles lead to a strong increase in light scattering signal. In contrast, the same experiment with prior $\mathrm{Ca}^{2+}$ addition to one sort of vesicle produced a 4-fold weaker light scattering signal. ${ }^{[258]}$ The different light scattering signal intensity can be explained by two different vesicle processes. The omission of $\mathrm{Ca}^{2+}$ leads to the aggregation of both vesicle sorts. This aggregation without fusion leads to vesicle clusters with a much bigger diameter then vesicles after fusion. As a competitive process, the membrane fusion is triggered by a prior addition of $\mathrm{Ca}^{2+}$ which leads to ionic interaction of the membrane surfaces and the $\mathrm{Ca}^{2+}$. This results in smaller particle size compared to the vesicle clusters and shows the important function of $\mathrm{Ca}^{2+}$ in processes including biological membranes. ${ }^{[258]}$

\subsubsection{Interaction of $\mathrm{Ca}^{2+}$ with the membrane surface}

The interaction of the membrane surface with ions can be influenced by various factors. Depending on the composition of the membrane and the kind of cations, different interactions can occur. Monovalent alkali cations show only an adsorption effect on the membrane when negative charged lipids are incorporated. ${ }^{[259]}$ Divalent ions, like $\mathrm{Ca}^{2+}$, are adsorbed at the membrane surface even in the absence of negatively charged lipids. ${ }^{[188]}$ In general, the extracellular leaflet of membranes consists of neutral or zwitterionc components (phosphatidylcholine, sphingomylein, cholesterol). By the coordination of cations on the neutral surface a positive surface charge is created. ${ }^{[260-263]}$ This interaction of $\mathrm{Ca}^{2+}$ with the headgroups has a great influence on the properties of the lipid bilayer like the rigidity of lipids and their order. ${ }^{[188]}$ In this context, following effects were reported: conformational change of the lipids head region, ordering of acyl chains and lipid dehydration. ${ }^{[188]}$ Due to the important influence of $\mathrm{Ca}^{2+}$ in the membrane fusion, the interaction of the inner leaflet and $\mathrm{Ca}^{2+}$ is a major part of research for the transmission of signals. To imitate neuronal vesicles, a simple model system of $80 \%$ phosphatidylcholine (PC) and $20 \%$ phosphatidylserine (PS) is used. ${ }^{[188]}$ In a mixed membrane consisting of PC and PS, three different binding sites are available in the headgroup region. ${ }^{[188]}$ The carbonyl and phosphate groups of PC and PS bind $\mathrm{Ca}^{2+}$ as well as the carboxyl group of the PS headgroup. Using calculations it was tried to clarify the exact percentage of each possible binding partner. ${ }^{[188]}$ Figure 4.24 shows the calculated values for two different 
concentrations of $\mathrm{Ca}^{2+}$ and two different lipid compositions. The average number of $\mathrm{Ca}^{2+}$ in the first coordination sphere of the respective functional group is proportional to the thickness of the red lines. ${ }^{[188]}$ With increased $\mathrm{Ca}^{2+}$ concentration, the number of lipids per $\mathrm{Ca}^{2+}$ decreases. ${ }^{[188]}$ In addition, the binding of a PS carboxyl group to $\mathrm{Ca}^{2+}$ leads to a reorientation of the PS headgroup from the water interface to the membrane phase.

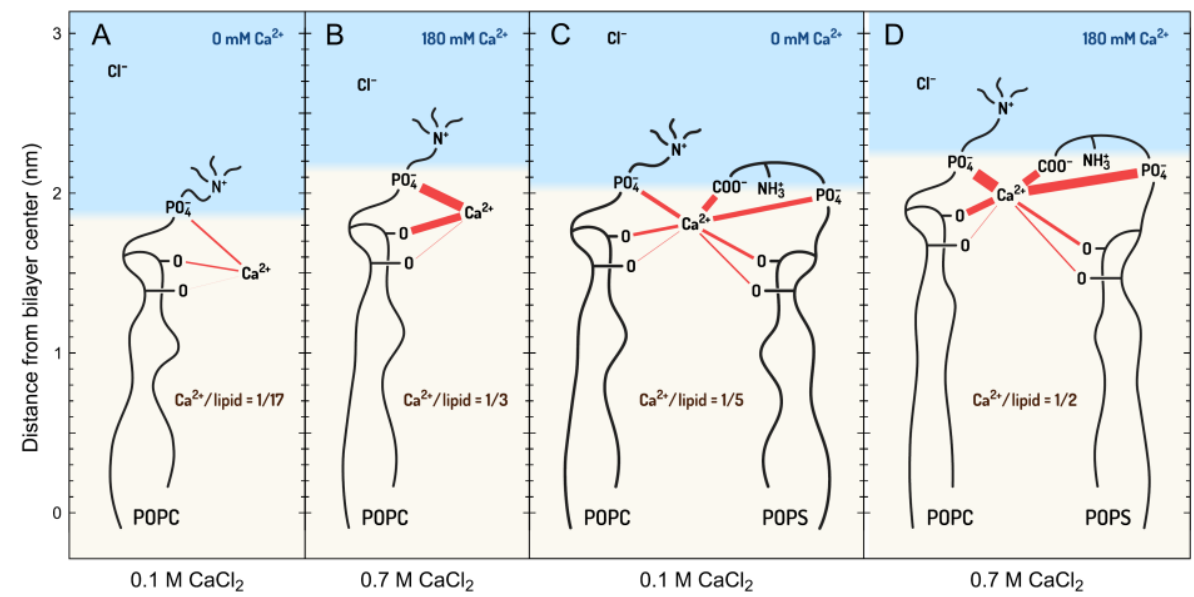

Figure 4.24 Schematic representation of the $\mathrm{Ca}^{2+}$-binding to the lipid headgroups $\mathrm{PC}$ and PC/PS depending on $\mathrm{Ca}^{2+}$ concentration. The graphic represents calculated values. Republished under the Creative Commons (CC BY 4.0) license. ${ }^{[188]}$

Alongside the theoretical examinations, the investigations of interactions between $\mathrm{Ca}^{2+}$ and the membrane surfaces are of interest. Apart from methods such as dynamic light scattering (DLS) or X-ray diffraction measurements, ${ }^{[258]}$ other methods are available to investigate near-membrane $\mathrm{Ca}^{2+}$. The biocompatible and well-established fluorescence spectroscopy provides the opportunity to use particular developed sensors, which detect $\mathrm{Ca}^{2+}$ and allow an attachment close to the membrane surface by membrane anchoring. ${ }^{[264]}$

\subsubsection{Fluorescence-based $\mathrm{Ca}^{2+}$-sensors}

A wide range of fluorescence sensors have been developed to measure the most relevant biological alkali metal ions $\left(\mathrm{Li}^{+}, \mathrm{Na}^{+}, \mathrm{K}^{+}\right)$and alkaline earth metal ions $\left(\mathrm{Mg}^{2+}, \mathrm{Ca}^{2+}\right) .{ }^{[123]}$ The basic structure of fluorescence-based ion-sensors consists of a ligand and a fluorophore. The ligand binds selectively to the respective ion, which affects the fluorescence properties of the fluorophore. ${ }^{[265]}$ Derivatives of ethylene glycol-bis(2aminoethylether)- $N, N, N^{\prime}, N^{\prime}$-tetraacetic acid (EGTA) were designed as selective $\mathrm{Ca}^{2+}$ ligands. Based on this, TSIEN developed 1,2-bis(o-aminophenoxy)ethane- $N, N, N^{\prime}, N^{\prime}$ tetraacetic acid (BAPTA) which shows a high affinity to $\mathrm{Ca}^{2+} \cdot{ }^{266]}$ Structurally, the ethylene bridge between the oxygen and the nitrogen of EGTA is replaced in BAPTA by benzene rings (see Figure 4.25). ${ }^{[266]}$ The development of BAPTA as a high-affinity ligand 
and optical indicator for $\mathrm{Ca}^{2+}$ analysis has been a major step in the detection of cellular $\mathrm{Ca}^{2+} \cdot[266]$

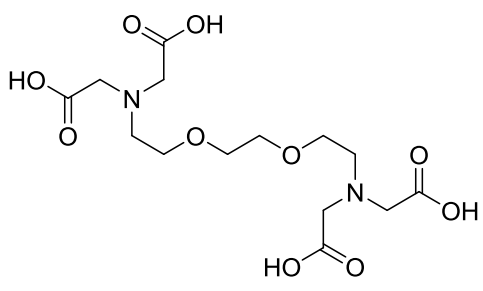

EGTA

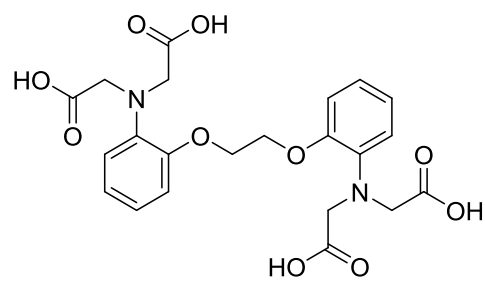

BAPTA

Figure 4.25 Structural formula of ethylene glycol-bis(2-aminoethylether)- $N, N, N^{\prime}, N^{\prime}$-tetraacetic acid (EGTA) and 1,2-bis(o-aminophenoxy)ethane- $N, N, N^{\prime}, N^{\prime}$-tetraacetic acid (BAPTA).

The structural modification of the ligand system leads to alterations of properties, like a shift in the basicity of the nitrogen atoms. ${ }^{[264]}$ The EGTA nitrogen atom has a $\mathrm{p} K_{\mathrm{a}}$ value of $8.5-9.5$. The aromatic rings of the BAPTA molecule change the $\mathrm{p} K_{\mathrm{a}}$ value to $6.0-$ 6.3. ${ }^{[264]}$ This basicity shift leads to a decisive change in the complexation of $\mathrm{Ca}^{2+}$ at a physiological $\mathrm{pH}^{[264]}$ Under these conditions, the nitrogen atoms of the BAPTA molecule are deprotonated, in contrast to the nitrogen atoms of the EGTA molecule which are protonated. Due to this, no displacement of a $\mathrm{H}^{+}$in the coordination sphere of BAPTA is required for the coordination of $\mathrm{Ca}^{2+}$, which accelerates the $\mathrm{Ca}^{2+}$ absorption of BAPTA in two or three orders of magnitude. ${ }^{[264]}$ Crystal structures of 5,5-difluorinated BAPTA with complexed $\mathrm{Ca}^{2+}$ show BAPTA as an octadentate ligand, where the four carboxyl groups, the oxygen atoms of the ethylene glycol bridge and the nitrogen atoms, which act as a LEWIS acid are involved. ${ }^{[267]}$ The functionalisation of the aromatic rings with substituents makes it possible to adjust the ligand binding properties by controlling the basicity of the coordinating nitrogen. ${ }^{[265]}$ Electron donating groups, like alkyl chains $\left(-\mathrm{C}_{n} \mathrm{H}_{2 n+1}\right)$ and heteroatoms $(\mathrm{N}, \mathrm{O})$, increase the basicity of the $\mathrm{Ca}^{2+}$-binding nitrogen, which leads to a higher affinity (smaller $K_{\mathrm{d}}$ ). Electron withdrawing groups, such as halogen atoms (-F, -Cl, $\mathrm{Br})$, nitro $\left(-\mathrm{NO}_{2}\right)$ and nitrile $(-\mathrm{CN})$ groups lead to a decrease in the basicity and to a lower binding affinity (larger $K_{\mathrm{d}}$ ). ${ }^{[265]}$ This allows the synthesis of a variety of BAPTA-based ligands with a broad distribution of $K_{\mathrm{d}}$ values. For additional ligand motifs with modified $K_{\mathrm{d}}$ values, one of the aromatic rings can be replaced by other functionalisation (see Figure 4.26). However, the thereof developed aminophenol triacetic acid (APTRA) does not show the desired affinity for $\mathrm{Ca}^{2+}$ and is also not selective towards other divalent ions such as $\mathrm{Mg}^{2+}{ }^{265]}$ In contrast, 2-(2'-morpholino-2'-oxoethoxy)- $N, N$-bis(hydroxycarbonylmethyl)aniline (MOBHA) is a possible chelating unit for low affinity $\mathrm{Ca}^{2+}$-sensors. ${ }^{[123,268,269]}$ 
<smiles>O=C(O)CN(CC(=O)O)c1ccccc1OCCOc1ccccc1N(CC(=O)O)CC(=O)O</smiles>

BAPTA<smiles>O=C(O)COc1ccccc1N(CC(=O)O)CC(=O)O</smiles><smiles>O=C(O)CN(CC(=O)O)c1ccccc1OCC(=O)N1CCOCC1</smiles>

\section{MOBHA}

Figure 4.26 Structural formula of 1,2-bis(o-aminophenoxy)ethane- $N, N, N^{\prime}, N^{\prime}$-tetraacetic acid (BAPTA), aminophenol triacetic acid (APTRA), 2-(2'-morpholino-2'-oxoethoxy)- $N, N$-bis(hydroxycarbonylmethyl)aniline (MOBHA).

BAPTA shows a fluorescence maximum in the range of $\sim 250 \mathrm{~nm}$ which is shifting by $\mathrm{Ca}^{2+}$-binding to two maxima in a range of $200-300 \mathrm{~nm}$ (see Figure 4.27). ${ }^{[270]}$ These fluorescence maxima result in an excitation wavelength $<200 \mathrm{~nm}$, which is highly toxic for biological systems. Due to the need of new biological compatible $\mathrm{Ca}^{2+}{ }_{\text {-sensors, it is }}$ necessary to shift the fluorescence wavelength to higher wavelengths.

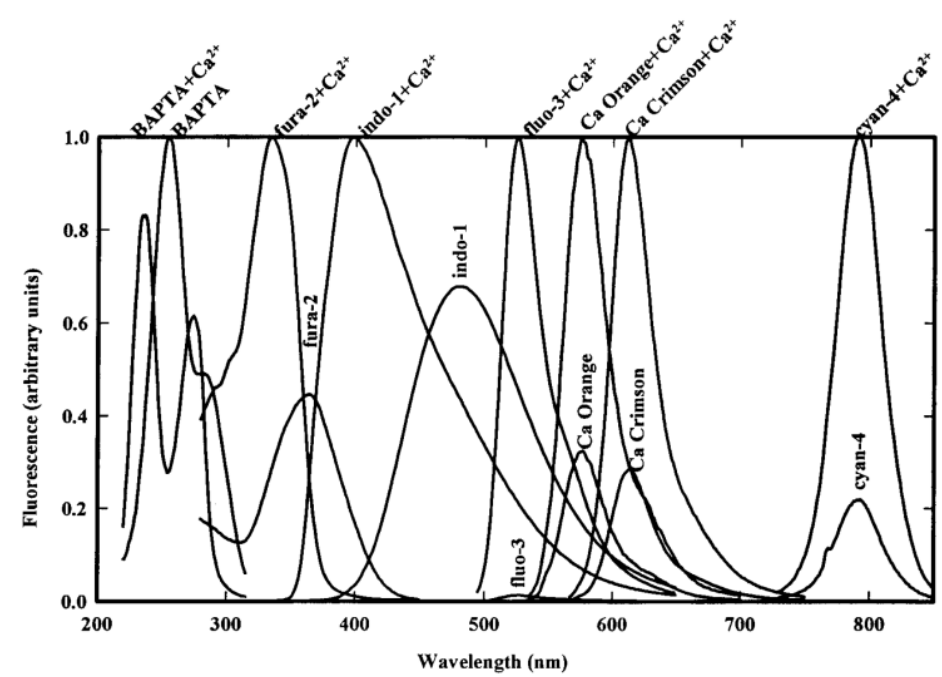

Figure 4.27 Fluorescence spectra of different $\mathrm{Ca}^{2+}$-sensors with and without $\mathrm{Ca}^{2+} \cdot{ }^{[270]}$ Reprinted from E. Carafoli, C. B. Klee, Calcium as a Cellular Regulator, Copyright (1999) Oxford University Press. Reproduced with permission of the Licensor through PLSclear

Such a fluorescence shift can be obtained by the attachment of a fluorophore to the chelating unit. ${ }^{[264]}$ This attachment leads to a change in the electronic system of BAPTA and the $\mathrm{Ca}^{2+}$-binding effects transfer to the electronic system of the fluorophore. ${ }^{[264,270]} \mathrm{In}$ Figure 4.28, variations of $\mathrm{Ca}^{2+}$-sensors based on BAPTA are shown. ${ }^{[265]}$ The used fluorophores of the presented $\mathrm{Ca}^{2+}$-sensors are attached via different motifs. 
<smiles></smiles>

Fura-red<smiles></smiles>

Calcium orange<smiles>CN(C)c1ccc2c(c1)Oc1cc([N+](C)(C)C)ccc1-c1ccc(N(CC(=O)O)CC(=O)O)c(OCCOc3ccccc3N(CC(=O)O)CC(=O)O)c1-2</smiles>

Rhod-2

\section{STDBT}

Figure 4.28 Selection of $\mathrm{Ca}^{2+}$-sensors. ${ }^{[265]}$

Two different working principles of the fluorescent $\mathrm{Ca}^{2+}$-sensors were found, the photoinduced electron transfer (PET) and the photoinduced charge transfer (PCT). ${ }^{[113,265,271]}$ PET based sensors have an electron donating group (D) in their structure which is part of the ligand system. In the BAPTA ligand, D is represented by the nitrogen atoms. The $\mathrm{D}$ is connected by a bridge to the conjugated aromatic system of a fluorophore and due to an electron transfer, the fluorescence of the fluorophore is quenched by the D (see Figure 4.29). ${ }^{[13,265,271]}$ An example for this type of $\mathrm{Ca}^{2+}$-sensors is Rhod-2, shown in Figure 4.28. In the $\mathrm{Ca}^{2+}$ free case, an electron from the highest occupied molecular orbital (HOMO) of the fluorophore is excited to the lowest unoccupied molecular orbital (LUMO) of the fluorophore. Subsequently, the D transfers an electron from its HOMO to the partially filled HOMO of the fluorophore in a radiation free manner (see Figure 4.29). ${ }^{[113,265,271]}$ 


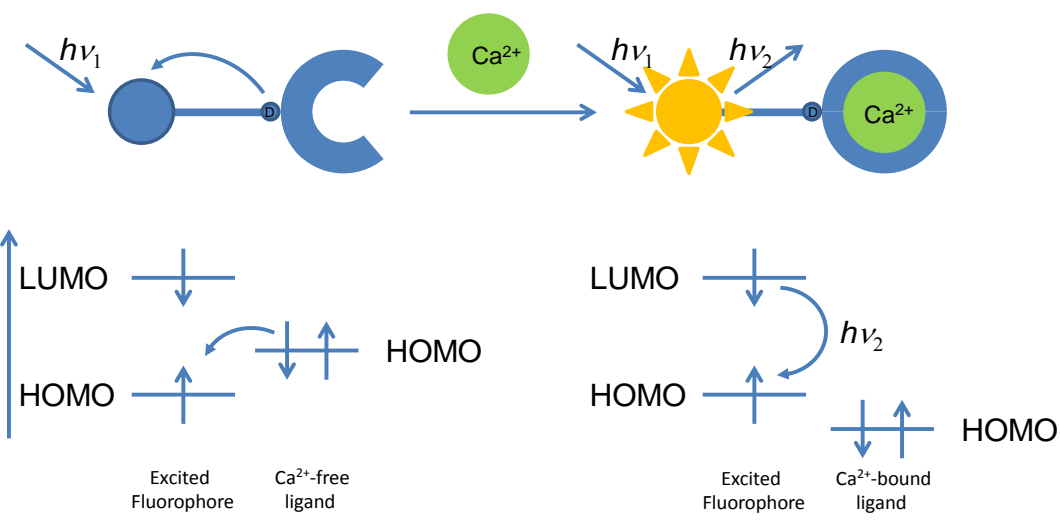

Figure 4.29 Schematic functional principle of a PET based $\mathrm{Ca}^{2+}$-sensors. After fluorophore excitation in the $\mathrm{Ca}^{2+}$ unbound state, the fluorescence is suppressed by electron transfer of the $\mathrm{D}$ to the fluorophore. The binding of $\mathrm{Ca}^{2+}$ leads to an increase of the fluorescence, due to the missing electron transfer of the $D .^{[113,265,271]}$ Graphic in accordance to YAN and OHEIM et al. ${ }^{[265,271]}$

The binding of $\mathrm{Ca}^{2+}$ decreases the energy of the HOMO from the nitrogen atoms (D), which serves as a LEWIS base in the $\mathrm{Ca}^{2+}$-binding and an electron transfer process is no longer possible. ${ }^{[113,265,271]}$ This inhibited electron transfer from the D to the fluorophore results in an increased fluorescence, which allows the determination of the $\mathrm{Ca}^{2+}$ concentration. The $\mathrm{Ca}^{2+}$ concentration can be calculated according to the following Formula 4.4 by knowing the $K_{\mathrm{d}}$, the minimal fluorescence $\left(\mathrm{F}_{\min }\right)$ and the maximum fluorescence $\left(\mathrm{F}_{\max }\right){ }^{[265,271]}$

$$
\left[\mathrm{Ca}^{2+}\right]=K_{\mathrm{d}}\left(\mathrm{Ca}^{2+}\right) \cdot\left(\mathrm{F}-\mathrm{F}_{\min }\right) /\left(\mathrm{F}_{\max }-\mathrm{F}\right)
$$

The $K_{\mathrm{d}}$ value varies with the ion strength, $\mathrm{pH}$ and temperature of the used solution. Therefore, a natural fluctuation of the $K_{\mathrm{d}}$ is present in complex biological systems. ${ }^{\text {[265,271] }}$ The PET type sensors are also named non-ratiometric sensors because the evaluation considers only one excitation wavelength and one emission wavelength. ${ }^{[265,271]}$

The PCT based $\mathrm{Ca}^{2+}$-sensors show, in contrast to the PET based $\mathrm{Ca}^{2+}$-sensors, a different fluorophore attachment to the ligand unit. The fluorophore is directly linked to the $\mathrm{Ca}^{2+}$ coordinating unit without a bridge. ${ }^{[13,265,271]}$ Here, the fluorophore can be an electron acceptor (A) or an electron donating group (D) and is connected to the electronic counterpart, the $\mathrm{Ca}^{2+}$-binding unit, via an aromatic system (see Figure 4.30). ${ }^{[113,265,271]}$ Depending on the distribution of $\mathrm{D}$ and $\mathrm{A}$ in the sensor, different fluorescence behaviours are observed by binding $\mathrm{Ca}^{2+}$. As a result of the structure, an outright charge transfer process from D to A takes place at an excitation of the aromatic system in an unbound 
state. ${ }^{[113,265,271]}$ The binding of $\mathrm{Ca}^{2+}$ alters the energy levels and affects the properties of the fluorophore. It is distinguished between the role of the chelator and the fluorophore as D or A. If the chelator acts as an $\mathrm{A}$, the binding of $\mathrm{Ca}^{2+}$ leads to a decrease of the energy levels $S_{0}$ and $S_{1}$ (see Figure 4.30 a). Here, a greater decrease of the $S_{1}$ state occurs, which results in a red shift in the absorption and the fluorescence spectra. The opposite case shows a blue shift of the spectra (see Figure $4.30 \mathrm{~b}$ ). In addition, the PST principle may result in a change in molar extinction $(\varepsilon)$, fluorescence quantum yields $\left(\Phi_{\mathrm{F}}\right)$ and excited-state lifetime $(\tau) .^{[113,265,271]}$

a)
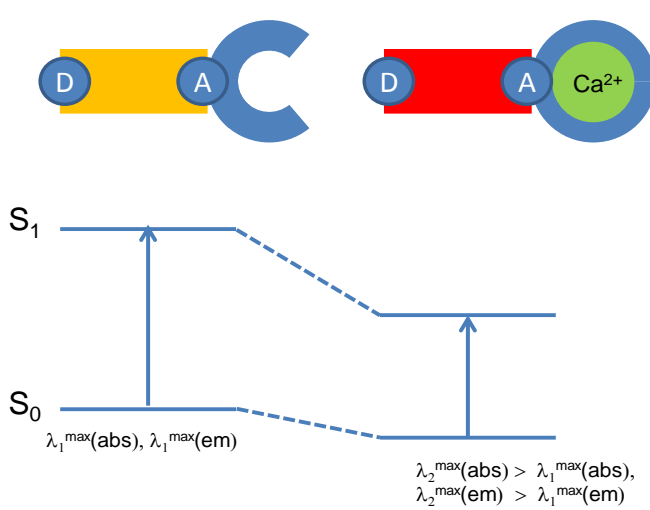

b)
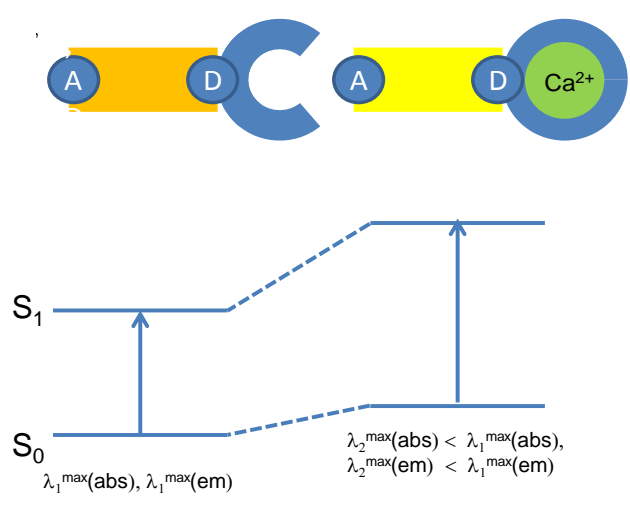

Figure 4.30 Schematic functional principle of a PCT based $\mathrm{Ca}^{2+}$-sensors. The binding of $\mathrm{Ca}^{2+}$ induced a energy change of the ground state $\left(S_{0}\right)$ and the excited state $\left(S_{1}\right)$. Depending on the $A / D$ distribution a) a decrease of the energy difference between $S_{0}$ and $S_{1}$ results from the $\mathrm{Ca}^{2+}$ binding and leads to a red shift of the fluorescence or $b$ ) an increase of the energy difference between $S_{0}$ and $S_{1}$ results from the binding of $\mathrm{Ca}^{2+}$ and leads to a blue shift of the fluorescence. ${ }^{[113,265,271]}$ Graphic is in accordance to YAN and OHEIM $e t$ al. ${ }^{[265,271]}$

To determine the $\mathrm{Ca}^{2+}$ concentration, two different absorption or emission wavelengths are observed and a ratiometric value $\left(\mathrm{R}=\mathrm{F}\left(\lambda_{1}\right) / \mathrm{F}\left(\lambda_{2}\right)\right)$ is calculated. From the respective ratios of $\mathrm{Ca}^{2+}$-free $\left(\mathrm{R}_{\min }\right)$ and $\mathrm{Ca}^{2+}$-saturated $\left(\mathrm{R}_{\max }\right)$, the following Formula 4.5 shows the relationship of the $\mathrm{Ca}^{2+}$ concentration. ${ }^{[265,271]}$

$$
\left[\mathrm{Ca}^{2+}\right]=\left(\mathrm{R}-\mathrm{R}_{\min }\right) /\left(\mathrm{R}_{\max }-\mathrm{R}\right) \cdot \mathrm{F}_{\min }\left(\lambda_{2}\right) / \mathrm{F}_{\max }\left(\lambda_{2}\right)
$$

These types of $\mathrm{Ca}^{2+}$-sensors are called ratiometric sensors because a ratio of two different absorption or emission wavelengths is calculated. ${ }^{[265,271]}$ Compared to the non-ratiometric sensors, the ratiometric sensors show less sensitivity to factors that can influence the fluorescence measurements. Such factors include the dye concentration, the optical path length and the sensitivity of the instrument. ${ }^{[265]}$ A dependency of the sensors of the solvent polarity, ionic strength, viscosity and temperature is still existing. However, in the literature, the non-ratiometric sensors like Fura-2 are used for most experiments. ${ }^{[265]}$ Based 
on optimised fluorescence measurements, the ratiometric sensors could be more common in the future due to their advantages.

\subsubsection{Design strategies of new near-membrane $\mathrm{Ca}^{2+}$-sensors}

The labelling of biomolecules is a part of many studies and some of these labelling techniques are so ubiquitousthat specific kits are commercially available. ${ }^{[113,223]}$ The attachment of fluorophores can be performed by various coupling methods which differ in the used functionalities, the bioorthogonality and the synthetic effort. A biomolecule is often labelled at a functional group with a chemically activated fluorophore. ${ }^{[117,118,272]}$ This activation has to be orthogonal to other functional groups and leads to a covalent bond. A variety of methods has been developed ranging from nonspecific methods that are labelling functional groups to very specialised labelling reactions, which are mediated through a particular sequence or an artificial unnatural amino acid. ${ }^{[117,118,272]}$

Many processes in biological systems depend on varying $\mathrm{Ca}^{2+}$ concentrations that interact with the membrane surfaces (see Chapter 4.2.2). Although most of the $\mathrm{Ca}^{2+}$-sensors are designed as water soluble sensors, a minority of the known sensors have been developed as near-membrane $\mathrm{Ca}^{2+}$-sensors. ${ }^{[264]}$ Here, the anchoring in the membrane often takes place via a fatty acid chain (see Figure 4.31). ${ }^{[35-37,264]}$ Due to the strong interaction of $\mathrm{Ca}^{2+}$ with the membrane, higher $\mathrm{Ca}^{2+}$ concentrations are observed near the membrane compared to the cytosol. ${ }^{[35,188,264]}$ To determine these elevated $\mathrm{Ca}^{2+}$ concentrations, the saturation limit of the sensors has to be increased compared with water soluble $\mathrm{Ca}^{2+}$-sensors. Therefore, new near-membrane $\mathrm{Ca}^{2+}$-sensors with a higher $K_{\mathrm{d}}$ are synthesised. ${ }^{[35-37,264]}$ In addition to the increased $\mathrm{Ca}^{2+}$ concentration, the membrane itself can lead to interaction with the fluorophore of the sensor, wherefore a low flexibility between the sensor and the membrane anchor can be advantageous. ${ }^{[211]}$ The $\mathrm{Ca}^{2+}$-sensors shown in Figure 4.31 are characterised by a relatively high $K_{\mathrm{d}}$ as well as a fatty acid chain modification which takes place as far as known in the last reaction of the synthesis. ${ }^{[37,273]}$ The anchoring via a fatty acid chain is widespread, though it does not allow a rigid elongation in selectable distances to the membrane. Nevertheless, these sensors could serve as the basis for new easily modifiable $\mathrm{Ca}^{2+}$-sensors. 
<smiles>CCCCCCCCN1CCN(C(=O)CCc2ccc(N(CC(=O)O)CC(=O)O)c(OCCOc3cc4oc(-c5ncc(C(=O)O)o5)cc4cc3N(CC(=O)O)CC(=O)O)c2)CC1</smiles>

Fura-2 NearMem (FFP-18) $\mathrm{R}_{\mathrm{Ex}}=\mathrm{F}_{340} / \mathrm{F}_{380}$ $K_{\mathrm{d}}=0.4 \mu \mathrm{M}$

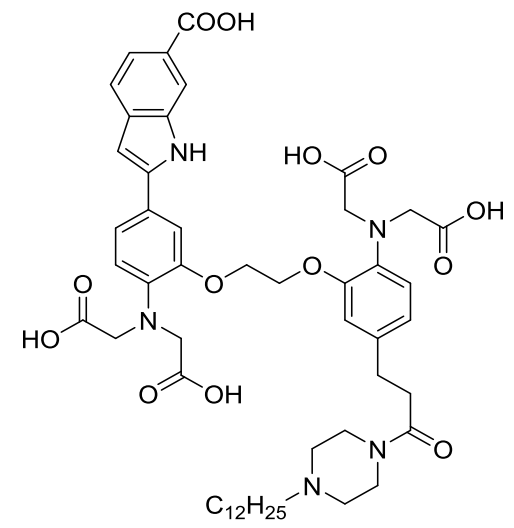

Indo-1 NearMem (FIP-18) ${ }^{[36]}$ $\lambda_{\mathrm{Ex}}=514 \mathrm{~nm}$ $\lambda_{\mathrm{Em}}=5540 \mathrm{~nm}$ $K_{\mathrm{d}}=0.1 \mu \mathrm{M}$

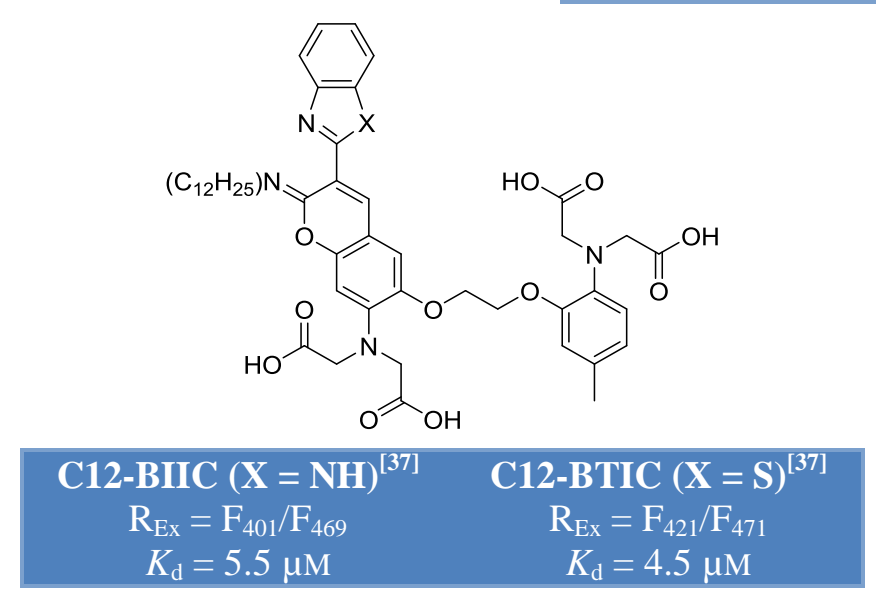

Figure 4.31 Chemical structure and physical properties of membrane-bound $\mathrm{Ca}^{2+}$-sensors.

GRAF was able to synthesise several $\mathrm{Ca}^{2+}$-sensors to expand the application areas of $\mathrm{Ca}^{2+}$-sensors. ${ }^{[274]}$ These $\mathrm{Ca}^{2+}$-sensors were modified with an azide group (see Figure 4.32). This modification made it possible to bind the $\mathrm{Ca}^{2+}$-sensor to a polyethylene glycol (PEG) motif by a HUISGEN cycloaddition. ${ }^{[275,276]}$ HANSEN labelled lipid and cholesterol derivatives with the $\mathrm{Ca}^{2+}$-sensor developed by GRAF, but the attachment to short peptide sequences was not successful. ${ }^{[277]}$ The introduction of $\mathrm{Ca}^{2+}$-sensors with reactive groups as connection point shown by GRAF, represents a promising approach. Since the azide functionality is incorporated in the middle of the synthesis, the implementation of modifications in the system is difficult based on this synthesis strategy. ${ }^{[274]}$

The goal of this work is the development of a $\mathrm{Ca}^{2+}$-sensor, which can be easily modified, primarily for labelling transmembrane $\beta$-peptides. Therefore, 3-(benzimidazolyl)iminocoumarin (BIIC) and 3-(benzothiazolyl)iminocoumarin (BTIC) moieties were used as basic structures (see Figure 4.31). ${ }^{[37,278]}$ Here, the incorporation of the fluorophore and 
the functionalisation with a $\mathrm{C}_{12}$ alkyl chain occurred in the last steps of the synthesis. These sensor properties and the $\beta$-peptide specific properties like the length of the peptide, the polarity and the structure enable a new range of $\mathrm{Ca}^{2+}$ detecting tools.

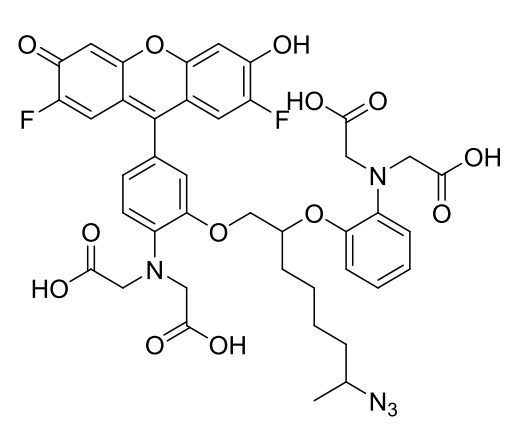

$$
\begin{gathered}
\text { Fluo-Azid-1 } \text { [274] } \\
\begin{aligned}
\lambda_{\mathrm{Ex}} & =493 \mathrm{~nm} \\
\lambda_{\mathrm{Em}} & =514 \mathrm{~nm} \\
K_{\mathrm{d}} & =1.5 \mu \mathrm{M}
\end{aligned}
\end{gathered}
$$<smiles></smiles>

Fluo-Arid-2 $2^{[274]}$

$\lambda_{\mathrm{Ex}}=493 \mathrm{~nm}$

$\lambda_{\mathrm{Em}}=514 \mathrm{~nm}$

$K_{\mathrm{d}}=0.7 \mu \mathrm{M}$

Figure 4.32 Chemical structure and physical properties of azide functionalised $\mathrm{Ca}^{2+}$-sensors developed by GRAF. ${ }^{[274]}$

\subsubsection{Synthesis and measurements}

Inspired by the synthesis of $\mathrm{C}_{12}$-BIIC (see Figure 4.31 and Scheme 4.1), different kinds of BIIC modifications were tested. The tentative idea was to address different biochemical functional groups in peptides, like the $-\mathrm{NH}_{2}$ group of lysine or the $-\mathrm{SH}$ group of cysteine side chains. ${ }^{[116-118]}$ After the successful modification of the literature known methyl protected BIIC, two tert-butyl protected $\mathrm{Ca}^{2+}$-sensors based on BTIC were synthesised (6 and 7). The change of the protection strategy permitted the use in Fmoc-based SPPS. The replacement of benzimidazol with benzothiazolyl prevents a side reaction of the activated $\mathrm{Ca}^{2+}$-sensor in the SPPS with the -NH group of the benzimidazol. Both synthesised tert-butyl protected $\mathrm{Ca}^{2+}$-sensors differ in the used chelator unit, which were either BAPTA $(\mathbf{8}, \mathbf{6})$ or MOBHA $(7$, see Figure 4.33$) .{ }^{[123]}$

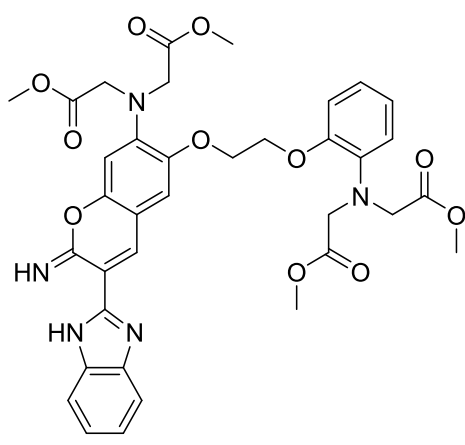

8

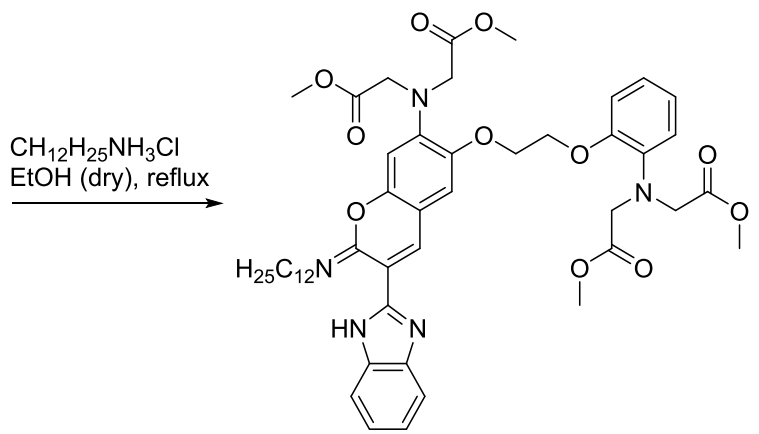

9

Scheme 4.1 Functionalisation of the methyl protected BIIC 8 with a $C_{12}$ alkyl chain lead to the protected near-membrane $\mathrm{Ca}^{2+}$-sensor 9. ${ }^{[37]}$ 
The BAPTA-based $\mathrm{Ca}^{2+}$-sensor was synthesised according to the literature as methyl protected $\mathbf{8}$ and as tert-butyl protected $\mathbf{6}$ and the tert-butyl protected MOBHA $\mathrm{Ca}^{2+}$-sensor 7 was synthesised inspired by the synthesis of Calcium Green FIAsH developed by TouR et al. (Figure 4.42). ${ }^{[37,269,278,279]}$
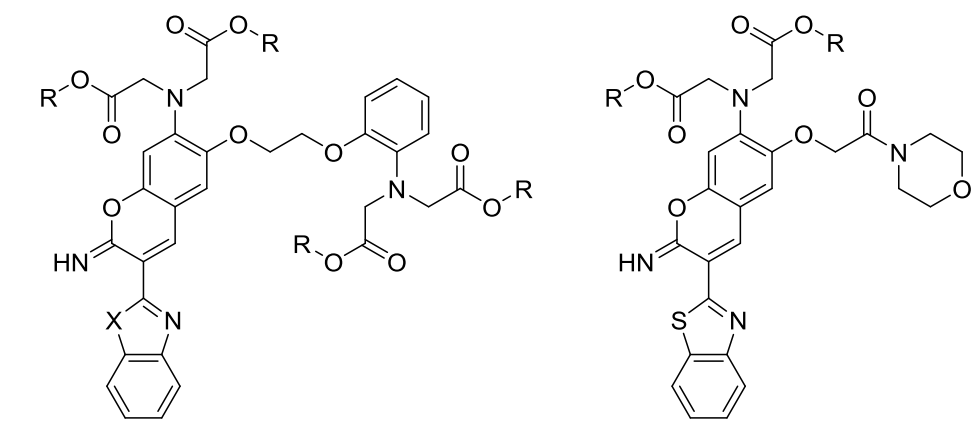

$$
\begin{aligned}
& X=N, R=M e(8) \\
& X=S ; R=t \operatorname{Bu}(\mathbf{6}) \\
& X=S ; R=H(10)
\end{aligned}
$$

$$
\begin{aligned}
& \mathrm{R}=t \mathrm{Bu}(\mathbf{7}) \\
& \mathrm{R}=\mathrm{H}(\mathbf{1 1})
\end{aligned}
$$

Figure 4.33 Basic structure of the BAPTA-based $\mathrm{Ca}^{2+}$-sensor 8 and 6 as well as the MOBHA-based $\mathrm{Ca}^{2+}$-sensor 7 .

The starting nitrophenol 12 forms the basis of all following $\mathrm{Ca}^{2+}$-sensors. Therefore, in the first step, hydroquinone $\mathbf{1 3}$ was protected with benzyl bromide $\mathbf{1 4}$ in the presence of potassium carbonate to 1,4-bis(benzyloxy)benzene 15. The diprotected nitrobenzene $\mathbf{1 6}$ was obtained by the reaction of $\mathbf{1 5}$ with acetic acid and nitric acid. 16 was selective deprotected at ortho position by using aluminium chloride to give 12 (see Scheme 4.2). ${ }^{\text {[280] }}$

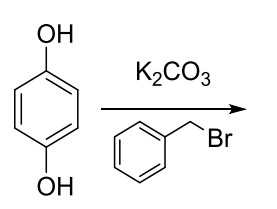

1314

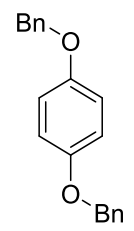

15

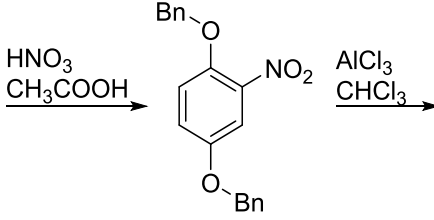

16<smiles>O=[N+]([O-])c1cc(O)ccc1O</smiles>

12

Scheme 4.2 Synthetic rout for the preparation of 4-benzyloxy-2-nitrophenol 12.

The monoprotected compound $\mathbf{1 2}$ was treated with the commercially available 1-(2bromoethoxy)-3-nitrobenzene 17 to obtain the bisnitro compound $\mathbf{1 8}$. Thus, the phenolic alcohol of 12 was deprotonated with $\mathrm{K}_{2} \mathrm{CO}_{3}$ and in a nucleophilic substitution reaction the bisnitro compound 18 was yielded. By a BÉCHAMP reduction, the bisnitro compound 18 was converted into the diamine compound 19 (see Scheme 4.3). ${ }^{[279,281]}$ This diamine compound was the starting substance for the subsequently synthesised basic labile (8) and acid-labile (6) BAPTA units. 
<smiles>CC(C)(C)[N+](=O)[O-]</smiles>

12

17<smiles>O=[N+]([O-])c1ccccc1OCCOc1ccc(OCc2ccccc2)cc1[N+](=O)[O-]</smiles>

18

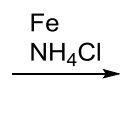<smiles>Nc1ccccc1OCCOc1ccccc1N</smiles>

19

Scheme 4.3 Synthetic rout for the preparation of 2-[2'-(2',-Aminophenoxy)ethoxy]-5-(benzyloxy)aniline 19 as the basic structure for the methyl and tert-butyl protected BAPTA motifs.

\section{Synthesis and modification of alkaline labile $\mathrm{Ca}^{2+}$-sensors based on BAPTA}

The methyl protected BAPTA $\mathrm{Ca}^{2+}$ sensor was synthesised analogously to the literature. Furthermore, the modifiability was tested with four different functional groups. ${ }^{[37]}$ Therefore, the diamine compound $\mathbf{1 9}$ was converted with methyl bromacetate $\mathbf{2 0}$ to the methyl protected BAPTA unit 21. The selective introduction of an aromatic aldehyde group to $\mathbf{2 1}$ was performed by a VILSMEIER-HAACK reaction and leads to $\mathbf{2 2}$ (Scheme $4.4){ }^{[282]}$<smiles>Nc1ccccc1OCCOc1ccc(OCc2ccccc2)cc1N</smiles>

19<smiles>COC(=O)CN(CC(=O)OC)c1ccccc1OCCOc1cc(C=O)c(O)cc1N(CC(=O)OC)CC(=O)OC</smiles>

23

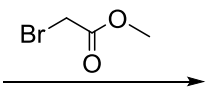

20

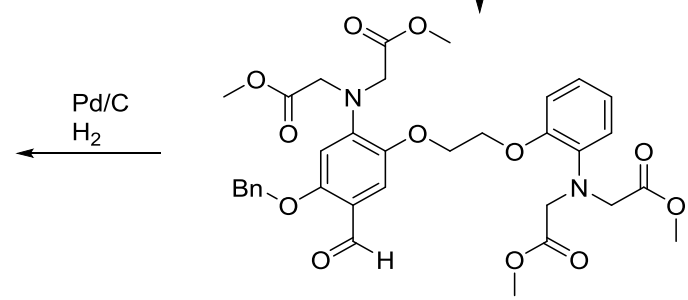

22

Scheme 4.4 Synthetic rout for the $o$-hydroxybenzaldehyde 23, which will be the precursor for the attachment of an fluorophore by KNOEVENAGEL condensation. ${ }^{[23,284]}$

Subsequently the aromatic benzyl protecting group was cleaved by $\mathrm{Pd} / \mathrm{C}$. In agreement with the original literature, the deprotection was carried out in acetic acid with $\mathrm{Pd} / \mathrm{C}$ (10\% wt). Firstly, a reaction with 0.5 bar $\mathrm{H}_{2}$ overpressure was tested overnight. This reaction condition resulted in a deprotection of the aromatic alcohol and also to a reduction of the aldehyde group (sees Scheme 4.5). ${ }^{[285]}$ Secondly, a reaction without $\mathrm{H}_{2}$ overpressure and a reaction time of less than $3 \mathrm{~h}$ was tested. TLC monitoring showed complete 
conversion of the educt after $3 \mathrm{~h}$. Furthermore, the mass spectrometric analysis indicated a partial conversion to the reduced product. Therefore, this reaction was carried out in comparison to the original literature with shortened times. ${ }^{[279]}$

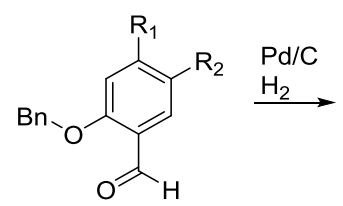<smiles>[R]c1cc(C)c(O)cc1[R2]</smiles>

Scheme 4.5 Pd(0)-catalysed hydrogenation of aromatic aldehyde group. ${ }^{[285]}$

The deprotected $o$-hydroxybenzaldehyde $\mathbf{2 3}$ and the cyanomethyl compound $\mathbf{2 4}$ reacted by a KNOEVENAGEL condensation to 8 (see Scheme 4.6). ${ }^{[283,284]}$ This reaction works in a wide range of cyanomethyl functionalised molecules. ${ }^{[286-288]}$

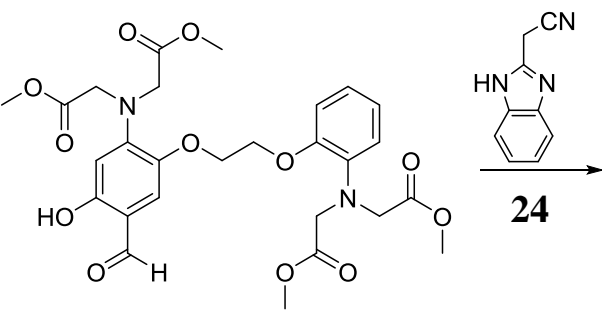

23

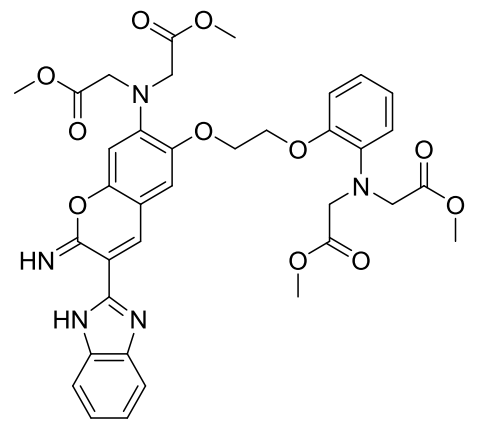

8

Scheme 4.6 Synthetic rout for the methyl protected $\mathrm{Ca}^{2+}$-sensor 8 in a KNOEVENAGEL condensation. ${ }^{[283,284,286-288]}$

The protected product $\mathbf{8}$ was used to test the range of the modifiability based on nitrogen substitution (see Scheme 4.1 and Scheme 4.7). ${ }^{[37]}$ This literature known nitrogen substitution modification of $\mathbf{8}$ (BIIC) was performed with the hydrochloride salt of dodecylamine. ${ }^{[37]}$ Due to this, for further modifications the hydrochloride salts of the reactant were used as well. On the basis of the tentative idea to address the $-\mathrm{NH}_{2}$ group of lysine or the - $\mathrm{SH}$ group of cysteine side chains, various sensor modifications were tested (see Scheme 4.7). The attachment of disulfide bridge forming molecules, like (S)-2pyridylthio cysteamine hydrochloride 25 and 2,2'-dithiobis(ethylamine) dihydrochloride 26, permitted it to equipped the $\mathrm{Ca}^{2+}$-sensor $\mathbf{8}$-SH selective functionalities. ${ }^{[16]}$ The thiol selective maleimide group also provided a motif frequently used in the literature for binding fluorophores to peptide cysteines. ${ }^{[113,116-118]}$ 


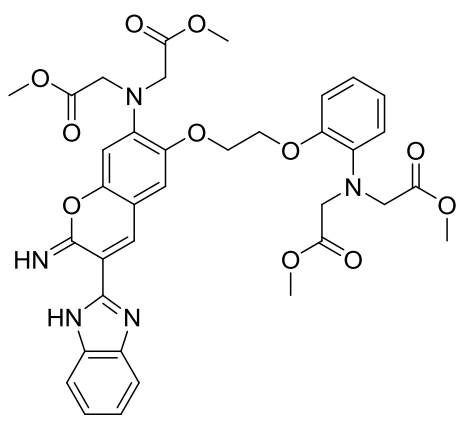

8

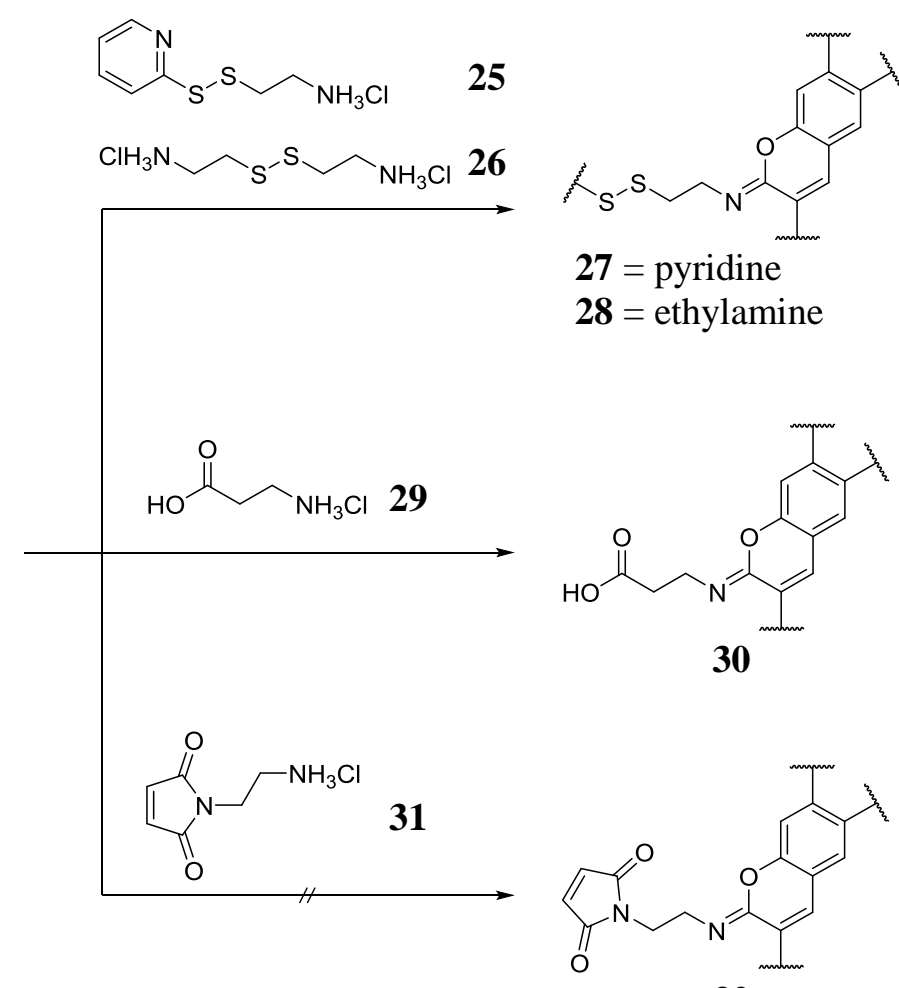

32

Scheme 4.7 Different modification of 8 by nitrogen substitution.

For application of $-\mathrm{NH}_{2}$ groups as target functionality, a modification with $\beta$-alanine hydrochloride 29 was tested. This modification allows among others, the usage of SPPS coupling methods. ${ }^{[289]}$ The successful conversion of the products $\mathbf{2 7}, \mathbf{2 8}$ and $\mathbf{3 2}$ were detected by mass spectrometry and the structures are shown in Scheme 4.7. The poor solubility in various solvents of $N$-(2-aminoethyl)maleimide hydrochloride $\mathbf{3 1}$ can lead to no reaction with $\mathbf{8}$. However, these results indicate the broad modification potential of the presented $\mathrm{Ca}^{2+}$-sensor basic structure. In order to use the sensor in the Fmoc based SPPS, the functionalisation with $\beta$-alanine was investigated in the following.

\section{Synthesis and modification of acid-labile $\mathrm{Ca}^{2+}$-sensors based on BAPTA and incorporation by SPPS to a water soluble $\beta$-peptide}

The functionalisation of $\mathbf{3 0}$ with $\beta$-alanine provides the carboxylic acid, which is required in the SPPS. However, the methyl protecting groups are not suitable for the Fmoc based SPPS, thus a synthesis route with tert-butyl groups was developed. ${ }^{[290]}$ The conversion of 19 with tert-butyl bromoacetate 33 enabled this synthesis and an overview of the synthesis is given in Scheme 4.8. The acid-labile protecting group tert-butyl was stable to the analogous synthesis conditions of the methyl protected derivate. As fluorophore, 2-benzothiazoleacetonitrile $\mathbf{3 4}$ was used instead of 2-benzimidazolylacetonitrile $\mathbf{2 4}$. This 
change was made to prevent interactions of the activated carboxylic acid during the SPPS with the NH group of the fluorophore.<smiles>CCOC(=O)CN(CC(=O)OCc1ccccc1)c1ccccc1OCCOc1ccc(OCC(=O)OCC(=O)OCc2ccccc2)c(N(CC(=O)OCc2ccccc2)CC(=O)OCc2ccccc2)c1</smiles>

19

33

35

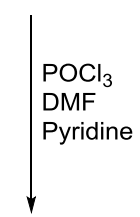

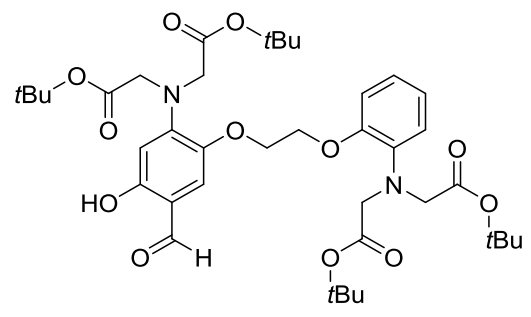

37

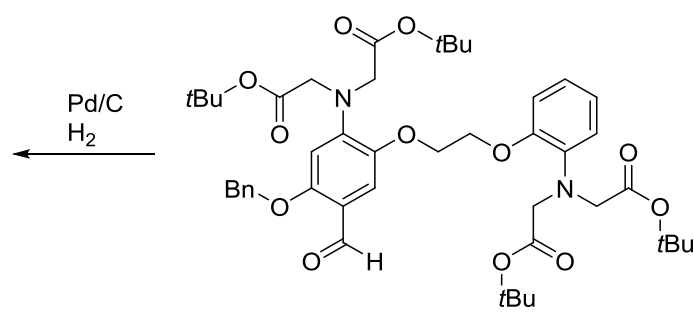

36

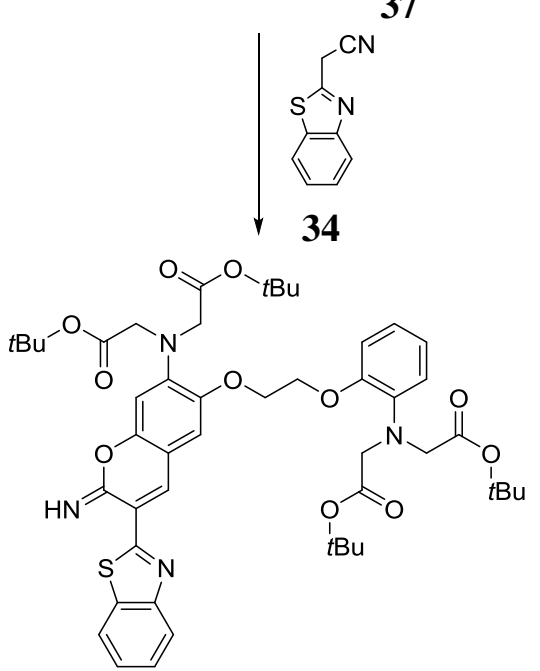

6

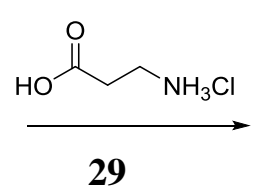

29

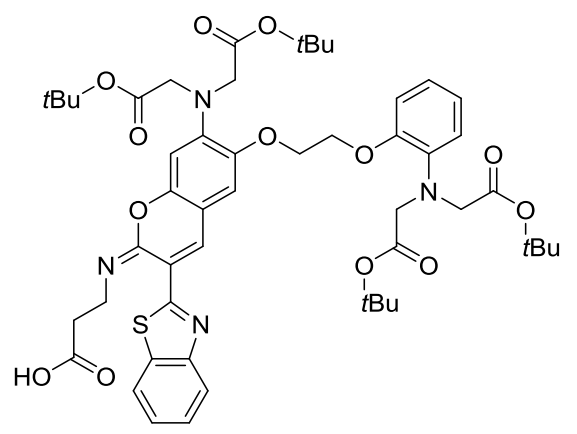

38

Scheme 4.8 Synthetic overview for the tert-butyl protected $\mathrm{Ca}^{2+}$-sensor 38 .

Iminocoumarin is known to show instability in acidic aqueous systems by hydrolysis (Scheme 4.9). ${ }^{[283,288]}$ Therefore, the stability of $\mathbf{3 8}$ was analysed in HPLC mixture of $\mathrm{H}_{2} \mathrm{O}$ and organic solvent $(\mathrm{MeOH}$ or $\mathrm{ACN})$ in a 50:50 ratio with 0.1\% TFA. In addition, a 50:50 ratio of $\mathrm{H}_{2} \mathrm{O}$ and $\mathrm{ACN}$ together with $1 \mathrm{M}$ TEAA buffer ( $\mathrm{pH}$ 7.0) was tested. Each mixture was shaken for $1 \mathrm{~h}$ at $\mathrm{RT}$ or $60{ }^{\circ} \mathrm{C}$, respectively. The solvent was subsequently lyophilised and the sample was examined by mass spectrometry. In the solvent mixture $\mathrm{H}_{2} \mathrm{O} / \mathrm{MeOH}+$ 
$0.1 \%$ TFA and $\mathrm{H}_{2} \mathrm{O} / \mathrm{ACN}+\mathrm{TEAA}$ at $60{ }^{\circ} \mathrm{C}$ mass spectrometric analysis have shown a conversion of the tert-butyl protected $\mathrm{Ca}^{2+}$-sensor $\mathbf{3 8}$ to the hydrolysed side product shown in Scheme 4.9.
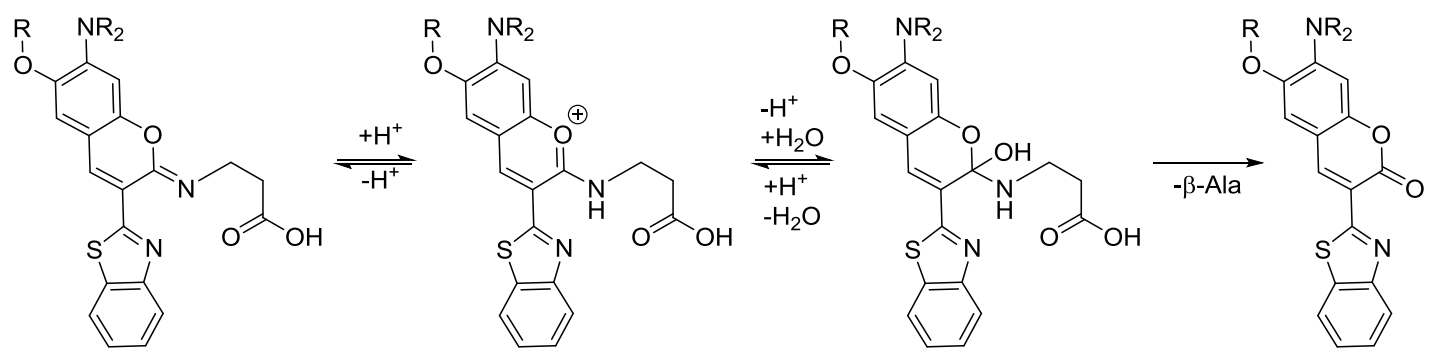

Scheme 4.9 Mechanism of the acid catalysed hydrolysation of the tert-butyl protected $\mathrm{Ca}^{2+}$-sensor $38 .{ }^{[283]}$

Regarding the stability in acidified aqueous solution at RT and previous results of fluorescein, different SPPS coupling conditions for $\mathbf{3 8}$ were tested. The fluorophore $\mathbf{3 8}$ (3.00 eq), PyAOP (6.00 eq) and HOAt (3.00 eq) were dissolved in DMF (15 mL/mmol based on resin-bound peptide) and DIPEA (6.00 eq) was added subsequently in three fractions to decrease heat development. This reaction mixture was added to the resin and if required DMF was added until the resin was completely covered with organic solvent. The fluorophore was coupled microwave-supported for $2 \mathrm{~h}$ to the resin-bound peptide $(20 \mathrm{~W}$, $2 \mathrm{~h}, 65^{\circ} \mathrm{C}$ (up to $10^{\circ} \mathrm{C}$ overshoot)). Because of the longer coupling time and the unknown efficiency of the reaction, PyAOP was used instead of HATU. The phosphonium salt PyAOP shows in contrast to the uronium salt HATU, no deactivation of a free $N$-terminus by guanidinylation. ${ }^{[291,292]}$ To perform the first characterisations of the peptide-bound $\mathrm{Ca}^{2+}$-sensor 38, an alternating sequence of $\mathrm{D}-\beta^{3}$-homovaline $\left(\mathrm{D}-\beta^{3}-\mathrm{hVal}\right)$ and $\mathrm{D}-\beta^{3}$-homolysine (D- $\beta^{3}$-hLys) was used. This sequence allowed the observation of the peptide-bound $\mathrm{Ca}^{2+}$-sensor Pep 18 in aqueous medium without peptide aggregation or lipid-peptide interactions.

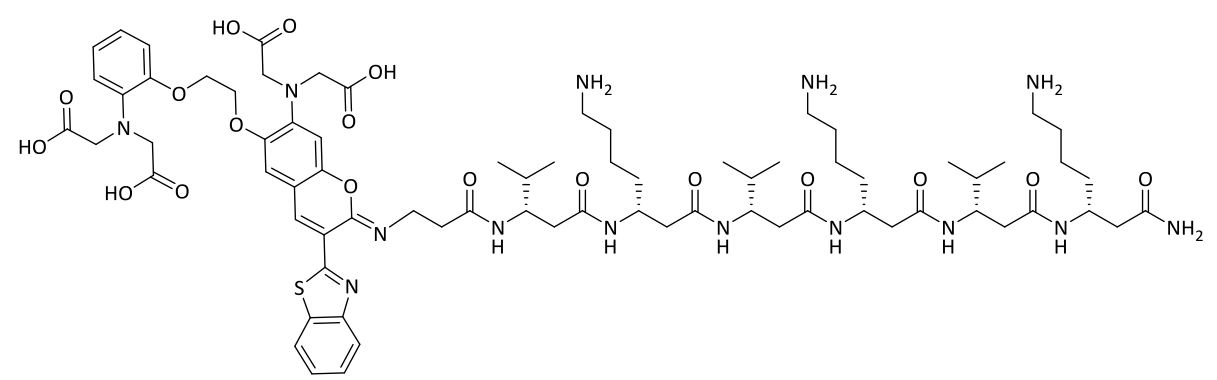

Figure 4.34 Sequence of peptide Pep 18.

Pep 18 
The peptide was cleaved from the resin with TFA/TIS (95:5) after the microwave-supported SPPS. A water free cleavage solution was used because water mediated the conversion of iminocoumarin to coumarin (see Scheme 4.9). The cleaved peptide was analysed by HPLC followed by mass spectrometric analysis. For this, the dried crude peptide was dissolved in water and examined with a gradient $30 \rightarrow 100 \% \mathrm{~B}(\mathrm{~A}$ : $\mathrm{H}_{2} \mathrm{O}+0.1 \%$ TFA and $\mathrm{B}: \mathrm{MeOH}+0.1 \%$ TFA). The HPLC chromatogram (see Figure 4.35) displays two intense peaks at $t_{\mathrm{R}}=15.9 \mathrm{~min}$ and $16.8 \mathrm{~min}$, which appear at the peptide bond specific wavelength $215 \mathrm{~nm}$ and at the fluorophore specific wavelength $480 \mathrm{~nm}$.

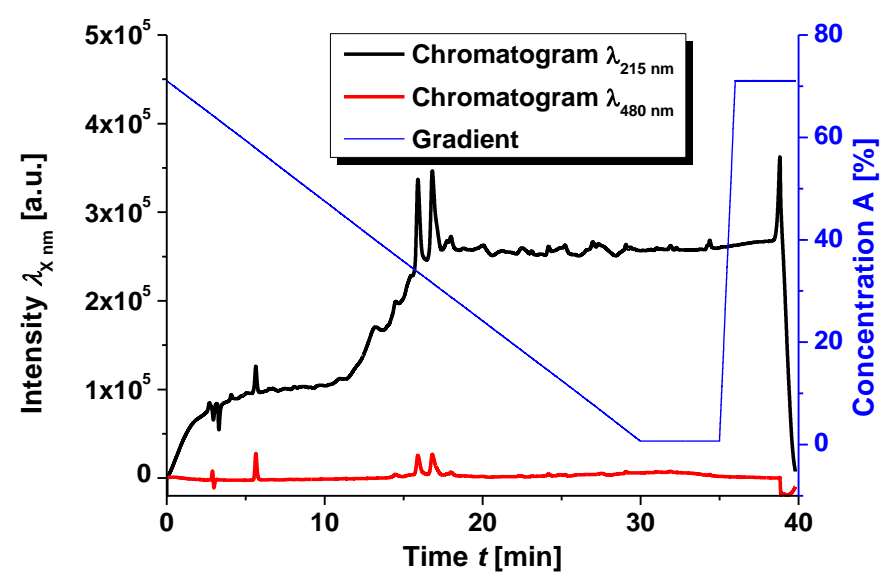

Figure 4.35 HPLC chromatogram of the crude peptide Pep 18. Absorption was recorded at $215 \mathrm{~nm}$ and $480 \mathrm{~nm}$. Analytical HPLC was performed using a gradient $30 \rightarrow 100 \%$ B $\left(\mathrm{A}: \mathrm{H}_{2} \mathrm{O}+0.1 \%\right.$ TFA and B: $\mathrm{MeOH}+$ $0.1 \%$ TFA) in $30 \mathrm{~min}$, flow $1.0 \mathrm{~mL} / \mathrm{min}$ and $25{ }^{\circ} \mathrm{C}$. The sample was dissolved in $100 \% \mathrm{H}_{2} \mathrm{O}$.

MS analysis of the two signals (see Figure 4.36) revealed that the peak at $t_{\mathrm{R}}=15.9 \mathrm{~min}$ is the desired product with a mass of $1512.8 \mathrm{~g} / \mathrm{mol}$ and the peak at $t_{\mathrm{R}}=16.8 \mathrm{~min}$ has a decreased mass of $1454.8 \mathrm{~g} / \mathrm{mol}[\mathrm{M}-2 \mathrm{C} 2 \mathrm{H} 2 \mathrm{O}]$.

a)

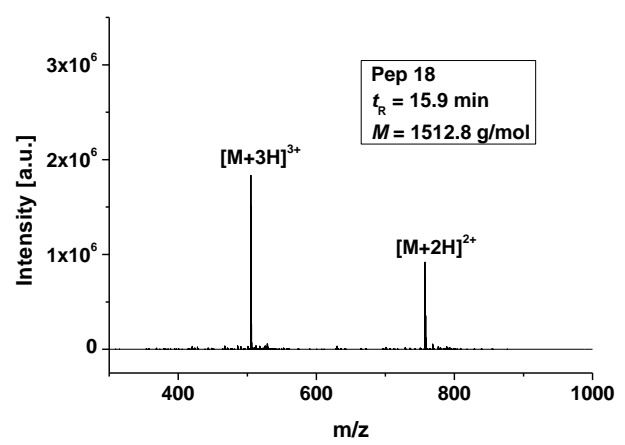

b)

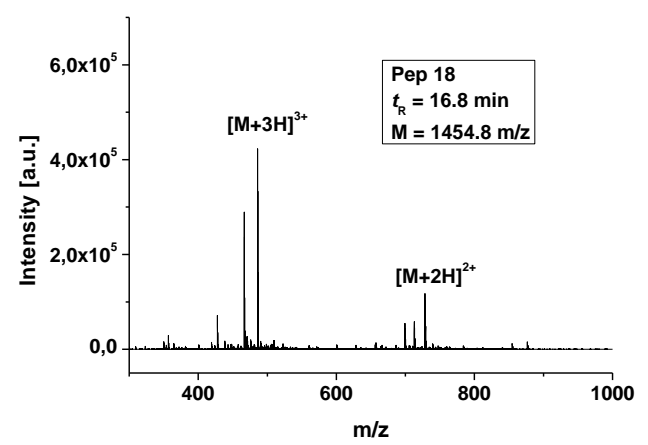

Figure 4.36 ESI mass spectra of Pep 18 at a) $t_{\mathrm{R}}=15.9 \mathrm{~min}$ and $\left.\mathrm{b}\right) t_{\mathrm{R}}=16.8 \mathrm{~min}$.

This mass difference can be explained by a defunctionalisation of the chelating carboxylic acids due to the cleavage process of the peptide from the resin. Pep 19 does not show this 
defunctionalisation, why it is assumed that at the non-fluorescent aniline of the sensor (see Scheme 4.10 highlighted in blue) the side reaction occurred. This effect could not be observed at the peptide-bound MOBHA-based $\mathrm{Ca}^{2+}$-sensor Pep 19.

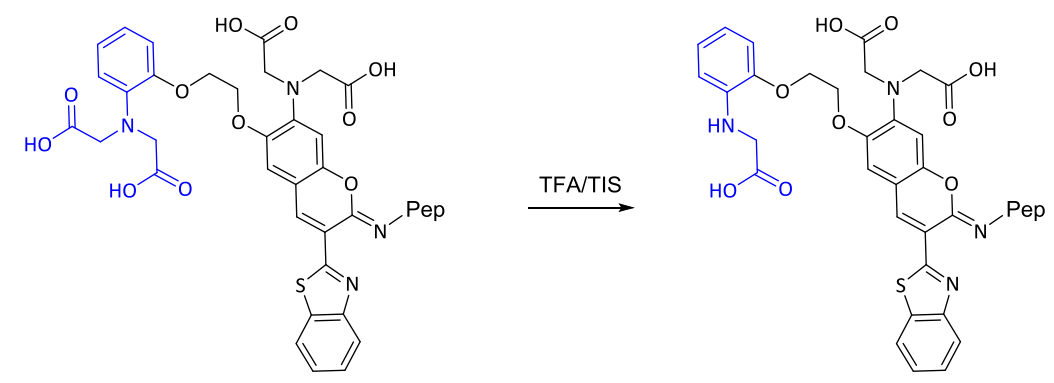

Scheme 4.10 Assumed defunctionalisation of Pep 18 during the cleavage of the resin.

The HPLC data revealed that besides the target product and a defunctionalised peptide, no further side effects can be observed. The conversion of iminocoumarin to coumarin, shown below in Scheme 4.9, could not be observed.

Following the successful synthesis of Pep 18 by SPPS, the different spectroscopic properties were investigated. As described in Chapter 4.2.3, the sensor presented here is a ratiometric sensor. The UV/Vis absorption spectra show a change in the absorption band with increasing $\mathrm{Ca}^{2+}$ concentration. In Figure 4.37, the absorption curve of Pep 18 is depicted for $0 \mu \mathrm{M} \mathrm{Ca}^{2+}$ and for $1 \mathrm{mM} \mathrm{Ca}^{2+}$. Therefore, an approximate consistent amount of Pep 18 in Zero Free $\mathrm{Ca}^{2+}$ buffer (10 mM K 2 EGTA, $100 \mathrm{mM} \mathrm{KCl}, 30 \mathrm{mM}$ MOPS, pH 7.2) from Invitrogen (Carlsbad, USA) and the same amount of Pep 18 in a $1 \mathrm{mM} \mathrm{Ca}^{2+}$ buffer $\left(1 \mathrm{mM} \mathrm{CaCl}_{2}, 100 \mathrm{~mm} \mathrm{KCl}, 30 \mathrm{~mm}\right.$ MOPS, $\mathrm{pH}$ 7.2) was used. At a concentration of $0 \mu \mathrm{M}$, the absorption maximum is shown at $496 \mathrm{~nm}$ and at a $\mathrm{Ca}^{2+}$ concentration of $1 \mathrm{~mm}$, two maxima are found at $416 \mathrm{~nm}$ and $491 \mathrm{~nm}$. These can be clearly assigned to the fluorophore and indicates a $\mathrm{Ca}^{2+}$ dependency (see Chapter 4.2.3). In addition, the normalised excitation spectra of Pep 18 are given in Figure 4.37. Thus, the emission intensities at $\lambda_{\mathrm{Em}}=544 \mathrm{~nm}$ were recorded during a $\mathrm{Ca}^{2+}$ titration. Due to variations of the sensor concentration during the titration, a normalisation of the data was performed. Therefore, the excitation at $\lambda_{\mathrm{Ex}}=300 \mathrm{~nm}$ was used as $\mathrm{F}_{\min }$ and $\mathrm{F}_{\max }$ was taken out of the recorded excitation spectra. Although, the excitation spectra are comparable to the absorption spectra, the quality of the data hindered a precise determination of the maxima. 
a)

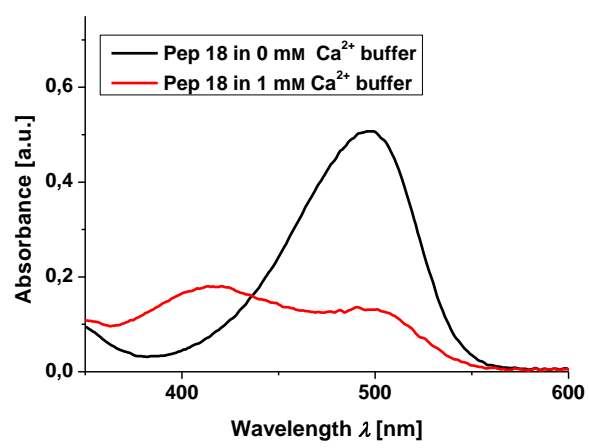

b)

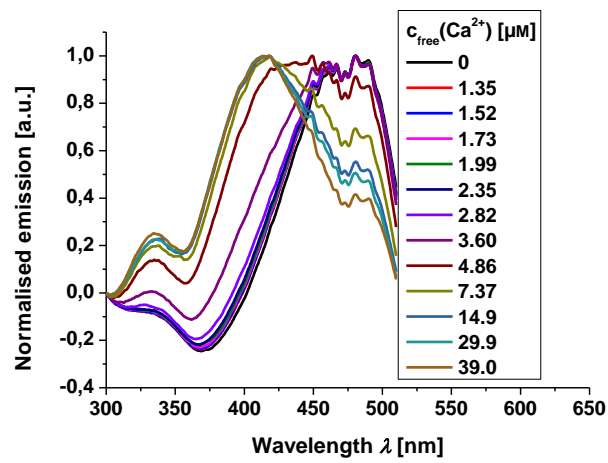

Figure 4.37 a) UV/Vis absorption spectra of Pep 18 in Zero Free $\mathrm{Ca}^{2+}$ buffer (10 mM K2EGTA, $100 \mathrm{mM} \mathrm{KCl}$, $30 \mathrm{mM}$ MOPS, pH 7.2, black curve) from INVITROGEN (Carlsbad, USA) and in a $1 \mathrm{mM} \mathrm{Ca} \mathrm{Caffer}^{2+} \mathrm{bum} \mathrm{CaCl}_{2}$, $100 \mathrm{~mm} \mathrm{KCl,} 30 \mathrm{~mm}$ MOPS, pH 7.2, red curve) $(T=\mathrm{RT})$. b) Excitation spectra of Pep 18 were recorded during a $\mathrm{Ca}^{2+}$ titration. The normalised emission intensities at $\lambda_{\mathrm{Em}}=544 \mathrm{~nm}$ were plotted against the excitation wavelengths.

The investigation of the $\mathrm{Ca}^{2+}$ dependent fluorescence of Pep 18 was carried out using a measuring kit with a range of $0 \mu \mathrm{M}$ to $39 \mu \mathrm{M}$ free $\mathrm{Ca}^{2+}$ (see Table 6.9 in Chapter 6.3) and two buffers were prepared with certain quantities of $\mathrm{CaCl}_{2}\left(100.0 \mu \mathrm{M}\right.$ or $2000.0 \mu \mathrm{M} \mathrm{CaCl}_{2}$, $100 \mathrm{~mm} \mathrm{KCl}, 30 \mathrm{~mm}$ MOPS, $\mathrm{pH}$ 7.2). For this purpose, the fluorescence was measured at an emission of $544 \mathrm{~nm}$ and the extinction was carried out at $416 \mathrm{~nm}$ and $496 \mathrm{~nm}$. The ratio $\mathrm{F}_{416 \mathrm{~nm}}^{544 \mathrm{~nm}} / \mathrm{F}_{496 \mathrm{~nm}}^{544 \mathrm{~nm}}$ with a presumed amount of fluorophore $(\sim 2 \mu \mathrm{M})$ as a function of the free $\mathrm{Ca}^{2+}$ concentration results in the graph shown in Figure 4.38. The exact concentration of the peptide could not be determined because the extinction coefficient given in the literature relate to a derivative of the used $\mathrm{Ca}^{2+}$-sensor. Changes due to the modification and the attachment to a peptide were excluded, which is, however, negligible because of the ratiometric consideration.

a)

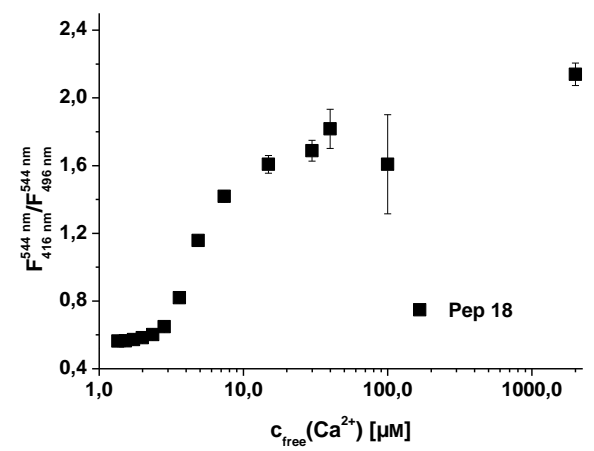

b)

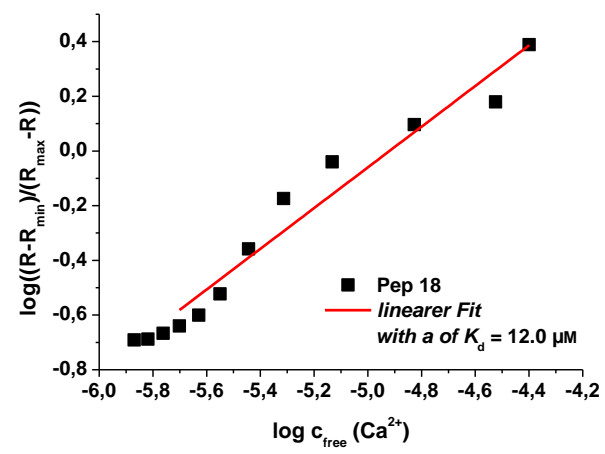

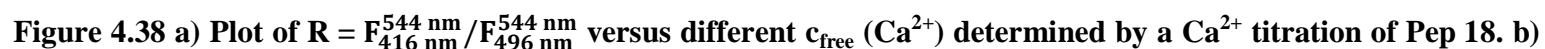
Linearisation of the $\mathrm{Ca}^{2+}$ titration of Pep 18. The graph of $\log \left(\mathbf{R}-\mathbf{R}_{\min }\right) /\left(\mathbf{R}_{\max }-\mathbf{R}\right)$ versus $\log c_{\text {free }}\left(C^{2+}\right)$ was plotted and a linear fit was performed in the range of $1.99 \mu \mathrm{M}$ to $39.0 \mu \mathrm{M}$ free $\mathrm{Ca}^{2+}$. As $\mathrm{R}_{\text {min }}$ the ratio of $0 \mu \mathrm{M} \mathrm{Ca}^{2+}$ was used and as $\mathbf{R}_{\text {max }}$ the ratio at $2000.0 \mu \mathrm{M}$ free $\mathrm{Ca}^{2+}$. 
The dissociation constant $\left(K_{\mathrm{d}}\right)$ was determined by the linearisation of the measurements which is shown in Figure 4.38. This application resulted in a value of $12.0 \mu \mathrm{M}$ for the $K_{\mathrm{d}}$ of

\section{Pep 18.}

The selectivity of the peptide-bound sensor Pep 18 towards $\mathrm{Ca}^{2+}$ and other divalent ions $\left(\mathrm{Mg}^{2+}\right.$ and $\left.\mathrm{Zn}^{2+}\right)$ was tested referring to the work of GRAF with a variation of metal ion concentrations. ${ }^{[274]}$ Especially, $\mathrm{Mg}^{2+}$ is of interest because it occurs in increased concentrations in the cytoplasm. ${ }^{[293]}$ The following measurements were performed in EGTA free buffer (100 mM KCl, $30 \mathrm{~mm}$ MOPS, pH 7.2) and the metal ion adjustment was performed by adding a certain volume of a $0.5 \mathrm{M}$ aqueous solution of the respective metal ion. The individual emission maxima $\left(F_{416}^{544 \mathrm{~nm}}\right.$ and $\left.F_{496}^{544 \mathrm{~nm}}\right)$ were measured and the resulting fluorescence ratio $F_{416 \mathrm{~nm}}^{544 \mathrm{~nm}} / \mathrm{F}_{496 \mathrm{~nm}}^{544 \mathrm{~nm}}$ was calculated. As a reference point for the ratio $\mathrm{F}_{416 \mathrm{~nm}}^{544 \mathrm{~nm}} / \mathrm{F}_{496 \mathrm{~nm}}^{544 \mathrm{~nm}}$, a presumed amount $(\sim 2 \mu \mathrm{M})$ of Pep 18 in EGTA free buffer (100 mM KCl, $30 \mathrm{~mm}$ MOPS, $\mathrm{pH} 7.2)$ was used $\left(\mathrm{F}_{416 \mathrm{~nm}}^{544 \mathrm{~nm}} / \mathrm{F}_{496}^{544 \mathrm{~nm}}=1.11 \pm 0.22, \mathrm{n}=15\right)$.

a)

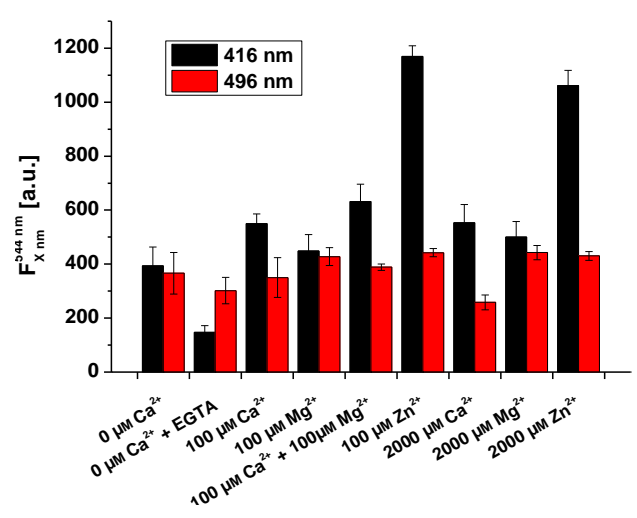

b)

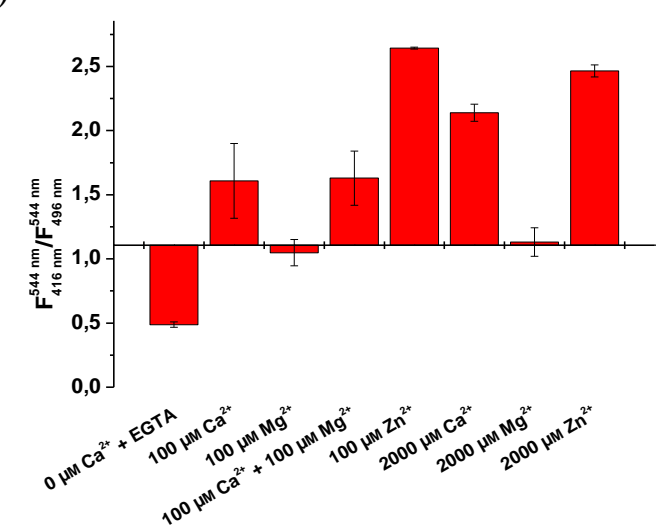

Figure 4.39 a) Fluorescence intensity of a constant amount Pep 18 in solutions with different ion concentration of $\mathrm{Ca}^{2+}, \mathrm{Mg}^{2+}$ and $\mathrm{Zn}^{2+}(\mathrm{n}=3)$. b) Calculated fluorescence ratio $\mathrm{F}_{416 \mathrm{~nm}}^{544} / \mathrm{F}_{496}^{544 \mathrm{~nm}}$ based on the measured fluorescence intensities. The reference point for the ratio $F_{416}^{544 \mathrm{~nm}} / F_{496}^{544} \mathrm{~nm}$ was determined by Pep 18 in EGTA free buffer (100 mM KCl, 30 mM MOPS, pH 7.2).

The variance of the reference point of Pep 18 in EGTA free buffer $(100 \mathrm{~mm} \mathrm{KCl}, 30 \mathrm{mM}$ MOPS, pH 7.2) was compared to Pep 18 in a Zero Free $\mathrm{Ca}^{2+}$ buffer $\left(10 \mathrm{mM} \mathrm{K} \mathrm{K}_{2} \mathrm{EGTA}\right.$, $100 \mathrm{~mm} \mathrm{KCl}, 30 \mathrm{~mm}$ MOPS, $\mathrm{pH} 7.2$ ). Here, a decrease in these $F_{416}^{544 \mathrm{~nm}} / F_{496 \mathrm{~nm}}^{544 \mathrm{~nm}}$ ratios of 0.62 was observed. This suggests that despite HPLC purification, cations were bound to the sensor. However, further ions $\left(\mathrm{Ca}^{2+}, \mathrm{Mg}^{2+}, \mathrm{Zn}^{2+}\right)$ were tested and an influence on the selectivity measurements was not considered. This assumption is supported by a different

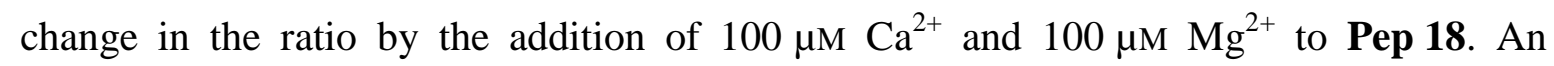
increase of the fluorescence ratio of 0.50 was observed after adding $100 \mu \mathrm{M} \mathrm{Ca}^{2+}$ and a decrease of 0.06 after adding $100 \mu \mathrm{M} \mathrm{Mg}^{2+}$. The ratio change after adding $100 \mu \mathrm{M} \mathrm{Mg}^{2+}$ is 
in the range of the error of the measurements $(n=3)$. A similar behaviour was observed after adding $2000 \mu \mathrm{M} \mathrm{Ca}^{2+}$ and $2000 \mu \mathrm{M} \mathrm{Mg}^{2+}$ to Pep 18 in EGTA free buffer. In contrast, the addition of $\mathrm{Zn}^{2+}(100 \mu \mathrm{M}$ and $2000 \mu \mathrm{M})$ to Pep 18 shows a strong increase in the fluorescence wavelength intensity (see Figure 4.39 a) and a strong increase of the ratio $\mathrm{F}_{416 \mathrm{~nm}}^{544 \mathrm{~nm}} / \mathrm{F}_{496 \mathrm{~nm}}^{544 \mathrm{~nm}}$ (see Figure $4.39 \mathrm{~b}$ ).

In addition to the ion selectivity measurements of Pep 18 with $\mathrm{Ca}^{2+}, \mathrm{Mg}^{2+}$ and $\mathrm{Zn}^{2+}$, the quenching influence of ions was investigated. Studies with Fura-2 showed quenching by the presents of $\mathrm{Co}^{2+}, \mathrm{Ni}^{2+}$ and $\mathrm{Mn}^{2+} \cdot{ }^{[270]}$ It is assumed that the paramagnetism of the ions induces a intersystem crossing, which lead to a decrease in the fluorescence. However, the quenching behaviour of different $\mathrm{Ni}^{2+}$ concentration towards Pep 18 in EGTA free buffer (100 mM KCl, $30 \mathrm{~mm}$ MOPS, $\mathrm{pH} 7.2)$ is shown in Figure 4.40. Therefore, similar to the selectivity measurements the following quenching measurements was performed in EGTA free buffer (100 mM KCl, $30 \mathrm{mM}$ MOPS, $\mathrm{pH}$ 7.2) and by adding a certain volume of a $0.5 \mathrm{M}$ aqueous solution of $\mathrm{NiCl}_{2}$.

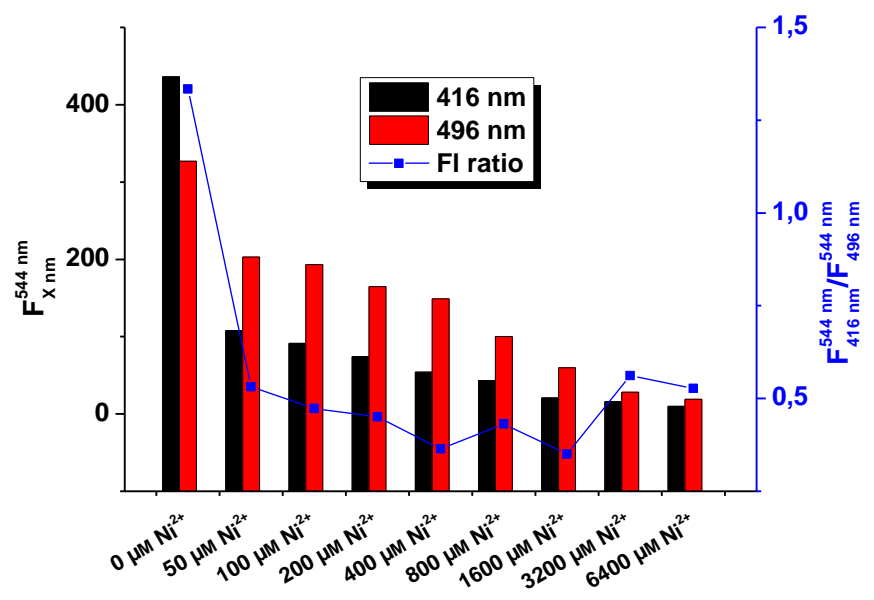

Figure 4.40 Exemplary presentation of the quenching effect of $\mathrm{Ni}^{2+}$ on Pep 18. The fluorescence intensity $\mathrm{F}_{416}^{544 \mathrm{~nm}}$ (black bars) and $F_{496}^{544} \mathrm{~nm}$ (red bars) and the fluorescence ratio $F_{416}^{544 \mathrm{~nm}} / F_{496}^{544 \mathrm{~nm}}$ (blue values) is plotted against different $\mathrm{Ni}^{2+}$ concentrations.

The peptide Pep 18 showed a decrease in the fluorescence intensities $F_{416}^{544 \mathrm{~nm}}$ and $F_{496}^{544 \mathrm{~nm}}$ with increasing $\mathrm{Ni}^{2+}$ concentrations. The $\mathrm{F}_{416 \mathrm{~nm}}^{544 \mathrm{~nm}} / \mathrm{F}_{496 \mathrm{~nm}}^{544 \mathrm{~nm}}$ ratio does not differ after the first $\mathrm{Ni}^{2+}$ addition (see Figure 4.40 blue data). Based on these results, the influence of $5.0 \mathrm{mM}$ $\mathrm{Co}^{2+}, \mathrm{Ni}^{2+}$ and $\mathrm{Mn}^{2+}$ Pep 18 was tested (see Figure 4.41). Therefore, a solution of Pep 18 in EGTA free buffer (100 mM KCl, $30 \mathrm{mM}$ MOPS, pH 7.2) was mixed with $0.5 \mathrm{M}$ aqueous solution of the respective metal ion. For the measurement the fluorescence intensities $F_{416}^{544 \mathrm{~nm}}$ and $\mathrm{F}_{496 \mathrm{~nm}}^{544 \mathrm{~nm}}$ were recorded each $3 \mathrm{~s}$ and after $300 \mathrm{~s}$ the metal ion was added (see 
Figure 4.41 a). Additionally, the fluorescence ratio $F_{416}^{544 \mathrm{~nm}} / F_{496}^{544 \mathrm{~nm}}$ was calculated, which is shown in Figure $4.41 \mathrm{~b}$ ).

a)

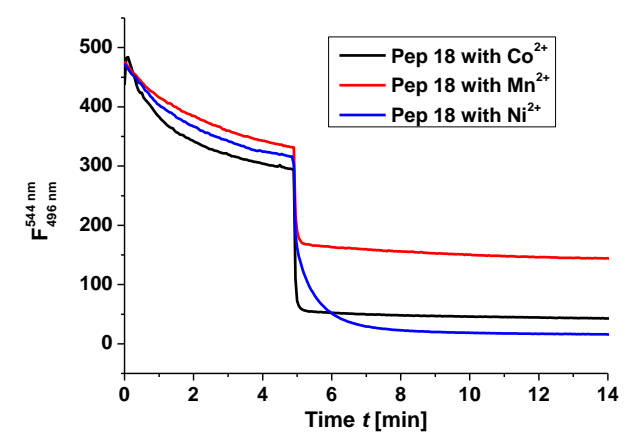

b)

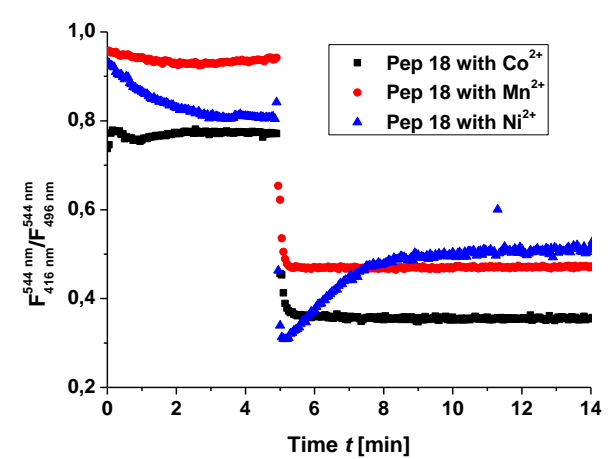

Figure 4.41 a) Measurement of the fluorescence intensity $\mathrm{F}_{496}^{544 \mathrm{~nm}}$ of Pep 18 in EGTA free buffer $(100 \mathrm{mM} \mathrm{KCl}$, $30 \mathrm{mM}$ MOPS, pH 7.2) and after $300 \mathrm{~s} 5.0 \mathrm{mM}$ of $\mathrm{Co}^{2+}, \mathrm{Ni}^{2+}$ or $\mathrm{Mn}^{2+}$ was added. b) Calculated fluorescence ratio $F_{416}^{544 \mathrm{~nm}} / F_{496}^{544 \mathrm{~nm}}$ of the partially in a) shown data.

It was found that all of the ions show a quenching effect at a concentration of $5.0 \mathrm{mM}$ at Pep 18. In Table 4.4 the percentage fluorescence decrease of Pep 18 at $F_{416}^{544 \mathrm{~nm}}$ and $\mathrm{F}_{496 \mathrm{~nm}}^{544 \mathrm{~nm}}$ after the addition (801 $\mathrm{s}$ ) is shown and as $100 \%$ the fluorescence intensities before the ion addition (294 s) was chosen. Additionally, the fluorescence ratio $F_{416}^{544 \mathrm{~nm}} / F_{496}^{544 \mathrm{~nm}}$ was calculated. It is noticeable that the quenching effect is not of equal amount for both wavelengths. This may be due to the mentioned effect of previous ion binding at Pep $\mathbf{1 8}$ (Figure 4.39). Nevertheless, a clear quenching effect can be seen, for example with a residual fluorescence of $5 \%$ after the addition of $5.0 \mathrm{mM} \mathrm{Ni}^{2+}$. In contrast to $\mathrm{Co}^{2+}$ and $\mathrm{Mn}^{2+}$ the fluorescence ratio $\mathrm{F}_{416 \mathrm{~nm}}^{544 \mathrm{~nm}} / \mathrm{F}_{496 \mathrm{~nm}}^{544 \mathrm{~nm}}$ shows no immediate stabilisation after the $\mathrm{Ni}^{2+}$ addition and only after $200 \mathrm{~s}$ such a stabilisation can be observed.

Table 4.4 Percentage fluorescence decrease of Pep 18 in EGTA free buffer (100 mM KCl, $30 \mathrm{~mm}$ MOPS, pH 7.2) at $F_{416}^{544} \mathrm{~nm}$ and $F_{496 \mathrm{~nm}}^{544}$ after the addition $(801 \mathrm{~s})$ and the calculated fluorescence ratio $F_{416 \mathrm{~nm}}^{544} / F_{496}^{544 \mathrm{~nm}}$ before (294 s) and after (801 s) the ion addition.

\begin{tabular}{|c|c|c|c|c|}
\hline & \multirow{2}{*}{$\mathrm{F}_{416 \mathrm{~nm}}^{544 \mathrm{~nm}}[\%]$} & \multirow{2}{*}{$F_{496 \mathrm{~nm}}^{544 \mathrm{~nm}}[\%]$} & \multicolumn{2}{|c|}{$F_{416 ~ n m}^{544} / F_{496 ~ n m}^{544 ~ n m}$} \\
\hline & & & $294 \mathrm{~s}$ & $801 \mathrm{~s}$ \\
\hline $\mathbf{N i}^{2+}$ & 3.28 & 5.37 & 0.841 & 0.515 \\
\hline $\mathrm{Mn}^{2+}$ & 21.9 & 43.6 & 0.941 & 0.474 \\
\hline $\mathrm{Co}^{2+}$ & 6.73 & 14.7 & 0.771 & 0.352 \\
\hline
\end{tabular}

In summary, the successful incorporation of a $\mathrm{Ca}^{2+}$-sensor into a $\beta$-peptide could be performed by SPPS. This water soluble peptide showed a $K_{\mathrm{d}}$ of $12.0 \mu \mathrm{M}$ for $\mathrm{Ca}^{2+}$ and a 
high $\mathrm{Ca}^{2+}$ selectivity in the presence of $\mathrm{Mg}^{2+}$. Moreover, it is possible to extinguish the fluorescence of the sensor by $\mathrm{Co}^{2+}, \mathrm{Ni}^{2+}$ and $\mathrm{Mn}^{2+}$.

\section{Synthesis and modification of an acid-labile $\mathrm{Ca}^{2+}$-sensor based on MOBHA and incorporation by SPPS to a water soluble $\beta$-peptide}

The sensor developed above shows a high $K_{\mathrm{d}}$ value compared to other known sensors (see Figure 4.31). However, the $\mathrm{Ca}^{2+}$ concentration close to membrane can be much higher (see Chapter 4.2.2). Therefore, the development of less affine sensors is necessary and a $\mathrm{Ca}^{2+}$-sensor comparable to $\mathbf{3 8}$ was synthesised based on the MOBHA chelator. This synthesis was inspired by the $\mathrm{Ca}^{2+}$-sensor Calcium Green FIAsH developed by Tour et al. (see Figure 4.42). ${ }^{[269]}$

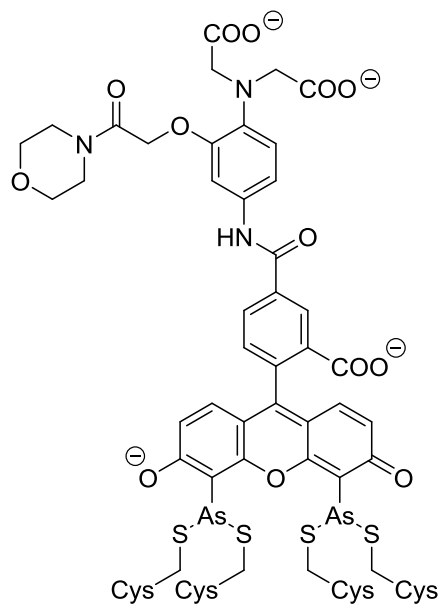

\section{Calcium Green FLAsH ${ }^{[269]}$} $K_{\mathrm{d}}=50-100 \mu \mathrm{M}$

Figure 4.42 Chemical structure and $K_{d}$ of Calcium Green FLAsH.

In order to obtain a $\mathrm{Ca}^{2+}$-sensor with a MOBHA-based chelator, the phenolic alcohol of $\mathbf{1 2}$ was deprotonated with $\mathrm{K}_{2} \mathrm{CO}_{3}$, followed by a nucleophilic substitution reaction with methyl bromoacetate to yield compound 39. Analogous to the BAPTA-based $\mathrm{Ca}^{2+}$-sensors, the nitro compound 39 was converted to the cycloamide compound $\mathbf{4 0}$ by BÉCHAMP reduction with cyclisation (seeScheme 4.11). ${ }^{[279,281,294,295]}$

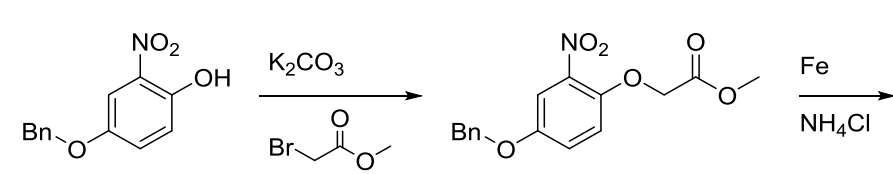

12

20

39<smiles>O=C1COc2ccc(OCc3ccccc3)cc2N1</smiles>

40

Scheme 4.11 Synthetic rout for the preparation of the protected amide 40 .

In contrast to the diamine compound 19 (see Chapter 4.2.5), a ring closing reaction occurred in the BÉCHAMP reduction of compound 39. ${ }^{[279,281,294,295]}$ 
The amide compound $\mathbf{4 0}$ was protected with di-tert-butyl dicarbonate to obtain the Bocprotected product 41. In a ring opening reaction with morpholine 28, isobutly chloroformate and triethylamine the Boc-protected aniline $\mathbf{4 2}$ was obtained (see Scheme 4.12).

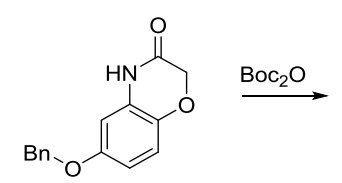

40

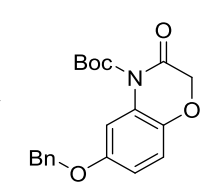

41

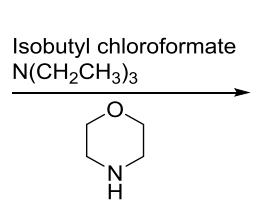

28

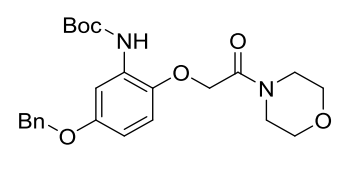

42

Scheme 4.12 Synthetic for the Boc-protected aniline 42.

The Boc-deprotection of $\mathbf{4 2}$ was performed with TFA, $\mathrm{H}_{2} \mathrm{O}$ and TIS as well as the subsequent reaction with tert-butyl bromoacetate gave product $\mathbf{4 3}$ and the following syntheses were analogous to $\mathbf{3 5} \mathbf{- 3 8}$ (seeScheme 4.13). An aldehyde group was introduced to the tert-butyl protected compound 43 with $\mathrm{POCl}_{3}$ and pyridine in DMF to yield the product 44. Subsequently, a Pd catalysed debenzylation, of $\mathbf{4 3}$ was performed to obtain the $o$-hydroxybenzaldehyde 45. Afterwards, the $o$-hydroxybenzaldehyde 45 was converted with 2-benzothiazoleacetonitrile 34 by a KNOEVENAGEL condensation to the tert-butyl protected MOBHA-based $\mathrm{Ca}^{2+}$-sensor $7 .^{[283,284]}$ The functionalisation of 7 was carried out by a nitrogen substitution with $\beta$-alanine hydrochloride 29 (see Scheme 4.13).

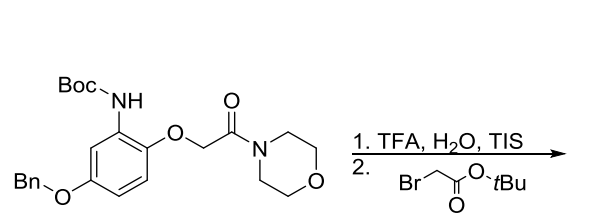

42

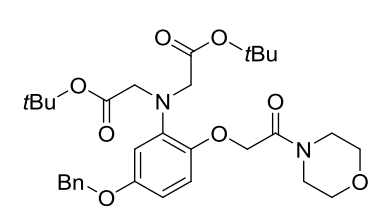

43

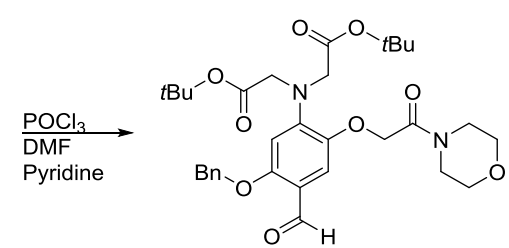

44

$\mathrm{H}_{2}$

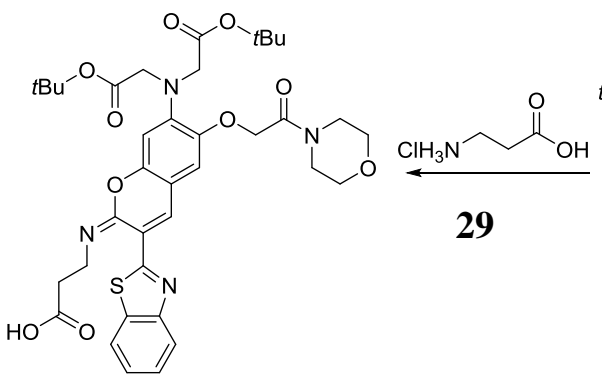

46

7

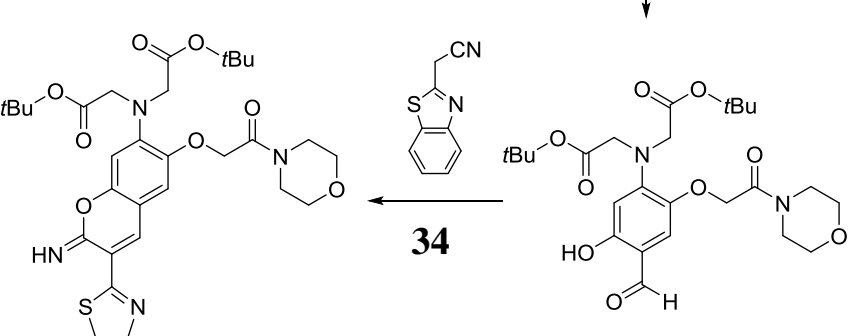

45

Scheme 4.13 Synthetic overview for the tert-butyl protected $\mathrm{Ca}^{2+}$-sensor 46 starting from the Boc-protected aniline 42. 
Analogous to 38, the first characterisation of the peptide-bound $\mathrm{Ca}^{2+}$-sensor 46 was performed with an alternating sequence of D- $\beta^{3}-h V a l$ and D- $\beta^{3}-h L y s$ (see Figure 4.43). This sequence allowed the observation of the peptide-bound $\mathrm{Ca}^{2+}$-sensor Pep 19 in aqueous medium without peptide aggregation or lipid-peptide interactions. Inspired by coupling conditions of the acid-labile $\mathrm{Ca}^{2+}$-sensor 38, the fluorophore 46 was coupled microwave-supported with PyAOP/HOAt/DIPEA. Therefore, the fluorophore (5.00 eq), PyAOP (10.00 eq) and HOAt (5.00 eq) was dissolved in DMF $(15 \mathrm{~mL} / \mathrm{mmol}$ based on resin-bound peptide) and subsequently DIPEA (15.00 eq) was added in three fractions to decrease heat development. This reaction mixture was added to the resin and if required DMF was added until the resin was completely covered with organic solvent. The fluorophore was coupled microwave-supported for $2 \mathrm{~h}$ to the resin-bound peptide $(20 \mathrm{~W}$, $2 \mathrm{~h}, 65^{\circ} \mathrm{C}$ (up to $10{ }^{\circ} \mathrm{C}$ overshoot).

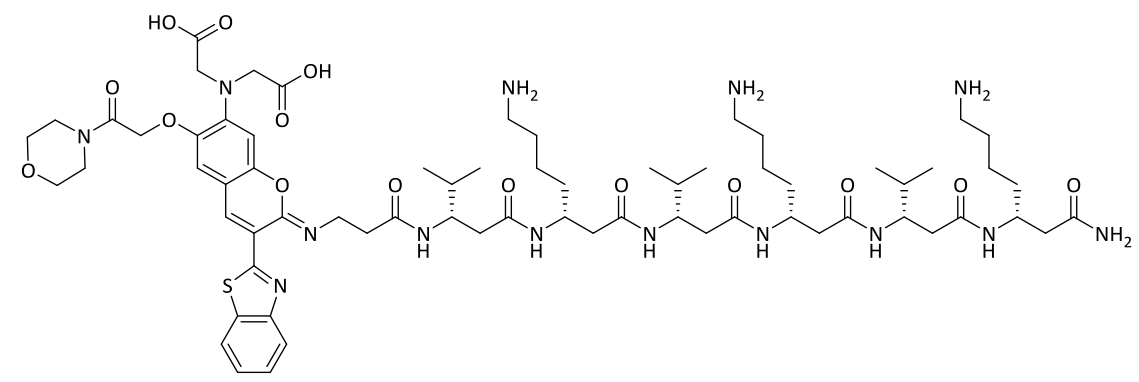

Figure 4.43 Sequence of peptide Pep 19.

\section{Pep 19}

Subsequent to the microwave-supported SPPS, the peptide was cleaved off with TFA/TIS (95:5) equally to Pep 18. This cleavage mixture was used to prevent a conversion of iminocoumarin to coumarin by water (see Scheme 4.9). The cleaved peptide was analysed by HPLC followed by mass spectrometric analysis. For this, the dried crude peptide was dissolved in water and examined with a gradient $30 \rightarrow 100 \% \mathrm{~B}\left(\mathrm{~A}: \mathrm{H}_{2} \mathrm{O}+0.1 \%\right.$ TFA and $\mathrm{B}: \mathrm{MeOH}+0.1 \% \mathrm{TFA}$ ). The HPLC chromatogram (see Figure 4.44) displays one peak with a retention time $t_{\mathrm{R}}=14.2$ min with an increasing absorption at $215 \mathrm{~nm}$, which is the specific wavelength of the peptide bond, and an increasing absorption at $480 \mathrm{~nm}$, which is the specific wavelength of the fluorophore. 
a)

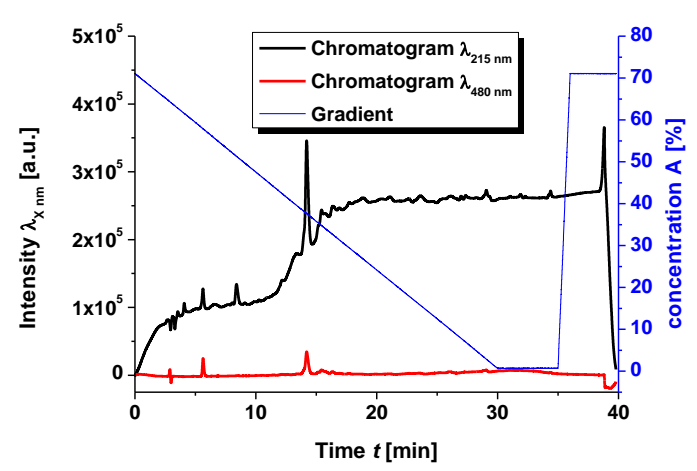

b)

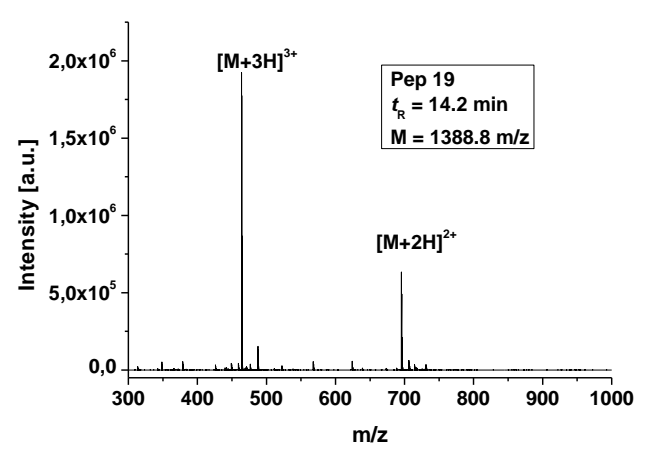

Figure 4.44 a) HPLC chromatogram of the crude peptide Pep 19. Absorption was recorded at $215 \mathrm{~nm}$ and $480 \mathrm{~nm}$. Analytical HPLC was performed using a gradient $30 \rightarrow 100 \% \mathrm{~B}\left(\mathrm{~A}: \mathrm{H}_{2} \mathrm{O}+0.1 \%\right.$ TFA and B: $\mathrm{MeOH}+$ $0.1 \%$ TFA) in $30 \mathrm{~min}$, flow $1.0 \mathrm{~mL} / \mathrm{min}$ and $25{ }^{\circ} \mathrm{C}$. The sample was dissolved in $100 \% \mathrm{H}_{2} \mathrm{O}$. b) ESI mass spectra of Pep 19 at $t_{\mathrm{R}}=14.2 \mathrm{~min}$.

MS analysis of the signal (see Figure 4.44) revealed in the peak at $t_{\mathrm{R}}=14.2 \mathrm{~min}$ is the desired product with a mass of $1388.8 \mathrm{~g} / \mathrm{mol}$. In contrast to Pep 18 no signal with a decreased mass by $58 \mathrm{~m} / \mathrm{z}$ was observed.

Following the successful synthesis of Pep 19 by SPPS, the different spectroscopic properties were investigated. As described in Chapter 4.2.3, the sensor presented here is a ratiometric sensor. The UV/Vis spectrum shows a change in the absorption band with increasing $\mathrm{Ca}^{2+}$ concentration. In Figure 4.45, the absorption curve of Pep 19 is depicted for $0 \mu \mathrm{M} \mathrm{Ca}$ and for $1 \mathrm{mM} \mathrm{Ca}^{2+}$. Therefore, a consistent amount of Pep 19 in Zero Free $\mathrm{Ca}^{2+}$ buffer (10 mM EGTA, $100 \mathrm{mM} \mathrm{KCl}, 30 \mathrm{mM}$ MOPS, pH 7.2) and in a $500 \mathrm{mM} \mathrm{Ca}^{2+}$ buffer (500 mM CaCl $2,100 \mathrm{mM} \mathrm{KCl,} 30 \mathrm{~mm}$ MOPS, pH 7.2) was used.

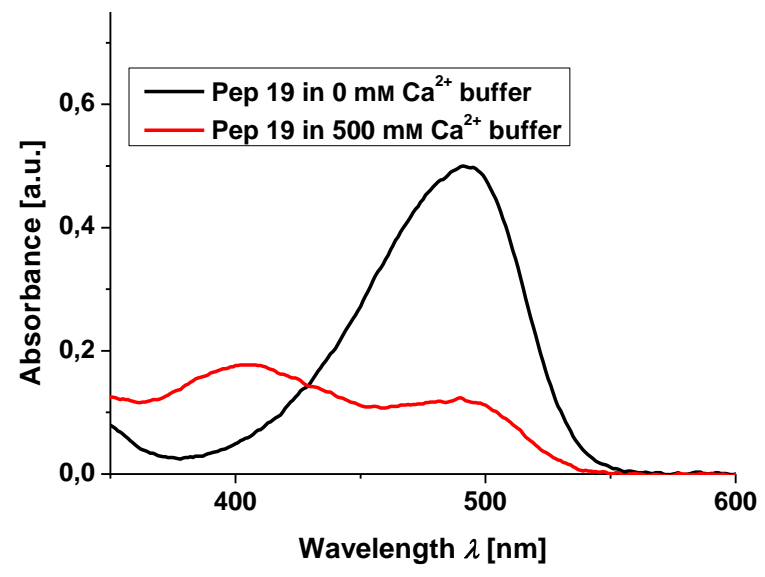

Figure 4.45 UV/Vis absorption spectra of Pep 19 in Zero Free $\mathrm{Ca}^{2+}$ buffer (10 mM K $\mathrm{K}_{2}$ ETA, $100 \mathrm{mM}$ KCl, $30 \mathrm{mM}$ MOPS, pH 7.2, black curve) from INviTROGEN (Carlsbad, USA) and in a $500 \mathrm{mM} \mathrm{Ca}{ }^{2+}$ buffer $\left(500 \mathrm{mM} \mathrm{CaCl}_{2}\right.$, $100 \mathrm{~mm} \mathrm{KCl,} 30 \mathrm{mM}$ MOPS, pH 7.2, red curve) $(T=\mathrm{RT})$.

At a concentration of $0 \mu \mathrm{M}$, the absorption maximum is shown at $489 \mathrm{~nm}$ and at a $\mathrm{Ca}^{2+}$ concentration of $500 \mathrm{mM}$ two maxima are found at $404 \mathrm{~nm}$ and $490 \mathrm{~nm}$ (normalised 
absorption spectra in Appendix C). The investigation of the $\mathrm{Ca}^{2+}$ dependent fluorescence of Pep $19(\sim 15 \mu \mathrm{M})$ was carried out with a measuring kit with a range of $0 \mu \mathrm{M}$ to $39 \mu \mathrm{M}$ free $\mathrm{Ca}^{2+}$ (see Table 6.9 in Chapter 6.3) and with prepared buffers with certain quantities of $\mathrm{CaCl}_{2}\left(0.0156-1000 \mathrm{mM} \mathrm{CaCl}_{2}, 100 \mathrm{mM} \mathrm{KCl}, 30 \mathrm{mM}\right.$ MOPS, $\mathrm{pH}$ 7.2). For this purpose, the fluorescence was measured at an emission of $539 \mathrm{~nm}$ and the extinction was carried out at $405 \mathrm{~nm}$ or $488 \mathrm{~nm}$ based on the maxima in the normalised absorption. The ratio $\mathrm{F}_{405 \mathrm{~nm}}^{539 \mathrm{~nm}} / \mathrm{F}_{488 \mathrm{~nm}}^{539 \mathrm{~nm}}$ with a presumed amount of fluorophore $(\sim 15 \mu \mathrm{M})$ as a function of the free $\mathrm{Ca}^{2+}$ concentration results in the graph shown in Figure 4.46. The exact concentration of the peptide could not be determined because the extinction coefficient given in the literature relates to a derivative of the used $\mathrm{Ca}^{2+}$-sensor. Changes due to the modification and attachment to a peptide were excluded, which is, however, negligible because of the ratiometric consideration.

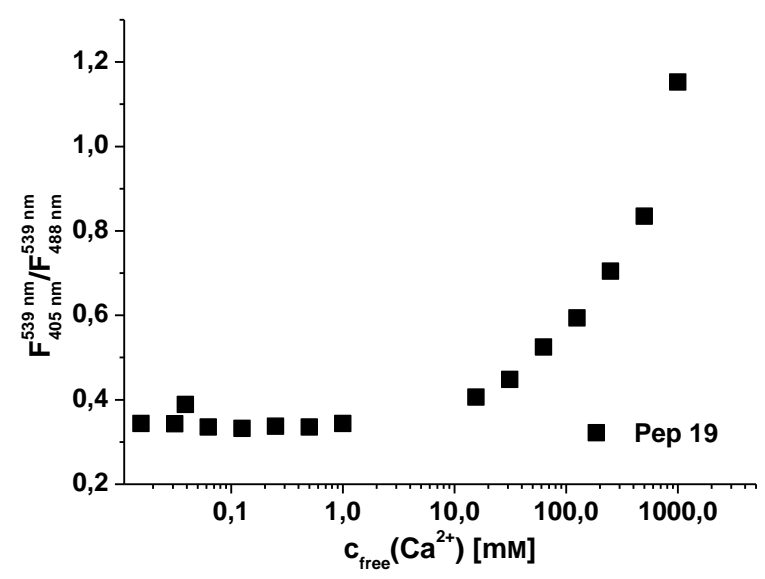

Figure 4.46 Plot of $R=F_{405}^{539} \mathrm{~nm} / F_{488}^{539} \mathrm{~nm}$ versus different $c_{\text {free }}\left(\mathrm{Ca}^{2+}\right)$ determined by a $\mathrm{Ca}^{2+}$ titration of Pep 19.

In contrast to the Pep $18 \mathrm{Ca}^{2+}$ titration, no complete saturation of Pep 19 was observed with the prepared buffer. Due to this result, the determination of dissociation constant $\left(K_{\mathrm{d}}\right)$ was not possible and the selectivity of the peptide-bound sensor Pep 19 towards other divalent ions $\left(\mathrm{Mg}^{2+}\right.$ and $\left.\mathrm{Zn}^{2+}\right)$ was not tested. Additionally, the exact $\mathrm{Ca}^{2+}$ concentration of the used buffers were not determined after preparation, for example by a $\mathrm{Ca}^{2+}$ electrode. Due to this, the concentration showed no comparable accuracy compared to the EGTA buffered system. Before further studies will be performed, a suitable method for identifying the $\mathrm{Ca}^{2+}$ concentration has needs to be found.

\section{Synthesis of transmembrane peptide labelled with tert-butyl protected $\mathrm{Ca}^{2+}$-sensor}

The different $\mathrm{Ca}^{2+}$ binding behaviour of the SPPS-compatible $\mathrm{Ca}^{2+}$-sensors $\mathbf{3 8}$ and $\mathbf{4 6}$ leads to further usage of 38, although in the analyses of Pep 18 side products were observed. 
Therefore, the $\mathrm{Ca}^{2+}$-sensor $\mathbf{3 8}$ was coupled to the transmembrane unit developed by RosT (see Figure 4.47). ${ }^{[11,38]}$ Analogous to the coupling conditions described in Chapter 4.1.4, the fluorophore 38 (3.00 eq), PyAOP (6.00 eq) and HOAt (3.00 eq) were dissolved in DMF $(15 \mathrm{~mL} / \mathrm{mmol}$ based on resin-bound peptide) and DIPEA (6.00 eq) was added subsequently in three fractions to decrease heat development. This reaction mixture was added to the resin and DMF was added if required until the resin was completely covered with organic solvent. The fluorophore was coupled microwave-supported for $2 \mathrm{~h}$ to the resin-bound peptide ( $20 \mathrm{~W}, 2 \mathrm{~h}, 65^{\circ} \mathrm{C}$ (up to $10{ }^{\circ} \mathrm{C}$ overshoot).

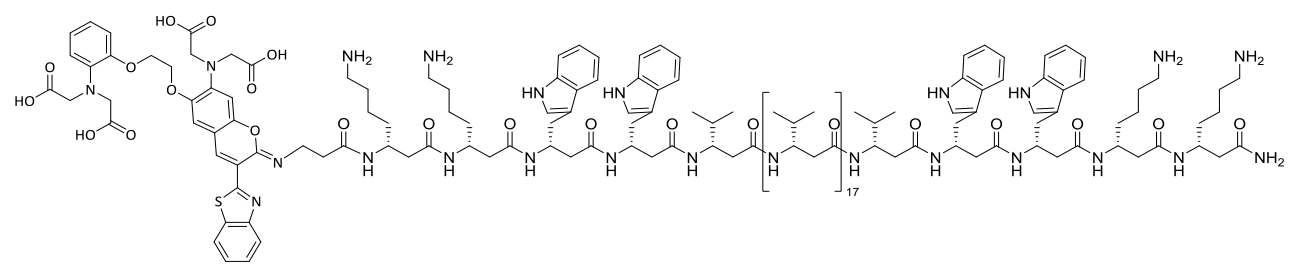

Figure 4.47 Sequence of peptide Pep 20.

Pep 20

The cleaved Pep 20 (TFA/TIS (95:5)) was freshly dissolved for each HPLC run in HFIP and examined with a gradient $90 \rightarrow 100 \% \mathrm{~B}\left(\mathrm{~A}: \mathrm{H}_{2} \mathrm{O}+0.1 \%\right.$ TFA $+0.1 \%$ HFIP and $\mathrm{B}$ : $\mathrm{MeOH}+0.1 \% \mathrm{TFA}+0.1 \%$ HFIP). Contrary to other HPLC purification of hydrophobic transmembrane units at elevated temperature (Pep 13, Pep 14), the purification of Pep 20 was performed at RT. Elevated temperatures facilitate the hydrolysis of iminocoumarin to coumarin. ${ }^{[283]}$ In Figure 4.48 the HPLC chromatogram of Pep 20 with a high peak at $t_{\mathrm{R}}=14.4 \mathrm{~min}$ is shown.

a)

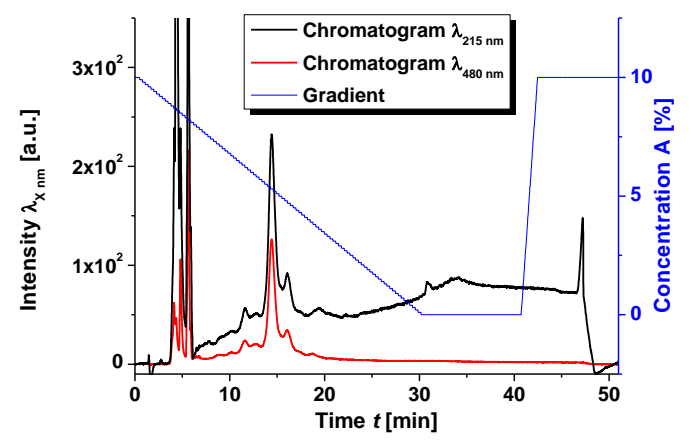

b)

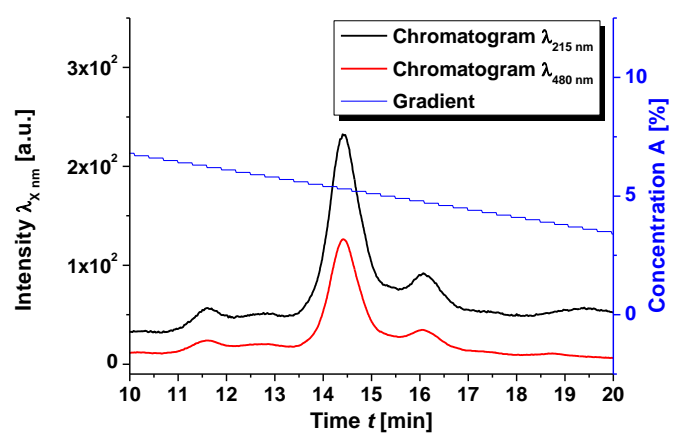

Figure 4.48 Absorption was recorded at $215 \mathrm{~nm}$ and $480 \mathrm{~nm}$. a) Preparative HPLC was performed using a gradient $90 \rightarrow 100 \% \mathrm{~B}\left(\mathrm{~A}: \mathrm{H}_{2} \mathrm{O}+0.1 \% \mathrm{TFA}+0.1 \%\right.$ HFIP and B: $\left.\mathrm{MeOH}+0.1 \% \mathrm{TFA}+0.1 \% \mathrm{HFIP}\right)$ in $30 \mathrm{~min}$, flow $10.0 \mathrm{~mL} / \mathrm{min}$ and RT. The sample was freshly dissolved in $100 \%$ HFIP. b) Zoom of the HPLC chromatogram in a range of $10 \mathrm{~min}$ to $20 \mathrm{~min}$. The recorded peak at $t_{\mathrm{R}}=14.4$ appears as a symmetric peak.

The main peak was collected in two different fractions from $13.8 \mathrm{~min}-14.4 \mathrm{~min}$ (fraction A) and from $14.4 \mathrm{~min}-15.0 \mathrm{~min}$ (fraction $\mathrm{B}$ ). The resulting mass spectrometric analyses 
are given in Figure 4.49. In the spectrometric data of the collected fraction A, the target peptide Pep 20, the defunctionalised peptide-bound $\mathrm{Ca}^{2+}$-sensor (see Scheme 4.10) and the transmembrane unit with an attached $\beta$-alanine was found.

a)

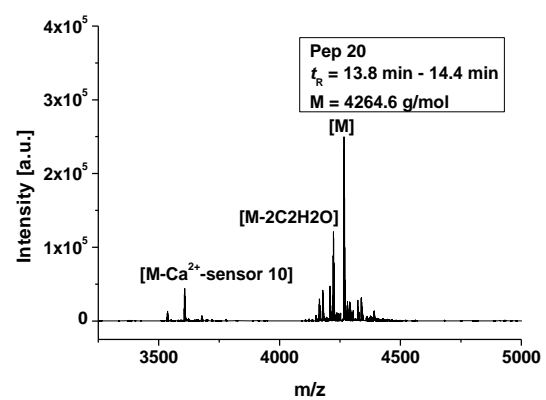

b)

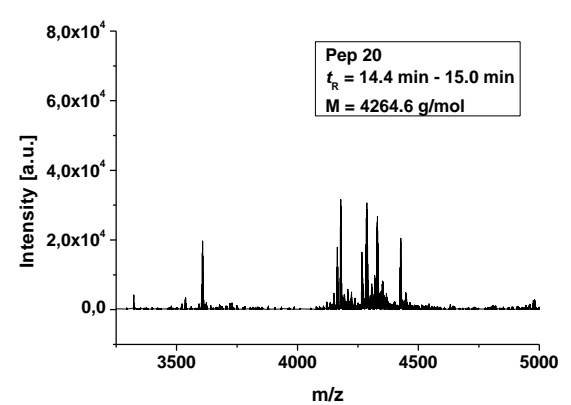

Figure 4.49 Deconvoluted ESI mass spectra of Pep 20 at $t_{\mathrm{R}}=\mathbf{1 4 . 4} \mathrm{min}$. The peak was separately collected in a range of a) $13.8 \mathrm{~min}-14.4 \mathrm{~min}$ and b) $14.4 \mathrm{~min}-15.0 \mathrm{~min}$.

In previous studies of Pep 18 and Pep 19 in this work, the cleavage of the peptide and the attached $\mathrm{Ca}^{2+}$-sensor was not observed. This side product can be formed by the conversion of the iminocoumarin to coumarin (see Scheme 4.14). This mechanism leads to the elongation of the transmembrane unit by the $\beta$-alanine linker of the sensor $\left[\mathrm{M}-\mathrm{Ca}^{2+}\right.$-sensor 10]. Compared with Pep 18, the reason for the side product origin is unclear. In the collected fraction B multiple signals which cannot be allocated are seen.
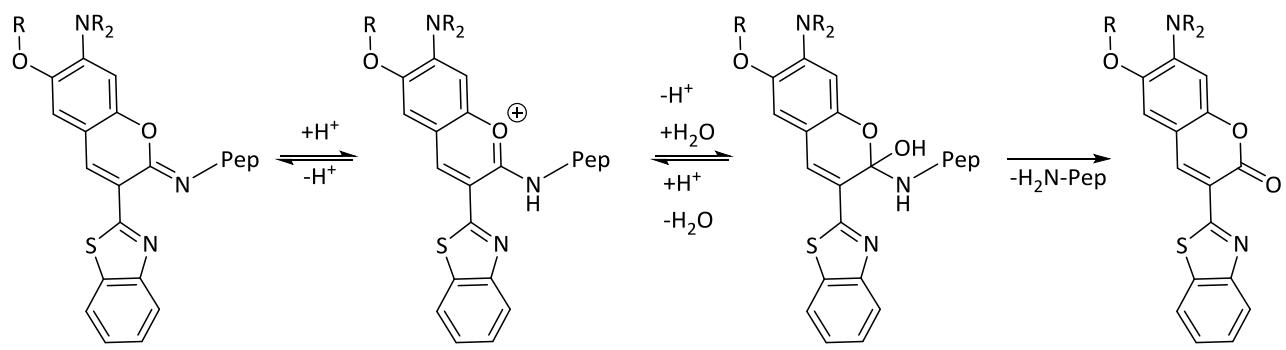

Scheme 4.14 Formation of the transmembrane unit elongated by $\beta$-alanine seen in mass spectrometric analyses. ${ }^{[283]}$

Nevertheless, in the fraction A the target product was clearly identified. This allows the usage of a transmembrane $\beta$-peptide functionalised with a $\mathrm{Ca}^{2+}$-sensor in further studies, whereby the negligibility of the both side products has to be clarified.

\subsubsection{Conclusion and outlook}

Cellular $\mathrm{Ca}^{2+}$ concentration plays an important role in many processes in the cell, especially for the transmission of nerve signals (see Chapter 4.2.1). Probes for examining this $\mathrm{Ca}^{2+}$ concentration are the basis for the influence of $\mathrm{Ca}^{2+}$ in biological processes. 
In this work it was possible to synthesise and modify a literature known $\mathrm{Ca}^{2+}$-sensors with different functionalities. ${ }^{[37,278]}$ The functionalisation with $\beta$-alanine enabled it to apply the $\mathrm{Ca}^{2+}$-sensors 38 and 46 in microwave-supported SPPS. Comparable with the literature known $\mathrm{Ca}^{2+}$-sensors BIIC and BTIC, the reaction yields were similar. ${ }^{[37,278,279]}$ As an exception, the reduction with $\mathrm{Fe}$ and $\mathrm{Pd} / \mathrm{C}$ did not achieve the expected controllability. ${ }^{[37,278]}$ In the $\mathrm{Fe}$ mediated reduction, the product was completely decomposed several times. Therefore, consideration should be given to other conditions such as Se based reductions. ${ }^{[296]}$ To obtain high yields in the $\mathrm{Pd} / \mathrm{C}$ mediated reduction, it is necessary to monitor the reactions conditions and the reaction time. Based on the successful synthesis of the unmodified $\mathrm{Ca}^{2+}$-sensors $\mathbf{8}$, it was possible to incorporate differed functional groups that can be used as attachment point for peptides $\left(-\mathrm{NH}_{2}\right.$ and $-\mathrm{SH}$ groups). The attachment of $\beta$-alanine to $\mathbf{8}$ enabled the development and synthesis of the BAPTA-based $\mathrm{Ca}^{2+}$-sensors 38 and MOBHA-based 46. Therefore, the tert-butyl protected $\mathrm{Ca}^{2+}$-sensor derivates were used to combine the $\mathrm{Ca}^{2+}$-sensor with the Fmoc-based SPPS. ${ }^{[297,298]}$ Apart from the literature known fluorophores, no variation was performed. However, varying the fluorophore can also provide a wide range of modification. ${ }^{[37,278]}$ In Figure 4.50 an overview of the different modification positions is given.
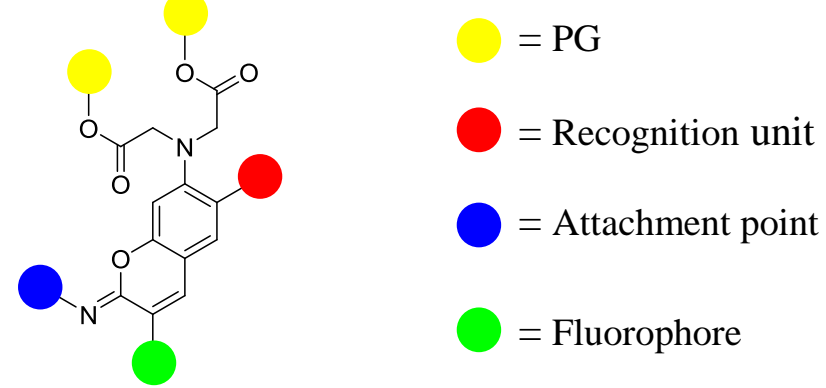

Figure 4.50 Overview of the variation possibility in the used iminocoumarin system.

The modifications shown in this work were established in known synthesis routes, in particular the attachment of the fluorophore and the attachment point for functional groups. This modifiability was possible because the fluorophore and the attachment point were incorporated in the last synthesis steps with little synthetic effort.

The $\mathrm{Ca}^{2+}$-sensors $(\mathbf{3 8}, \mathbf{4 6})$ were coupled to a hydrophilic $\beta$-peptide motif, which resulted in two peptides (Pep 18, Pep 19). Despite the liability of the iminocoumarin basic structure, these peptides have shown no formation of coumarin in the cleavage mixture and the HPLC solvents at RT. However, Pep 18 showed a partial decomposition, which was 
suggested as the release of a chelating carboxylic acid from the BAPTA unit. In contrast to the hydrophilic peptide, the coumarin formation was observed in mass spectrometric analyses of Pep 20, which is the $\beta$-peptide transmembrane unit developed by RosT functionalised with the $\mathrm{Ca}^{2+}$-sensor 38. ${ }^{[11,38]}$ Nevertheless, the results received in ion titration showed that the BAPTA-based $\mathrm{Ca}^{2+}$-sensor $\mathbf{3 8}$ is a good design for upcoming $\mathrm{Ca}^{2+}$-sensors in peptide chemistry and in biomolecular systems. The MOBHA-based $\mathrm{Ca}^{2+}$-sensor 46 in Pep 19 showed a higher stability during the peptide cleavage compared to Pep 18. Additionally, the $K_{\mathrm{d}}$ of Pep 19 is estimated higher than the measured $K_{\mathrm{d}}$ of Pep 18. However, further studies have to be performed with verified $\mathrm{Ca}^{2+}$ concentration, for example by a $\mathrm{Ca}^{2+}$ sensitive electrode. Especially for the low affinity $\mathrm{Ca}^{2+}$-sensors, the MOBHA-based $\mathrm{Ca}^{2+}$-sensor offers a wide range of applications, but does not show the modifiability of the BAPTA motif. Despite, the small number of amino acids and the attachment of the $\mathrm{Ca}^{2+}$-sensors, the $\beta$-peptides Pep 18 and Pep 19 show a 14-helix (see Appendix C).

The transmembrane $\beta$-peptide based Pep 20 can enable the observation of near-membrane $\mathrm{Ca}^{2+}$ concentration and can help to find out the $\mathrm{Ca}^{2+}$ distribution in the electrostatic double layer. SinN et al. postulated, based on measurements with ion selective electrodes and isothermal titration calorimetry (ITC), that the $\mathrm{Ca}^{2+}$ binding is regardless of the lipid type. ${ }^{[299]}$ The formal higher $\mathrm{Ca}^{2+}$ binding on negative charged membranes depends on the electrostatic enrichment in the electrostatic double layer. ${ }^{[299]}$ Therefore, first measurements with Pep 20 were performed in DOPC and DOPC/DOPS (90:10) vesicles (100 nm) and a $\mathrm{P} / \mathrm{L}$ ratio of $1: 1000$ in $\mathrm{Ca}^{2+}$ free or $\mathrm{Ca}^{2+}$ buffer (162 mM sucrose, $10 \mathrm{mM}$ HEPSE, pH 7.2 (adjust with free base arginine), optional with $\left.2 \mathrm{mM} \mathrm{CaCl}_{2}\right) \cdot{ }^{[299-301]}$ However, unspecific changes in the fluorescence were observed, which were not connected to the peptide-bound sensor, but the reason is unclear so far. Therefore, experiments with buffered $\mathrm{Ca}^{2+}$ concentration were performed. According to ScHWAMBORn, LUV with Pep 20 was prepared with a lipid composition of DOPC/biotin-cap-DOPE (99.6:0.4) and a P/L ratio of $1: 200 .^{[30,203]}$ One probe of LUV was prepared with Zero Free $C a^{2+}$ buffer $(10 \mathrm{mM}$ $\mathrm{K}_{2}$ EGTA, $100 \mathrm{mM} \mathrm{KCl,} 30 \mathrm{mM}$ MOPS, $\mathrm{pH}$ 7.2) whereas the other one was prepared with $39 \mu \mathrm{M}$ Free $\mathrm{Ca}^{2+}$ buffer (10 mM CaEGTA, $100 \mathrm{mM} \mathrm{KCl,} 30 \mathrm{~mm}$ MOPS, pH 7.2). Both vesicle types were immobilised according to SCHWAMBORN, excited by two lasers $(405 \mathrm{~nm}$ and $488 \mathrm{~nm}$ ) and detected in a range of $505 \mathrm{~nm}-602 \mathrm{~nm} \cdot{ }^{[30,203]}$ However, the vesicles prepared in Zero Free $\mathrm{Ca}^{2+}$ buffer showed compared to the LUV prepared in $39 \mu \mathrm{M}$ Free 
$\mathrm{Ca}^{2+}$ buffer a intensive fluorescence. The LUV prepared with Zero Free $\mathrm{Ca}^{2+}$ buffer showed aggregation effects on the functionalised surface, but nevertheless, a localisation of the peptide to the vesicles is assumed (see Figure 4.51). With the same probes fluorescence measurements were performed according to the measurements of Pep 18 and Pep 19. These measurements showed a change in the fluorescence ratio $F_{416}^{544 \mathrm{~nm}} / F_{496 \mathrm{~nm}}^{544 \mathrm{~nm}}(0 \mu \mathrm{M}$ $\mathrm{Ca}^{2+}=0.84 ; 39 \mu \mathrm{M}=1.41$ ) and a shift of the fluorescence maxima from $\sim 544 \mathrm{~nm}$ (Pep 18) to $\sim 530 \mathrm{~nm}$ (Pep 20, average maximum values of $0 \mu \mathrm{M}$ and $39 \mu \mathrm{M}$ free $\mathrm{Ca}^{2+}$ ). The possibilities of $\beta$-peptide anchored $\mathrm{Ca}^{2+}$-sensors (e.g. Pep 20) must be investigated further.

a)

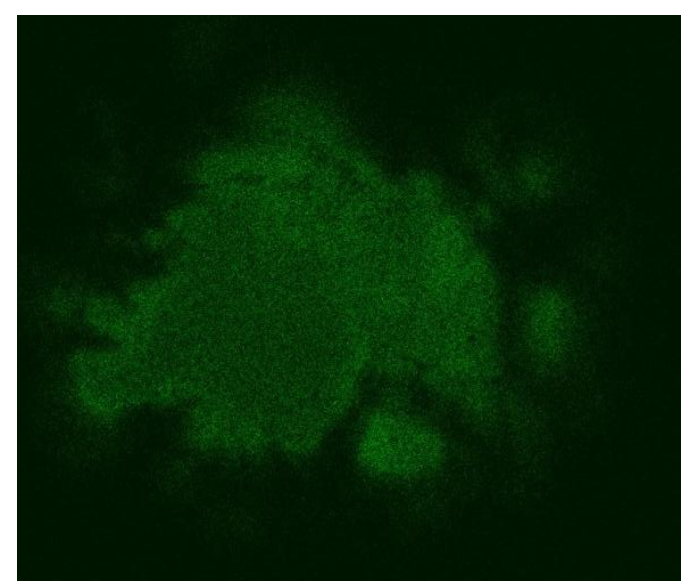

b)

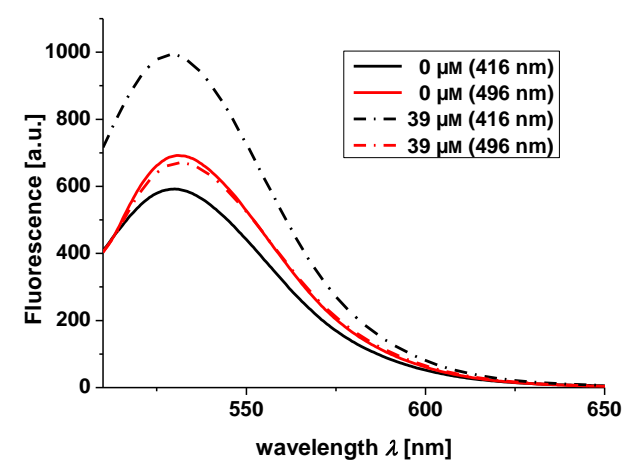

Figure 4.51 a) Fluorecence micrograph of immobilized proteoliposomes (DOPC/biotin-cap-DOPE (99.6:0.4); P/L ratio of 1:200) ${ }^{[30,203]} \mathrm{LUV}$ was prepared in Zero Free $\mathrm{Ca}^{2+}$ buffer (10 mм K2 $\mathrm{EGTA}, 100 \mathrm{mM} \mathrm{KCl,} 30 \mathrm{mM}$ MOPS, pH 7.2) and the detection was performed in a range of $505 \mathrm{~nm}-602 \mathrm{~nm}(e x .=405 \mathrm{~nm}$ and $488 \mathrm{~nm})$. b) Fluorescence spectra of LUV prepared in Zero Free $\mathrm{Ca}^{2+}$ buffer (10 mM K K EGTA, $100 \mathrm{~mm} \mathrm{KCl}, 30 \mathrm{~mm}$ MOPS, pH 7.2) and in $39 \mu$ M Free $\mathrm{Ca}^{2+}$ buffer (10 mM CaEGTA, $100 \mathrm{~mm}$ KCl, $30 \mathrm{~mm}$ MOPS, pH 7.2).

In future studies, new applications for the developed system and new design approaches for $\mathrm{Ca}^{2+}$-sensors should be examined. The developed $\mathrm{Ca}^{2+}$-sensors enable the labelling of $\beta$-peptide membrane anchors, as shown in this work, or additionally the usage of lipids as membrane anchors. In addition to the above described $\mathrm{Ca}^{2+} /$ membrane interaction, the $\mathrm{Ca}^{2+}$ movement across the surface can be interesting for cellular processes. Inspired by the experimental setup of the POHL group (see Chapter 4.1.5) a caged $\mathrm{Ca}^{2+}$ can be incorporated into the membrane and released by photoinduced cleavage. This system can also be used to imitate the influx of a $\mathrm{Ca}^{2+}$ channel. Although no membrane anchored caged $\mathrm{Ca}^{2+}$ is known NP-EGTA and DMNP-EDTA are known water-soluble structures. The modification with alkyl chains can be led to a suitable structure for the experimental setup of the PoHL group. In addition, a new design approach for the $\mathrm{Ca}^{2+}$-sensors can lead to an improvement of the sensor properties. Modification of the BAPTA unit enables the adjustment of the 
$\mathrm{Ca}^{2+}$-sensor $K_{\mathrm{d}}$ value. GEE et al. were able to manipulate the $K_{\mathrm{d}}$ of Fluo-4 $(0.345 \mu \mathrm{M})$ by exchanging a methyl substituent with fluorine atom (Fluo-5F, $2.3 \mu \mathrm{M}$ ) or nitro group (Fluo-5N, $90 \mu \mathrm{M}) .{ }^{[302]}$ In addition to the configuration of the chelating unit, a modification of the fluorophore and the iminocoumarin is possible. In the work of RousSAKIS et al. ${ }^{\text {[297] }}$ the synthesis of a near-membrane $\mathrm{Ca}^{2+}$-sensor is given based on BIIC (see Figure 4.52).

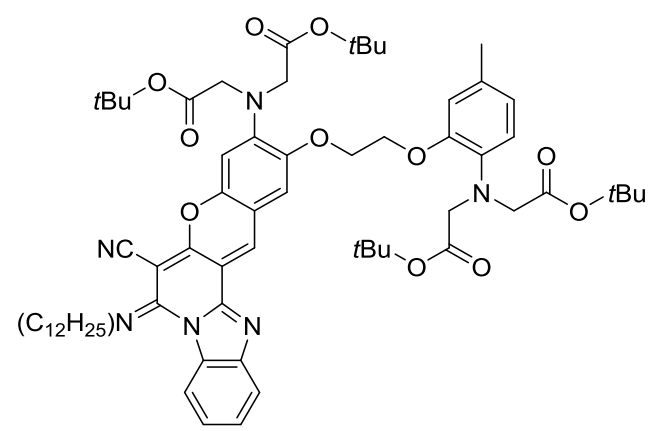

Figure 4.52 Chemical structure of C12-ICPBC developed by RouSSAKIS et al. ${ }^{[297]}$

The modification developed by ROUSSAKIS et al. shows the conversion of the 3(benzimidazolyl)-iminocoumarin (BIIC) to a chromeno[3', $\left.2^{\prime}: 3,4\right]$ pyrido[1,2a][1,3]-benzimidazole chromophore (ICPBC). ${ }^{[297]}$ The received fluorophore can also be modified by nitrogen substitution and does not show the instability against aqueous acid solutions of an iminocoumarin. However, ROUBINET et al. ${ }^{[288]}$ had converted an iminocoumarin with $\mathrm{BF}_{3} \times \mathrm{Et}_{2} \mathrm{O}$ into an boron(III) iminocoumarin, which is a boron-dipyrromethene (BODIPY) analogue. Furthermore, there are other $\mathrm{Ca}^{2+}$-sensors, which can be useful in the peptide modification. Tour et al. synthesised Calcium Green FLAsH as a $\mathrm{Ca}^{2+}$-sensor with a $K_{\mathrm{d}}$ of $50-100 \mu \mathrm{M}$ and a bis-ARSenic fluorescein. ${ }^{[269]}$ This sensor has shown a self-labelling at a tetracysteine tag. ${ }^{[118,269]}$ CALDWELL et al. synthesised also a low affinity ratiometric $\mathrm{Ca}^{2+}$ (NitroAzidoFuraRed) which was modified with an azide group. Based on this modification, it was possible to label a $N$-terminal modified protected amino acid with NitroAzidoFuraRed by copper-catalysed azide-alkyne cycloaddition. ${ }^{[279]}$ The synthesised $o$-hydroxybenzaldehyde in the presented work could be used for the synthesis of NitroAzidoFuraRed derivates, which lead to an alternative of the iminocoumarin system for peptide labelling $\mathrm{Ca}^{2+}$-sensors.

However, the presented $\mathrm{Ca}^{2+}$-sensors 38 and 46 show possible structures for the establishment of $\mathrm{Ca}^{2+}$-sensors in SPPS and a wide range of application in biochemical systems because of various attachment point modifications. 


\section{Conclusion}

A variety of cellular key processes is performed by peptides or proteins, which are localised at the cell membrane. ${ }^{[1]}$ The properties and the functions of such biomolecules are very complex, for this reason peptidomimetics were developed. ${ }^{[6]}$ Among other things, these peptidomimetics reduced the function of a peptide and helped to investigate basic properties. $^{[6]}$

In the present work $\beta$-peptides were synthesised as peptidomimetics for transmembrane helices. ${ }^{[1,9]}$ In addition to the development of automatic SPPS protocols for $\beta$-peptides, the secondary structure of the synthesised $\beta$-peptides, which is influenced by the choice of different amino acids was examined by CD spectroscopy. ${ }^{[14]}$ It was demonstrated that the branching of the side chain groups has an influence on the secondary structure, whereby it was possible to change a 12-helix (D- $\beta^{3}$-homoleucine) into a 14-helix (D- $\beta^{3}$-homoisoleucine, D- $\beta^{3}$-homovaline) in organic solvents as well as in lipid model systems (Pep 1 - Pep 8). ${ }^{[14]}$ Furthermore, forcing a 12-helix into a 14-helix by ring structure amino acids (ACHC) was successfully shown (Pep 9, Pep 10). ${ }^{[14]}$ The synthesised $\beta$-peptides were equipped with certain functionalities, which can be classified into sensors in the transmembrane region and in sensors in the intercellular region of the peptides (see Figure 5.1). On the one hand, the transmembrane sensors should work as tools for the observation of the peptide surrounding and the membrane-peptide interaction. On the other hand, the intercellular functionalities are sensors, which use the $\beta$-peptides as a transmembrane anchor and should help to investigate membrane surface processes (see Figure 5.1). The functionalisation of Pep 1 -Pep 10 with D- $\beta^{3}$-homotryptophan (D- $\beta^{3}-\mathrm{hTrp}$ ) enabled it to characterise the surrounding of the peptides with the help of tryptophan fluorescence. These measurements showed that an incorporation of the synthesised peptides Pep 1 - Pep 10 in the membrane was successful. ${ }^{[14]}$ To determine the exact position of the D- $\beta^{3}$-hTrp in the membrane, tryptophan fluorescence quenching based on DOXYL-labelled lipids was performed. The general tendency of the DOXYL based measurements is in line with preliminary considerations, although unknown lipid-peptide interactions lead to inconsistent data. ${ }^{[22-25]}$ 


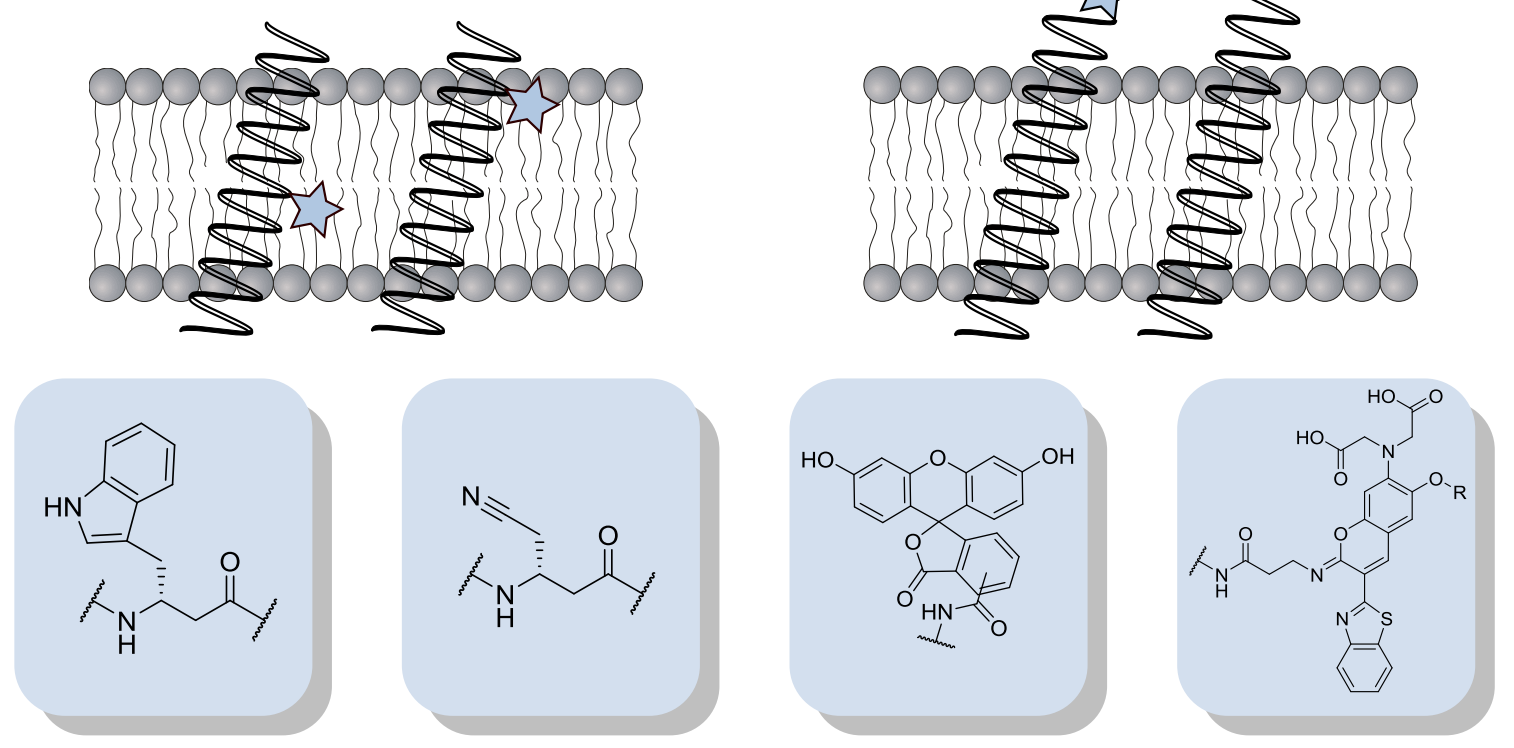

Figure 5.1 Overview of the performed functionalisation in this work and the location in the synthesised peptides.

In addition to fluorescence measurements, IR based spectroscopic methods are widespread in the observation of transmembrane peptides. ${ }^{[166-168]}$ Therefore, a potential IR active D- $\beta^{3}$-amino acid was successfully synthesised. SEIRA measurements performed by the HiLDEBRANDT group showed the peptide incorporation of Pep 11, though the nitrile typical vibration band was not observed. However, the developed IR active D- $\beta^{3}$-amino acid can be used in further studies with other measurement setups or other IR active D- $\beta^{3}$-amino acid can be synthesised on the basis of the presented synthesis route. These IR measurements are able to unravel further properties of transmembrane $\beta$-peptides.

The stable membrane position of the $\beta$-peptide unit, developed by Rost, ${ }^{[1,38]}$ enables the anchoring of sensors near the membrane surface. Also, the properties of the $\beta$-peptide make it possible to serve as molecular rulers for adjusting a defined membrane distance. ${ }^{[10]}$ This is possible by the extension of the peptide primary sequence. To test this possible application field, $\beta$-peptides were functionalised with $\mathrm{H}^{+}$(Pep 13, Pep 14) and $\mathrm{Ca}^{2+}$ sensors (Pep 18 -Pep 20). The $\beta$-peptides Pep 13 and Pep 14, were labelled with carboxyfluorescein to test the $\mathrm{pH}$ dependency in different distances to the membrane. Some studies presumed that the lipid membrane surface act as $\mathrm{H}^{+}$collector and, furthermore, that the $\mathrm{H}^{+}$moves across the membrane. ${ }^{[186,208]}$ Based on fluorescence titration no $\mathrm{p} K_{\mathrm{a}}$ change through the membrane-bounding of carboxyfluorescein, compared with literature known $\mathrm{pH}$ sensor titrations, was observed. ${ }^{[31,32]}$ Additionally, no significant change in the $\mathrm{p} K_{\mathrm{a}}$ was observed with the distance of the fluorophore to the membrane. Due 
to variable effects, these results may be unclear at the moment. Nevertheless, a $\mathrm{pH}$ dependency of the labelled transmembrane peptide was observed. In particular the interaction of the membrane surface and the peptide will be of interest. Therefore, measurements in cooperation with the POHL group and the ENDERLEIN group are still in progress.

$\mathrm{Ca}^{2+}$ is one of the most important second messengers and interacts with the membrane surface. ${ }^{[188,243]}$ These ion-membrane interactions increase the near-membrane $\mathrm{Ca}^{2+}$ concentration compared to the cellular concentration. ${ }^{[299]}$ A sensor for the near-membrane $\mathrm{Ca}^{2+}$ concentration has to fulfil certain requirements. On the one hand the $\mathrm{Ca}^{2+}$-sensor has to be anchored near the membrane and on the other side the $\mathrm{Ca}^{2+}$ affinity has to be lower than for the intercellular $\mathrm{Ca}^{2+}$ concentration. ${ }^{[264]}$ Among others, two SPPS capable $\mathrm{Ca}^{2+}$-sensor were developed and attached to water-soluble $\beta$-peptides and to the transmembrane unit, developed by RosT. ${ }^{[11,38]}$ These iminocoumarin-based $\mathrm{Ca}^{2+}$-sensors 38 and 46 were coupled to water-soluble peptides (Pep 18, Pep 19) to determine the binding properties without membrane influences. The $\mathrm{Ca}^{2+}$-sensor labelled peptide Pep 18 showed a side product after the cleavage from the resin, which was not observed in Pep 19. Despite the low number of amino acids and the attachment of the $\mathrm{Ca}^{2+}$-sensors, Pep 18 and Pep 19 showed a 14-helix. However, Pep 18 showed good binding properties for $\mathrm{Ca}^{2+}$ and insensitivity against $\mathrm{Mg}^{2+}$. Whereas Pep 19 showed a higher stability against the cleavage mixture and a lower affinity against $\mathrm{Ca}^{2+}$, which will result in a higher $K_{\mathrm{d}}$. The determination of the $K_{\mathrm{d}}$ from Pep 19 was not possible so far, because no buffered system can be used and a $\mathrm{Ca}^{2+}$ electrode was unavailable. Therefore, 38 was coupled to the literature known transmembrane $\beta$-peptide unit (Pep 20). ${ }^{[11,38]}$ Here, the incomplete purification showed, beside the known side product, an additional side product, which is a result of the conversion of iminocoumarin to coumarin. ${ }^{[283]}$ Nevertheless, the peptide synthesis was successful and first measurements showed a $\mathrm{Ca}^{2+}$ depending fluorescence of the peptide mixture incorporated in DOPC/biotin-cap-DOPE LUV. ${ }^{[30]}$ In further research projects the application of the synthesised $\mathrm{Ca}^{2+}$-sensors and the attachment to transmembrane $\beta$-peptides has to be studied.

To summarise, in this work a wide range of $\beta$-peptides has been synthesised. These synthesised peptides allowed, on the one hand, investigating properties of transmembrane $\beta$-peptides and on the other hand, investigating functionalised transmembrane $\beta$-peptides for membrane surface studies. The received results suggested that the transmembrane 
$\beta$-peptides provide a variable helix structure which lead to a wide range of design motifs. The anchoring of sensors by transmembrane $\beta$-peptides to measure near-membrane interactions should be subject of further researches and compared with other anchoring methods like lipids. 


\section{Experimental part}

\subsection{General}

\section{Solvents}

Technical solvents were partially distilled before use. All other solvents were at the highest grade available. Dry solvents were purchased from ACROS ORGANICS (Geel, Belgium) and stored over molecular sieves ( $4 \AA$ ). Solvents of analytical or HPLC grade were obtained from FLUKA (Taufkirchen, Germany), VWR INTERNATIONAL (Fontenay-sous-Bois, France), ACros Organics (Geel, Belgium) and SigMA-ALDRICH (Taufkirchen, Germany). Ultrapure water was obtained by using a water purification device SIMPLICITY (MILLIPORE, Bredford, UK) or ARIUM MINI lab water system by SARTORIUS (Göttingen, Germany).

\section{Reagent}

All reagents were of highest grade available and used as supplied. Amino acids, coupling reagents and resin for solid peptide synthesis were obtained from BACHEM (Bubendorf, Swzitzerland), GL BIOCHEM (Shanghai, China), IRIS BIOTECH (Marktredwitz, Germany), NOVABIOCHEM (Darmstadt, Germany) or VWR INTERNATIONAL (Fontenay-sous-Bois, France). Lipids were purchased from AVANTI POLAR LIPIDS (Alabama, USA). All other chemicals were purchased from ABCR (Karlsruhe, Germany), ACROS ORGANICs (Geel, Belgium), Alfar Aesar (Karlsruhe, Germany), ApPlCHEm (Darmstadt, Germany), CARL RоTH GMBH (Karlsruhe, Germany), FluKA (Taufkirchen, Germany), FluOROCHEM (Hadfield, UK), GrÜssing GmbH (Filsum, Germany), Merck (Darmstadt, Germany), RIEDEL-DE HAËN (Seelze, Germany), SIGMA-AlDRICH (Taufkirchen, Germany), TCI (Zwijndrecht, Belgium) and VWR INTERNATIONAL (Fontenay-sous-Bois, France).

\section{Reaction}

Air and/or water sensitive reactions were carried out under argon atmosphere (> $99.9 \%$ ) using standard SCHLENK technique. The inert gas was conducted through a drying tube and was dried over pumice/ $\mathrm{NaOH} /$ pumice. If necessary, the glass apparatus was heated with a heat gun under reduced pressure and flushed with argon $(3 \times)$. Manuel solid phase peptide synthesis was carried out in a syringe from Becton DICKINSON (Heidelberg, Deutschland) with inserted PE filter. The manual microwave-assisted reaction was performed in a DISCOVER microwave reaction cavity (CEM, Kamp-Lintfort, Germany). Here, the listed reaction temperature can be up to $10^{\circ} \mathrm{C}$ higher than listed. The automatic microwave-supported SPPS was performed in a Liberty Blue from CEM. 


\section{Storage}

The resins, building blocks, synthesised peptides and lipids were stored at $-20{ }^{\circ} \mathrm{C}$. The chemicals were thawed at RT before use.

\section{Freeze-drying}

Lyophilisation from water or dioxane solution was performed by using a CHRIST ALPHA-24 LYOPHILIZER (Osterode am Harz, Germany) equipped with a high vacuum pump. For probes with a total volume fewer than $2 \mathrm{~mL}$ an evacuable CHRIST RVC 2-18 CD plus centrifuge was used, which was connected to the lyophilisation device.

\subsection{Chromatography}

\section{Thin layer chromatography (TLC)}

Analytic TLC was performed on MERCK (Darmstadt, Germany) coated aluminium sheets of silica gel $60 \mathrm{~F}_{254}$ (layer thickness $0.25 \mathrm{~mm}$ ). The substance on the developed TLC plate was visualised under UV fluorescence at $254 \mathrm{~nm}$ or $366 \mathrm{~nm}$. Alternatively, the TLC plate was immersed into an appropriate reagent (Table 6.1) and subsequently heated.

\section{Table 6.1 Applied TLC stains.}

\begin{tabular}{|cc|}
\hline TLC stain - Functional group & Composition \\
\hline Ninhydrin stain & $0.30 \mathrm{~g}$ ninhydrin, $100 \mathrm{~mL} \mathrm{EtOH}$ \\
Free amino group & \\
\hline Cer/Molybdenum stain & $2.50 \mathrm{~g}$ ammonium molybdate tetrahydrate, \\
Various groups & $1.00 \mathrm{~g}$ cerium ammonium sulfate dehydrate, \\
& $10 \mathrm{~mL}$ sulfuric acid, $90 \mathrm{~mL}$ water \\
\hline Dinitrophenylhydrazine (DNP) & $12.00 \mathrm{~g} \mathrm{2,4-dinitrophenylhydrazine,}$ \\
Aldehyde groups & $60 \mathrm{~mL} 98 \%$ sulfuric acid, $80 \mathrm{~mL}$ water, \\
& $200 \mathrm{~mL} 95 \%$ ethanol \\
\hline
\end{tabular}

\section{Flash column chromatography}

For flash column chromatography silica gel $60 \mathrm{~F}_{254}(40-63 \mu \mathrm{m})$ from MERCK (Darmstadt, Germany) was used at $0.3-1.0$ bar. Columns were packed with wet silica gel (50100-fold weight excess) and the sample was given on the column absorbed onto silica gel, as a concentrated solution or as a solid matter.

\section{High performance liquid chromatography (HPLC)}

Reversed phase (RP)-HPLC analyse were performed on a device (Äkta Basic 900, pump type P 900, variable wavelength detector UV-900) of AMERSHAM PHARMACIA BIOTECH 
(Freiburg, Germany), if required equipped with a PHARMACIA LKB HPLC column oven 2155. Alternatively, analytic HPLC runs were performed by a HPLC system from JASCO (Tokio, Japan) equipped with an MD-2010 diode array detector, two PU-2085 semi-micro pumps, a CO-2060 column thermostat and an AS-2055 autosampler. The peptides were detected by UV-absorption at $215 \mathrm{~nm}, 254 \mathrm{~nm}, 280 \mathrm{~nm}$ and fluorophore specific wavelength (see Table 6.2).

Table 6.2 Used wavelength for detection of NBD, carboxyfluorescein and the synthesised $\mathrm{Ca}^{2+}$-sensors.

\begin{tabular}{|cc|}
\hline Fluorophore & Specific wavelength \\
\hline NBD & $464 \mathrm{~nm}$ \\
\hline Carboxyfluorescein & $480-492 \mathrm{~nm}$ \\
\hline $\mathbf{C a}^{2+}$-sensor 38/46 & $480 \mathrm{~nm}$ \\
\hline
\end{tabular}

The substances were analysed and purified with a linear gradient of a two-component system (solvent $\mathrm{A}=$ water phase, solvent $\mathrm{B}=$ organic phase). The following columns from MACHEREY-NAGEL (Düren, Germany) were used:

MN Nucleodur ${ }^{\circledR}$ 100-5-C18, $250 \mathrm{~mm} \times 4.6 \mathrm{~mm}, 5 \mu \mathrm{m}, 1 \mathrm{~mL} / \mathrm{min}$ (Analytic),

MN Nucleodur ${ }^{\circledR} 100-5-\mathrm{C} 18,250 \mathrm{~mm} \times 10 \mathrm{~mm}, 5$ m, $3 \mathrm{~mL} / \mathrm{min}$ (Semipreperative),

MN Nucleodur ${ }^{\circledR} 100-5-\mathrm{C} 18,250 \mathrm{~mm} \times 21 \mathrm{~mm}, 5 \mu \mathrm{m}, 10 \mathrm{~mL} / \mathrm{min}$ (Preperative).

\subsection{Characterisation}

\section{Nuclear magnetic resonance (NMR)}

${ }^{1} \mathrm{H}$ - and ${ }^{13} \mathrm{C}$-NMR spectra were recorded with a spectrometer (Unity 300, Mercury-Vx 300, VNMRS-300, Inova 500 or Inova 600) by VARIAN (Palo Alto, USA) or an av301 by BRUKER (Billerica, USA) spectrometer. The spectra were recorded at RT or $308.1 \mathrm{~K}$. Chemical shift $(\delta)$ are denoted in parts per million (ppm) downfield of TMS. The resonance of the residual protons of the deuterated solvents was used as internal standard. Abbreviations for multiplicities are: $\mathrm{s}$ (singlet), $\mathrm{d}$ (doublet), $\mathrm{t}$ (triplet), q (quartet), $m$ (multiplet), br (broad). Coupling constants ${ }^{n} J_{X, Y}$ are stated in Hertz (Hz), where $n$ is the order of coupling and $\mathrm{X}$ and $\mathrm{Y}$ the coupling partners. 


\section{Mass spectrometry (MS)}

Electron ionisation (EI) measurements were performed on an AccuTOF obtained from JEOL (Akishima, Japan). Electrospray-ionisation (ESI) and high resolution ESI (HR-MS (ESI)) spectra were obtained with a BRUKER device (maXis or MicrOTOF).

\section{UV/Vis spectroscopy}

$\mathrm{UV} / \mathrm{V}$ is spectrometry to determinate resin loading, peptide concentration and absorption spectra of $\mathrm{Ca}^{2+}$-sensor labelled $\beta$-peptides were measured by using a THERMO SCIENTIFIC (Waltham, USA) NanoDrop ND-2000c spectrophotometer. The concentration measurements of the peptides in organic solvents (MeOH/TFE) were performed in a $1 \mathrm{~cm}$ cuvette at $280 \mathrm{~nm}$. The $\mathrm{Ca}^{2+}$-sensor labelled $\beta$-peptides (Pep 18 and Pep 19) UV/Vis spectra in aqueous buffer were recorded with the micro-volume pedestal method or with a JASCO (Groß-Umstadt, Germany) V-650 UV-Visible Spectrophotometer. Quartz glass cuvettes Suprasil $^{\circledR}$ (QS) were used as cuvettes (Hellma, Müllheim, Germany). The concentration was calculated by using the LAMBERT-BEER LAW (Equation 6.1).

$$
\mathrm{c}\left[\frac{\mathrm{mol}}{\mathrm{L}}\right]=\frac{\mathrm{A}_{\lambda}}{\mathrm{N} \cdot \mathrm{d} \cdot \varepsilon_{\mathrm{Fl}}} \cdot \mathrm{f}
$$

$\mathrm{A}_{\lambda}=$ Absorption at specific wavelength $[\mathrm{nm}] \quad \mathrm{d}=$ pathlength $[\mathrm{cm}]$

$\mathrm{N}=$ number of fluorophore $\quad \varepsilon_{\mathrm{Fl}}=$ molar attenuation coefficient $\left[\mathrm{cm}^{-1} \mathrm{M}^{-1}\right]$

$\mathrm{f}=$ dilution factor $\quad \varepsilon_{280, \operatorname{Trp}}(\mathrm{MeOH})=5690 \mathrm{~cm}^{-1} \mathrm{M}^{-1}$

The following values ${ }^{[37]}$ were used for the concentration measurement of the synthesised $\mathrm{Ca}^{2+}$-sensors:

$$
\begin{array}{ll}
\mathrm{Ca}^{2+} \text {-free } & \varepsilon_{471}\left(\mathrm{H}_{2} \mathrm{O}\right)=17000 \mathrm{~cm}^{-1} \mathrm{M}^{-1}, \\
\mathrm{Ca}^{2+} \text {-bound } & \varepsilon_{421}\left(\mathrm{H}_{2} \mathrm{O}\right)=9000 \mathrm{~cm}^{-1} \mathrm{M}^{-1} .
\end{array}
$$

These values were used, although a shift in the maximum compared to the literature was observed. The concentration dependent measurements of Pep 14 in $\mathrm{MeOH}$ and the normalised absorption of Pep 19 were measured by using a JASCO (Groß-Umstadt, Germany) V-650 UV-Visible Spectrophotometer. The following settings have been selected: 
Table 6.3 UV/Vis settings for measurements of Pep 14 in MeOH with variation peptide concentration and UV/Vis settings for measurements of Pep 19 during a $\mathrm{Ca}^{2+}$ titration.

\begin{tabular}{|ccc|}
\hline & Pep 14 & Pep 19 \\
\hline Response & Fast & Medium \\
\hline Scan speed & $100 \mathrm{~nm} / \mathrm{min}$ & $200 \mathrm{~nm} / \mathrm{min}$ \\
\hline UV/Vis bandwidth & $0.5 \mathrm{~nm}$ & $2.0 \mathrm{~nm}$ \\
\hline Change source at & $350 \mathrm{~nm}$ & $330 \mathrm{~nm}$ \\
\hline Start/end & $600-250 \mathrm{~nm}$ & $600-300 \mathrm{~nm}$ \\
\hline Data interval & $0.5 \mathrm{~nm}$ & $0.2 \mathrm{~nm}$ \\
\hline Accumulation & 3 & 1 \\
\hline Cell length & $1 \mathrm{~cm}$ & $1 \mathrm{~cm}$ \\
\hline Temp & $25^{\circ} \mathrm{C}$ & $20^{\circ} \mathrm{C}$ \\
\hline
\end{tabular}

\section{Circular dichroism (CD) measurement}

The CD spectra were recorded on a J-1500 CD spectrometer (JASCO, Groß-Umstadt, Germany) equipped with a F250 thermostat (JulaBO, Seelbach, Germany). Therefore, the measurement chamber was flushed with nitrogen. The temperature has been varied for the different solvents and lipids $\left(\mathrm{H}_{2} \mathrm{O}, \mathrm{MeOH}\right.$, TFE, DOPC: $T=25^{\circ} \mathrm{C}$; DMPC: $T=30^{\circ} \mathrm{C}$; DPPC: $T=50{ }^{\circ} \mathrm{C}$ ). For the CD measurement normally a cuvette with a length of $0.1 \mathrm{~cm}$ was used (with the exception of the Pep 12), which was measured in a $0.01 \mathrm{~cm}$ cuvette. As cuvettes quartz glass cuvettes Suprasil $^{\circledR}$ (QS) were used (Hellma, Müllheim, Germany). Table 6.4 contains the measurement parameters for the $\mathrm{CD}$ measurements.

Table 6.4 Settings for measurements of CD spectra.

\begin{tabular}{|cc|}
\hline Photometric mod & CD spectra \\
\hline D.I.T. & $1.0 \mathrm{~s}$ \\
\hline Bandwidth & $1.0 \mathrm{~nm}$ \\
\hline Speed scan & $20-50 \mathrm{~nm} / \mathrm{min}$ \\
\hline Start/end & $250-180 \mathrm{~nm}$ \\
\hline Data pitch & $0.1-1.0 \mathrm{~nm}$ \\
\hline Accumulation & $5-50$ \\
\hline CD scale & $200 \mathrm{mdeg} / 1.0 \mathrm{dOD}$ \\
\hline Fl scale & $200 \mathrm{mdeg} / 1.0 \mathrm{dOD}$ \\
\hline
\end{tabular}

For the CD measurements, the peptides were solved in organic solvent $(300 \mu \mathrm{L}, \sim 50 \mu \mathrm{M})$ or undiluted vesicle suspension was prepared according to Chapter SOP 7. 
The spectra were corrected with a blank spectrum of solvent or LUV without peptides. The ellipticity $\Theta_{\mathrm{abs}}[\mathrm{mdeg}]$ was converted into the molar ellipticity $\Theta_{\mathrm{mol}}\left[\mathrm{mdeg} \mathrm{cm}^{2} \mathrm{dmol}^{-1}\right]$ with the Equation 6.2 of GREENFIELD. ${ }^{[303]}$

$$
\Theta_{\mathrm{mol}}=\frac{\Theta_{\mathrm{abs}}}{\mathrm{c} \cdot \mathrm{d}}
$$

$\Theta_{\mathrm{abs}}[\mathrm{mdeg}]=$ ellipticity

$\mathrm{c}[\mathrm{mol} / \mathrm{L}]=$ peptide concentration

$\mathrm{d}[\mathrm{mm}]=$ layer thickness

\section{Dynamic light scattering (DLS)}

The distribution of the prepared vesicles was obtained with a Zetasizer Nano S light scattering system (MALVERN INSTRUMENTS, Malvern, UK). As light source a $4 \mathrm{~mW}$ laser $(633 \mathrm{~nm})$ was used and the light scattering was detected in an angle of $173^{\circ}$. Data acquisition was performed at different temperature in a range of $20-30{ }^{\circ} \mathrm{C}$. A $1.5 \mathrm{~mL}$ or a $2.5 \mathrm{~mL}$ PS disposable cuvette (BRAND, Wertheim, Germany) was used. The measurement parameters were optimised by the device automatically.

\section{Fluorescence measurements \\ General}

Fluorescence measurements were performed on a J-1500 CD spectrometer (JASCO, GroßUmstadt, Germany) equipped with a monochromator FMO-427S/15 (JASCO, GroßUmstadt, Germany), on a FP-6200 (JASCO, Groß-Umstadt, Germany) or on a FP-8500 (JASCO, Groß-Umstadt, Germany). Each spectrometer was equipped with a F250 thermostat (JULABO, Seelbach, Germany). For the fluorescence measurements a $1.5 \mathrm{~mL}$ quartz glass cuvette Suprasil $^{\circledR}$ (QS) (HELlmA, Müllheim, Germany) or a $2.5 \mathrm{~mL}$ UVtransparent disposable cuvette (BRAND, Wertheim, Germany) was used. With exception of the $\mathrm{Ca}^{2+}$-sensor measurements $\left(\mathrm{Ca}^{2+}\right.$ titration measurments $)$, the fluorescence measurements were corrected by blank measurements of solvent or lipids without peptides. The solution was stirred at 500 r.p.m during the $p H$ fluorescence measurements, NBD distribution measurement and $\mathrm{Ca}^{2+}$-sensor fluorescence measurements. Furthermore, the corrected spectra were partially normalised with the following Equation 6.3: 


$$
A_{\text {nom }}=\frac{x-A_{\text {min }}}{A_{\max }-A_{\min }}
$$

$\mathrm{A}_{\text {min }}=$ Minimum fluorescence

$$
\mathrm{A}_{\max }=\text { Maximum fluorescence }
$$

$\mathrm{A}_{\text {nom }}=$ Normalized fluorescence

In the case of multiple fluorescence measurements, the mean value was calculated and displayed in the plots or provided as an error of the given values. The error $f$ of the measurements was calculated according to the standard deviation according to the multiplied measurement with Equation 6.4. The data was plotted as $\overline{\mathrm{x}} \pm f$ and the given error was rounded up if necessary.

$$
f=\sqrt{\frac{\sum(\mathrm{x}-\overline{\mathrm{x}})^{2}}{\mathrm{n}-1}}
$$

$\mathrm{n}=$ number of measurements

$\mathrm{x}=$ data point of the measurement

$\overline{\mathrm{x}}=$ mean value

\section{Tryptophan fluorescence and parallax measurements}

The J-1500 CD spectrometer was used for the probes of peptides solved in MeOH/TFE or incorporated in lipid membranes, which was prepared for the respective CD spectra. For this purpose, the EM detector was operated with a voltage of $400-800 \mathrm{~V}$. Therefore, the emission was adjusted at an intensity value of $\sim 0.7$. For the tryptophan fluorescence measurements a $1.5 \mathrm{~mL}$ quartz glass cuvette Suprasil ${ }^{\circledR}$ (QS) (HELLMA, Müllheim, Germany) was used. For the parallax measurements the FP-6200 was used. Table 6.5 contains the measurement parameters for the measurements performed at the J-1500 CD and the FP-6200. 
Table 6.5 Settings for the tryptophan measurements at the J-1500 CD and for the parallax measurements at the FP-6200.

\begin{tabular}{|c|c|}
\hline Meas. mode: & $\begin{array}{c}\text { Tryptophan } \\
\text { fluorescence }\end{array}$ \\
\hline D.I.T. & emission \\
\hline Em. bandwidth & $1.0 \mathrm{~s}$ \\
\hline Ex. bandwidth & $10.0 \mathrm{~nm}$ \\
\hline Ex. Wavelength & $2.0 \mathrm{~nm}$ \\
\hline Start/end & $290 \mathrm{~nm}$ \\
\hline Data pitch & $1.0 \mathrm{~nm}$ \\
\hline Accumulation & $2-5$ \\
\hline Sensitivity & $400-800 \mathrm{~V}$ \\
\hline & \\
\hline
\end{tabular}

\begin{tabular}{|cc|} 
& $\begin{array}{c}\text { Parallax } \\
\text { measurement }\end{array}$ \\
\hline Meas. mode: & Emission \\
\hline Response & Fast \\
\hline Em. bandwidth & $5.0 \mathrm{~nm}$ \\
\hline Ex. bandwidth & $5.0 \mathrm{~nm}$ \\
\hline Ex. Wavelength & $280 \mathrm{~nm}$ \\
\hline Start/end & $290-400 \mathrm{~nm}$ \\
\hline Data pitch & $1.0 \mathrm{~nm}$ \\
\hline Accumulation & 1 \\
\hline Sensitivity & Medium \\
\hline Scan speed & $60 \mathrm{~nm} / \mathrm{min}$ \\
\hline
\end{tabular}

For the tryptophan fluorescence and the parallax measurements, the peptides were solved in organic solvent $(300 \mu \mathrm{L}, \sim 50 \mu \mathrm{M})$ or undiluted vesicle suspension was prepared according to SOP 7.

\section{pH Fluorescence measurements and NBD distribution measurement}

For the $\mathrm{pH}$ dependent measurements, a FiveEasy ${ }^{\circledR} \mathrm{pH}$ meter from METTLER TOLEDO (Gießen, Germany) with a XS SENSOR (Carpi, Italy) Micro electrode and the FP-8500 were used. For the $\mathrm{pH}$ titration the device was calibrated with calibration solution from HANNA INSTRUMENTS (Woonsocket, USA) and a two-step calibration at a pH of 4 and 10 at $25{ }^{\circ} \mathrm{C}$. The device specific calibration slope was from $89 \%$ to $91 \%$. For the pH fluorescence measurements, the programme FixedWaveLentgh and for the determination of the NBD-labelled peptide Pep 15 distribution, the programme TimeCourse Measurement was used. Table 6.5 contains the measurement parameters for both measurements performed at the FP-8500. 
Table 6.6 Settings for $\mathrm{pH}$ fluorescence measurements at FP-8500.

\begin{tabular}{|c|c|}
\hline Response & $\begin{array}{c}\text { pH fluorescence } \\
\text { measurements }\end{array}$ \\
\hline Em. bandwidth & $1.0 \mathrm{~s}$ \\
\hline Ex. bandwidth & $5.0 \mathrm{~nm}$ \\
\hline Wavelength 1 & $5.0 \mathrm{~nm}$ \\
\hline Wavelength $\mathbf{2}$ & $490 / 520 \mathrm{~nm}$ \\
\hline Number of cycles & $55 / 520 \mathrm{~nm}$ \\
\hline Cycle interval & $30 \mathrm{~s}$ \\
\hline Sensitivity & $\mathrm{High}$ \\
\hline Temperature & $25^{\circ} \mathrm{C}$ \\
\hline
\end{tabular}

\begin{tabular}{|cc|}
\hline Response & $\begin{array}{c}\text { NBD distribution } \\
\text { measurements }\end{array}$ \\
\hline Em. bandwidth & $1.0 \mathrm{~s}$ \\
\hline Ex. bandwidth & $5.0 \mathrm{~nm}$ \\
\hline Ex. wavelength & $5.0 \mathrm{~nm}$ \\
\hline Ex. wavelength & $436 \mathrm{~nm}$ \\
\hline Data interval & $535 \mathrm{~nm}$ \\
\hline Cycle interval & $1 \mathrm{~s}$ \\
\hline Sensitivity & $30 \mathrm{~s}$ \\
\hline Temperature & medium \\
\hline Measurement range & $25^{\circ} \mathrm{C}$ \\
\hline & $0-1800 \mathrm{~s}$ \\
\hline
\end{tabular}

For the $\mathrm{pH}$ fluorescence measurements, a vesicle suspension was prepared according to Chapter SOP 7. Subsequently, $100 \mu \mathrm{L}$ of the vesicle suspension was diluted in $1900 \mu \mathrm{L}$ buffer $\left(\mathrm{PBS}=137 \mathrm{mM} \mathrm{NaCl}, 2.7 \mathrm{mM} \mathrm{KCl}, 10 \mathrm{mM} \mathrm{Na} 2 \mathrm{HPO}_{4}, 1.8 \mathrm{mM} \mathrm{KH}_{2} \mathrm{PO}_{4}, \mathrm{pH}\right.$ 7.4; HEPES $=10 \mathrm{mM} \mathrm{KCl}, 2 \mathrm{mM}$ HEPES) and $0.95 \mu \mathrm{L}$ of a $0.2 \mathrm{mM} \mathrm{CCCP}$ solution in DMSO was added. The titration was performed with $2-3 \mathrm{M} \mathrm{NaOH}$ and $\mathrm{HCl}$ starting from a $\mathrm{pH}$ of $\sim 7$ to a $\mathrm{pH}$ of 3 following a $\mathrm{pH}$ of $\sim 10$. For each $\mathrm{pH}$ value an individual measurement was performed following the conditions in Table 6.6. For the NBD distribution measurements a vesicle suspension was prepared according to Chapter SOP 7 and $40 \mu \mathrm{L}$ vesicle suspension was diluted in $1960 \mu \mathrm{L}$ buffer $\left(10 \mathrm{mM} \mathrm{Na}_{2} \mathrm{HPO}_{4}, 150 \mathrm{mM} \mathrm{NaCl}, \mathrm{pH}\right.$ 7.6). After the stabilisation of the fluorescence, $20 \mu \mathrm{L}$ of a freshly prepared $50 \mathrm{~mm}$ sodium dithionite solution in ultrapure water were added to the lipid solution. When no dramatic change in fluorescence was observed, a $10 \%$ solution of Triton X-100 in ultrapure water was added.

\section{$\mathrm{Ca}^{2+}$ fluorescence measurement}

The $\mathrm{Ca}^{2+}$ dependent measurements were performed at $20{ }^{\circ} \mathrm{C}$ by using a $\mathrm{Ca}^{2+}$ calibration buffer kit from INVITROGEN (Carlsbad, USA). This calibration buffer kit consists of two solutions, the Zero Free $\mathrm{Ca}^{2+}$ buffer $\left(10 \mathrm{mM} \mathrm{K} \mathrm{K}_{2}\right.$ EGTA, $100 \mathrm{mM} \mathrm{KCl}, 30 \mathrm{mM}$ MOPS, pH 7.2) and the $39 \mu \mathrm{M}$ Free $\mathrm{Ca}^{2+}$ buffer (10 mM CaEGTA, $100 \mathrm{mM} \mathrm{KCl,} 30 \mathrm{~mm}$ MOPS, $\mathrm{pH}$ 7.2). The combination of the two buffers in different ratio allows a free $\mathrm{Ca}^{2+}$ concentration from $0 \mu \mathrm{M}$ to $39 \mu \mathrm{M}$. In addition, different $\mathrm{Ca}^{2+}$ concentrations were prepared by dilution of a $1.0 \mathrm{M}$ or a $1.5 \mathrm{M} \mathrm{CaCl}_{2}$ solution in buffer $(100 \mathrm{mM} \mathrm{KCl}, 30 \mathrm{mM}$ MOPS, $\mathrm{pH}$ 7.2). The initial $\mathrm{CaCl}_{2} \times 6 \mathrm{H}_{2} \mathrm{O}$ mass was determined by weight. In Table 6.7 and Table 
6.8 the measurement parameters for the $\mathrm{Ca}^{2+}$ emission measurements and the $\mathrm{Ca}^{2+}$ titration measurements are given.

Table 6.7 Settings for $\mathrm{Ca}^{2+}$ emission measurements and $\mathrm{Ca}^{2+}$ titration measurements at the FP-8500.

\begin{tabular}{|cc|}
\hline Mode & $\begin{array}{c}\mathrm{Ca}^{2+} \text { emission } \\
\text { measurements }\end{array}$ \\
\hline Response & Excitation \\
\hline Em. bandwidth & $0.1 \mathrm{~s}$ \\
\hline Ex. bandwidth & $5.0 \mathrm{~nm}$ \\
\hline Start/end & $5.0 \mathrm{~nm}$ \\
\hline Em. wavelength & $544 \mathrm{~nm}$ \\
\hline Data interval & $0.5 \mathrm{~nm}$ \\
\hline Scan speed & $1000 \mathrm{~nm} / \mathrm{min}$ \\
\hline No. of accumulation & 3 \\
\hline Sensitivity & Medium \\
\hline Temperature & $20{ }^{\circ} \mathrm{C}$ \\
\hline Filter & Not used \\
\hline
\end{tabular}

\begin{tabular}{|cc|}
\hline Mode & $\begin{array}{c}\mathrm{Ca}^{2+} \text { titration } \\
\text { measurements }\end{array}$ \\
\hline Response & Emission \\
\hline Em. bandwidth & $0.1 \mathrm{~s}$ \\
\hline Ex. bandwidth & $5.0 \mathrm{~nm}$ \\
\hline Start/end & $5.0 \mathrm{~nm}$ \\
\hline Ex. wavelength & $510-700 \mathrm{~nm}$ \\
\hline Data interval & $0.5 \mathrm{~nm}$ \\
\hline Scan speed & $1000 \mathrm{~nm} / \mathrm{min}$ \\
\hline No. of accumulation & 3 \\
\hline Sensitivity & Medium \\
\hline Temperature & $20{ }^{\circ} \mathrm{C}$ \\
\hline Filter & Not used \\
\hline
\end{tabular}

For the $\mathrm{Ca}^{2+}$ titration measurements of Pep 18 and Pep 19 different excitation and emission wavelength were observed.

Table 6.8 Adjusted excitation wavelengths for the obtained peptides.

\begin{tabular}{|lcc|}
\hline & $\begin{array}{c}\text { Excitation } \\
\text { wavelengths }\end{array}$ & $\begin{array}{c}\text { Emission } \\
\text { wavelength }\end{array}$ \\
\hline Pep 18 & $416 / 496 \mathrm{~nm}$ & $544 \mathrm{~nm}$ \\
\hline Pep 19 & $405 / 488 \mathrm{~nm}$ & $539 \mathrm{~nm}$ \\
\hline
\end{tabular}

The $\mathrm{Ca}^{2+}$-sensor labelled peptides (Pep 18 and Pep 19) were dissolved in ultrapure water and equal volumes of the stock solution were distributed to the individual measurements ( $2 \mu \mathrm{M}$ Pep 18 and $\sim 15 \mu \mathrm{M}$ Pep 19). Several $\mathrm{Ca}^{2+}$ concentration dependent fluorescence dates were recorded per $\mathrm{Ca}^{2+}$ indicator at constant temperature, $\mathrm{pH}$ and indicator concentration.

For the $\mathrm{Ca}^{2+}$ emission and $\mathrm{Ca}^{2+}$ titration measurements of Pep 18, the following steps, based on the INVITROGEN protocol, were carried out:

1. A small amount of a Pep $\mathbf{1 8}$ stock solution in water was added to $1 \mathrm{~mL}$ Zero Free $\mathrm{Ca}^{2+}$ buffer (10 mM K $\mathrm{K}_{2}$ EGTA, $100 \mathrm{mM} \mathrm{KCl}, 30 \mathrm{mM}$ MOPS, pH 7.2) and four times the amount was added to $4 \mathrm{~mL}$ of $39 \mu \mathrm{M}$ Free $\mathrm{Ca}^{2+}$ buffer (10 mM CaEGTA, 100 mm KCl, 30 mm MOPS, pH 7.2). 
2. The fluorescence spectrum of Pep $\mathbf{1 8}$ in Zero Free $\mathrm{Ca}^{2+}$ buffer was recorded by using a total volume of $1 \mathrm{~mL}$.

3. In the following, the fluorescence intensity was measured at different $\mathrm{Ca}^{2+}$ concentrations. For this, the solution from the previous measurement was replaced by a certain volume of $39 \mu \mathrm{M}$ Free $\mathrm{Ca}^{2+}$ buffer containing the same concentration of $\mathrm{Ca}^{2+}$-sensor (see Table 6.9 a). The concentration of the sensor $(\sim 2 \mu \mathrm{M})$ and EGTA (10 mM) remained constant.

Additionally, two solutions were prepared with a $\mathrm{Ca}^{2+}$ concentration of $100 \mu \mathrm{M}$ and $2000 \mu \mathrm{M}$. Therefore, $\mathrm{CaCl}_{2}$ was dissolved in buffer (100 mM KCl, $30 \mathrm{~mm}$ MOPS, pH 7.2) to obtain a $1 \mathrm{mM}$ solution and this solution was diluted as required.

For the $\mathrm{Ca}^{2+}$ titration measurements of Pep 19, a $1 \mathrm{M} \mathrm{CaCl}_{2}$ solution in buffer $(100 \mathrm{mM}$ $\mathrm{KCl}, 30 \mathrm{~mm}$ MOPS, $\mathrm{pH}$ 7.2) was prepared and diluted to obtain the $\mathrm{Ca}^{2+}$ concentrations (seen in Table 6.9 b) given. Subsequently, in $1 \mathrm{~mL}$ of each $\mathrm{Ca}^{2+}$ containing solution was given a constant amount of Pep $19(\sim 15 \mu \mathrm{M})$.

Table 6.9 a) Serial dilution for the $\mathrm{Ca}^{2+}$ dependent fluorescence measurements of Pep 18 and b) $\mathrm{Ca}^{2+}$ concentration of aqueous buffer for $\mathrm{Ca}^{2+}$ dependent fluorescence measurements of Pep 19.

a)

\begin{tabular}{|ccc|}
\hline$[$ CaEGTA] & {$\left[\mathrm{Ca}^{2+}\right]_{\text {free }}$} & Replaced volume \\
\hline $0.0 \mathrm{mM}$ & $0 \mu \mathrm{M}$ & $900 \mu \mathrm{L}$ \\
\hline $9.0 \mathrm{mM}$ & $1.35 \mu \mathrm{M}$ & $100 \mu \mathrm{L}$ \\
\hline $9.1 \mathrm{mM}$ & $1.52 \mu \mathrm{M}$ & $111 \mu \mathrm{L}$ \\
\hline $9.2 \mathrm{mM}$ & $1.73 \mu \mathrm{M}$ & $125 \mu \mathrm{L}$ \\
\hline $9.3 \mathrm{mM}$ & $1.99 \mu \mathrm{M}$ & $143 \mu \mathrm{L}$ \\
\hline $9.4 \mathrm{mM}$ & $2.35 \mu \mathrm{M}$ & $167 \mu \mathrm{L}$ \\
\hline $9.5 \mathrm{mM}$ & $2.82 \mu \mathrm{M}$ & $200 \mu \mathrm{L}$ \\
\hline $9.6 \mathrm{mM}$ & $3.60 \mu \mathrm{M}$ & $250 \mu \mathrm{L}$ \\
\hline $9.7 \mathrm{mM}$ & $4.86 \mu \mathrm{M}$ & $333 \mu \mathrm{L}$ \\
\hline $9.8 \mathrm{mM}$ & $7.37 \mu \mathrm{M}$ & $500 \mu \mathrm{L}$ \\
\hline $9.9 \mathrm{mM}$ & $14.9 \mu \mathrm{M}$ & $500 \mu \mathrm{L}$ \\
\hline $9.95 \mathrm{mM}$ & $29.9 \mu \mathrm{M}$ & - \\
\hline $10.0 \mathrm{mM}$ & $39.0 \mu \mathrm{M}$ & - \\
\hline- & $100 \mu \mathrm{M}$ & - \\
\hline- & $2000 \mu \mathrm{M}$ & - \\
\hline
\end{tabular}

b)

\begin{tabular}{|c|}
\hline$\left[\mathrm{Ca}^{2+}\right]_{\text {free }}$ \\
\hline $0 \mu \mathrm{M}$ \\
\hline $15.6 \mu \mathrm{M}$ \\
\hline $31.3 \mu \mathrm{M}$ \\
\hline $39.0 \mu \mathrm{M}$ \\
\hline $62.5 \mu \mathrm{M}$ \\
\hline $125 \mu \mathrm{M}$ \\
\hline $250 \mu \mathrm{M}$ \\
\hline $500 \mu \mathrm{M}$ \\
\hline $1.0 \mathrm{mM}$ \\
\hline $15.6 \mathrm{mM}$ \\
\hline $31.3 \mathrm{mM}$ \\
\hline $62.5 \mathrm{mM}$ \\
\hline $125 \mathrm{mM}$ \\
\hline $250 \mathrm{mM}$ \\
\hline $500 \mathrm{mM}$ \\
\hline $1.0 \mathrm{M}$ \\
\hline
\end{tabular}

The $K_{\mathrm{d}}$ of Pep 18 was obtained by a double-logarithmic application of the $\mathrm{Ca}^{2+}$ concentration $\log \left(\left[\mathrm{Ca}^{2+}\right]\right)$ against $\log \left\{\left(\mathrm{R}-\mathrm{R}_{\min }\right) /\left(\mathrm{R}_{\max }-\mathrm{R}\right)\right\}$. $\mathrm{R}$ is the ratio of the fluorescence emission by two different excitation wavelengths at a certain $\mathrm{Ca}^{2+}$ concentration. $\mathrm{R}_{\min }$ is the 
ratio at $0 \mu \mathrm{M}$ free $\mathrm{Ca}^{2+}$ and $\mathrm{R}_{\max }$ is the ratio for a fully saturated sensor. The intersection of the straight line at $\mathrm{y}=0$ corresponds to the logarithmic $K_{\mathrm{d}}$ value.

For the study of fluorescence dependence versus other divalent metal ions, the salts $\mathrm{ZnCl}_{2}$, $\mathrm{MgCl}_{2} \times 6 \mathrm{H}_{2} \mathrm{O}$ and $\mathrm{CaCl}_{2} \times 2 \mathrm{H}_{2} \mathrm{O}$ were used. The salts were dissolved in an EGTA-free buffer (100 mM KCl, $30 \mathrm{mM}$ MOPS, $\mathrm{pH} 7.2)$ to a concentration of $0.5 \mathrm{M}$. Subsequently, a certain amount of salt solution was added to a solution of $\mathrm{Ca}^{2+}$-sensor in buffer $(100 \mathrm{mM}$ $\mathrm{KCl}, 30 \mathrm{~mm}$ MOPS, $\mathrm{pH} 7.2)$ to obtain a final concentration of $100 \mu \mathrm{M}$ or $2000 \mu \mathrm{M}$. The sensor concentration was kept constant in all measurements.

The salts $\mathrm{NiCl} \times 6 \mathrm{H}_{2} \mathrm{O}, \mathrm{MnCl} \times 4 \mathrm{H}_{2} \mathrm{O}$ and $\mathrm{CoCl}$ were used to measure the fluorescence quenching by ions. Therefore, the salts were dissolved in an EGTA-free buffer (100 mM $\mathrm{KCl}, 30 \mathrm{mM}$ MOPS, $\mathrm{pH} 7.2$ ) to a concentration of $0.5 \mathrm{M}$. Subsequently, a certain amount of salt solution was added to a solution of $\mathrm{Ca}^{2+}$-sensor in buffer $(100 \mathrm{mM} \mathrm{KCl}, 30 \mathrm{mM}$ MOPS, $\mathrm{pH}$ 7.2) to obtain the given concentration. The sensor concentration was kept constant in all measurements.

\subsection{Standard operating procedures (SOPs)}

SOP 1 General synthesis of $N$-protected $D$ - $\beta^{3}$-amino acids<smiles>[R]C(CC(=O)O)N[R6]#CC</smiles>

Scheme 6.1 General reaction scheme for $\beta^{3}$-amino acids starting from $\alpha$-amino acids $(P G=$ protection group).

The respective $N$-protected $\alpha$-amino acid (1.00 eq) was dissolved in dry THF $(4.7 \mathrm{~mL} / \mathrm{mmol})$ and cooled down to $0{ }^{\circ} \mathrm{C}$. Isobutyl chloroformate $(1.10 \mathrm{eq})$ and triethylamine $(1.10 \mathrm{eq})$ were added at $0{ }^{\circ} \mathrm{C}$, followed by stirring at $0{ }^{\circ} \mathrm{C}$ for $30 \mathrm{~min}$. Under light exclusion diazomethane solution in diethyl ether $(0.6 \mathrm{M}, 2.00 \mathrm{eq})$ was added to the reaction mixture and stirred at RT for $5 \mathrm{~h}$. The reaction was quenched with acetic acid $(0.1 \mathrm{~mL} / \mathrm{mmol})$ followed by the addition of an aqueous solution of sodium bicarbonate $(6 \%, 9.4 \mathrm{~mL} / \mathrm{mmol})$. The aqueous phase was extracted with ethyl acetate $(3 \times 100 \mathrm{~mL})$. The combined organic phases were washed with saturated aqueous solution of ammonium chloride $(3 \times 60 \mathrm{~mL})$ and saturated aqueous solution of sodium chloride $(60 \mathrm{~mL})$, followed by drying over magnesium sulphate. The solvent was removed under reduced pressure and the crude product was received as a yellow oil or solid. When the crude product was brown or red, side products were removed by flash column chromatography (Pentane/EtOAc 1:1). 
For the following WOLFF rearrangement two reaction conditions could be used:

1. The diazo ketone (1.00 eq) of the protected amino acid was dissolved in $\mathrm{THF} / \mathrm{H} 2 \mathrm{O}$ (9:1) and silver benzoate ( 0.10 eq) was added. The reaction mixture was sonicated at RT for $2 \mathrm{~h}$ and a small amount of water was added.

2. The diazo ketone $(1.00 \mathrm{eq})$ of the protected amino acid was dissolved in dioxane/ $\mathrm{H}_{2} \mathrm{O}(2: 1)$ and silver benzoate $(0.10 \mathrm{eq})$ was added. The reaction mixture was placed in a domestic microwave $(460 \mathrm{~W}, 1 \mathrm{~min})$ and the organic solvent was removed under reduced pressure.

Afterwards, the respective WOLFF rearrangement mixture was adjusted to a $\mathrm{pH} 2-3$ by adding $2 \mathrm{M}$ hydrochloric acid. Then, the aqueous phase was extracted with ethyl acetate $(3 \times 100 \mathrm{~mL})$. The combined organic phases were dried over magnesium sulphate. The solvent was removed under reduced pressure and the obtained crude product was dissolved in DCM and precipitate in cold pentane to get the product which was manageable and pure enough for the peptide synthesis (impurities were still contained). For a pure product the crude product was purified by flash column chromatography (Pentane/EE or $\mathrm{DCM} / \mathrm{MeOH})$.

\section{SOP 2 Manual loading of the resin with the first amino acid}

The loading of the rink amide MBHA resin (1.00 eq) was performed in a BECTONDICKINSON (BD; Heidelberg, Germany) discardit II syringe equipped with a PE-frit. The resin was swollen in DMF for $2 \mathrm{~h}$. The resin-bound Fmoc protection group was cleaved two times using a solution of NMP/piperidine/DBU $(v / v / v ; 79: 20: 1)$ under microwave irradiation: (a) $30 \mathrm{~s}, 25 \mathrm{~W}, 50{ }^{\circ} \mathrm{C}$ (b) $3 \mathrm{~min}, 35 \mathrm{~W}, 65^{\circ} \mathrm{C}$. The resin was washed with DMF $(3 \times)$, DCM $(3 \times)$ and DMF $(3 \times)$. The amino acid building block (5.00 eq) and HOBt (5.00 eq) were dissolved in DMF ( $2 \mathrm{~mL} / \mathrm{mmol})$ and DIC (5.00 eq) was added. The coupling solution was given to the resin and coupled microwave-assisted $\left(35 \mathrm{~W}, 65^{\circ} \mathrm{C}\right.$, $15 \mathrm{~min})$. The resin was washed with $\operatorname{DMF}(3 \times), \mathrm{MeOH}(3 \times)$ and $\mathrm{DCM}(3 \times)$ and dried under reduced pressure. The resign loading was checked by SOP3 and has to be between $0.3 \mathrm{mmol} / \mathrm{g}$ and $0.4 \mathrm{mmol} / \mathrm{g}$. After a successful resign loading free amino groups were capped with acetic anhydride/NMP $\left(v / v ; 20: 80,3 \mathrm{~min}, 35 \mathrm{~W}, 65^{\circ} \mathrm{C}\right)$. Afterwards the resign was washed again with $\mathrm{DMF}(3 \times), \mathrm{MeOH}(3 \times)$ and $\mathrm{DCM}(3 \times)$ and dried under reduced pressure. 
The NovaPeg rink amide resin LL $(0.19 \mathrm{mmol} / \mathrm{g})$ was loaded with the first amino acids followed by the normal coupling protocol for the respective peptide.

\section{SOP 3 KAISER test}

The KAISER test was performed for checking the presence of free amino groups during the SPPS. A small amount of dry or $\mathrm{MeOH}$-wet resin was placed in a glass tube. $2-3$ drops of each solution were added to the tube and heated to $100{ }^{\circ} \mathrm{C}$ for $2-5 \mathrm{~min}$. A negative test was performed when the solution and resin were still yellow, which indicated none free amino groups. A positive test was performed when the resin or the solution turned to blue, which showed the presence of free amino group. The following solutions were prepared for the KAISER test:

Reagent 1: $1.0 \mathrm{~g}$ ninhydrin in $20 \mathrm{~mL}$ ethanol,

Reagent 2: $16 \mathrm{mg}$ phenol in $4 \mathrm{~mL}$ ethanol,

Reagent 3: $\quad 0.50 \mathrm{~mL}$ solution of $\mathrm{KCN}(1 \mathrm{mM})$ diluted to $25 \mathrm{~mL}$ with pyridine.

\section{SOP 4 Determination of resin loading ${ }^{[11]}$}

The determination of resin loading of the rink amide MBHA resin was performed by SOP 2. A small amount of resin $(5-10 \mathrm{~g})$ was given in a $10 \mathrm{~mL}$ volumetric flask. $40 \mu \mathrm{L} 1,8$ diazabicyclo[5.4.0]undec-7-en (DBU) and $2 \mathrm{~mL}$ DMF were added and the solution was mixed mechanical or sonicated at RT for $2 \mathrm{~h}$. This solution was filled up to $10 \mathrm{~mL}, 2 \mathrm{~mL}$ of these were given to a $25 \mathrm{~mL}$ volumetric flask and diluted to $25 \mathrm{~mL}$ with ACN. The absorption measurement was performed at $304 \mathrm{~nm}$ and corrected by a blank probe without resin. The resin loading was calculated by following Equation 6.5.

$$
\left[\frac{\mathrm{mmol}}{\mathrm{g}}\right]=\frac{\mathrm{A}_{304} \cdot 16.4}{m_{\mathrm{resin}}}
$$

$\mathrm{A}_{304}=$ absorption at $304 \mathrm{~nm}$ corrected by blank probe

$\mathrm{m}_{\text {resin }}=$ mass of resin $[\mathrm{mg}]$

\section{SOP 5 Manual microwave-supported SPPS}

The synthesis of the $\beta$-peptides was performed with manual microwave-assisted solid phase peptide synthesis (SPPS) in a BD-syringe equipped with a PE-filter. All peptides were synthesised on a NovaPeg resin $(0.18 \mathrm{mmol} / \mathrm{g})$, with the exception of Pep 1 - Pep 4 and Pep 9-Pep 10, which were synthesised on a rink amide MBHA resin (0.3$0.5 \mathrm{mmol} / \mathrm{g}$ ). The first amino acid on the rink amide MBHA resin was loaded according to 
SOP 2. The resin was swelled in DMF for at least $1 \mathrm{~h}$ and it was washed between the reaction steps for at least 3 times with NMP, DMF, DCM or a mixture of DMF/MeOH $(1: 1)$.

1. The resin-bound Fmoc-group was deprotected twice by using a solution of NMP/piperidine/DBU $(v / v / v ; 79: 20: 1)$ under microwave irradiation. The two deprotection steps differ in the reaction time and temperature $\left(1.30 \mathrm{~s}, 25 \mathrm{~W}, 50{ }^{\circ} \mathrm{C}\right.$; 2. $\left.3 \mathrm{~min}, 35 \mathrm{~W}, 65^{\circ} \mathrm{C}\right)$.

2. After washing, the respective amino acid (5.00 eq) was dissolved in a solution of 0.5 M HOAt/HATU (5.00 eq/4.90 eq) in DMF and 2.0 M DIPEA (10.00 eq) in NMP. The activated amino acid was added to the resin and microwave-assisted coupling ( $15 \mathrm{~min}, 35 \mathrm{~W}, 65^{\circ} \mathrm{C}$ ) was performed. This coupling step was performed for the hydrophobic amino acids once (Pep 1-Pep 4, Pep 9, Pep 10, Pep 12), twice (Pep 11, Pep 15) or thrice (Pep 5-Pep 8). D- $\beta^{3}$-hTrp and ACHC was always coupled twice.

3. Free amino groups were capped with acetic anhydride/NMP ( $v / v ; 20: 80)$ microwave-supported ( $\left.3 \mathrm{~min}, 35 \mathrm{~W}, 65^{\circ} \mathrm{C}\right)$.

After completion of the $\beta$-peptide sequence, the final wash was performed at the MBHA resin with DCM and at the NovaPeg resin with $\mathrm{MeOH}$ followed by diethyl ether. Finally, the resin was dried under reduced pressure. Cleavage from the resin and removal of the protecting groups was done with TFA/ $\mathrm{H}_{2} \mathrm{O} / \mathrm{TIS}(v / v / v ; 95: 2.5: 2.5)$ for $2 \mathrm{~h}$. The solvent was removed in a nitrogen stream and the final crude $\beta$-peptides were precipitated with diethyl ether and purified by using HPLC.

\section{SOP 6 Automatic microwave-supported SPPS}

The automatic microwave-supported SPPS was performed on a peptide synthesizer equipped with a DISCOVER microwave reaction cavity (CEM, Kamp-Lintfort, Germany). The resin was not preswelled and two coupling protocols were established. The exact reaction cycles for the performed synthesis scales as well as the used coupling solutions are listed in Appendix A. Method 1 was used for Pep Met 1 and Method 2 was used for Pep Met 2, Pep 13, Pep 14, Pep 16, Pep 18 - Pep 20. 


\section{SOP 7 Preparation of large unilamellar vesicles (LUV)}

\section{General}

Large unilamellar vesicles (LUV) were prepared by extradition a rehydrated lipid film. The lipid film was produced by taking a certain amount of lipid dissolved in chloroform and by adding peptide dissolved in $\mathrm{MeOH}$ or TFE. The solvent was dispersed at $50{ }^{\circ} \mathrm{C}$ with a nitrogen stream and the residual solvent was removed in vacuum at $40{ }^{\circ} \mathrm{C}$ overnight. Subsequently, the lipid film was rehydrated with ultrapure water or buffer and incubated at $50{ }^{\circ} \mathrm{C}$ for at least $1 \mathrm{~h}$. The suspension was mixed mechanically $(4 \times 1 \mathrm{~min}$, after each mixing step the sample was left for $5 \mathrm{~min}$ at $50^{\circ} \mathrm{C}$ ). Afterwards, the suspension was extruded 31 times through a polycarbonate membrane with a defined pore size in a LiposoFast mini-extruder.

\section{CD and Tryptophan fluorescence measurements}

For the $\mathrm{CD}$ and tryptophan fluorescence measurements, $0.30 \mathrm{mg}$ of the respective lipid and $5 \mathrm{~mol} \%$ or $6 \mathrm{~mol} \%$ of the $\beta$-peptide dissolved in TFE or MeOH were mixed and dried (P/L ratio $5: 100$ or $6: 100$ ). The rehydration was performed with $500 \mu \mathrm{L}$ ultrapure water and the suspension was extruded through a membrane with a pore size of $100 \mathrm{~nm}$.

\section{Parallax measurement}

For the parallax measurements, $0.3 \mu \mathrm{mol}$ of the respective lipid (varying amount of DOXYL-labelled lipid $0 \%-13 \%$ ) and $0.5 \mathrm{~mol} \%$ of the $\beta$-peptide dissolved in TFE or $\mathrm{MeOH}$ were mixed and dried $(\mathrm{P} / \mathrm{L}$ ratio 1:200). The rehydration was performed with $500 \mu \mathrm{L}$ ultrapure water and the suspension was extruded through a membrane with a pore size of $100 \mathrm{~nm}$.

\section{pH Fluorescence measurements and NBD distribution measurement}

For the $\mathrm{pH}$ fluorescence measurements, a total lipid amount of substance of $0.38 \mu \mathrm{mol}$ and $0.5 \mathrm{~mol} \%$ of the $\beta$-peptide dissolved in TFE or $\mathrm{MeOH}$ were mixed and dried (P/L ratio 1:200). The rehydration was performed with $500 \mu \mathrm{L}$ buffer and the suspension was extruded through a membrane with a pore size of $100 \mathrm{~nm}$ or $400 \mathrm{~nm}$.

\section{SOP 8 Functionalisation of $\mathrm{Ca}^{2+}$-sensors}

The protected $\mathrm{Ca}^{2+}$-sensor $(1.00 \mathrm{eq})$ and the respective hydrochlorid salt $(1.00 \mathrm{eq})$ were added in a flame dried flask. Dry ethanol $(14.6 \mathrm{~mL} / \mathrm{mmol})$ was added under argon atmosphere. The reaction was heated under reflux for $5 \mathrm{~h}^{[37]}$ As required the product was purified by flash chromatography. The hydrochlorid salts of the commercial available 2,2'- 
dithiobis(ethylamine) and $\beta$-alanine as well as the hydrochlorid salts of the synthesised (analysis data not given) $N$-(2-aminoethyl)maleimide ${ }^{[304,305]}$ and $(S)$-2-pyridylthio cysteamine ${ }^{[306]}$ were obtained by lyophilisation with diluted $\mathrm{HCl}$ solution.

\section{SOP 9 Labelling of $\boldsymbol{\beta}$-peptides with carboxyfluorescein as $\mathbf{p H}$-sensor}

For the labelling of carboxyfluorescein to the resin-bound peptide three microwave-supported conditions were developed. Therefore, the fluorophore and the solid coupling reagents were diluted in a minimum of DMF and the liquid coupling reagents were added in small portion. Subsequently, the coupling mixture was added to the dry resin.

1. DIC (10.00 eq)/Oxyma (5.00 eq)/carboxyfluorescein (5.00 eq) double coupling $\left(25 \mathrm{~W}, 5 \mathrm{~min}, 90^{\circ} \mathrm{C}\right)$,

2. HATU (4.90 eq)/HOAt (5.00 eq)/DIPEA (10.00 eq)/carboxyfluorescein (5.00 eq), double coupling $\left(25 \mathrm{~W}, 15 \mathrm{~min}, 75^{\circ} \mathrm{C}\right)$,

3. PyAOP (5.00 eq)/HOAt (5.00 eq)/DIPEA (10.00 eq)/carboxyfluorescein (5.00 eq), double coupling $\left(25 \mathrm{~W}, 60 \mathrm{~min}, 75^{\circ} \mathrm{C}\right)$.

After the successful labelling the washed resin was treated with a solution of $\mathrm{NMP} /$ piperidine $(v / v ; 80: 20)$ at $\mathrm{RT}$ for $15 \mathrm{~min}$ to remove the phenolic ester. ${ }^{\text {[230] }}$

\section{SOP 10 Labelling of $\beta$-peptides with $\mathrm{Ca}^{2+}$-sensors 38 and 46 $\mathrm{Ca}^{2+}$-sensor 38}

The $\mathrm{Ca}^{2+}$-sensor 38 (3.00 eq), PyAOP (6.00 eq) and HOAt (3.00 eq) were dissolved in DMF (15 mL/mmol based on resin-bound peptide) and DIPEA (6.00 eq) was added subsequently in three fractions. This reaction mixture was added to the resin and if required DMF was added until the resin was completely covered. The fluorophore was coupled microwave-supported to the resin-bound peptide $\left(20 \mathrm{~W}, 2 \mathrm{~h}, 65^{\circ} \mathrm{C}\right.$ (up to $10{ }^{\circ} \mathrm{C}$ overshoot)) for $2 \mathrm{~h}$.

$\mathrm{Ca}^{2+}$-sensor 46

The $\mathrm{Ca}^{2+}$-sensor (5.00 eq), PyAOP (10.00 eq) and HOAt (5.00 eq) were dissolved in DMF (15 mL/mmol based on resin-bound peptide) and subsequently DIPEA (15.00 eq) was added in three fractions. This reaction mixture was added to the resin and if required DMF was added until the resin was completely covered. The fluorophore was coupled microwave-supported to the resin-bound peptide $\left(20 \mathrm{~W}, 2 \mathrm{~h}, 65^{\circ} \mathrm{C}\right.$ (up to $10{ }^{\circ} \mathrm{C}$ overshoot)) for $2 \mathrm{~h}$. 


\subsection{Synthesis}

\subsection{1 $\beta^{3}$-Homoamino acids and coupling reagent DMT/NMM/TOS}

(R)-7-tert-Butoxycarbonylamino-3-(9-fluorenylmethyloxycarbonyl)-aminoheptanoic acid (Fmoc-D- $\beta^{3}$-hLys $($ Boc $\left.)-O H\right)$

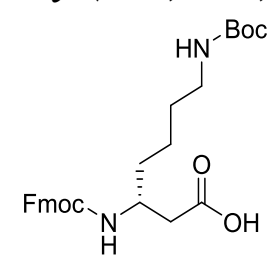

47

$\mathrm{C}_{27} \mathrm{H}_{34} \mathrm{~N}_{2} \mathrm{O}_{6}$

$482.24 \mathrm{~g} / \mathrm{mol}$

Following SOP 1 the synthesis was achieved starting with Fmoc-D-Lys(Boc)-OH (5.00 g, $10.10 \mathrm{mmol}, 1.00 \mathrm{eq})$ to yield the product $47(4.57 \mathrm{~g}, 9.48 \mathrm{mmol}, 89 \%)$ as a yellow solid.

${ }^{\mathbf{1}} \mathbf{H}-\mathbf{N M R}\left(300 \mathrm{MHz},\left[\mathrm{D}_{6}\right] \mathrm{DMSO}\right): \delta(\mathrm{ppm})=1.11-1.45\left(\mathrm{~m}, 15 \mathrm{H}, \mathrm{C}_{3}, \mathrm{C}_{2}\right), 2.24-2.43$ (m, 2H, $\left.\underline{\mathrm{C}}_{2}\right), 2.81-2.94\left(\mathrm{~m}, 2 \mathrm{H}, \mathrm{C}_{2}\right), 3.67-3.83(\mathrm{~m}, 1 \mathrm{H}, \beta-\mathrm{C} \underline{\mathrm{H}}), 4.17-4.33$ (m, 3H,

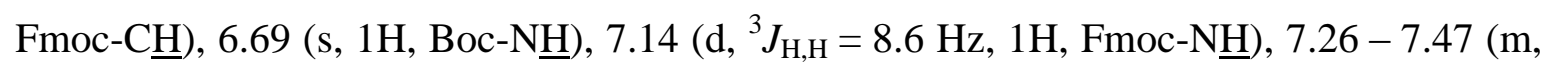
$4 \mathrm{H}$, Fmoc-C프), $7.68\left(\mathrm{~d},{ }^{3} J_{\mathrm{H}, \mathrm{H}}=7.7 \mathrm{~Hz}, 2 \mathrm{H}\right.$, Fmoc-C $\left.\underline{\mathrm{H}}\right), 7.88\left(\mathrm{~d},{ }^{3} J_{\mathrm{H}, \mathrm{H}}=7.7 \mathrm{~Hz}, 2 \mathrm{H}\right.$, Fmoc$\mathrm{C} \underline{\mathrm{H}}), 12.11\left(\mathrm{~s}_{\mathrm{br}}, 1 \mathrm{H}, \mathrm{COO} \underline{\mathrm{H}}\right)$.

MS (ESI, $\mathrm{MeOH}): \mathrm{m} / \mathrm{z}=505.2[\mathrm{M}+\mathrm{Na}]^{+} ; 987.5[2 \mathrm{M}+\mathrm{Na}]^{+}$.

HR-MS (ESI, MeOH):

$$
\mathrm{C}_{27} \mathrm{H}_{34} \mathrm{~N}_{2} \mathrm{O}_{6} \mathrm{Na}[\mathrm{M}+\mathrm{Na}]^{+} \quad \text { calc.: 505.2309 mesur.: 505.2311. }
$$


(R)-3-(9-Fluorenylmethyloxycarbonyl)-amino-5-methyl-hexanoic acid (Fmoc$\left.D-\beta^{3}-h L e u-O H\right)$

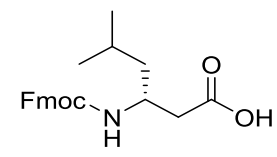

48

$\mathrm{C}_{22} \mathrm{H}_{25} \mathrm{NO}_{4}$

$367.18 \mathrm{~g} / \mathrm{mol}$

Following SOP 1 the synthesis was achieved starting with Fmoc-D-Leu-OH (10.00 g, $28.30 \mathrm{mmol}, 1.00 \mathrm{eq})$ to yield the product $48(8.50 \mathrm{~g}, 23.10 \mathrm{mmol}, 82 \%)$ as a colourless or yellow solid.

${ }^{1}$ H-NMR $\left(300 \mathrm{MHz},\left[\mathrm{D}_{6}\right] \mathrm{DMSO}\right): \delta(\mathrm{ppm})=0.86\left(\mathrm{~d},{ }^{3} \mathrm{~J}_{\mathrm{H}, \mathrm{H}}=6.5 \mathrm{~Hz}, 6 \mathrm{H}, \mathrm{CH}_{3}\right), 1.11-$ 1.27 (m, 1H, $\left.\underline{\mathrm{C}}_{2}\right), 1.32-1.51\left(\mathrm{~m}, 1 \mathrm{H}, \underline{\mathrm{C}}_{2}\right), 1.49-1.62(\mathrm{~m}, 1 \mathrm{H}, \mathrm{C} \underline{\mathrm{H}}), 2.23-2.45$ (m,

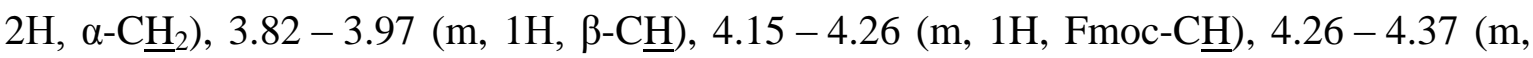
$2 \mathrm{H}$, Fmoc-C $\left.\underline{\mathrm{H}}_{2}\right), 7.08-7.17\left(\mathrm{~d},{ }^{3} J_{\mathrm{H}, \mathrm{H}}=8.8 \mathrm{~Hz}, 1 \mathrm{H}\right.$, Fmoc-NH$), 7.28-7.36$ (m, 2H, Fmoc-

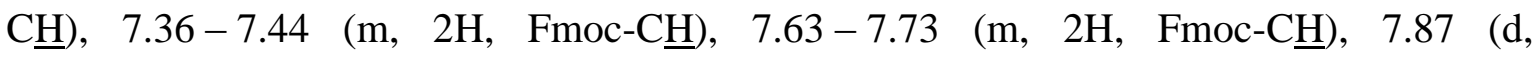
$\left.{ }^{3} J_{\mathrm{H}, \mathrm{H}}=7.9 \mathrm{~Hz}, 2 \mathrm{H}, \mathrm{Fmoc}-\mathrm{C} \underline{\mathrm{H}}\right), 12.12\left(\mathrm{~s}_{\mathrm{br}}, 1 \mathrm{H}, \mathrm{COO} \underline{\mathrm{H}}\right)$.

MS (ESI, MeOH): m/z = 390.2 [M+ Na $]^{+} ; 757.4[2 \mathrm{M}+\mathrm{Na}]^{+}$.

HR-MS (ESI, MeOH):

$\mathrm{C}_{22} \mathrm{H}_{25} \mathrm{NO}_{4} \mathrm{Na}[\mathrm{M}+\mathrm{Na}]^{+} \quad$ calc.: $390.1676 \quad$ mesur.: 390.1676 .

(R)-3-(9-Fluorenylmethyloxycarbonyl)-amino-4-methyl-pentanoic acid (Fmoc$D-\beta^{3}-h$ Val-OH)

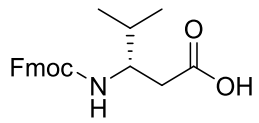

49

$\mathrm{C}_{21} \mathrm{H}_{23} \mathrm{NO}_{4}$

$353.16 \mathrm{~g} / \mathrm{mol}$

Following SOP 1 the synthesis was achieved starting with Fmoc-D-Val-OH (10.00 g, $29.50 \mathrm{mmol}, 1.00 \mathrm{eq})$ to yield the product $49(8.65 \mathrm{~g}, 24.50 \mathrm{mmol}, 83 \%)$ as a colourless or yellow solid. 
${ }^{1}$ H-NMR (300 MHz, [D 6 ]DMSO): $\delta(\mathrm{ppm})=0.67-0.94\left(\mathrm{~m}, 6 \mathrm{H}, \underline{\mathrm{C}}_{3}\right), 1.65-1.82(\mathrm{~m}$, $\left.1 \mathrm{H}, \mathrm{C}_{2}\right), 2.24-2.48\left(\mathrm{~m}, 2 \mathrm{H}, \alpha-\underline{\mathrm{H}}_{2}\right), 3.69-3.84(\mathrm{~m}, 1 \mathrm{H}, \beta-\mathrm{C} \underline{\mathrm{H}}), 4.16-4.39$ (m, 3H, Fmoc-C므, Fmoc-C $\left.\underline{\mathrm{H}}_{2}\right), 7.20\left(\mathrm{~d},{ }^{3} J_{\mathrm{H}, \mathrm{H}}=9.0 \mathrm{~Hz}, 1 \mathrm{H}, \mathrm{Fmoc}-\mathrm{N} \underline{\mathrm{H}}\right), 7.25-7.47$ (m, 4H, Fmoc-

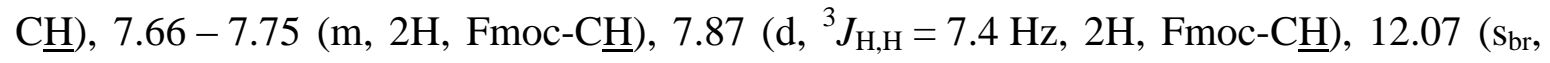
$1 \mathrm{H}, \mathrm{COO} \underline{\mathrm{H}})$.

MS (ESI, MeOH): m/z = $376.2[\mathrm{M}+\mathrm{Na}]^{+} ; 729.3[2 \mathrm{M}+\mathrm{Na}]^{+} ; 1082.5[3 \mathrm{M}+\mathrm{Na}]^{+}$.

HR-MS (ESI, MeOH):

$\begin{array}{lll}\mathrm{C}_{21} \mathrm{H}_{24} \mathrm{NO}_{4}[\mathrm{M}+\mathrm{H}]^{+} & \text {calc.: } 354.1700 & \text { mesur.: 354.1700; } \\ \mathrm{C}_{21} \mathrm{H}_{23} \mathrm{NO}_{4} \mathrm{Na}[\mathrm{M}+\mathrm{Na}]^{+} & \text {calc.: } 376.1519 & \text { mesur.: } 376.1519 .\end{array}$

(R)-3-(9-Fluorenylmethyloxycarbonyl)-amino-4-methyl-hexanoic acid (Fmoc$D-\beta^{3}$-hIle-OH)

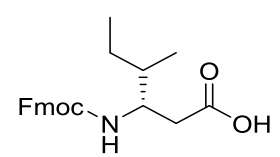

50

$\mathrm{C}_{22} \mathrm{H}_{25} \mathrm{~N}_{3} \mathrm{O}_{4}$

$367.18 \mathrm{~g} / \mathrm{mol}$

Following SOP 1 the synthesis was achieved starting with Fmoc-D-Ile-OH $(5.00 \mathrm{~g}$, $14.20 \mathrm{mmol}, 1.00 \mathrm{eq})$ to yield the product $\mathbf{5 0}(4.00 \mathrm{~g}, 10.90 \mathrm{mmol}, 77 \%)$ as a colourless or yellow solid.

${ }^{\mathbf{1}} \mathbf{H}-\mathbf{N M R}\left(300 \mathrm{MHz},\left[\mathrm{D}_{6}\right] \mathrm{DMSO}\right): \delta(\mathrm{ppm})=0.63-0.90\left(\mathrm{~m}, 6 \mathrm{H}, \mathrm{C}_{3}\right), 0.96-1.18(\mathrm{~m}$, $\left.1 \mathrm{H}, \mathrm{C}_{2}\right), 1.29-1.60\left(\mathrm{~m}, 2 \mathrm{H}, \underline{\mathrm{C}}_{2}, \mathrm{C} \underline{\mathrm{H}}\right) 2.21-2.45\left(\mathrm{~m}, 2 \mathrm{H}, \alpha-\underline{\mathrm{C}}_{2}\right), 3.73-3.89(\mathrm{~m}, 1 \mathrm{H}$, $\beta-\mathrm{CH}), 4.15-4.40$ (m, 3H, Fmoc-C태, Fmoc-C $\left.\underline{H}_{2}\right), 7.22\left(\mathrm{~d},{ }^{3} J_{\mathrm{H}, \mathrm{H}}=8.9 \mathrm{~Hz}, 2 \mathrm{H}\right.$, Fmoc-

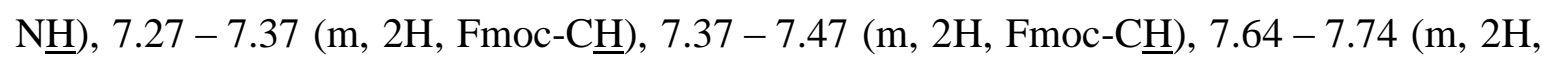
Fmoc-C마), $7.87\left(\mathrm{~d},{ }^{3} J_{\mathrm{H}, \mathrm{H}}=7.4 \mathrm{~Hz}, 2 \mathrm{H}, \mathrm{Fmoc}-\mathrm{CH}\right), 11.86\left(\mathrm{~s}_{\mathrm{br}}, 1 \mathrm{H}, \mathrm{COO} \underline{\mathrm{H}}\right)$.

MS (ESI, MeOH): m/z = 368.2[M + H] $]^{+} 390.2[\mathrm{M}+\mathrm{Na}]^{+} ; 757.4[2 \mathrm{M}+\mathrm{Na}]^{+}$.

HR-MS (ESI, MeOH):

$$
\begin{array}{lll}
\mathrm{C}_{22} \mathrm{H}_{26} \mathrm{~N}_{3} \mathrm{O}_{4}[\mathrm{M}+\mathrm{H}]^{+} & \text {calc.: } 368.1856 & \text { mesur.: 368.1850; } \\
\mathrm{C}_{22} \mathrm{H}_{25} \mathrm{~N}_{3} \mathrm{O}_{4} \mathrm{Na}[\mathrm{M}+\mathrm{Na}]^{+} & \text {calc.: } 390.1676 & \text { mesur.: 390.1676. }
\end{array}
$$


(R)-4-(1'-tert-Butoxycarbonyl-indo-3 '-yl)-3-(9-fluorenylmethyloxycarbonyl)amino-butanoic acid (Fmoc-D- $\left.\beta^{3}-h T r p(B o c)-O H\right)$

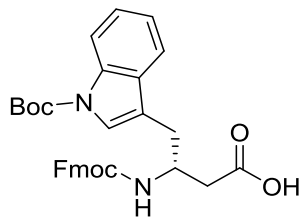

51

$\mathrm{C}_{32} \mathrm{H}_{32} \mathrm{~N}_{2} \mathrm{O}_{6}$

$540.23 \mathrm{~g} / \mathrm{mol}$

Following SOP 1 the synthesis was achieved starting with Fmoc-D-Trp(Boc)-OH (10.00 g, $19.00 \mathrm{mmol}, 1.00 \mathrm{eq})$ to yield the product $51(8.09 \mathrm{~g}, 15.00 \mathrm{mmol}, 79 \%)$ as a yellow solid.

${ }^{1}$ H-NMR (300 MHz, [D 6 ]DMSO): $\delta(\mathrm{ppm})=1.55\left(\mathrm{~s}, 9 \mathrm{H}, \mathrm{CH}_{3}\right), 2.45-2.57\left(\mathrm{~m}, 2 \mathrm{H}, \mathrm{C}_{2}\right.$, overlaid by solvent), $2.88\left(\mathrm{~d},{ }^{3} J_{\mathrm{H}, \mathrm{H}}=6.8 \mathrm{~Hz}, 2 \mathrm{H}, \alpha-\underline{\mathrm{H}}_{2}\right), 4.05-4.32(\mathrm{~m}, 4 \mathrm{H}$, Fmoc-C ,

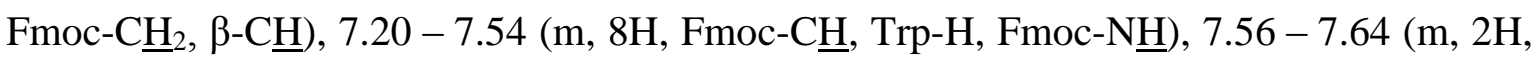
Fmoc-C마), $7.71\left(\mathrm{~d},{ }^{3} J_{\mathrm{H}, \mathrm{H}}=7.9 \mathrm{~Hz}, 1 \mathrm{H}, \operatorname{Trp}-\underline{\mathrm{H}}\right), 7.86\left(\mathrm{~d},{ }^{3} J_{\mathrm{H}, \mathrm{H}}=7.6 \mathrm{~Hz}, 2 \mathrm{H}\right.$, Fmoc-C $\left.\underline{\mathrm{H}}\right)$, $8.04\left(\mathrm{~d},{ }^{3} J_{\mathrm{H}, \mathrm{H}}=7.9 \mathrm{~Hz}, 1 \mathrm{H}, \mathrm{Trp}-\mathrm{H}\right)$.

MS (ESI, $\mathrm{MeOH}): \mathrm{m} / \mathrm{z}=563.2[\mathrm{M}+\mathrm{Na}]^{+} ; 1103.5[2 \mathrm{M}+\mathrm{Na}]^{+}$.

HR-MS (ESI, MeOH):

$\mathrm{C}_{32} \mathrm{H}_{32} \mathrm{~N}_{2} \mathrm{O}_{6} \mathrm{Na}[\mathrm{M}+\mathrm{Na}]^{+} \quad$ calc.: 563.2153

mesur.: 563.2148

(S)-3-((((9H-fluoren-9-yl)methoxy)carbonyl)amino)-4-(tert-butoxy)butanoic acid (Fmoc-D- $\left.\beta^{3}-h \operatorname{Ser}(t \mathrm{Bu})-\mathrm{OH}\right)$

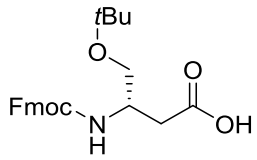

52

$\mathrm{C}_{23} \mathrm{H}_{27} \mathrm{NO}_{5}$

$397.19 \mathrm{~g} / \mathrm{mol}$

Following SOP 1 the synthesis was achieved starting with Fmoc-D-Ser( $t \mathrm{Bu})-\mathrm{OH}(5.00 \mathrm{~g}$, $13.00 \mathrm{mmol}, 1.00 \mathrm{eq})$ to yield the product $52(1.10 \mathrm{~g}, 2.77 \mathrm{mmol}, 21 \%)$ as a white solid.

${ }^{1} \mathbf{H}-\mathbf{N M R}\left(300 \mathrm{MHz},\left[\mathrm{D}_{6}\right] \mathrm{DMSO}\right): \delta(\mathrm{ppm})=1.10(\mathrm{~s}, 9 \mathrm{H}, \mathrm{O} \underline{\mathrm{Bu}}), 2.25$ - $2.39(\mathrm{~m}, 1 \mathrm{H} ; \alpha-$ $\left.\mathrm{C}_{2}\right), 2.43-2.57$ (m, 1H; $\alpha-\underline{\mathrm{H}}_{2}$, partially overlaid by solvent), $3.09-3.21$ (m, $1 \mathrm{H}, \gamma-$ $\left.\mathrm{C}_{2}\right), 3.25$ - $3.35\left(\mathrm{~m}, 1 \mathrm{H}, \gamma-\underline{\mathrm{H}}_{2}\right), 3.79$ - $3.93(\mathrm{~m}, 1 \mathrm{H}, \beta-\mathrm{C} \underline{\mathrm{H}}), 4.15$ - 4.37 (m, 3H, Fmoc- 


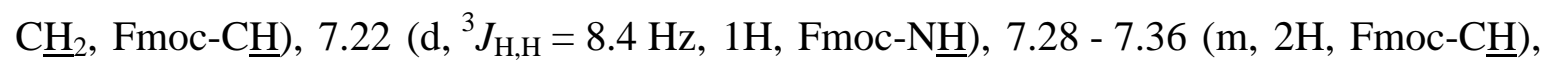

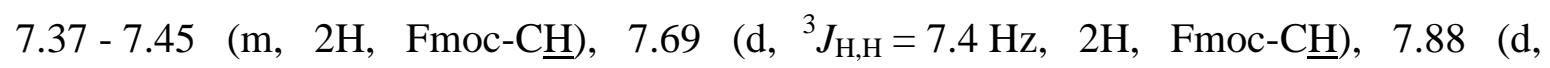
${ }^{3} J_{\mathrm{H}, \mathrm{H}}=7.5 \mathrm{~Hz}, 2 \mathrm{H}$, Fmoc-C $\left.\underline{\mathrm{H}}\right), 12.21\left(\mathrm{~s}_{\mathrm{br}}, 1 \mathrm{H}, \mathrm{COO} \underline{\mathrm{H}}\right)$.

${ }^{13}$ C-NMR (126 MHz, [D 6 DMSO): $\delta(p p m)=27.24,36.25,46.71,48.39,62.83,65.22$, 72.40, 119.93, 125.04, 126.87, 127.42, 140.54, 143.63, 143.75, 155.30, 172.33 .

MS (ESI, MeOH): m/z = 420.2[M+Na $]^{+}, 817.4[2 \mathrm{M}+\mathrm{Na}]^{+}, 1214.6[3 \mathrm{M}+\mathrm{Na}]^{+}$.

HR-MS (ESI, MeOH):

$\begin{array}{lll}\mathrm{C}_{23} \mathrm{H}_{27} \mathrm{NO}_{5}[\mathrm{M}+\mathrm{H}]^{+} & \text {calc.: } 398.1962 & \text { mesur.: 398.1966; } \\ \mathrm{C}_{23} \mathrm{H}_{27} \mathrm{NO}_{5} \mathrm{Na}[\mathrm{M}+\mathrm{Na}]^{+} & \text {calc.: } 420.1781 & \text { mesur.: 420.1783. }\end{array}$

(R)-3-(9-Fluorenylmethyloxycarbonyl)-amino-5-oxo-5-tritylamino-pentanoic acid (Fmoc-D- $\left.\beta^{3}-h A s n(T r t)-O H\right)$

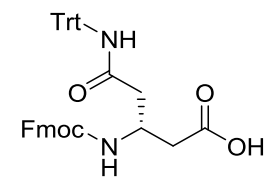

4

$\mathrm{C}_{39} \mathrm{H}_{34} \mathrm{~N}_{2} \mathrm{O}_{5}$

$610.25 \mathrm{~g} / \mathrm{mol}$

Following SOP 1 the synthesis was achieved starting with Fmoc-D-Asn(Trt)-OH (5.00 g, $8.37 \mathrm{mmol}, 1.00 \mathrm{eq})$ to yield the product $4(2.87 \mathrm{~g}, 4.71 \mathrm{mmol}, 56 \%)$ as a yellow solid.

${ }^{\mathbf{1}} \mathbf{H}-\mathrm{NMR}\left(300 \mathrm{MHz},\left[\mathrm{D}_{6}\right] \mathrm{DMSO}\right): \delta(\mathrm{ppm})=2.31-2.66\left(\mathrm{~m}, 4 \mathrm{H}, \mathrm{C}_{2}, \alpha-\underline{\mathrm{H}}_{2}\right.$, overlaid by solvent), $4.04-4.16$ (m, 1H, $\beta-\mathrm{C} \underline{\mathrm{H}}), 4.17-4.28$ (m, 2H, Fmoc- $\left.\underline{\mathrm{H}}_{2}\right), 4.29-4.41$ (m, 1H,

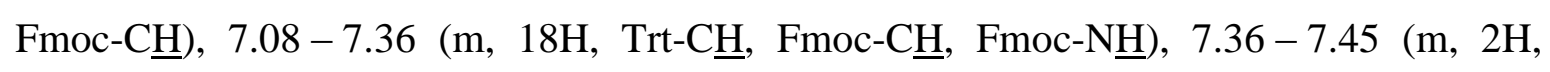

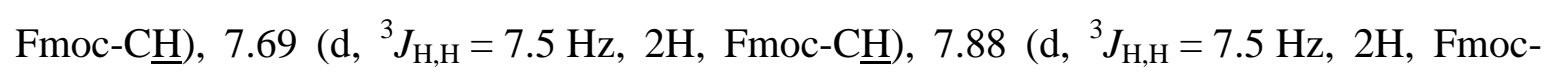

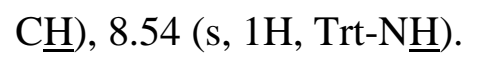

${ }^{13}$ C-NMR (125 MHz, [D 6 DMSO): $\delta(\mathrm{ppm})=40.85,46.71,65.34,69.31,119.87,124.94$, $125.04,125.08,126.10,126.85,126.88,127.18,127.39,127.96,128.07,128.34,128.66$, $129.00,140.47,143.60,143.63,144.59,154.91,169.38,172.12$. 
MS (ESI, MeOH): m/z = $611.3[\mathrm{M}+\mathrm{H}]^{+} ; 633.2[\mathrm{M}+\mathrm{Na}]^{+} ; 649.2[\mathrm{M}+\mathrm{K}]^{+}$.

HR-MS (ESI, MeOH):

$$
\begin{array}{lll}
\mathrm{C}_{39} \mathrm{H}_{35} \mathrm{~N}_{2} \mathrm{O}_{5}[\mathrm{M}+\mathrm{H}]^{+} & \text {calc.: 611.2540 } & \text { mesur.: 611.2529; } \\
\mathrm{C}_{39} \mathrm{H}_{34} \mathrm{~N}_{2} \mathrm{O}_{5} \mathrm{Na}[\mathrm{M}+\mathrm{Na}]^{+} & \text {calc.: 633.2360 } & \text { mesur.: 633.2340. }
\end{array}
$$

(R)-3-(((9H-Fluoren-9-yl)methoxy)carbonyl)amino)-4-cyanobutanoic acid (Fmoc-D- $\left.\beta^{3}-h A l a(C N)-O H\right)$

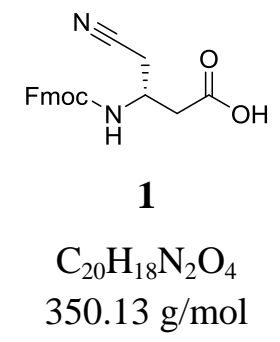

Fmoc-D- $\beta^{3}$-Asn(Trt)-OH $\quad(2.87 \mathrm{~g}, \quad 4.71 \mathrm{mmol}, \quad 1.00 \mathrm{eq})$ was stirred in $30 \mathrm{~mL}$ of TFA/EDT/ $\mathrm{H}_{2} \mathrm{O}(v / v / v$ 90:5:5) and the reaction was controlled by TLC. TFA was removed by a nitrogen stream and the crude product was precipitated, centrifuged and washed 3 times with diethyl ether. The dried intermediate product was suspended in ethyl acetate/acetonitrile (dry) (2:1) under an argon atmosphere. Propylphosphonic anhydride (50\% in DMF, T3P $\left.\mathrm{P}^{\circledR}\right)(2.75 \mathrm{~mL}, 9.42 \mathrm{mmol}, 2.00 \mathrm{eq})$ was added and the reaction mixture was stirred at $60{ }^{\circ} \mathrm{C}$ for $3 \mathrm{~h}$. The reaction mixture was poured in distilled water and extracted with ethyl acetate $(3 \times 100 \mathrm{~mL})$ to yield the product $(780 \mathrm{mg}, 2.23 \mathrm{mmol}, 47 \%)$ as a colourless solid.

${ }^{1}$ H-NMR (500 MHz, [D 6 ]DMSO): $\delta(\mathrm{ppm})=2.43-2.57\left(\mathrm{~m}, 2 \mathrm{H}, \alpha-\mathrm{C}_{2}\right.$, partially overlaid by solvent), $2.64-2.83\left(\mathrm{~m}, 2 \mathrm{H}, \underline{\mathrm{C}}_{2}\right), 3.98-4.08(\mathrm{~m}, 1 \mathrm{H}, \beta-\mathrm{C} \underline{\mathrm{H}}), 4.19-4.26(\mathrm{~m}, 1 \mathrm{H}$,

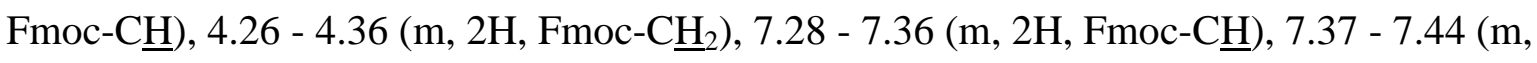

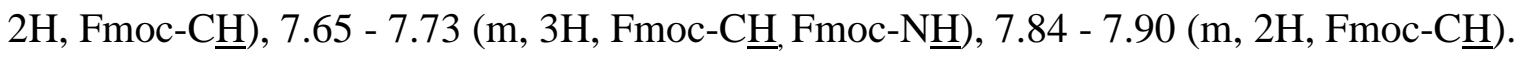

${ }^{13}$ C-NMR (126 MHz, [D 6 DMSO): $\delta(\mathrm{ppm})=22.54,38.09,44.93,46.62,65.51,66.29$, $118.22,120.03,125.11,127.01,127.56,140.66,143.69,143.74,155.32,171.51$.

MS (ESI, MeOH): m/z = $373.1[\mathrm{M}+\mathrm{Na}]^{+}, 349.1[\mathrm{M}-\mathrm{H}]^{-}$. 


\section{HR-MS (ESI, MeOH):}

$\begin{array}{lll}\mathrm{C}_{20} \mathrm{H}_{19} \mathrm{~N}_{2} \mathrm{O}_{4}[\mathrm{M}+\mathrm{H}]^{+} & \text {calc.: } 351.1339 & \text { mesur.: 351.1338; } \\ \mathrm{C}_{20} \mathrm{H}_{18} \mathrm{~N}_{2} \mathrm{O}_{4} \mathrm{Na}[\mathrm{M}+\mathrm{Na}]^{+} & \text {calc.: } 373.1159 & \text { mesur.: 373.1158; } \\ \mathrm{C}_{20} \mathrm{H}_{17} \mathrm{~N}_{2} \mathrm{O}_{4}[\mathrm{M}-\mathrm{H}]^{-} & \text {calc.: } 349.1194 & \text { mesur.: 349.1181. }\end{array}$

4-(4,6-Dimethoxy-1,3,5-triazin-2-yl)-4-methylmorpholin-4-ium 4-methylbenzenesulfonate $(D M T / N M M / T O S)^{[307]}$

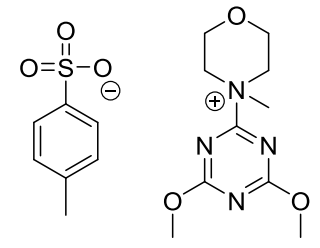

53

$\mathrm{C}_{18} \mathrm{H}_{26} \mathrm{~N}_{4} \mathrm{O}_{5} \mathrm{~S}$

$410.16 \mathrm{~g} / \mathrm{mol}$

DMT/NMM/TOS 53 was synthesised by following modified literature protocol. ${ }^{[307]} 2$ chloro-4,6-dimethoxy-1,3,5-triazine (CDMT) (2.00 g, $11.40 \mathrm{mmol}, 1.00 \mathrm{eq})$ were added to a cooled $\left(5^{\circ} \mathrm{C}\right)$ and stirred solution of $N$-methylmorpholine $(1.25 \mathrm{~mL}, 11.40 \mathrm{mmol}$, $1.00 \mathrm{eq})$, p-toluenesulfonic acid $(2.17 \mathrm{~g}, 11.40 \mathrm{mmol}, 1.00 \mathrm{eq})$ and sodium hydrogen carbonate $(2.87 \mathrm{~g}, 34.20 \mathrm{mmol}, 3.00 \mathrm{eq})$ in ACN (34 mL). The solution was stirred for $24 \mathrm{~h}$ at $5{ }^{\circ} \mathrm{C}$. After filtration and solvent removal by reduced pressure $\left(\mathrm{N}_{2}\right.$-rotary evaporator, $\mathrm{T} \leq 20^{\circ} \mathrm{C}$ ) the precipitation was washed with $\mathrm{THF}$, recrystallised from boiled diethyl ether with small amounts of $\mathrm{ACN}$ and crystallised at $-18{ }^{\circ} \mathrm{C}$. After filtration and drying $\mathbf{5 3}$ was obtained as a colourless solid (2.44 g, $59.10 \mathrm{mmol}, 52 \%)$.

${ }^{1} \mathbf{H}-\mathbf{N M R}\left(300 \mathrm{MHz},\left[\mathrm{D}_{3}\right] \mathrm{ACN}\right): \delta(\mathrm{ppm})=2.32\left(\mathrm{~s}, 3 \mathrm{H}, \mathrm{C}_{3_{3}}-\mathrm{C}_{6} \mathrm{H}_{4}-\right), 3.43\left(\mathrm{~s}, 3 \mathrm{H}, \mathrm{C}_{3}-\mathrm{N}\right)$, $3.65-3.77$ (m, 2H, -N-C $\left.\underline{H}_{2}-\mathrm{CH}_{2}-\mathrm{O}-\right)$, $3.78-3.89$ (m, 2H, -N-C $\left.\underline{\mathrm{H}}_{2}-\mathrm{CH}_{2}-\mathrm{O}-\right), 3.92-4.03$ (m, $\left.2 \mathrm{H},-\mathrm{N}_{-} \mathrm{CH}_{2}-\underline{\mathrm{C}}_{2}-\mathrm{O}-\right), 4.09$ (s, 6H, $\left.\mathrm{C}_{3}-\mathrm{O}\right), 4.35-4.46$ (m, 2H, -N-CH $\left.\mathrm{CH}_{2}-\underline{\mathrm{C}}_{2}-\mathrm{O}-\right)$, $7.09-7.23$ (m, $\left.2 \mathrm{H},-\mathrm{C}_{6} \underline{\mathrm{H}}_{4}^{-}\right), 7.52-7.63\left(\mathrm{~m}, 2 \mathrm{H},-\mathrm{C}_{6} \underline{\mathrm{H}}_{4}^{-}\right)$.

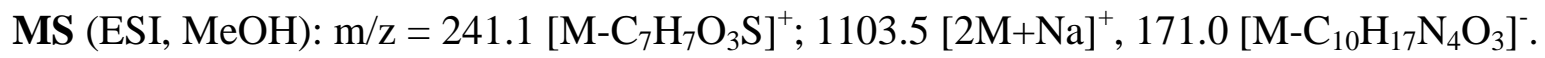

HR-MS (ESI, MeOH):

$$
\begin{array}{lll}
\mathrm{C}_{10} \mathrm{H}_{17} \mathrm{~N}_{4} \mathrm{O}_{3}\left[\mathrm{M}-\mathrm{C}_{7} \mathrm{H}_{7} \mathrm{O}_{3} \mathrm{~S}\right]^{+} & \text {calc.: } 241.1295 & \text { mesur.: 241.1298; } \\
\mathrm{C}_{7} \mathrm{H}_{7} \mathrm{O}_{3} \mathrm{~S}\left[\mathrm{M}-\mathrm{C}_{10} \mathrm{H}_{17} \mathrm{~N}_{4} \mathrm{O}_{3}\right]^{-} & \text {calc.: } 171.0121 & \text { mesur.: } 171.0122 .
\end{array}
$$




\subsubsection{2-[2'-(2'"-Aminophenoxy)ethoxy]-5-(benzyloxy)aniline}

\section{1,4-Bis(benzyloxy)benzene ${ }^{[308]}$}

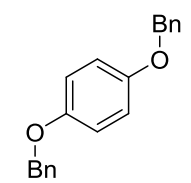

15

$\mathrm{C}_{20} \mathrm{H}_{18} \mathrm{O}_{2}$

$290.13 \mathrm{~g} / \mathrm{mol}$

Under argon atmosphere 1,4-hydroquinol (10.00 g, $90.90 \mathrm{mmol}, 1.00 \mathrm{eq})$ and $\mathrm{NaI}$ (14.30 g, $95.00 \mathrm{mmol}, 1.05 \mathrm{eq})$ were suspended in dry acetone $(50 \mathrm{~mL})$ at $0{ }^{\circ} \mathrm{C}$. Benzyl bromide (42.60 mL, $364 \mathrm{mmol}, 4.00 \mathrm{eq})$ was added slowly to the reaction mixture followed by $\mathrm{K}_{2} \mathrm{CO}_{3}(50.30 \mathrm{~g}, 364 \mathrm{mmol}, 4.00 \mathrm{eq})$. The reaction mixture was heated under reflux for $24 \mathrm{~h}$. The hot solution was poured in water $(500 \mathrm{~mL})$, the precipitation was filtered off and washed with water. The crude product was purified by recrystallization from boiling EtOH (if necessary with small portions of water). After filtration and drying under vacuum the product was given as colourless needles $(25.90 \mathrm{~g}, 89.20 \mathrm{mmol}, 98 \%)$.

${ }^{1}$ H-NMR $\left(300 \mathrm{MHz}, \mathrm{CDCl}_{3}\right): \delta(\mathrm{ppm})=5.05$ (s, 4H, -O-C $\left.\underline{\mathrm{H}}_{2}-\mathrm{Ph}\right), 6.96$ (s, 4H, $\left.\underline{\mathrm{Ph}}-(\mathrm{OBn})\right)$, $7.32-7.53\left(\mathrm{~m}, 10 \mathrm{H}, \mathrm{O}-\mathrm{CH}_{2}-\underline{\mathrm{Ph}}\right.$, solvent in signal).

${ }^{13} \mathbf{C}-\mathbf{N M R}\left(126 \mathrm{MHz}, \mathrm{CDCl}_{3}\right): \delta(\mathrm{ppm})=70.60,115.71,127.31,127.72,128.39,137.14$, 153.01

MS (EI, MeOH): m/z = $91.1\left[\mathrm{C}_{7} \mathrm{H}_{7}\right]^{+}, 290.2[\mathrm{M}]^{+\bullet}$.

MS (ESI, MeOH): m/z = 291.1 [M+H $]^{+}, 313.1[\mathrm{M}+\mathrm{Na}]^{+}$.

HR-MS (ESI, MeOH):

$\begin{array}{lll}\mathrm{C}_{20} \mathrm{H}_{19} \mathrm{O}_{2}[\mathrm{M}+\mathrm{H}]^{+} & \text {calc.: 291.1380 } & \text { mesur.: 291.1382; } \\ \mathrm{C}_{20} \mathrm{H}_{18} \mathrm{O}_{2} \mathrm{Na}[\mathrm{M}+\mathrm{Na}]^{+} & \text {calc.: } 313.1199 & \text { mesur.: } 313.1197 .\end{array}$




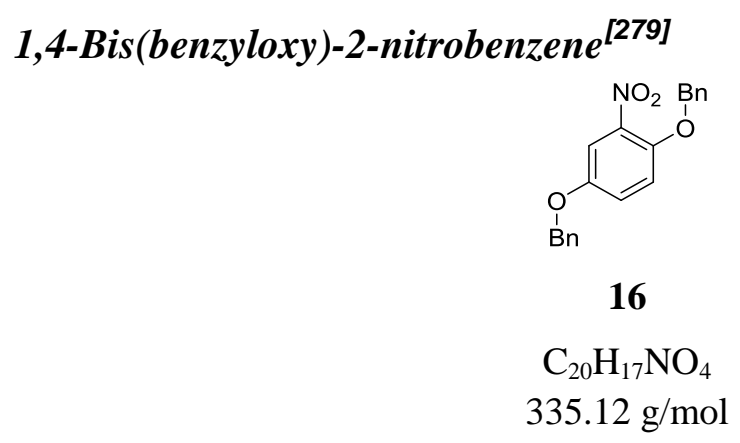

1,4-Bis(benzyloxy)benzene ( $25.90 \mathrm{~g}, 89.10 \mathrm{mmol}, 1.00 \mathrm{eq})$ was suspended in glacial acetic acid $(163 \mathrm{~mL})$ and cooled to $0{ }^{\circ} \mathrm{C}$. Nitric acid $(65 \%, 8.55 \mathrm{~mL}, 178.00 \mathrm{mmol}, 2.00 \mathrm{eq})$ was added slowly and the solution was stirred rapidly at $0{ }^{\circ} \mathrm{C}$ for $3 \mathrm{~h}$. When the solution was frozen it was melted in a sonication bath. Afterwards the reaction was poured in water, filtered off and washed with water. The product was filtered off with DCM on Silical gel and dried by lyophilisation to yield the product $(29.20 \mathrm{~g}, 86.90 \mathrm{mmol}, 98 \%)$ as a yellow solid.

TLC (pentane/DCM, 3:1): $R_{f}=0.63$.

${ }^{1} \mathbf{H}-\mathrm{NMR}\left(300 \mathrm{MHz}, \mathrm{CDCl}_{3}\right): \delta(\mathrm{ppm})=5.03\left(\mathrm{~s}, 2 \mathrm{H},-\mathrm{O}-\mathrm{C}_{2}{ }_{2} \mathrm{Ph}\right) .5 .16\left(\mathrm{~s}, 2 \mathrm{H},-\mathrm{O}-\underline{\mathrm{H}}_{2} 2^{-}\right.$ $\mathrm{Ph}), 7.03\left(\mathrm{~d},{ }^{3} J_{\mathrm{H}, \mathrm{H}}=9.2 \mathrm{~Hz}, 1 \mathrm{H}, \underline{\mathrm{Ph}}-(\mathrm{OBn})\right), 7.11\left(\mathrm{dd},{ }^{3} J_{\mathrm{H}, \mathrm{H}}=9.2,3.0 \mathrm{~Hz}, 1 \mathrm{H}, \underline{\mathrm{Ph}}-(\mathrm{OBn})\right)$, $7.21-7.53\left(\mathrm{~m}, 11 \mathrm{H}, \mathrm{O}-\mathrm{CH}_{2}-\underline{\mathrm{Ph}}, \underline{\mathrm{Ph}}-(\mathrm{OBn})\right)$.

${ }^{13}$ C-NMR $\left(75 \mathrm{MHz}, \mathrm{CDCl}_{3}\right): \delta(\mathrm{ppm})=70.83,71.98,111.12,117.14,121.39,127.04$, $127.40,127.47,128.10,128.25,128.58,128.63,135.80,135.91,140.19,146.27,152.20$.

MS (ESI, MeOH): m/z = 353.2 $\left[\mathrm{M}+\mathrm{NH}_{4}\right]^{+}, 358.1[\mathrm{M}+\mathrm{Na}]^{+}$.

HR-MS (ESI, MeOH):

$\begin{array}{lll}\mathrm{C}_{20} \mathrm{H}_{21} \mathrm{~N}_{2} \mathrm{O}_{4}\left[\mathrm{M}+\mathrm{NH}_{4}\right]^{+} & \text {calc.: } 353.1496 & \text { mesur.: } 353.1489 ; \\ \mathrm{C}_{20} \mathrm{H}_{17} \mathrm{NO}_{4} \mathrm{Na}[\mathrm{M}+\mathrm{Na}]^{+} & \text {calc.: } 358.1050 & \text { mesur.: } 358.1043 .\end{array}$




\section{4-Benzyloxy-2-nitrophenol ${ }^{[279]}$}

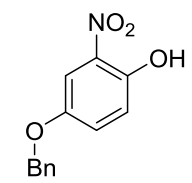

12

$\mathrm{C}_{13} \mathrm{H}_{11} \mathrm{NO}_{4}$

$245.07 \mathrm{~g} / \mathrm{mol}$

1,4-Bis(benzyloxy)benzene $(25.00 \mathrm{~g}, 74.60 \mathrm{mmol}, 1.00 \mathrm{eq})$ was suspended in dry chloroform $\left(700 \mathrm{~mL}, 4 \AA\right.$ molecular sieve) and cooled to $0{ }^{\circ} \mathrm{C}$. Aluminium chloride (10.40 g, $78.30 \mathrm{mmol}, 1.05 \mathrm{eq})$ was added and the solution was stirred for $10-20 \mathrm{~min}$. The reaction process was monitored by TLC. The reaction was quenched with $10 \% \mathrm{HCl}$ $(700 \mathrm{~mL})$, extracted with DCM $(3 \times 300 \mathrm{~mL})$ and filtered. The solvent of the combined organic phases was removed under reduced pressure. The desired product was obtained after purification by flash-column chromatography (pentane/DCM, 7:1; alternatively $100 \%$ toluene) as a yellow solid (13.70 g, $56.00 \mathrm{mmol}, 75 \%)$.

TLC (pentane/DCM, 3:1): $R_{f}=0.83$.

${ }^{1}$ H-NMR $\left(300 \mathrm{MHz},\left[\mathrm{D}_{6}\right] \mathrm{DMSO}\right): \delta(\mathrm{ppm})=5.10 \quad\left(\mathrm{~s}, \quad 2 \mathrm{H},-\mathrm{O}-\mathrm{CH}_{2}-\mathrm{Ph}\right), 7.09$ (d, $\left.{ }^{3} J_{\mathrm{H}, \mathrm{H}}=9.1 \mathrm{~Hz}, 1 \mathrm{H}, \underline{\mathrm{Ph}}-(\mathrm{OBn})\right), 7.25-7.48\left(\mathrm{~m}, 6 \mathrm{H}, \mathrm{O}-\mathrm{CH}_{2}-\underline{\mathrm{Ph}}, \underline{\mathrm{Ph}}-(\mathrm{OBn})\right), 7.51$ (d, $\left.{ }^{3} J_{\mathrm{H}, \mathrm{H}}=3.1 \mathrm{~Hz}, 1 \mathrm{H}, \underline{\mathrm{Ph}}-(\mathrm{OBn})\right), 10.47$ (s, 1H, ArO $\left.\underline{\mathrm{H}}\right)$.

${ }^{13}$ C-NMR $\left(75 \mathrm{MHz},\left[\mathrm{D}_{6}\right] \mathrm{DMSO}\right): \delta(\mathrm{ppm})=70.57,109.60,120.68,124.54,128.22$, 128.40, 128.89, 136.47, 137.04, 147.10, 150.93 .

MS (ESI, $\mathrm{MeOH}): \mathrm{m} / \mathrm{z}=244.2[\mathrm{M}-\mathrm{H}]^{-}$.

HR-MS (ESI, MeOH):
$\mathrm{C}_{13} \mathrm{H}_{10} \mathrm{NO}_{4}[\mathrm{M}-\mathrm{H}]^{-}$
calc.: 244.0615
mesur.: 244.0618. 


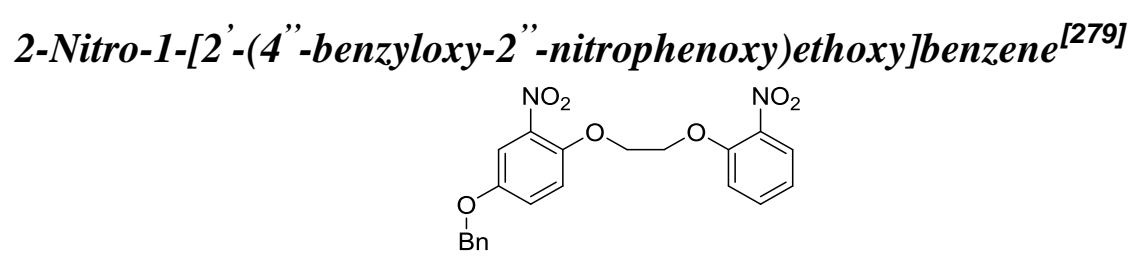

18

$\mathrm{C}_{21} \mathrm{H}_{18} \mathrm{~N}_{2} \mathrm{O}_{7}$

$410.11 \mathrm{~g} / \mathrm{mol}$

4-Benzyloxy-2-nitrophenol $(6.00 \mathrm{~g}, 24.50 \mathrm{mmol}, 1.00 \mathrm{eq})$ and dry potassium carbonate (3.38 g, $24.50 \mathrm{mmol}, 1.00 \mathrm{eq})$ were dissolved in dry DMF (14 mL) and heated to $120^{\circ} \mathrm{C}$ under argon atmosphere. 1-(2-Bromoethoxy)-2-nitrobenzene (8.85 g, $36.00 \mathrm{mmol}, 1.47 \mathrm{eq})$ was added and the mixture was stirred for 5-10 min. The reaction process was monitored by TLC. Afterwards, the mixture was diluted with saturated sodium bicarbonate solution, extracted with $\mathrm{DCM}$ and dried over $\mathrm{MgSO}_{4}$. The solvent was evaporated under reduced pressure and the crude product was purified by flash column chromatography (pentane/EE/DCM, 2:1:1). The product was obtained as yellow solid $(9.70 \mathrm{~g}, 23.60 \mathrm{mmol}$, $97 \%)$.

TLC (pentane/EE 2:1): $R_{f}=0.63$.

${ }^{\mathbf{1}} \mathbf{H}-\mathrm{NMR}\left(300 \mathrm{MHz},\left[\mathrm{D}_{6}\right] \mathrm{DMSO}\right): \delta(\mathrm{ppm})=4.44-4.54$ (m, 4H, -O-C $\left.\underline{\mathrm{H}}_{2} \underline{\mathrm{CH}}_{2}-\mathrm{O}-\right), 5.14$ (s, $\left.2 \mathrm{H},-\mathrm{O}-\underline{\mathrm{H}}_{2}-\mathrm{Ph}\right), 7.07-7.18\left(\mathrm{~m}, 1 \mathrm{H}, \underline{\mathrm{Ph}}-\mathrm{NO}_{2}\right), 7.26-7.50\left(\mathrm{~m}, 8 \mathrm{H}, \mathrm{O}-\mathrm{CH}_{2}-\underline{\mathrm{Ph}}, \underline{\mathrm{Ph}}-\mathrm{NO}_{2}\right.$, $\underline{\mathrm{Ph}}-(\mathrm{OBn})), 7.53\left(\mathrm{~d},{ }^{3} \mathrm{~J}_{\mathrm{H}, \mathrm{H}}=2.9 \mathrm{~Hz}, 1 \mathrm{H}, \underline{\mathrm{Ph}}-\mathrm{NO}_{2}\right), 7.60-7.69\left(\mathrm{~m}, 1 \mathrm{H},-\underline{\mathrm{Ph}}-\mathrm{NO}_{2}\right), 7.85(\mathrm{dd}$, $\left.{ }^{3} J_{\mathrm{H}, \mathrm{H}}=8.1 \mathrm{~Hz}, 1.7 \mathrm{~Hz}, 1 \mathrm{H},-\underline{\mathrm{Ph}}-\mathrm{NO}_{2}\right)$.

${ }^{13}$ C-NMR (75 MHz, [D $]$ DMSO): $\delta(p p m)=68.64,69.26,70.64,110.96,115.99,117.99$, $121.40,121.59,125.34,128.25,128.45,128.92$, 134.77, 136.91, 140.20, 140.50, 145.57, $151.42,152.38$.

MS (ESI, MeOH): m/z = 433.1 $[\mathrm{M}+\mathrm{Na}]^{+}, 843.2[2 \mathrm{M}+\mathrm{Na}]^{+}$.

HR-MS (ESI, MeOH):

$\mathrm{C}_{21} \mathrm{H}_{18} \mathrm{~N}_{2} \mathrm{O}_{7} \mathrm{Na}[\mathrm{M}+\mathrm{Na}]^{+} \quad$ calc.: $433.1006 \quad$ mesur.: 433.1000 . 


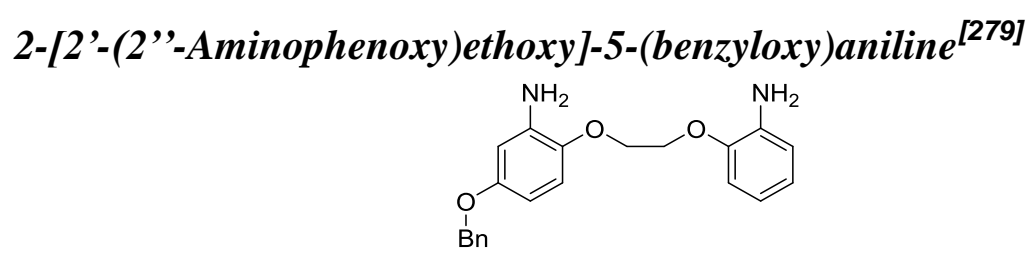

19

$\mathrm{C}_{21} \mathrm{H}_{22} \mathrm{~N}_{2} \mathrm{O}_{3}$

$350.16 \mathrm{~g} / \mathrm{mol}$

Ammonium chloride (9.65 g, 180.40 mmol, 6.20 eq) and 2-nitro-1-[2'-(4' '-benzyloxy-2''nitrophenoxy)ethoxy]benzene $(12.00 \mathrm{~g}, 29.20 \mathrm{mmol}, 1.00 \mathrm{eq})$ were dissolved in acetone $/ \mathrm{H}_{2} \mathrm{O}$ (3:1) under argon atmosphere in a miniclave (BÜCHI, Uster, Switzerland). Iron powder (12.60 g, $225.20 \mathrm{mmol}, 7.70 \mathrm{eq})$ was added. The mixture was stirred for 1 $4 \mathrm{~h}$ at $70{ }^{\circ} \mathrm{C}$. The solvent was evaporated under reduced pressure and the crude product purified by flash column chromatography (pentane/EE, 10:1 $\rightarrow 0: 1$ ). The product was obtained as brown solid (4.30 g, $16.70 \mathrm{mmol}, 98 \%)$.

${ }^{1}$ H-NMR (300 MHz, [D 6 ]DMSO): $\delta(\mathrm{ppm})=4.15-4.29$ (m, 4H, -O-C $\underline{\mathrm{H}}_{2} \mathrm{C}_{2}-\mathrm{O}-$ ), 4.75 (s, $\left.4 \mathrm{H},-\mathrm{O}-\mathrm{C}_{2}-\mathrm{Ph}, \mathrm{Ph}-\underline{\mathrm{N}}_{2}\right), 4.96\left(\mathrm{~s}, 2 \mathrm{H}, \mathrm{Ph}-\underline{N}_{2}\right), 6.16\left(\mathrm{dd},{ }^{3} J_{\mathrm{H}, \mathrm{H}}=8.6 \mathrm{~Hz}, 3.0 \mathrm{~Hz}, 1 \mathrm{H}\right.$, $\left.\underline{\mathrm{Ph}}-\mathrm{NH}_{2}\right), 6.37\left(\mathrm{~d},{ }^{3} \mathrm{~J}_{\mathrm{H}, \mathrm{H}}=3.0 \mathrm{~Hz}, 1 \mathrm{H}, \underline{\mathrm{Ph}}-\mathrm{NH}_{2}\right), 6.46-6.59\left(\mathrm{~m}, 1 \mathrm{H}, \underline{\mathrm{Ph}}-\mathrm{NH}_{2}\right), 6.64-6.81$ $\left(\mathrm{m}, 3 \mathrm{H}, \underline{\mathrm{Ph}}-\mathrm{NH}_{2}\right), 6.85\left(\mathrm{dd},{ }^{3} J_{\mathrm{H}, \mathrm{H}}=8.0 \mathrm{~Hz}, 1.3 \mathrm{~Hz}, 1 \mathrm{H}, \underline{\mathrm{Ph}}-\mathrm{NH}_{2}\right), 7.25-7.47$ (m, 5H, O$\left.\mathrm{CH}_{2}-\underline{\mathrm{Ph}}\right)$.

${ }^{13}$ C-NMR (75 MHz, [D $]$ DMSO): $\delta(\mathrm{ppm})=67.72,68.68,69.70,101.68,102.08,113.15$, 114.63 , 114.81, 116.68, 121.91, 127.91, 128.04, 128.79, 138.11, 138.62, 140.02, 140.39, $145.85,154.13$.

MS (ESI, MeOH): m/z = $351.2[\mathrm{M}+\mathrm{H}]^{+}, 373.2[\mathrm{M}+\mathrm{Na}]^{+}$.

HR-MS (ESI, MeOH):

$\begin{array}{lll}\mathrm{C}_{21} \mathrm{H}_{23} \mathrm{~N}_{2} \mathrm{O}_{3}[\mathrm{M}+\mathrm{H}]^{+} & \text {calc.: } 351.1703 & \text { mesur.: 351.1702; } \\ \mathrm{C}_{21} \mathrm{H}_{22} \mathrm{~N}_{2} \mathrm{O}_{3} \mathrm{Na}[\mathrm{M}+\mathrm{Na}]^{+} & \text {calc.: } 373.1523 & \text { mesur.: 373.1512. }\end{array}$




\subsubsection{Base-labile BAPTA-based $\mathrm{Ca}^{2+}$-sensor}

Dimethyl 2,2'-((5-(benzyloxy)-2-(2-(2-(bis(2-methoxy-2oxoethyl)amino)phenoxy)ethoxy)phenyl)azanediyl)diacetate<smiles>COC(=O)CN(CC(=O)OC)c1ccccc1OCCOc1ccc(OCc2ccccc2)cc1N(CC(=O)OC)CC(=O)OC</smiles>

21

$\mathrm{C}_{33} \mathrm{H}_{38} \mathrm{~N}_{2} \mathrm{O}_{11}$

$638.25 \mathrm{~g} / \mathrm{mol}$

2-[2'-(2"'-Aminophenoxy)ethoxy]-5-(benzyloxy)aniline $\quad(3.80 \mathrm{~g}, 10.84 \mathrm{mmol}, 1.00 \mathrm{eq})$, sodium iodide $(4.47 \mathrm{~g}, 29.82 \mathrm{mmol}, 2.75 \mathrm{eq})$ and 1,8-bis(dimethylamino)naphthalene $(15.83 \mathrm{~g}, 73.85 \mathrm{mmol}, 6.81 \mathrm{eq})$ were dissolved in dry $\mathrm{ACN}(21 \mathrm{~mL})$ under argon atmosphere. Methyl bromoacetate $(9.25 \mathrm{~mL}, 97.71 \mathrm{mmol}, 9.01 \mathrm{eq})$ was added slowly and the solution was stirred at $80{ }^{\circ} \mathrm{C}$ for $24 \mathrm{~h}$. The solvent was evaporated under reduced pressure and the residue was dissolved in toluene and filtered. The organic solvent was washed with diluted $\mathrm{HCl}$ and brine solution until no 1,8-bis(dimethylamino)naphthalene remain in the organic phase (TLC). The solvent was evaporated under reduced pressure, the crude product was purified by recrystallisation (Petroleum ether $(60-80) / E E$ ) and obtained as white solid $(5.47 \mathrm{~g}, 8.56 \mathrm{mmol}, 79 \%)$.

${ }^{1} \mathbf{H}-N M R\left(300 \mathrm{MHz},\left[\mathrm{D}_{6}\right] \mathrm{DMSO}\right): \delta(\mathrm{ppm})=3.47-3.53(\mathrm{~m}, 12 \mathrm{H},-\mathrm{COOMe}), 4.08-4.16$

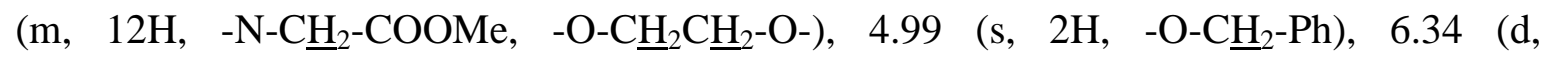
$\left.{ }^{3} J_{\mathrm{H}, \mathrm{H}}=2.9 \mathrm{~Hz}, 1 \mathrm{H}, \underline{\mathrm{Ph}}-\mathrm{N}-\left(\mathrm{CH}_{2}\right)_{2}-\right), 6.50\left(\mathrm{dd},{ }^{3} J_{\mathrm{H}, \mathrm{H}}=8.8 \mathrm{~Hz}, 2.9 \mathrm{~Hz}, 1 \mathrm{H}, \underline{\mathrm{Ph}}-\mathrm{N}-\left(\mathrm{CH}_{2}\right)_{2}-\right)$, $6.68-6.77\left(\mathrm{~m}, 1 \mathrm{H}, \underline{\mathrm{Ph}}-\mathrm{N}-\left(\mathrm{CH}_{2}\right)_{2^{-}}\right), 6.79-6.97\left(\mathrm{~m}, 4 \mathrm{H}, \underline{\mathrm{Ph}}-\mathrm{N}-\left(\mathrm{CH}_{2}\right)_{2}-\right), 7.28-7.45(\mathrm{~m}$, $\left.5 \mathrm{H}, \mathrm{O}-\mathrm{CH}_{2}-\underline{\mathrm{Ph}}\right)$.

${ }^{13}$ C-NMR (75 MHz, [D $]$ DMSO): $\delta(\mathrm{ppm})=51.19,51.23,53.00,67.05,67.73,69.47$, $105.51,106.07,113.32,114.59$, 118.02, 120.98, 121.21, 127.57, 127.64, 128.31, 137.32, 138.69, 139.85, 143.88, 149.60, 152.89, 171.13, 171.21.

MS (ESI, MeOH): m/z = 661.2[M+Na] $]^{+}$ 
HR-MS (ESI, MeOH):

$$
\begin{array}{lll}
\mathrm{C}_{33} \mathrm{H}_{39} \mathrm{~N}_{2} \mathrm{O}_{11}[\mathrm{M}+\mathrm{H}]^{+} & \text {calc.: 639.2548 } & \text { mesur.: 639.2549; } \\
\mathrm{C}_{33} \mathrm{H}_{38} \mathrm{~N}_{2} \mathrm{O}_{11} \mathrm{Na}[\mathrm{M}+\mathrm{Na}]^{+} & \text {calc.: } 661.2368 & \text { mesur.: 6661.2362. }
\end{array}
$$

Dimethyl 2,2'-((5-(benzyloxy)-2-(2-(2-(bis(2-methoxy-2oxoethyl)amino)phenoxy)ethoxy)-4-formylphenyl)azanediyl)diacetate<smiles>COC(=O)CN(CC(=O)OC)c1ccccc1OCCOc1cc(C=O)c(Br)cc1N(CC(=O)OC)CC(=O)OC</smiles>

22

$\mathrm{C}_{34} \mathrm{H}_{38} \mathrm{~N}_{2} \mathrm{O}_{12}$

$666.24 \mathrm{~g} / \mathrm{mol}$

Dimethyl 2,2'-((5-(benzyloxy)-2-(2-(2-(bis(2-methoxy-2-oxoethyl)amino)phenoxy)ethoxy)phenyl)azanediyl)diacetate $(5.47 \mathrm{~g}, 8.56 \mathrm{mmol}, 1.00 \mathrm{eq})$ was dissolved in dry DMF (42 mL) under argon atmosphere. Pyridine (6.91 mL, $85.65 \mathrm{mmol}, 10.00 \mathrm{eq})$ was added and the reaction mixture was cooled to $0{ }^{\circ} \mathrm{C}$. Phosphoryl chloride $(6.41 \mathrm{~mL}, 68.52 \mathrm{mmol}$, $8.00 \mathrm{eq}$ ) was added and stirred at $0{ }^{\circ} \mathrm{C}$ for $1.5 \mathrm{~h}$. The reaction was quenched with cold $1 \mathrm{M}$ $\mathrm{KOH}$ solution, the crude product was extracted with DCM $(3 \times 100 \mathrm{~mL})$, the organic phase was washed with brine and dried over $\mathrm{MgSO}_{4}$. The solvent was evaporated under reduced pressure and the crude product was purified by filtration over $\mathrm{SiO}_{2}$ with $\mathrm{EE}$. The product was obtained as yellow solid (5.16 g, $7.74 \mathrm{mmol}, 90 \%)$.

${ }^{1}$ H-NMR (300 MHz, [D 6 DMSO): $\delta(\mathrm{ppm})=3.47-3.54(\mathrm{~m}, 12 \mathrm{H},-\mathrm{COOMe}), 4.11(\mathrm{~m}$, $8 \mathrm{H},-\mathrm{N}-\underline{\mathrm{H}}_{2}-\mathrm{COOMe}$ ), 4.25 (s, 4H, -O-C $\underline{\mathrm{H}}_{2} \underline{\mathrm{C}}_{2}-\mathrm{O}-$ ), 5.20 (s, $2 \mathrm{H},-\mathrm{O}-\mathrm{C}_{2}-\mathrm{Ph}$ ), 6.35 (s, $1 \mathrm{H}$, $\left.\underline{\mathrm{Ph}}-\mathrm{N}-\left(\mathrm{CH}_{2}\right)_{2^{-}}\right), 6.67-6.75$ (m, 1H, $\left.\underline{\mathrm{Ph}}-\mathrm{N}-\left(\mathrm{CH}_{2}\right)_{2^{-}}\right), 6.80-6.95$ (m, 3H, $\left.\underline{\mathrm{Ph}}-\mathrm{N}-\left(\mathrm{CH}_{2}\right)_{2^{-}}\right)$, 7.15 (s, 1H, $\left.\underline{\mathrm{Ph}}-\mathrm{N}-\left(\mathrm{CH}_{2}\right)_{2}-\right), 7.30-7.47$ (m, 5H, O-CH$\left.{ }^{-}-\underline{\mathrm{Ph}}\right), 10.17$ (s, 1H, - $\underline{\mathrm{H}}$ - $)$ ).

${ }^{13}$ C-NMR (75 MHz, [D 6 DMSO): $\delta(\mathrm{ppm})=51.18,51.51,52.97,53.54,66.64,67.49$, 70.44, 102.10, 109.97, 113.09, 116.32, 117.82, 120.98, 121.12, 127.44, 127.88, 128.47, $136.64,138.70,142.94,146.05,149.46,156.89,170.51,171.25,186.05$.

MS (ESI, MeOH): m/z = $689.2[\mathrm{M}+\mathrm{Na}]^{+}$. 
HR-MS (ESI, MeOH):

$\begin{array}{lll}\mathrm{C}_{34} \mathrm{H}_{39} \mathrm{~N}_{2} \mathrm{O}_{12}[\mathrm{M}+\mathrm{H}]^{+} & \text {calc.: 667.2498 } & \text { mesur.: 667.2499; } \\ \mathrm{C}_{34} \mathrm{H}_{38} \mathrm{~N}_{2} \mathrm{O}_{12} \mathrm{Na}[\mathrm{M}+\mathrm{Na}]^{+} & \text {calc.: } 689.2317 & \text { mesur.: 689.2312. }\end{array}$

\section{Dimethyl 2,2'-((2-(2-(2-(bis(2-methoxy-2-oxoethyl)amino)-5-formyl-4- hydroxyphenoxy)ethoxy)phenyl)azanediyl)diacetate}<smiles>COC(=O)CN(CC(=O)OC)c1ccccc1OCCOc1cc(C=O)c(O)c(O)c1N(CC(=O)OC)CC(=O)OC</smiles>

23

$\mathrm{C}_{27} \mathrm{H}_{32} \mathrm{~N}_{2} \mathrm{O}_{12}$

$576.20 \mathrm{~g} / \mathrm{mol}$

Dimethyl 2,2'-((5-(benzyloxy)-2-(2-(2-(bis(2-methoxy-2-oxoethyl)amino)phenoxy)ethoxy)-4-formylphenyl)azanediyl)diacetate (4.80 g, $7.20 \mathrm{mmol}, 1.00 \mathrm{eq})$ was dissolved in acetic acid $(14.70 \mathrm{~mL} / \mathrm{g}$ educt) and $10 \% \mathrm{Pd} / \mathrm{C}(294.10 \mathrm{mg} / \mathrm{g}$ educt $)$ was added. The solution was stirred for up to $3 \mathrm{~h}$ under $\mathrm{H}_{2}$ atmosphere (room pressure). The reaction solution was filtered over celite and the solvent was evaporated under reduced pressure. The crude product was purified by filtration over $\mathrm{SiO}_{2}$ with EE. The product was obtained as white solid (3.16 g, $5.49 \mathrm{mmol}, 76 \%)$.

${ }^{\mathbf{1}} \mathbf{H}-\mathbf{N M R}\left(300 \mathrm{MHz},\left[\mathrm{D}_{6}\right] \mathrm{CDCl} 3\right): \delta(\mathrm{ppm})=3.48-3.59(\mathrm{~m}, 12 \mathrm{H},-\mathrm{COOMe}), 4.07-4.20$ (m, 12H, -N-C $\left.\underline{\mathrm{H}}_{2}-\mathrm{COOMe},-\mathrm{O}-\underline{\mathrm{CH}}_{2} \underline{\mathrm{CH}}_{2}-\mathrm{O}-\right), 6.08$ (s, 1H, $\left.\underline{\mathrm{Ph}}-\mathrm{N}-\left(\mathrm{CH}_{2}\right)_{2}{ }^{-}\right), 6.74-6.92$ (m, $\left.5 \mathrm{H}, \underline{\mathrm{Ph}}-\mathrm{N}-\left(\mathrm{CH}_{2}\right)_{2}-\right), 9.55$ (s, 1H, Ph-Oㅂ) $), 11.14$ (s, 1H, - $\underline{\mathrm{HO}}$ ).

MS (ESI, MeOH): m/z = 577.2 $[\mathrm{M}+\mathrm{H}]^{+}, 599.2[\mathrm{M}+\mathrm{Na}]^{+}$.

HR-MS (ESI, MeOH):

$\begin{array}{lll}\mathrm{C}_{27} \mathrm{H}_{33} \mathrm{~N}_{2} \mathrm{O}_{12}[\mathrm{M}+\mathrm{H}]^{+} & \text {calc.: 577.2028 } & \text { mesur.: 577.2028; } \\ \mathrm{C}_{27} \mathrm{H}_{32} \mathrm{~N}_{2} \mathrm{O}_{12} \mathrm{Na}[\mathrm{M}+\mathrm{Na}]^{+} & \text {calc.: } 599.1847 & \text { mesur.: 599.1852. }\end{array}$


Dimethyl 2,2'-((3-(1H-benzoimidazol-2-yl)-6-(2-(2-(bis(2-methoxy-2oxoethyl)amino)phenoxy)ethoxy)-2-imino-2H-chromen-7-yl)azanediyl)diacetate<smiles>COC(=O)CN(CC(=O)OC)c1ccccc1OCCOc1cc2cc(-c3nc4ccccc4[nH]3)c(=N)oc2cc1N(CC(=O)OC)CC(=O)OC</smiles>

8

$\mathrm{C}_{36} \mathrm{H}_{37} \mathrm{~N}_{5} \mathrm{O}_{11}$

$715.25 \mathrm{~g} / \mathrm{mol}$

Piperidine (1.70 mL, $1.73 \mathrm{mmol}, 1.00 \mathrm{eq})$ was added to a solution of dimethyl 2,2'-((2-(2(2-(bis(2-methoxy-2-oxoethyl)amino)-5-formyl-4-

hydroxyphenoxy)ethoxy)phenyl)azanediyl)diacetate (273 mg, $1.74 \mathrm{mmol}, 1.00 \mathrm{eq}$ ) in dry $\mathrm{MeOH}(10 \mathrm{~mL})$. Then 2-cyanomethylbenzimidazole $(1.00 \mathrm{~g}, 1.73 \mathrm{mmol}, 1.00 \mathrm{eq})$ was added and the solution was stirred overnight under argon atmosphere at RT. The solvent was removed in vacuo, the product was obtained as yellow solid and used without further purifications.

MS (ESI, $\mathrm{MeOH}): \mathrm{m} / \mathrm{z}=716.2[\mathrm{M}+\mathrm{H}]^{+}, 738.2[\mathrm{M}+\mathrm{Na}]^{+}$.

HR-MS (ESI, MeOH):
$\mathrm{C}_{36} \mathrm{H}_{38} \mathrm{~N}_{5} \mathrm{O}_{11}[\mathrm{M}+\mathrm{H}]^{+}$
calc.: 716.2562
mesur.: 716.2567;
$\mathrm{C}_{36} \mathrm{H}_{37} \mathrm{~N}_{5} \mathrm{O}_{11} \mathrm{Na}[\mathrm{M}+\mathrm{Na}]^{+} \quad$ calc.: 738.2382
mesur.: 738.2383 . 
Dimethyl 2,2'-((2-((2-((2-aminoethyl)disulfaneyl)ethyl)imino)-3-(1Hbenzoimidazol-2-yl)-6-(2-(2-(bis(2-methoxy-2-oxoethyl)amino)phenoxy)ethoxy)-2H-chromen-7-yl)azanediyl)(Z/E)-diacetate

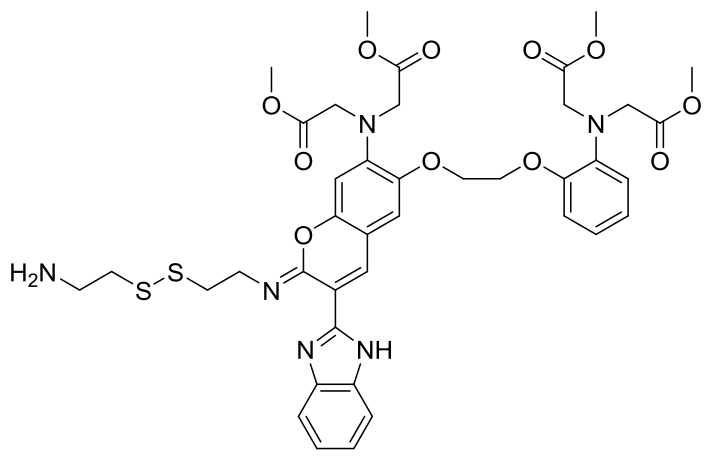

28

$\mathrm{C}_{40} \mathrm{H}_{46} \mathrm{~N}_{6} \mathrm{O}_{11} \mathrm{~S}_{2}$

$850.27 \mathrm{~g} / \mathrm{mol}$

Following SOP 8 the synthesis was achieved by starting with $\mathbf{8}$. The product $\mathbf{2 8}$ was obtained as a yellow solid.

MS (ESI, MeOH): m/z = 426.1 $[\mathrm{M}+2 \mathrm{H}]^{2+} 851.2[\mathrm{M}+\mathrm{H}]^{+}$.

HR-MS (ESI, MeOH):
$\mathrm{C}_{40} \mathrm{H}_{48} \mathrm{~N}_{6} \mathrm{O}_{11} \mathrm{~S}_{2}[\mathrm{M}+2 \mathrm{H}]^{2+} \quad$ calc.: 426.1406
mesur.: 426.1410;
$\mathrm{C}_{40} \mathrm{H}_{47} \mathrm{~N}_{6} \mathrm{O}_{11} \mathrm{~S}_{2}[\mathrm{M}+\mathrm{H}]^{+} \quad$ calc.: 851.2739
mesur.: 851.2740.

Dimethyl 2,2'-((3-(1H-benzoimidazol-2-yl)-6-(2-(2-(bis(2-methoxy-2oxoethyl)amino)phenoxy)ethoxy)-2-((2-(pyridin-2-yldisulfaneyl)ethyl)imino)2H-chromen-7-yl)azanediyl)(Z/E)-diacetate<smiles>COC(=O)CN(CC(=O)OC)c1ccccc1OCCOc1cc2cc(-c3nc4ccccc4[nH]3)/c(=N/CCSSc3ccccn3)oc2cc1N(CC(=O)OC)CC(=O)OC</smiles>

27

$\mathrm{C}_{43} \mathrm{H}_{44} \mathrm{~N}_{6} \mathrm{O}_{11} \mathrm{~S}_{2}$

$884.25 \mathrm{~g} / \mathrm{mol}$

Following SOP 8 the synthesis was achieved by starting with 8 . The product 27 was obtained as a yellow solid. 
MS (ESI, MeOH): m/z = 885.3 [M+H $]^{+}, 907.2[\mathrm{M}+\mathrm{Na}]^{+}$.

HR-MS (ESI, MeOH):

$\begin{array}{lll}\mathrm{C}_{43} \mathrm{H}_{45} \mathrm{~N}_{6} \mathrm{O}_{11} \mathrm{~S}[\mathrm{M}+\mathrm{H}]^{+} & \text {calc.: 885.2582 } & \text { mesur.: 885.2572; } \\ \mathrm{C}_{43} \mathrm{H}_{44} \mathrm{~N}_{6} \mathrm{O}_{11} \mathrm{SNa}[\mathrm{M}+\mathrm{Na}]^{+} & \text {calc.: } 907.2402 & \text { mesur.: 907.2385. }\end{array}$

(Z/E)-3-((3-(1H-Benzoimidazol-2-yl)-7-(bis(2-methoxy-2-oxoethyl)amino)-6-(2(2-(bis(2-methoxy-2-oxoethyl)amino)phenoxy)ethoxy)-2H-chromen-2ylidene)amino)propanoic acid

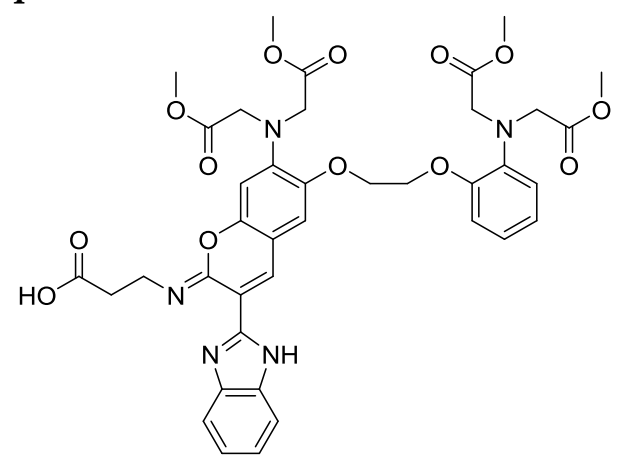

30

$\mathrm{C}_{39} \mathrm{H}_{41} \mathrm{~N}_{5} \mathrm{O}_{13}$ $787.27 \mathrm{~g} / \mathrm{mol}$

Following SOP 8 the synthesis was achieved by starting with $\mathbf{8}$. The product $\mathbf{3 0}$ was obtained as a yellow solid.

MS (ESI, MeOH): m/z = $788.3[\mathrm{M}+\mathrm{H}]^{+}$.

HR-MS (ESI, MeOH):

$\begin{array}{lll}\mathrm{C}_{39} \mathrm{H}_{42} \mathrm{~N}_{5} \mathrm{O}_{13}[\mathrm{M}+\mathrm{H}]^{+} & \text {calc.: 788.2774 } & \text { mesur.: 788.2765; } \\ \mathrm{C}_{39} \mathrm{H}_{41} \mathrm{~N}_{5} \mathrm{O}_{13} \mathrm{Na}[\mathrm{M}+\mathrm{Na}]^{+} & \text {calc.: 810.2593 } & \text { mesur.: 810.2584. }\end{array}$




\subsubsection{Acid-labile BAPTA-based $\mathrm{Ca}^{2+}$-sensor}

\section{Di-tert-butyl 2,2'-((5-(benzyloxy)-2-(2-(2-(bis(2-(tert-butoxy)-2-} oxoethyl)amino)phenoxy)ethoxy)phenyl)azanediyl)diacetate

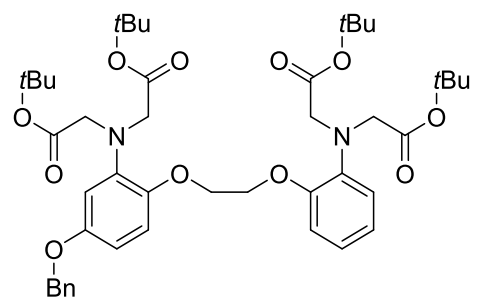

35

$\mathrm{C}_{45} \mathrm{H}_{62} \mathrm{~N}_{2} \mathrm{O}_{11}$

$806.44 \mathrm{~g} / \mathrm{mol}$

2-[2'-(2"'-Aminophenoxy)ethoxy]-5-(benzyloxy)aniline $\quad(5.55 \mathrm{~g}, 15.84 \mathrm{mmol}, 1.00 \mathrm{eq})$, sodium iodide $(6.53 \mathrm{~g}, 43.56 \mathrm{mmol}, 2.75 \mathrm{eq})$ and 1,8-bis(dimethylamino)naphthalene (19.35 g, $90.28 \mathrm{mmol}, 5.70 \mathrm{eq})$ were dissolved in dry ACN (32 mL) under argon atmosphere. tert-Butyl bromoacetate $(21.10 \mathrm{~mL}, 142.70 \mathrm{mmol}, 9.01 \mathrm{eq})$ was slowly added and the solution was stirred at $80{ }^{\circ} \mathrm{C}$ for $24 \mathrm{~h}$. The reaction was quenched with saturated $\mathrm{NaHCO}_{3}$ solution and the crude product was extracted with DCM $(3 \times 100 \mathrm{~mL})$. The organic phase was washed with acetic acid/brine and dried over $\mathrm{MgSO}_{4}$. The solvent was evaporated under reduced pressure, the crude product was purified by flash column chromatography (toluene/EE, 9:1) and obtained as brown solid or yellow oil (8.49 g, $10.52 \mathrm{mmol}, 66 . \%)$.

${ }^{\mathbf{1}} \mathbf{H}-\mathrm{NMR}\left(300 \mathrm{MHz},\left[\mathrm{D}_{6}\right] \mathrm{DMSO}\right): \delta(\mathrm{ppm})=1.29-1.37(\mathrm{~m}, 32 \mathrm{H},-\mathrm{COO} \underline{\mathrm{Bu}}), 3.98-4.07$

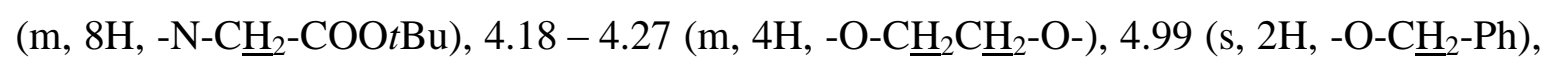
$6.42-6.51\left(\mathrm{~m}, 2 \mathrm{H}, \underline{\mathrm{Ph}}-\mathrm{N}-\left(\mathrm{CH}_{2}\right)_{2^{-}}\right), 6.77-6.90\left(\mathrm{~m}, 4 \mathrm{H}, \underline{\mathrm{Ph}}-\mathrm{N}-\left(\mathrm{CH}_{2}\right)_{2}-\right), 6.92-6.98(\mathrm{~m}$, $\left.1 \mathrm{H}, \underline{\mathrm{Ph}}-\mathrm{N}-\left(\mathrm{CH}_{2}\right)_{2}-\right), 7.26-7.42\left(\mathrm{~m}, 5 \mathrm{H}, \mathrm{O}-\mathrm{CH}_{2}-\underline{\mathrm{Ph}}\right)$.

${ }^{13}$ C-NMR (75 MHz, [D $]$ DMSO): $\delta(\mathrm{ppm})=27.64,54.06,67.47,68.47,69.47,80.15$, $80.25,106.05,106.80,114.62,116.67,119.03,121.09$, 121.36, 127.16, 127.45, 128.13, $137.19,138.79,140.17,143.96,149.85,153.17,169.33,169.40$.

MS (ESI, MeOH): m/z = 807.4 $[\mathrm{M}+\mathrm{H}]^{+}, 829.4[\mathrm{M}+\mathrm{Na}]^{+}$. 
HR-MS (ESI, MeOH):

$\begin{array}{lll}\mathrm{C}_{45} \mathrm{H}_{63} \mathrm{~N}_{2} \mathrm{O}_{11}[\mathrm{M}+\mathrm{H}]^{+} & \text {calc.: 807.4426 } & \text { mesur.: 807.4413; } \\ \mathrm{C}_{45} \mathrm{H}_{62} \mathrm{~N}_{2} \mathrm{O}_{11} \mathrm{Na}[\mathrm{M}+\mathrm{Na}]^{+} & \text {calc.: 829.4246 } & \text { mesur.: 829.4233. }\end{array}$

Di-tert-butyl 2,2'-((5-(benzyloxy)-2-(2-(2-(bis(2-(tert-butoxy)-2oxoethyl)amino)phenoxy)ethoxy)-4-formylphenyl)azanediyl)diacetate

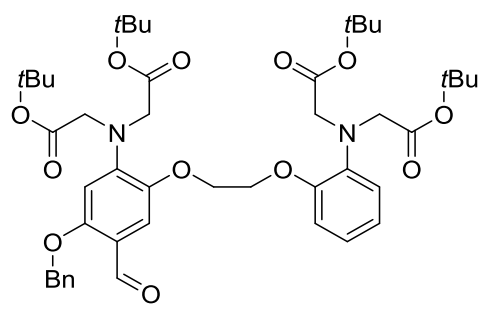

36

$\mathrm{C}_{46} \mathrm{H}_{62} \mathrm{~N}_{2} \mathrm{O}_{12}$

$834.43 \mathrm{~g} / \mathrm{mol}$

Di-tert-butyl 2,2'-((5-(benzyloxy)-2-(2-(2-(bis(2-(tert-butoxy)-2-oxoethyl)amino)phenoxy)ethoxy)phenyl)azanediyl)diacetate ( $8.49 \mathrm{~g}, 10.52 \mathrm{mmol}, 1.00 \mathrm{eq})$ was dissolved in dry DMF $(52 \mathrm{~mL})$ under an argon atmosphere. Pyridine $(8.49 \mathrm{~mL}, 105.19 \mathrm{mmol}$, 10.00 eq) was added and the reaction mixture was cooled to $0{ }^{\circ} \mathrm{C}$. Phosphoryl chloride (7.87 mL, $84.15 \mathrm{mmol}, 8.00 \mathrm{eq}$ ) was added and stirred at $0{ }^{\circ} \mathrm{C}$ for $1.5 \mathrm{~h}$. The reaction was quenched with cold $1 \mathrm{M} \mathrm{KOH}$ solution and the crude product was extracted with DCM $(3 \times 100 \mathrm{~mL})$. Subsequently, the organic phase was washed with brine and dried over $\mathrm{MgSO}_{4}$. The solvent was evaporated under reduced pressure and the crude product was purified by filtration over $\mathrm{SiO}_{2}$ with $\mathrm{EE}$. The contaminated product was obtained as orange solid (8.42 g) and used directly in the next step.

${ }^{1}$ H-NMR $\left(300 \mathrm{MHz},\left[\mathrm{D}_{6}\right] \mathrm{DMSO}\right): \delta(\mathrm{ppm})=1.32 \quad(\mathrm{~s}, \quad 18 \mathrm{H}, \quad-\mathrm{COO} \underline{\mathrm{tBu}}), 1.36 \quad(\mathrm{~s}$, $18 \mathrm{H},-\mathrm{COO} \underline{\mathrm{Bu}}), 4.00\left(\mathrm{~s}, 4 \mathrm{H},-\mathrm{N}-\underline{\mathrm{H}}_{2}-\mathrm{COO} t \mathrm{Bu}\right), 4.20\left(\mathrm{~s}, 4 \mathrm{H},-\mathrm{N}-\underline{\mathrm{C}}_{2}-\mathrm{COO} t \mathrm{Bu}\right), 4.25$ (s, $\left.4 \mathrm{H},-\mathrm{O}-\underline{\mathrm{C}}_{2} \mathrm{C}_{2}{ }_{2}-\mathrm{O}-\right), 5.16$ (s, 2H, -O-C $\left.\underline{\mathrm{H}}_{2}-\mathrm{Ph}\right), 6.42$ (s, 1H, $\left.\underline{\mathrm{Ph}}-\mathrm{N}-\left(\mathrm{CH}_{2}\right)_{2}-\right), 6.77-6.89$ (m, $\left.3 \mathrm{H}, \underline{\mathrm{Ph}}-\mathrm{N}-\left(\mathrm{CH}_{2}\right)_{2}-\right), 6.93-6.99\left(\mathrm{~m}, 1 \mathrm{H}, \underline{\mathrm{Ph}}-\mathrm{N}-\left(\mathrm{CH}_{2}\right)_{2^{-}}\right), 7.20$ (s, 1H, $\left.\underline{\mathrm{Ph}}-\mathrm{N}-\left(\mathrm{CH}_{2}\right)_{2^{-}}\right), 7.32-$ 7.48 (m, overlay by pyridine, $\mathrm{O}-\mathrm{CH}_{2}-\underline{\mathrm{Ph}}$ ), 10.15 (s, $1 \mathrm{H},-\mathrm{C} \underline{\mathrm{HO}}$ ).

${ }^{13}$ C-NMR $\left(75 \mathrm{MHz},\left[\mathrm{D}_{6}\right] \mathrm{DMSO}\right): \delta(\mathrm{ppm})=28.14,54.48,55.09,66.81,67.65,68.54$, $70.97,80.75,81.29,102.78,111.99,115.19,116.82,119.59,121.86,121.96,127.95$, 128.50, 129.02, 136.95, 143.49, 150.34, 169.49, 170.12, 186.44 .

MS (ESI, MeOH): m/z = 835.4 [M+H $]^{+}, 857.4[\mathrm{M}+\mathrm{Na}]^{+}$. 
HR-MS (ESI, MeOH):

$$
\begin{array}{lll}
\mathrm{C}_{46} \mathrm{H}_{63} \mathrm{~N}_{2} \mathrm{O}_{12}[\mathrm{M}+\mathrm{H}]^{+} & \text {calc.: 835.4376 } & \text { mesur.: 835.4364; } \\
\mathrm{C}_{46} \mathrm{H}_{62} \mathrm{~N}_{2} \mathrm{O}_{12} \mathrm{Na}[\mathrm{M}+\mathrm{Na}]^{+} & \text {calc.: 857.4195 } & \text { mesur.: 857.4184 }
\end{array}
$$

Di-tert-butyl 2,2'-((2-(2-(2-(bis(2-(tert-butoxy)-2-oxoethyl)amino)-5-formyl-4hydroxyphenoxy)ethoxy)phenyl)azanediyl)diacetate

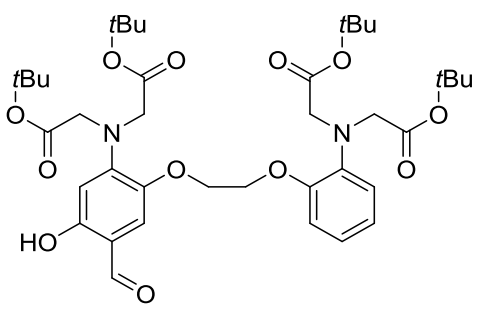

Di-tert-butyl 2,2'-((5-(benzyloxy)-2-(2-(2-(bis(2-(tert-butoxy)-2-oxoethyl)amino)phenoxy)ethoxy)-4-formylphenyl)azanediyl)diacetate (8.42 g) was dissolved in acetic acid $(15.00 \mathrm{~mL} / \mathrm{g}$ educt) and $10 \% \mathrm{Pd} / \mathrm{C}(294.10 \mathrm{mg} / \mathrm{g}$ educt $)$ was added. The solution was stirred for up to $3 \mathrm{~h}$ under $\mathrm{H}_{2}$ atmosphere (room pressure). The reaction solution was filtered over celite and the solvent was evaporated under reduced pressure. The crude product was purified by flash column chromatography (toluene/EE, 9:1 $\rightarrow 8: 2$ ). The product was obtained after lyophilisation as yellow solid (1.61 g, $2.16 \mathrm{mmol}, 21 \%$ over 2 steps).

${ }^{\mathbf{1}} \mathbf{H}-\mathbf{N M R} \quad\left(300 \mathrm{MHz}, \quad\left[\mathrm{D}_{6}\right] \mathrm{DMSO}\right): \delta \quad(\mathrm{ppm})=1.37 \quad(\mathrm{~s}, \quad 18 \mathrm{H}, \quad-\mathrm{COO} \underline{\mathrm{Bu}}), 1.42 \quad(\mathrm{~s}$, $18 \mathrm{H},-\mathrm{COO} t \underline{\mathrm{Bu}}), 4.06\left(\mathrm{~s}, 4 \mathrm{H},,-\mathrm{N}-\mathrm{C} \underline{H}_{2}-\mathrm{COO} t \mathrm{Bu}\right), 4.20\left(\mathrm{~s}, 4 \mathrm{H},-\mathrm{N}-\mathrm{C} \underline{\mathrm{H}}_{2}-\mathrm{COO} t \mathrm{Bu}\right), 4.24-$ $4.33\left(\mathrm{~m}, 4 \mathrm{H},-\mathrm{O}-\mathrm{C}_{2} \mathrm{C}_{2}-\mathrm{O}-\right), 6.16\left(\mathrm{~s}, 1 \mathrm{H}, \underline{\mathrm{Ph}}-\mathrm{N}-\left(\mathrm{CH}_{2}\right)_{2^{-}}\right), 6.83-6.95(\mathrm{~m}, 3 \mathrm{H}$, $\left.\underline{\mathrm{Ph}}-\mathrm{N}-\left(\mathrm{CH}_{2}\right)_{2}{ }^{-}\right), 6.98-7.04\left(\mathrm{~m}, 1 \mathrm{H}, \underline{\mathrm{Ph}}-\mathrm{N}-\left(\mathrm{CH}_{2}\right)_{2^{-}}\right), 7.23$ (s, 1H, $\left.\underline{\mathrm{Ph}}-\mathrm{N}-\left(\mathrm{CH}_{2}\right)_{2}{ }^{-}\right), 9.93$ (s,

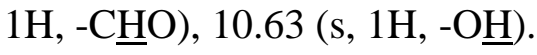

${ }^{13}$ C-NMR (75 MHz, [D 6 DMSO): $\delta(\mathrm{ppm})=27.64,54.00,54.68,67.16,68.24,80.25$, $80.78,103.51,113.48,114.00,114.73,119.18,121.34,121.49,139.00,142.06,147.12$, $149.88,157.42,168.89,169.60,189.67$.

MS (ESI, MeOH): m/z = 745.4 $[\mathrm{M}+\mathrm{H}]^{+}, 767.4[\mathrm{M}+\mathrm{Na}]^{+}$. 
HR-MS (ESI, MeOH):

$\begin{array}{lll}\mathrm{C}_{45} \mathrm{H}_{63} \mathrm{~N}_{2} \mathrm{O}_{11}[\mathrm{M}+\mathrm{H}]^{+} & \text {calc.: 745.3906 } & \text { mesur.: 745.3901; } \\ \mathrm{C}_{45} \mathrm{H}_{62} \mathrm{~N}_{2} \mathrm{O}_{11} \mathrm{Na}[\mathrm{M}+\mathrm{Na}]^{+} & \text {calc.: 767.3725 } & \text { mesur.: 767.3721. }\end{array}$

Di-tert-butyl 2,2'-((3-(benzothiazol-2-yl)-6-(2-(2-(bis(2-(tert-butoxy)-2oxoethyl)amino)phenoxy)ethoxy)-2-imino-2H-chromen-7-yl)azanediyl)diacetate

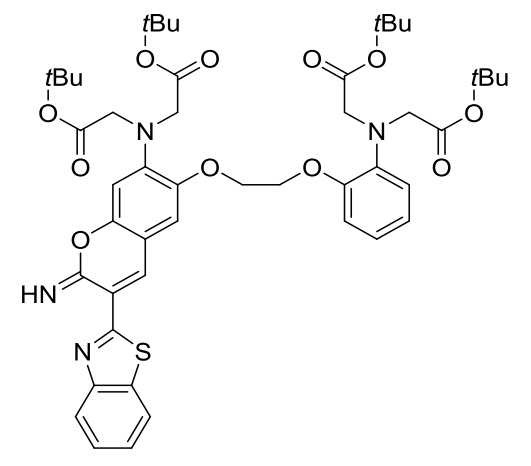

6

$\mathrm{C}_{48} \mathrm{H}_{60} \mathrm{~N}_{4} \mathrm{O}_{11} \mathrm{~S}$

$900.40 \mathrm{~g} / \mathrm{mol}$

Piperidine (4.51 mL, $45.64 \mathrm{mmol}, 10.00 \mathrm{eq}$ ) was added to a solution of di-tert-butyl 2,2'((2-(2-(2-(bis(2-(tert-butoxy)-2-oxoethyl)amino)-5-formyl-4-

hydroxyphenoxy)ethoxy)phenyl)azanediyl)diacetate $(3.40 \mathrm{~g}, 4.56 \mathrm{mmol}, 1.00 \mathrm{eq})$ in dry $\mathrm{MeOH}(10 \mathrm{~mL})$. Then benzothiazole-2-yl-acetonitrile (795 mg, $4.56 \mathrm{mmol}, 1.00 \mathrm{eq}$ ) was added and the solution was stirred overnight under argon atmosphere at room temperature. The precipitate was filtered and washed with dry ether and dried under reduced pressure. The product was obtained as yellow solid (3.03 g, $3.37 \mathrm{mmol}, 74 \%)$

${ }^{\mathbf{1}} \mathbf{H}-\mathbf{N M R}\left(300 \mathrm{MHz}, \mathrm{CDCl}_{3}\right): \delta(\mathrm{ppm})=1.42(\mathrm{~s}, 18 \mathrm{H},-\mathrm{COO} \underline{t \mathrm{Bu}}), 1.48\left(\mathrm{~s}, 18 \mathrm{H},-\mathrm{COO}^{\mathrm{t}} \underline{\mathrm{Bu}}\right)$, $4.09\left(\mathrm{~s}, \quad 4 \mathrm{H}, \quad-\mathrm{N}-\mathrm{C}_{2}-\mathrm{COO}{ }^{t} \mathrm{Bu}\right), 4.17\left(\mathrm{~s}, \quad 4 \mathrm{H}, \quad-\mathrm{N}_{-}-\underline{\mathrm{H}}_{2}-\mathrm{COO}{ }^{\mathrm{B}} \mathrm{Bu}\right), 4.34-4.40 \quad(\mathrm{~m}$, $\left.4 \mathrm{H},-\mathrm{O}-\underline{\mathrm{C}}_{2} \mathrm{C}_{2_{2}}-\mathrm{O}-\right), 6.56$ (s, 1H, $\left.\underline{\mathrm{Ph}}-\mathrm{N}-\left(\mathrm{CH}_{2}\right)_{2}-\right), 6.90-6.95$ (m, 4H, $\left.\underline{\mathrm{Ph}}-\mathrm{N}-\left(\mathrm{CH}_{2}\right)_{2}-\right), 7.06$ $\left(\mathrm{s}, 1 \mathrm{H}, \underline{\mathrm{Ph}}-\mathrm{N}-\left(\mathrm{CH}_{2}\right)_{2}-\right), 7.33-7.56(\mathrm{~m}, 2 \mathrm{H}, \underline{\text { benzothiazole }}), 7.93\left(\mathrm{~d},{ }^{3} J_{\mathrm{H}, \mathrm{H}}=7.8 \mathrm{~Hz}, 1 \mathrm{H}\right.$, benzothiazole $), 8.05\left(\mathrm{~d},{ }^{3} J_{\mathrm{H}, \mathrm{H}}=8.0 \mathrm{~Hz}, 1 \mathrm{H}\right.$, benzothiazole $), 8.48\left(\mathrm{bs}, 1 \mathrm{H}, \mathrm{H}_{\mathrm{Ar}}\right)$.

${ }^{13}$ C-NMR $\left(75 \mathrm{MHz}, \mathrm{CDCl}_{3}\right): \delta(\mathrm{ppm})=28.14,54.54,54.85,67.59,68.69,81.14,81.91$, 104.24, 113.39, 115.59, 119.91, 121.33, 122.16, 122.26, 126.17, 139.89, 146.18, 149.86, $150.34,169.52,170.33$.

MS (ESI, MeOH): m/z = $901.4[\mathrm{M}+\mathrm{H}]^{+}, 1802.9[2 \mathrm{M}+\mathrm{H}]^{+}$. 
HR-MS (ESI, MeOH):

$\begin{array}{lll}\mathrm{C}_{48} \mathrm{H}_{61} \mathrm{~N}_{4} \mathrm{O}_{11} \mathrm{~S}[\mathrm{M}+\mathrm{H}]^{+} & \text {calc.: } 901.4052 & \text { mesur.: 901.4051, } \\ \mathrm{C}_{48} \mathrm{H}_{60} \mathrm{~N}_{4} \mathrm{O}_{11} \mathrm{SNa}[\mathrm{M}+\mathrm{Na}]^{+} & \text {calc.: } 923.3872 & \text { mesur.: 923.3873. }\end{array}$

(Z/E)-3-((3-(Benzothiazol-2-yl)-7-(bis(2-(tert-butoxy)-2-oxoethyl)amino)-6-(2(2-(bis(2-(tert-butoxy)-2-oxoethyl)amino)phenoxy)ethoxy)-2H-chromen-2ylidene)amino)propanoic acid

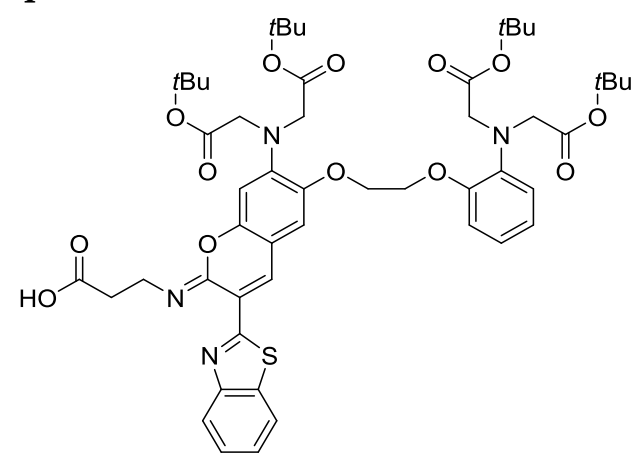

38

$\mathrm{C}_{51} \mathrm{H}_{64} \mathrm{~N}_{4} \mathrm{O}_{13} \mathrm{~S}$

$972.42 \mathrm{~g} / \mathrm{mol}$

Following SOP 838 was achieved by using di-tert-butyl 2,2'-((3-(benzothiazol-2-yl)-6-(2(2-(bis(2-(tert-butoxy)-2-oxoethyl)amino)phenoxy)ethoxy)-2-imino-2H-chromen-7-yl)azanediyl)diacetate $(2.55 \mathrm{~g}, 2.83 \mathrm{mmol}, 1.00 \mathrm{eq})$ and 3-aminopropanoic acid hydrochloride (250 mg, $2.83 \mathrm{mmol}, 1.00 \mathrm{eq})$. The crude product was purified by flash column chromatography (pentane/EE, $1: 2 \rightarrow 0: 1$ ). The product was obtained after lyophilisation as orange solid (1.49 g, $1.53 \mathrm{mmol}, 54 \%)$.

${ }^{\mathbf{1}} \mathbf{H}-\mathbf{N M R}\left(300 \mathrm{MHz}, \mathrm{CDCl}_{3}\right): \delta(\mathrm{ppm})=1.38(\mathrm{~s}, 18 \mathrm{H},-\mathrm{COO} \underline{\underline{\mathrm{Bu}}}), 1.44\left(\mathrm{~s}, 18 \mathrm{H},-\mathrm{COO}^{\mathrm{t}} \underline{\mathrm{Bu}}\right)$, $2.79-2.86\left(\mathrm{~m}, 2 \mathrm{H}, \mathrm{N}-\mathrm{C}_{2}-\mathrm{CH}_{2}-\mathrm{COOH}\right.$ ), $3.82-3.89$ (m, 2H, N-CH${ }_{2}-\underline{\mathrm{C}}_{2}-\mathrm{COOH}$ ), 4.06 $\left(\mathrm{s}, 4 \mathrm{H},-\mathrm{N}-\underline{\mathrm{C}}_{2}-\mathrm{COO}^{\mathrm{t}} \mathrm{Bu}\right), 4.15\left(\mathrm{~s}, 4 \mathrm{H},-\mathrm{N}_{-} \underline{\mathrm{H}}_{2}-\mathrm{COO}{ }^{\mathrm{B}} \mathrm{Bu}\right), 4.32-4.38(\mathrm{~m}, 4 \mathrm{H},-\mathrm{O}-$ $\left.\mathrm{CH}_{2} \mathrm{CH}_{2}-\mathrm{O}-\right), 6.56$ (s, 1H, $\left.\underline{\mathrm{Ph}}-\mathrm{N}-\left(\mathrm{CH}_{2}\right)_{2}-\right), 6.88-6.91$ (m, 4H, $\left.\underline{\mathrm{Ph}}-\mathrm{N}-\left(\mathrm{CH}_{2}\right)_{2}-\right), 7.05$ (s, 1H, Ph-N-( $\left.\left.\mathrm{CH}_{2}\right)_{2}\right)^{-}, 7.25-7.44(\mathrm{~m}, 2 \mathrm{H}$, benzothiazole $), 7.81-7.87(\mathrm{~m}, 1 \mathrm{H}$, benzothiazole $)$, $7.94-7.99(\mathrm{~m}, 1 \mathrm{H}$, benzothiazole $), 8.48\left(\mathrm{~s}, 1 \mathrm{H}, \mathrm{H}_{\mathrm{Ar}}\right)$.

${ }^{13}$ C-NMR $\left(126 \mathrm{MHz}, \mathrm{CDCl}_{3}\right): \delta(\mathrm{ppm})=28.17,28.19$, 35.26, 41.90, 54.57, 54.88, 67.67, $68.65,81.11,81.88,104.34,111.77,113.40,115.64,118.83,119.88,121.30,122.10$, $122.19,122.54,124.65,125.92$, 134.88, 136.38, 139.80, 143.97, 146.44, 149.26, 150.11, $150.26,152.18,161.23,169.41,170.17,175.52$. 
MS (ESI, MeOH): m/z = $973.5[\mathrm{M}+\mathrm{H}]^{+}, 995.5[\mathrm{M}+\mathrm{Na}]^{+}$.

HR-MS (ESI, MeOH):

$\begin{array}{lll}\mathrm{C}_{51} \mathrm{H}_{65} \mathrm{~N}_{4} \mathrm{O}_{13} \mathrm{~S}[\mathrm{M}+\mathrm{H}]^{+} & \text {calc.: } 973.4263 & \text { mesur.: 973.4265; } \\ \mathrm{C}_{51} \mathrm{H}_{64} \mathrm{~N}_{4} \mathrm{O}_{13} \mathrm{SNa}[\mathrm{M}+\mathrm{Na}]^{+} & \text {calc.: } 995.4083 & \text { mesur.: 995.4083. }\end{array}$

\subsubsection{Acid-labile MOBHA-based $\mathrm{Ca}^{2+}$-sensor}

2-(4-(Benzyloxy)-2-nitrophenoxy)methyl acetate<smiles>COC(=O)COc1ccc(OCc2ccccc2)cc1[N+](=O)[O-]</smiles>

39

$\mathrm{C}_{16} \mathrm{H}_{15} \mathrm{NO}_{6}$

$317.09 \mathrm{~g} / \mathrm{mol}$

4-Benzyloxy-2-nitrophenol (6.00 g, $24.50 \mathrm{mmol}, 1.00 \mathrm{eq})$ and dry potassium carbonate (3.38 g, $24.50 \mathrm{mmol}, 1.00 \mathrm{eq})$ were dissolved in dry DMF (14 mL) and heated to $120{ }^{\circ} \mathrm{C}$ under an argon atmosphere. Methyl bromoacetate $(2.55 \mathrm{~mL}, 26.90 \mathrm{mmol}, 1.10 \mathrm{eq})$ was added and the mixture was stirred for 5-10 min. The reaction process was monitored by TLC. Afterwards, the mixture was diluted with saturated sodium bicarbonate solution, extracted with DCM and dried over $\mathrm{MgSO}_{4}$. The solvent was evaporated under reduced pressure and the crude product was purified by flash column chromatography (pentane/EE, 3:1). The product was obtained as yellow solid (6.40 g, $20.20 \mathrm{mmol}, 82 \%)$.

TLC (pentane/EE 2:1): $R_{f}=0.63$.

${ }^{1}$ H-NMR (300 MHz, [D 6 DDMSO): $\delta(\mathrm{ppm})=3.69$ (s, 3H, -COOMe $), 4.95$ (s, 2H, -O-C $\underline{\mathrm{H}}_{2}-$ COOMe), 5.15 (s, 2H, -O-C $\left.\underline{\mathrm{H}}_{2}-\mathrm{Ph}\right), 7.22-7.49$ (m, 7H, O- $\left.\mathrm{CH}_{2}-\underline{\mathrm{Ph}}, \underline{\mathrm{Ph}}-\mathrm{NO}_{2}\right), 7.52-7.60$ $\left(\mathrm{m}, 1 \mathrm{H}, \underline{\mathrm{Ph}}-\mathrm{NO}_{2}\right)$.

${ }^{13}$ C-NMR (75 MHz, $\left.\left[\mathrm{D}_{6}\right] \mathrm{DMSO}\right): \delta(\mathrm{ppm})=52.39,66.56,70.63,111.03,117.39,121.35$, $128.27,128.48,128.93,136.89,140.49,144.82,152.55,169.08$.

MS (ESI, MeOH): m/z = $318.1[\mathrm{M}+\mathrm{H}]^{+}, 340.1[\mathrm{M}+\mathrm{Na}]^{+}, 657.2[2 \mathrm{M}+\mathrm{Na}]^{+}$. 
HR-MS (ESI, MeOH):

$\begin{array}{lll}\mathrm{C}_{16} \mathrm{H}_{16} \mathrm{NO}_{6}[\mathrm{M}+\mathrm{H}]^{+} & \text {calc.: } 318.0972 & \text { mesur.: 318.0967; } \\ \mathrm{C}_{16} \mathrm{H}_{15} \mathrm{NO}_{6} \mathrm{Na}[\mathrm{M}+\mathrm{Na}]^{+} & \text {calc.: } 340.0792 & \text { mesur.: 340.0793. }\end{array}$

\section{6-(Benzyloxy)-2H-benzo[b][1,4]oxazin-3(4H)-one}<smiles>O=C1COc2ccc(OCc3ccccc3)cc2N1</smiles>

40

$\mathrm{C}_{15} \mathrm{H}_{13} \mathrm{NO}_{3}$

$255.09 \mathrm{~g} / \mathrm{mol}$

2-(4-(Benzyloxy)-2-nitrophenoxy)methyl acetate $(5.41 \mathrm{~g}, 17.10 \mathrm{mmol}, 1.00 \mathrm{eq})$ and ammonium chloride (5.63 g, $105.20 \mathrm{mmol}, 6.20 \mathrm{eq})$ were dissolved in acetone $/ \mathrm{H}_{2} \mathrm{O}(3: 1)$ under argon atmosphere in a miniclave (BÜCHI, Uster, Switzerland). Iron powder (7.33 g, $131.30 \mathrm{mmol}, 7.70 \mathrm{eq}$ ) was added and the mixture was stirred for $1 \mathrm{~h}$ at $80^{\circ} \mathrm{C}$. The crude product was purified by filtration over $\mathrm{SiO}_{2}$ in DCM. The solvent was dried over $\mathrm{MgSO}_{4}$, evaporated under reduced pressure and the product was obtained as colourless solid $(4.30 \mathrm{~g}, 16.70 \mathrm{mmol}, 98 \%)$.

TLC (pentane/EE 2:1): $R_{f}=0.40$.

${ }^{\mathbf{1}} \mathbf{H}-\mathrm{NMR}\left(300 \mathrm{MHz},\left[\mathrm{D}_{6}\right] \mathrm{DMSO}\right): \delta(\mathrm{ppm})=4.49$ (s, 2H, CO-C $\left.\underline{\mathrm{H}}_{2}-\mathrm{O}\right), 5.02(\mathrm{~s}, 2 \mathrm{H},-\mathrm{O}-$ $\left.\mathrm{C}_{2}-\mathrm{Ph}\right), 6.52-6.62$ (m, 2H, - $\left.\underline{\mathrm{Ph}}-\mathrm{NH}-\right), 6.81-6.92$ (m, 1H, - $\left.\underline{\mathrm{Ph}}-\mathrm{NH}-\right), 7.26-7.48$ (m, 5H,

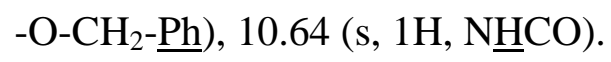

${ }^{13}$ C-NMR (75 MHz, [D 6 DMSO): $\delta(\mathrm{ppm})=67.31,70.11,103.33,108.91,116.97,128.05$, $128.25,128.52,128.86,137.50,137.83,154.09,165.69$.

MS (ESI, MeOH): m/z = 256.1 $[\mathrm{M}+\mathrm{H}]^{+}, 278.1[\mathrm{M}+\mathrm{Na}]^{+}, 533.2[2 \mathrm{M}+\mathrm{Na}]^{+}$.

HR-MS (ESI, MeOH):
$\mathrm{C}_{15} \mathrm{H}_{14} \mathrm{NO}_{3}[\mathrm{M}+\mathrm{H}]^{+}$
calc.: 256.0968
mesur.: 256.0977;
$\mathrm{C}_{15} \mathrm{H}_{13} \mathrm{NO}_{3} \mathrm{Na}[\mathrm{M}+\mathrm{Na}]^{+}$
calc.: 278.0788
mesur.: 278.0797. 


\section{tert-Butyl 6-(benzyloxy)-3-oxo-2,3-dihydro-4H-benzo[b][1,4]oxazine-4- carboxylate}

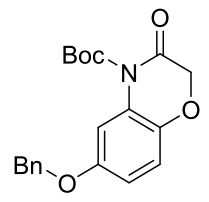

41

$\mathrm{C}_{20} \mathrm{H}_{21} \mathrm{NO}_{5}$

$355.14 \mathrm{~g} / \mathrm{mol}$

6-(Benzyloxy)-2H-benzo[b][1,4] oxazin-3(4H)-one $\quad(4.26 \mathrm{~g}, \quad 16.69 \mathrm{mmol}, \quad 1.0 \mathrm{eq}) \quad$ was dissolved in dry THF (100 mL). Di-tert-butyl dicarbonate $(5.11 \mathrm{~g}, 23.41 \mathrm{mmol}, 1.5 \mathrm{eq})$, diisopropylethylamine $(5.31 \mathrm{~mL}, \quad 31.21 \mathrm{mmol}, 2 \mathrm{eq})$ and 4-(dimethylamino)-pyridin (catalytic amount) were added and the solution was stirred at RT for $5 \mathrm{~h}$. The reaction process was monitored by TLC. Subsequently, the reaction was treated with a $3 \mathrm{M} \mathrm{KOH}$ solution, the solvent was removed under reduced pressure and the crude product was purified by flash column chromatography (pentane/EE, 2:1). The product was obtained as yellow oil (4.72 g, $13.28 \mathrm{mmol}, 80 \%)$

TLC (pentane/EE 2:1): $R_{f}=0.80$

${ }^{1}$ H-NMR $\left(300 \mathrm{MHz},\left[\mathrm{D}_{6}\right] \mathrm{DMSO}\right): \delta(\mathrm{ppm})=1.52(\mathrm{~s}, 9 \mathrm{H}, \underline{\mathrm{Boc}}-\mathrm{NH}), 4.63\left(\mathrm{~s}, 2 \mathrm{H}, \mathrm{CO}-\underline{\mathrm{H}}_{2}-\right.$ O), 5.07 (s, 2H, -O-C $\left.\underline{H}_{2}-\mathrm{Ph}\right), 6.69\left(\mathrm{~d},{ }^{3} J_{\mathrm{H}, \mathrm{H}}=2.8 \mathrm{~Hz}, 1 \mathrm{H},-\underline{\mathrm{Ph}}-\mathrm{NH}-\right), 6.75-6.82(\mathrm{~m}, 1 \mathrm{H},-$ $\underline{\mathrm{Ph}}-\mathrm{NH}-), 7.00-7.06$ (m, 1H, - $\underline{\mathrm{Ph}}-\mathrm{NH}-), 7.28-7.45$ (m, 5H, -O-CH $2-\underline{\mathrm{Ph}})$.

${ }^{13}$ C-NMR (75 MHz, [D 6 DMSO): $\delta(\mathrm{ppm})=27.69,68.29,70.32,86.17,105.02,111.61$, 118.21, 127.03, 128.00, 128.34, 128.88, 128.93, 137.29, 139.35, 149.65, 154.16, 164.66.

MS (ESI, MeOH): $\mathrm{m} / \mathrm{z}=356.1[\mathrm{M}+\mathrm{H}]^{+}, 378.1[\mathrm{M}+\mathrm{Na}]^{+}, 733.3[2 \mathrm{M}+\mathrm{Na}]^{+}$.

HR-MS (ESI, MeOH):
$\mathrm{C}_{20} \mathrm{H}_{22} \mathrm{NO}_{5}[\mathrm{M}+\mathrm{H}]^{+}$
calc.: 356.1492
mesur.: 356.1506;
$\mathrm{C}_{20} \mathrm{H}_{21} \mathrm{NO}_{5} \mathrm{Na}[\mathrm{M}+\mathrm{Na}]^{+}$
calc.: 378.1312
mesur.: 378.1327. 


\section{tert-Butyl (5-(benzyloxy)-2-(2-morpholino-2-oxoethoxy)phenyl)carba-mate}<smiles>O=C(Cc1ccccc1)Nc1cccc(OCC(=O)OCc2ccccc2)c1</smiles>

42

$\mathrm{C}_{24} \mathrm{H}_{30} \mathrm{~N}_{2} \mathrm{O}_{6}$

$442.21 \mathrm{~g} / \mathrm{mol}$

tert-Butyl 6-(benzyloxy)-3-oxo-2,3-dihydro-4H-benzo[b][1,4]oxazine-4-carboxylate (4.72 g, $13.28 \mathrm{mmol}, 1.00 \mathrm{eq})$ was dissolved in dry THF $(30 \mathrm{~mL})$ and cooled down to $0{ }^{\circ} \mathrm{C}$. Isobutyl chloroformate $(2.07 \mathrm{~g}, 15.17 \mathrm{mmol}, \quad 1.20 \mathrm{eq})$, triethylamine $(2.10 \mathrm{~mL}$, $15.17 \mathrm{mmol}, 1.20 \mathrm{eq})$ and morpholine (2.86 g, $32.87 \mathrm{mmol}, 2.60 \mathrm{eq})$ were added and the solution was stirred at RT for $4-6 \mathrm{~h}$. The reaction was quenched with acetic acid followed by the addition of a saturated aqueous solution of sodium bicarbonate. The aqueous phase was extracted with ethyl acetate $(3 \times 100 \mathrm{~mL})$, the combined organic phases were washed with saturated aqueous solution of ammonium chloride $(3 \times 60 \mathrm{~mL})$ and saturated aqueous solution of sodium chloride $(60 \mathrm{~mL})$, followed by drying over magnesium sulphate. The solvent was removed under reduced pressure. The crude product was purified by flash column chromatography (Pentane/EE 1:2). The product was obtained as white solid (3.13 g, $7.07 \mathrm{mmol}, 53 \%)$.

TLC (pentane/EE 1:2): $R_{f}=0.30$.

${ }^{\mathbf{1}} \mathbf{H}-\mathbf{N M R}\left(300 \mathrm{MHz},\left[\mathrm{D}_{6}\right] \mathrm{DMSO}\right): \delta(\mathrm{ppm})=1.48(\mathrm{~s}, 9 \mathrm{H}, \underline{\text { Boc-NH}}), 3.34-3.48(\mathrm{~m}$, $\left.4 \mathrm{H},-\mathrm{N}-\mathrm{CH}_{2}-\mathrm{CH}_{2}-\mathrm{O}\right), 3.53-3.61$ (m, 4H, -N-CH$\left.{ }_{2}-\underline{\mathrm{C}}_{2}-\mathrm{O}\right), 4.87$ (s, 2H, CON-C $\left.\underline{H}_{2}-\mathrm{O}\right)$, $5.03\left(\mathrm{~s}, 2 \mathrm{H},-\mathrm{O}-\underline{\mathrm{C}}_{2}-\mathrm{Ph}\right), 6.62\left(\mathrm{dd},{ }^{3} J_{\mathrm{H}, \mathrm{H}}=8.9 \mathrm{~Hz}, 3.0 \mathrm{~Hz}, 1 \mathrm{H},-\underline{\mathrm{Ph}}-\mathrm{NH}-\right), 6.98$ (d, $\left.{ }^{3} J_{\mathrm{H}, \mathrm{H}}=8.9 \mathrm{~Hz}, 1 \mathrm{H},-\underline{\mathrm{Ph}}-\mathrm{NH}-\right), 7.27-7.47\left(\mathrm{~m}, 5 \mathrm{H},-\mathrm{O}-\mathrm{CH}_{2}-\underline{\mathrm{Ph}}\right), 7.53\left(\mathrm{~d},{ }^{3} J_{\mathrm{H}, \mathrm{H}}=3.0 \mathrm{~Hz}, 1 \mathrm{H}\right.$, - $\underline{\mathrm{Ph}}-\mathrm{NH}-), 8.59$ (s, 1H, Boc-N $\underline{\mathrm{H}})$.

${ }^{13}$ C-NMR (75 MHz, [D 6 DMSO): $\delta(\mathrm{ppm})=28.50,42.11,44.73,66.29,66.42,69.67$, $69.99,79.92$, 107.33, 108.66, 117.18, 127.99, 128.18, 128.83, 130.85, 137.69, 142.93, $152.83,153.81,167.74$.

MS (ESI, MeOH): m/z = 443.2[M+H] $]^{+}, 465.2[\mathrm{M}+\mathrm{Na}]^{+}, 907.4[2 \mathrm{M}+\mathrm{Na}]^{+}$. 
HR-MS (ESI, MeOH):

$\begin{array}{lll}\mathrm{C}_{24} \mathrm{H}_{31} \mathrm{~N}_{2} \mathrm{O}_{6}[\mathrm{M}+\mathrm{H}]^{+} & \text {calc.: 443.2177 } & \text { mesur.: 443.2187; } \\ \mathrm{C}_{24} \mathrm{H}_{30} \mathrm{~N}_{2} \mathrm{O}_{6} \mathrm{Na}[\mathrm{M}+\mathrm{Na}]^{+} & \text {calc.: 465.1996 } & \text { mesur.: 465.2008; } \\ \mathrm{C}_{24} \mathrm{H}_{34} \mathrm{~N}_{3} \mathrm{O}_{6}\left[\mathrm{M}+\mathrm{NH}_{4}\right]^{+} & \text {calc.: 460.2442 } & \text { mesur.: 460.2453. }\end{array}$

\section{Di-tert-butyl 2,2'-((5-(benzyloxy)-2-(2-morpholino-2- oxoethoxy)phenyl)azanediyl)diacetate}<smiles>CCCCOC(=O)CN(CC(=O)OCc1ccccc1)c1cc(OCc2ccccc2)ccc1OCC(=O)N1CCOCC1</smiles>

43

$\mathrm{C}_{31} \mathrm{H}_{42} \mathrm{~N}_{2} \mathrm{O}_{8}$

$570.29 \mathrm{~g} / \mathrm{mol}$

tert-Butyl (5-(benzyloxy)-2-(2-morpholino-2-oxoethoxy)phenyl)carbamate (3.13 g, $7.07 \mathrm{mmol}, 1.00 \mathrm{eq})$ was suspended in $4 \mathrm{M} \mathrm{aq} \mathrm{HCl}$ in dioxane $(40 \mathrm{~mL})$ and stirred for $2 \mathrm{~h}$. The solvent was evaporated under reduced pressure and the crude product was lyophilised from dioxane. The intermediate product was obtained as white solid $(2.42 \mathrm{~g}, 7.07 \mathrm{mmol})$ and was dissolved in dry ACN (7.8 mL) under argon atmosphere. Sodium iodide $(1.59 \mathrm{~g}$, $10.60 \mathrm{mmol}, 1.50 \mathrm{eq})$, 1,8-bis(dimethylamino)naphthalene (6.06 g, $28.27 \mathrm{mmol}, 4.00 \mathrm{eq})$ and tert-butyl bromoacetate $(5.22 \mathrm{~mL}, 35.34 \mathrm{mmol}, 5.00 \mathrm{eq})$ were added slowly and the solution was stirred at $80{ }^{\circ} \mathrm{C}$ for $24 \mathrm{~h}$. The reaction was quenched with saturated $\mathrm{NaHCO}_{3}$ solution, the crude product was extracted with DCM $(3 \times 100 \mathrm{~mL})$, the organic phase was washed with acetic acid/brine and dried over $\mathrm{MgSO}_{4}$. The solvent was evaporated under reduced pressure, the crude product was purified by flash column chromatography (toluene/EE) and obtained as red oil (2.92 g, $7.07 \mathrm{mmol}, 73 \%$ ).

${ }^{1}$ H-NMR (300 MHz, [D 6 ]DMSO): $\delta(\mathrm{ppm})=1.37$ (s, 18H, -COOt $\left.\underline{\mathrm{Bu}}\right), 3.41-3.48(\mathrm{~m}, 4 \mathrm{H}$, $\left.-\mathrm{N}-\underline{\mathrm{H}}_{2}-\mathrm{CH}_{2}-\mathrm{O}\right), 3.53-3.60\left(\mathrm{~m}, 4 \mathrm{H},-\mathrm{N}-\mathrm{CH}_{2}-\underline{\mathrm{C}}_{2}-\mathrm{O}\right), 4.01-4.07\left(\mathrm{~m}, 4 \mathrm{H},-\mathrm{N}-\mathrm{C}_{2}-\right.$ $\mathrm{COO} t \mathrm{Bu}), 4.72\left(\mathrm{~s}, 2 \mathrm{H}, \mathrm{CON}-\underline{\mathrm{H}}_{2}-\mathrm{O}\right), 4.99$ (s, 2H, -O-C $\left.\underline{\mathrm{H}}_{2}-\mathrm{Ph}\right), 6.36-6.51$ (m, 2H, - $\underline{\mathrm{Ph}}-$ $\mathrm{NH}-), 6.80$ (d, $\left.{ }^{3} J_{\mathrm{H}, \mathrm{H}}=8.8 \mathrm{~Hz}, 1 \mathrm{H},-\underline{\mathrm{Ph}}-\mathrm{NH}-\right), 7.28-7.43$ (m, 5H, -O-CH $\left.-\underline{\mathrm{Ph}}\right)$.

${ }^{13}$ C-NMR (75 MHz, [D 6 DMSO): $\delta(\mathrm{ppm})=28.21,54.53,60.20,66.51,67.67,69.93$, $80.80,106.24$, 107.17, 115.90, 127.87, 128.14, 128.82, 137.87, 140.28, 144.20, 153.62, $166.74,170.24$. 
MS (ESI, MeOH): m/z = $571.3[\mathrm{M}+\mathrm{H}]^{+}, 593.3[\mathrm{M}+\mathrm{Na}]^{+}$.

\section{HR-MS (ESI, MeOH):}

$$
\begin{array}{lll}
\mathrm{C}_{31} \mathrm{H}_{43} \mathrm{~N}_{2} \mathrm{O}_{8}[\mathrm{M}+\mathrm{H}]^{+} & \text {calc.: 571.3014 } & \text { mesur.: 571.3015; } \\
\mathrm{C}_{31} \mathrm{H}_{42} \mathrm{~N}_{2} \mathrm{O}_{8} \mathrm{Na}[\mathrm{M}+\mathrm{Na}]^{+} & \text {calc.: 593.2833 } & \text { mesur.: 593.2832. }
\end{array}
$$

\section{Di-tert-butyl 2,2'-((5-(benzyloxy)-4-formyl-2-(2-morpholino-2- oxoethoxy)phenyl)azanediyl)diacetate}

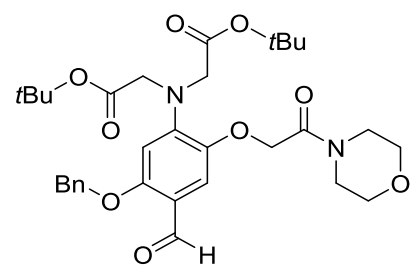

44

$\mathrm{C}_{32} \mathrm{H}_{42} \mathrm{~N}_{2} \mathrm{O}_{9}$

$598.29 \mathrm{~g} / \mathrm{mol}$

Di-tert-butyl 2,2'-((5-(benzyloxy)-2-(2-morpholino-2-oxoethoxy)phenyl)azanediyl)diacetate $(2.92 \mathrm{~g}, 5.12 \mathrm{mmol}, 1.00 \mathrm{eq})$ was dissolved in dry DMF (25.0 mL) under argon atmosphere. Pyridine ( $4.13 \mathrm{~mL}, 51.17 \mathrm{mmol}, 10.00 \mathrm{eq})$ was added and the reaction mixture was cooled to $0{ }^{\circ} \mathrm{C}$. Phosphoryl chloride $(3.83 \mathrm{~mL}, 40.93 \mathrm{mmol}, 8.00 \mathrm{eq})$ was added and stirred at $0{ }^{\circ} \mathrm{C}$ for $1.5 \mathrm{~h}$. The reaction was quenched with cold $1 \mathrm{M} \mathrm{KOH}$ solution, the crude product was extracted with DCM $(3 \times 100 \mathrm{~mL})$, the organic phase was washed with brine and dried over $\mathrm{MgSO}_{4}$. The solvent was evaporated under reduced pressure and the crude product was purified by filtration over $\mathrm{SiO}_{2}$ with $\mathrm{EE}$. The product was obtained as brown solid (2.52 g) and used directly in the next step.

${ }^{\mathbf{1}} \mathbf{H}-\mathbf{N M R}\left(300 \mathrm{MHz},\left[\mathrm{D}_{6}\right] \mathrm{DMSO}\right): \delta(\mathrm{ppm})=1.41(\mathrm{~s}, 18 \mathrm{H},-\mathrm{COO} \underline{\mathrm{Bu}}), 3.43-3.50(\mathrm{~m}, 4 \mathrm{H}$, $-\mathrm{N}-\mathrm{CH}_{2}-\mathrm{CH}_{2}-\mathrm{O}$ ), $3.54-3.63$ (m, overlaid by dioxane, $\left.-\mathrm{N}-\mathrm{CH}_{2}-\mathrm{C}_{2}-\mathrm{O}\right), 4.22$ (s, 4H, -N-

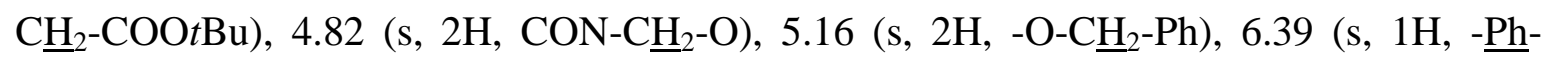

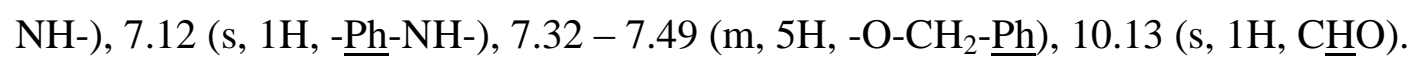

${ }^{13}$ C-NMR (75 MHz, [D 6 DMSO): $\delta(p p m)=28.23,42.09,45.09,55.13,66.74,66.81$, $70.96,81.16,102.58,111.31,116.64,127.95,128.50,129.02,136.96,143.15,146.89$, $157.54,166.22,169.72,186.45$.

MS (ESI, MeOH): m/z = 599.3 [M+H] $]^{+}, 621.3[\mathrm{M}+\mathrm{Na}]^{+}$. 
HR-MS (ESI, MeOH):

$\begin{array}{lll}\mathrm{C}_{32} \mathrm{H}_{43} \mathrm{~N}_{2} \mathrm{O}_{9}[\mathrm{M}+\mathrm{H}]^{+} & \text {calc.: 599.2963 } & \text { mesur.: 599.2965; } \\ \mathrm{C}_{32} \mathrm{H}_{42} \mathrm{~N}_{2} \mathrm{O}_{9} \mathrm{Na}[\mathrm{M}+\mathrm{Na}]^{+} & \text {calc.: 621.2783 } & \text { mesur.: 621.2782. }\end{array}$

\section{Di-tert-butyl 2,2'-((4-formyl-5-hydroxy-2-(2-morpholino-2- oxoethoxy)phenyl)azanediyl)diacetate}

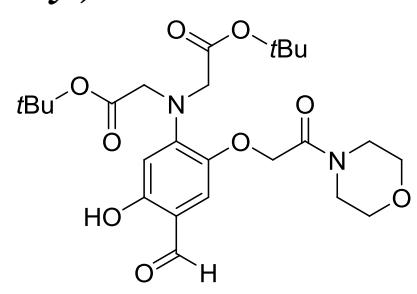

45

$\mathrm{C}_{25} \mathrm{H}_{36} \mathrm{~N}_{2} \mathrm{O}_{9}$

$508.24 \mathrm{~g} / \mathrm{mol}$

Di-tert-butyl 2,2'-((5-(benzyloxy)-4-formyl-2-(2-morpholino-2-oxoethoxy)phenyl)azanediyl)diacetate $(2.52 \mathrm{~g})$ was dissolved in acetic acid $(15.00 \mathrm{~mL} / \mathrm{g}$ educt $)$ and $10 \%$ $\mathrm{Pd} / \mathrm{C}\left(294.10 \mathrm{mg} / \mathrm{g}\right.$ educt) was added. The solution was stirred for up to $2 \mathrm{~h}$ under $\mathrm{H}_{2}$ atmosphere (room pressure). The reaction solution was filtered over celite and the solvent was evaporated under reduced pressure. The crude product was purified by flash column chromatography (pentane/EE, $1: 2 \rightarrow 0: 1$ ). The product was obtained after lyophilisation as white solid (1.57 g, $3.09 \mathrm{mmol}, 60 \%$ over 2 steps).

${ }^{1}$ H-NMR (300 MHz, [D 6 ]DMSO): $\delta(\mathrm{ppm})=1.41(\mathrm{~s}, 18 \mathrm{H},-\mathrm{COO} \underline{\mathrm{Bu}}), 3.43-3.51(\mathrm{~m}, 4 \mathrm{H}$, $-\mathrm{N}-\underline{\mathrm{H}}_{2}-\mathrm{CH}_{2}-\mathrm{O}$ ), $3.52-3.65$ (m, overlaid by dioxane, $\left.-\mathrm{N}^{-} \mathrm{CH}_{2}-\underline{\mathrm{C}}_{2}-\mathrm{O}\right), 4.18$ (s, 4H, -N$\underline{\mathrm{C}}_{2}-\mathrm{COO} t \mathrm{Bu}$ ), 4.77 (s, 2H, CON-C $\underline{H}_{2}-\mathrm{O}$ ), 6.08 (s, 1H, - $\underline{\mathrm{Ph}}-\mathrm{NH}-$ ), 7.07 (s, 1H, - $\underline{\mathrm{Ph}}-\mathrm{NH}-$ ), $9.88(\mathrm{~s}, 1 \mathrm{H}, \mathrm{C} \underline{\mathrm{HO}}), 10.50(\mathrm{~s}, 1 \mathrm{H}, \mathrm{O} \underline{\mathrm{H}})$.

${ }^{13}$ C-NMR (75 MHz, [D 6 DMSO): $\delta(\mathrm{ppm})=28.22,42.06,45.07,55.21,66.81,66.94$, $81.15,103.90,113.13,113.90,142.25,147.47,157.89$, 166.27, 169.61, 189.61 .

MS (ESI, MeOH): m/z = 509.3 $[\mathrm{M}+\mathrm{H}]^{+}, 531.2[\mathrm{M}+\mathrm{Na}]^{+}$.

HR-MS (ESI, MeOH):

$$
\begin{array}{lll}
\mathrm{C}_{25} \mathrm{H}_{37} \mathrm{~N}_{2} \mathrm{O}_{9}[\mathrm{M}+\mathrm{H}]^{+} & \text {calc.: 509.2494 } & \text { mesur.: 509.2492, } \\
\mathrm{C}_{25} \mathrm{H}_{36} \mathrm{~N}_{2} \mathrm{O}_{9} \mathrm{Na}[\mathrm{M}+\mathrm{Na}]^{+} & \text {calc.: } 531.2313 & \text { mesur.: 531.2313. }
\end{array}
$$




\section{Di-tert-butyl 2,2'-((3-(benzothiazol-2-yl)-2-imino-6-(2-morpholino-2- oxoethoxy)-2H-chromen-7-yl)azanediyl)diacetate}

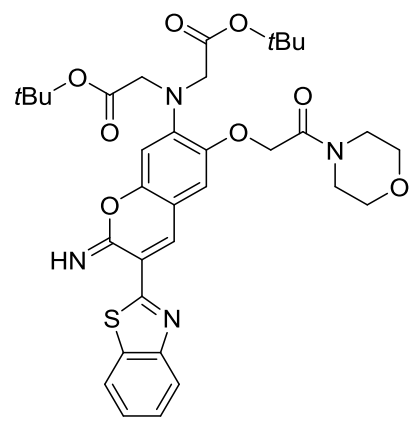

7

$\mathrm{C}_{34} \mathrm{H}_{40} \mathrm{~N}_{4} \mathrm{O}_{8} \mathrm{~S}$

$664.26 \mathrm{~g} / \mathrm{mol}$

To a solution of di-tert-butyl 2,2'-((4-formyl-5-hydroxy-2-(2-morpholino-2oxoethoxy)phenyl)azanediyl)diacetate (1.57 g, $3.09 \mathrm{mmol}, 1.00 \mathrm{eq})$ in dry $\mathrm{MeOH}(10 \mathrm{~mL})$, piperidine $(3.05 \mathrm{~mL}, 30.87 \mathrm{mmol}, 10.00 \mathrm{eq})$ was added. Then Benzothiazole-2-ylacetonitrile (540 mg, $3.09 \mathrm{mmol}, 1.0 \mathrm{eq}$ ) was added and the solution was stirred overnight under argon atmosphere at RT. The precipitate was filtered and washed with dry ether and dried under reduced pressure. The product was obtained by flash column chromatography (pentane/ethanol, 1:1) as orange solid (1.44 g, $2.17 \mathrm{mmol}, 70 \%)$

${ }^{\mathbf{1}} \mathbf{H}-\mathrm{NMR}\left(300 \mathrm{MHz},\left[\mathrm{D}_{6}\right] \mathrm{DMSO}\right): \delta(\mathrm{ppm})=1.38-1.41(\mathrm{~m}, 18 \mathrm{H},-\mathrm{COO} \underline{t \mathrm{Bu}}), 3.41-3.54$ (m, 4H, -N-C $\left.\underline{\mathrm{H}}_{2}-\mathrm{CH}_{2}-\mathrm{O}\right), 3.57-3.69\left(\mathrm{~m}, 4 \mathrm{H},-\mathrm{N}-\mathrm{CH}_{2}-\underline{\mathrm{C}}_{2}-\mathrm{O}\right), 4.15-4.26(\mathrm{~m}, 4 \mathrm{H},-\mathrm{N}-$ $\left.\mathrm{CH}_{2}-\mathrm{COO} t \mathrm{Bu}\right), 4.87$ (s, 2H, CON-C $\left.\underline{H}_{2}-\mathrm{O}\right), 6.42$ (s, 1H, $\left.-\underline{\mathrm{Ph}}-\mathrm{NH}-\right), 7.30-7.65$ (m, 3H,

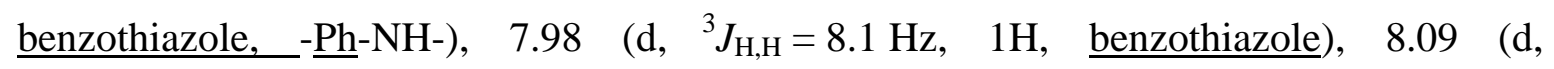
${ }^{3} J_{\mathrm{H}, \mathrm{H}}=7.8 \mathrm{~Hz}, 1 \mathrm{H}$, benzothiazol $), 8.63\left(\mathrm{bs}, 1 \mathrm{H}, \mathrm{H}_{\mathrm{Ar}}\right), 8.72(\mathrm{bs}, 1 \mathrm{H},=\mathrm{NH})$

${ }^{13}$ C-NMR (75 MHz, [D $]$ DMSO): $\delta(p p m)=28.24,42.03,44.92,55.13,66.54,81.15$, $103.25,110.88,112.64,117.82,122.30,122.42$, 125.02, 126.60, 135.93, 137.08, 143.83, $145.37,149.72,152.30,154.53,165.97,169.83$.

MS (ESI, MeOH): m/z = $665.3[\mathrm{M}+\mathrm{H}]^{+}, 1329.5[2 \mathrm{M}+\mathrm{H}]^{+}$.

HR-MS (ESI, MeOH):

$$
\mathrm{C}_{34} \mathrm{H}_{41} \mathrm{~N}_{4} \mathrm{O}_{8 \mathrm{~S}}[\mathrm{M}+\mathrm{H}]^{+} \quad \text { calc.: } 665.2640 \quad \text { mesur.: 665.2641. }
$$


(Z/E)-3-((3-(Benzothiazol-2-yl)-7-(bis(2-(tert-butoxy)-2-oxoethyl)amino)-6-(2morpholino-2-oxoethoxy)-2H-chromen-2-ylidene)amino)propanoic acid

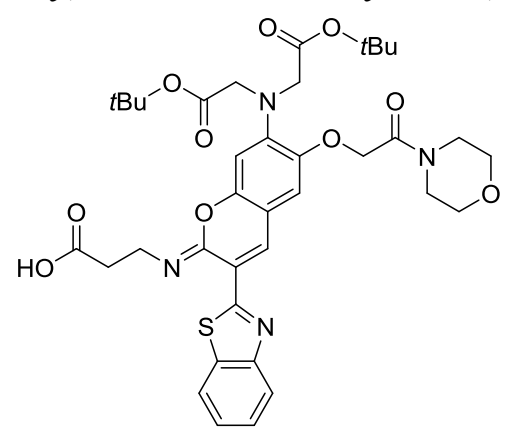

46

$\mathrm{C}_{37} \mathrm{H}_{44} \mathrm{~N}_{4} \mathrm{O}_{10} \mathrm{~S}$

$736.28 \mathrm{~g} / \mathrm{mol}$

Following SOP 846 was achieved with di-tert-butyl 2,2'-((3-(benzothiazol-2-yl)-6-(2-(2(bis(2-(tert-butoxy)-2-oxoethyl)amino)phenoxy)ethoxy)-2-imino-2H-chromen-7-

yl)azanediyl)diacetate $\quad(1.14 \mathrm{~g}, \quad 1.71 \mathrm{mmol}, \quad 1.00 \mathrm{eq})$ and 3-aminopropanoic acid hydrochloride $(215 \mathrm{mg}, 1.71 \mathrm{mmol}, 1.00 \mathrm{eq})$. The crude product was purified by flash column chromatography (pentane/EE, 1:2 $\rightarrow 0: 1$ ). The product was obtained after lyophilisation as red solid (745 mg, $1.01 \mathrm{mmol}, 59.0 \%)$.

${ }^{1}$ H-NMR (300 MHz, [D 6 DMSO): $\delta(\mathrm{ppm})=1.38-1.51(\mathrm{~m}, 18 \mathrm{H},-\mathrm{COO} \underline{\mathrm{Bu}}), 2.67-2.81$ (m, $2 \mathrm{H}, \mathrm{N}-\underline{\mathrm{C}}_{2}-\mathrm{CH}_{2}-\mathrm{COOH}$ ), $3.50-3.857$ (m, 2H, N-CH${ }_{2}-\underline{\mathrm{CH}}_{2}-\mathrm{COOH}$ ), $3.58-3-76$ (m, $\left.8 \mathrm{H},-\mathrm{N}-\underline{\mathrm{H}}_{2}-\mathrm{CH}_{2}-\mathrm{O},-\mathrm{N}-\mathrm{CH}_{2}-\underline{\mathrm{C}}_{2}-\mathrm{O}\right), 4.02-4.14\left(\mathrm{~m}, 4 \mathrm{H},-\mathrm{N}-\underline{\mathrm{C}}_{2}-\mathrm{COO} t \mathrm{Bu}\right), 4.71-4.78$ (m, 2H, CON-C $\underline{\mathrm{H}}_{2}-\mathrm{O}$ ), 6.39 (s, 1H, - $\left.\underline{\mathrm{Ph}}-\mathrm{NH}-\right), 6.92$ (s, 1H, - $\left.\underline{\mathrm{Ph}}-\mathrm{NH}-\right), 7.10-7.36$ (m, 3H, benzothiazole $), 7.79\left(\mathrm{dd},{ }^{3} J_{\mathrm{H}, \mathrm{H}}=8.0,25.1 \mathrm{~Hz}, 2 \mathrm{H}\right.$, benzothiazole $), 8.15\left(\mathrm{~s}, 1 \mathrm{H}, \mathrm{H}_{\mathrm{Ar}}\right)$.

${ }^{13}$ C-NMR (126 MHz, [D $]$ DMSO): $\delta(\mathrm{ppm})=28.23,42.17,42.40,45.49,54.75,66.74$, $66.83,68.53,81.84,104.81,112.10,113.93,121.28,122.36,124.57,125.82,136.60$, $143.75,145.75,149.44,151.98,161.31,166.14,169.48$.

MS (ESI, MeOH): m/z = $737.3[\mathrm{M}+\mathrm{H}]^{+}$.

HR-MS (ESI, MeOH):
$\mathrm{C}_{37} \mathrm{H}_{45} \mathrm{~N}_{4} \mathrm{O}_{10} \mathrm{~S}[\mathrm{M}+\mathrm{H}]^{+}$
calc.: 737.2851
mesur.: 737.2844;
$\mathrm{C}_{37} \mathrm{H}_{44} \mathrm{~N}_{4} \mathrm{O}_{10} \mathrm{Na}[\mathrm{M}+\mathrm{Na}]^{+} \quad$ calc.: 759.2670
mesur.: 759.2668 . 


\subsection{6 $\beta$-Peptides}

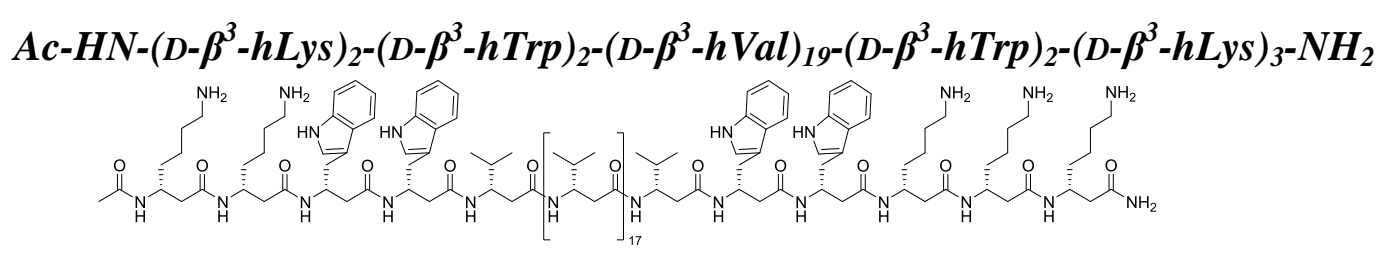

\section{Pep Met 1}

$$
\begin{aligned}
& \mathrm{C}_{199} \mathrm{H}_{332} \mathrm{~N}_{38} \mathrm{O}_{29} \\
& 3718.57 \mathrm{~g} / \mathrm{mol}
\end{aligned}
$$

The peptide Pep Met 1 was synthesised following SOP 6 using the amino acids described in Chapter 6.5.1.

MS (ESI, MeOH): m/z = 1241.2 $[\mathrm{M}+3 \mathrm{H}]^{3+}, 931.1[\mathrm{M}+4 \mathrm{H}]^{4+}, 745.1[\mathrm{M}+5 \mathrm{H}]^{5+}, 621.1$ $[\mathrm{M}+6 \mathrm{H}]^{6+}$.

HR-MS (ESI, MeOH):

$\mathrm{C}_{199} \mathrm{H}_{335} \mathrm{~N}_{38} \mathrm{O}_{29}[\mathrm{M}+3 \mathrm{H}]^{3+} \quad$ calculated: $1241.1984 \quad$ measured: 1241.2000;

$\mathrm{C}_{199} \mathrm{H}_{336} \mathrm{~N}_{38} \mathrm{O}_{29}[\mathrm{M}+4 \mathrm{H}]^{4+} \quad$ calculated: $931.1506 \quad$ measured: 931.1521;

$\mathrm{C}_{199} \mathrm{H}_{337} \mathrm{~N}_{38} \mathrm{O}_{29}[\mathrm{M}+5 \mathrm{H}]^{5+} \quad$ calculated: $745.1219 \quad$ measured: 745.1233;

$\mathrm{C}_{199} \mathrm{H}_{33} \mathrm{~N}_{38} \mathrm{O}_{29}[\mathrm{M}+6 \mathrm{H}]^{6+} \quad$ calculated: $621.1028 \quad$ measured: 621.1035.

HPLC (analytic, A $\left(\mathrm{H}_{2} \mathrm{O}+0.1 \%\right.$ TFA + 0.1 HFIP $), \mathrm{B}(\mathrm{MeOH}+0.1 \% \mathrm{TFA}+0.1 \mathrm{HFIP})$, $\left.80.0 \rightarrow 100.0 \% \mathrm{~B}, 30 \mathrm{~min}, 50{ }^{\circ} \mathrm{C}\right): t_{\mathrm{R}}=14.4 \mathrm{~min}$.

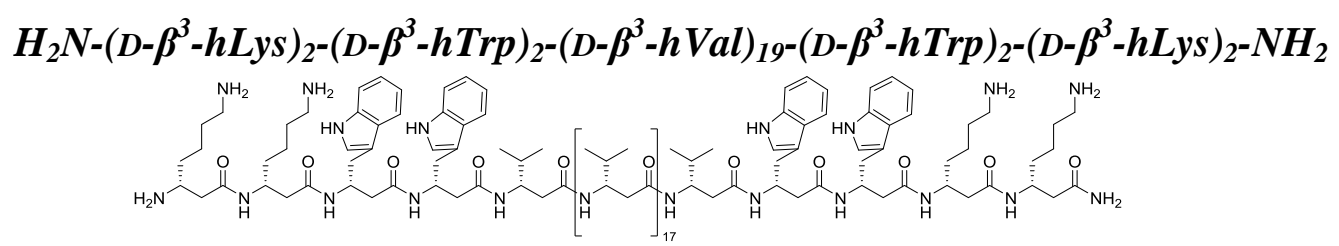

\section{Pep Met 2}

$\mathrm{C}_{190} \mathrm{H}_{316} \mathrm{~N}_{36} \mathrm{O}_{29}$

$3534.45 \mathrm{~g} / \mathrm{mol}$

The peptide Pep Met 2 was synthesised following SOP 6 using the amino acids described in Chapter 6.5.1.

MS (ESI, MeOH): m/z = $1179.8[\mathrm{M}+3 \mathrm{H}]^{3+}, 885.1[\mathrm{M}+4 \mathrm{H}]^{4+}, 708.3[\mathrm{M}+5 \mathrm{H}]^{5+}, 590.4$ $[\mathrm{M}+6 \mathrm{H}]^{6+}$. 
HR-MS (ESI, MeOH):
$\mathrm{C}_{190} \mathrm{H}_{318} \mathrm{~N}_{36} \mathrm{O}_{29}[\mathrm{M}+2 \mathrm{H}]^{2+} \quad$ calculated: 1769.2333
measured: 1769.2345;
$\mathrm{C}_{190} \mathrm{H}_{319} \mathrm{~N}_{36} \mathrm{O}_{29}[\mathrm{M}+3 \mathrm{H}]^{3+} \quad$ calculated: 1179.8246
measured: 1179.8267;
$\mathrm{C}_{190} \mathrm{H}_{320} \mathrm{~N}_{36} \mathrm{O}_{29}[\mathrm{M}+4 \mathrm{H}]^{4+} \quad$ calculated: 885.1203
measured: 885.1218;
$\mathrm{C}_{190} \mathrm{H}_{321} \mathrm{~N}_{36} \mathrm{O}_{29}[\mathrm{M}+5 \mathrm{H}]^{5+} \quad$ calculated: 708.2977
measured: 708.2987;
$\mathrm{C}_{190} \mathrm{H}_{322} \mathrm{~N}_{36} \mathrm{O}_{29}[\mathrm{M}+6 \mathrm{H}]^{6+}$ calculated: 590.4160
measured: 590.4164 .

HPLC (analytic, A $\left(\mathrm{H}_{2} \mathrm{O}+0.1 \%\right.$ TFA $\left.+0.1 \mathrm{HFIP}\right), \mathrm{B}(\mathrm{MeOH}+0.1 \%$ TFA + $0.1 \mathrm{HFIP})$, $\left.80.0 \rightarrow 100.0 \% \mathrm{~B}, 30 \mathrm{~min}, 50{ }^{\circ} \mathrm{C}\right): t_{\mathrm{R}}=15.8 \mathrm{~min}$.

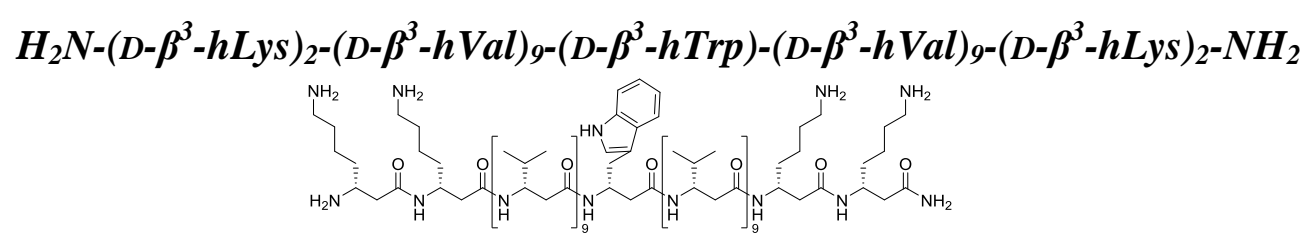

\section{Pep 1}

$\mathrm{C}_{148} \mathrm{H}_{269} \mathrm{~N}_{29} \mathrm{O}_{23}$

$2821.08 \mathrm{~g} / \mathrm{mol}$

The peptide Pep 1 was synthesised following SOP 5 using the amino acids described in Chapter 6.5.1.

MS (ESI, MeOH): $\mathrm{m} / \mathrm{z}=1412.1[\mathrm{M}+2 \mathrm{H}]^{2+}, 941.7[\mathrm{M}+3 \mathrm{H}]^{3+}, 706.5[\mathrm{M}+4 \mathrm{H}]^{4+}, 565.4$ $[\mathrm{M}+5 \mathrm{H}]^{5+}$.

\section{HR-MS (ESI, MeOH):}

$\begin{array}{lll}\mathrm{C}_{148} \mathrm{H}_{271} \mathrm{~N}_{29} \mathrm{O}_{23}[\mathrm{M}+2 \mathrm{H}]^{2+} & \text { calculated: } 1412.0473 & \text { measured: 1412.0488; } \\ \mathrm{C}_{148} \mathrm{H}_{272} \mathrm{~N}_{29} \mathrm{O}_{23}[\mathrm{M}+3 \mathrm{H}]^{3+} & \text { calculated: } 941.7007 & \text { measured: 941.7014; } \\ \mathrm{C}_{148} \mathrm{H}_{273} \mathrm{~N}_{29} \mathrm{O}_{23}[\mathrm{M}+4 \mathrm{H}]^{4+} & \text { calculated: } 706.5273 & \text { measured: 706.5281; } \\ \mathrm{C}_{148} \mathrm{H}_{274} \mathrm{~N}_{29} \mathrm{O}_{23}[\mathrm{M}+5 \mathrm{H}]^{5+} & \text { calculated: } 565.4233 & \text { measured: } 565.4235 .\end{array}$

HPLC (preparative, A $\left(\mathrm{H}_{2} \mathrm{O}+0.1 \%\right.$ TFA), B $(\mathrm{MeOH}+0.1 \%$ TFA $), 90.0 \rightarrow 100.0 \% \mathrm{~B}$, $30 \min , \mathrm{RT}): t_{\mathrm{R}}=8.6 \mathrm{~min}$. 


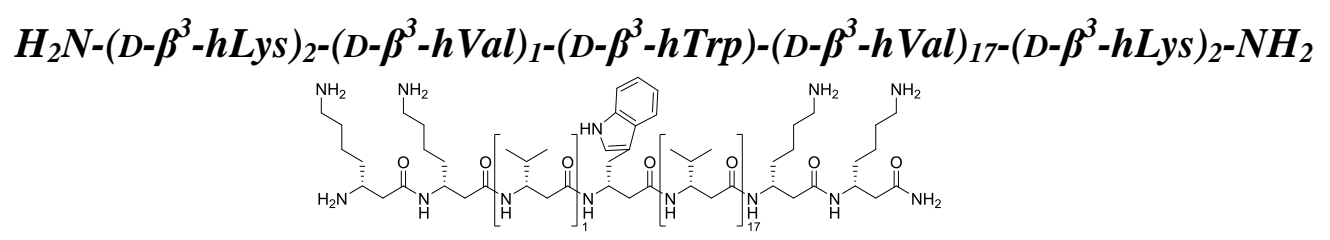

Pep 2

$\mathrm{C}_{148} \mathrm{H}_{269} \mathrm{~N}_{29} \mathrm{O}_{23}$

$2821.08 \mathrm{~g} / \mathrm{mol}$

The peptide Pep 2 was synthesised following SOP 5 using the amino acids described in Chapter 6.5.1.

MS (ESI, MeOH): m/z = $1412.0[\mathrm{M}+2 \mathrm{H}]^{2+}, 941.7[\mathrm{M}+3 \mathrm{H}]^{3+}, 706.5[\mathrm{M}+4 \mathrm{H}]^{4+}, 565.4$ $[\mathrm{M}+5 \mathrm{H}]^{5+}$.

HR-MS (ESI, MeOH):

$\mathrm{C}_{148} \mathrm{H}_{271} \mathrm{~N}_{29} \mathrm{O}_{23}[\mathrm{M}+2 \mathrm{H}]^{2+} \quad$ calculated: $1412.0473 \quad$ measured: 1412.0475;

$\mathrm{C}_{148} \mathrm{H}_{272} \mathrm{~N}_{29} \mathrm{O}_{23}[\mathrm{M}+3 \mathrm{H}]^{3+} \quad$ calculated: $941.7007 \quad$ measured: 941.7015;

$\mathrm{C}_{148} \mathrm{H}_{273} \mathrm{~N}_{29} \mathrm{O}_{23}[\mathrm{M}+4 \mathrm{H}]^{4+} \quad$ calculated: $706.5273 \quad$ measured: 706.5277;

$\mathrm{C}_{148} \mathrm{H}_{274} \mathrm{~N}_{29} \mathrm{O}_{23}[\mathrm{M}+5 \mathrm{H}]^{5+} \quad$ calculated: $565.4233 \quad$ measured: 565.4234 .

HPLC (preparative, A $\left(\mathrm{H}_{2} \mathrm{O}+0.1 \%\right.$ TFA), $\mathrm{B}(\mathrm{MeOH}+0.1 \%$ TFA $), 90.0 \rightarrow 100.0 \% \mathrm{~B}$, $30 \min , \mathrm{RT}): t_{\mathrm{R}}=9.8 \mathrm{~min}$.

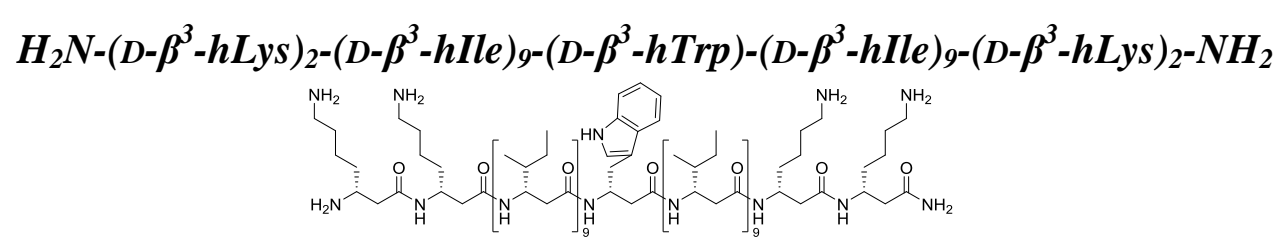

Pep 3

$\mathrm{C}_{166} \mathrm{H}_{305} \mathrm{~N}_{29} \mathrm{O}_{23}$

$3073.36 \mathrm{~g} / \mathrm{mol}$

The peptide Pep 3 was synthesised following SOP 5 using the amino acids described in Chapter 6.5.1.

MS (ESI, MeOH): m/z = $1538.7[\mathrm{M}+2 \mathrm{H}]^{2+}, 1025.8[\mathrm{M}+3 \mathrm{H}]^{3+}, 769.6[\mathrm{M}+4 \mathrm{H}]^{4+}, 615.9$ $[\mathrm{M}+5 \mathrm{H}]^{5+}$. 
HR-MS (ESI, MeOH):
$\mathrm{C}_{166} \mathrm{H}_{307} \mathrm{~N}_{29} \mathrm{O}_{23}[\mathrm{M}+3 \mathrm{H}]^{3+} \quad$ calculated: 1025.7946
measured: 1025.7949;
$\mathrm{C}_{166} \mathrm{H}_{308} \mathrm{~N}_{29} \mathrm{O}_{23}[\mathrm{M}+4 \mathrm{H}]^{4+} \quad$ calculated: 769.5977
measured: 769.5983;
$\mathrm{C}_{166} \mathrm{H}_{309} \mathrm{~N}_{29} \mathrm{O}_{23}[\mathrm{M}+5 \mathrm{H}]^{5+} \quad$ calculated: 615.8797
measured: 615.8797.

HPLC (preparative, A $\left(\mathrm{H}_{2} \mathrm{O}+0.1 \%\right.$ TFA $), \mathrm{B}(\mathrm{MeOH}+0.1 \%$ TFA $), 92.0 \rightarrow 100.0 \% \mathrm{~B}$, $30 \mathrm{~min}, \mathrm{RT}): t_{\mathrm{R}}=10.7 \mathrm{~min}$.

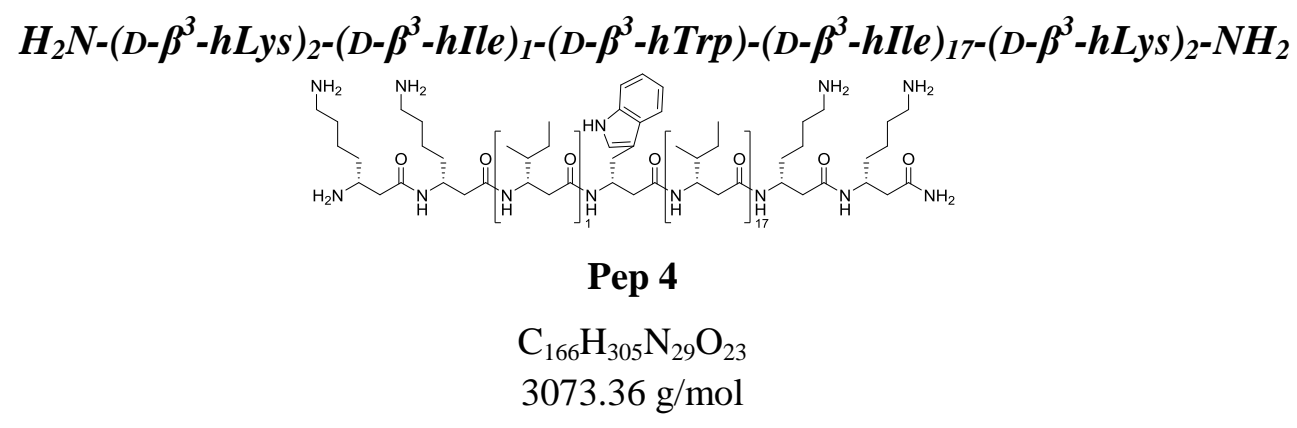

The peptide Pep 4 was synthesised following SOP 5 using the amino acids described in Chapter 6.5.1.

MS (ESI, MeOH): m/z = 1538.2 $[\mathrm{M}+2 \mathrm{H}]^{2+}, 1025.8[\mathrm{M}+3 \mathrm{H}]^{3+}, 769.6[\mathrm{M}+4 \mathrm{H}]^{4+}, 616.1$ $[\mathrm{M}+5 \mathrm{H}]^{5+}$.

\section{HR-MS (ESI, MeOH):}

$\mathrm{C}_{166} \mathrm{H}_{307} \mathrm{~N}_{29} \mathrm{O}_{23}[\mathrm{M}+2 \mathrm{H}]^{2+} \quad$ calculated: $1538.1882 \quad$ measured: 1538.1895;

$\mathrm{C}_{166} \mathrm{H}_{308} \mathrm{~N}_{29} \mathrm{O}_{23}[\mathrm{M}+3 \mathrm{H}]^{3+} \quad$ calculated: $1025.7946 \quad$ measured: 1025.7952;

$\mathrm{C}_{166} \mathrm{H}_{309} \mathrm{~N}_{29} \mathrm{O}_{23}[\mathrm{M}+4 \mathrm{H}]^{4+} \quad$ calculated: 769.5977 measured: 769.5991;

$\mathrm{C}_{166} \mathrm{H}_{310} \mathrm{~N}_{29} \mathrm{O}_{23}[\mathrm{M}+5 \mathrm{H}]^{5+} \quad$ calculated: $615.8797 \quad$ measured: 615.8815 .

HPLC (preparative, A $\left(\mathrm{H}_{2} \mathrm{O}+0.1 \%\right.$ TFA $), \mathrm{B}(\mathrm{MeOH}+0.1 \%$ TFA $), 92.0 \rightarrow 98.0 \% \mathrm{~B}$, 30 min, $\mathrm{RT}$ ): $t_{\mathrm{R}}=16.3 \mathrm{~min}$. 


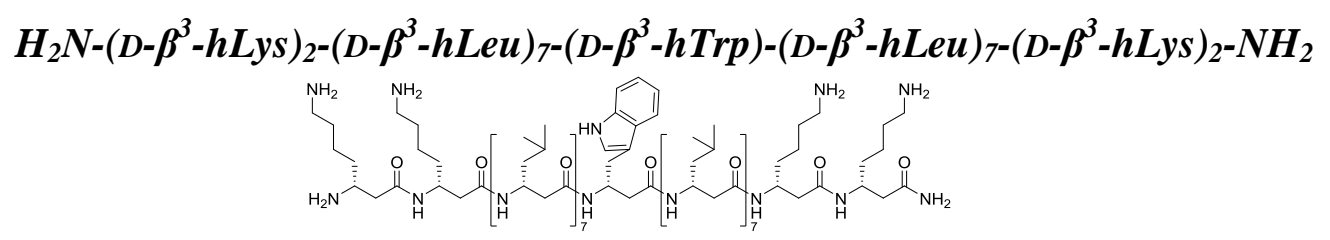

Pep 5

$\mathrm{C}_{138} \mathrm{H}_{253} \mathrm{~N}_{25} \mathrm{O}_{19}$

$2564.96 \mathrm{~g} / \mathrm{mol}$

The peptide Pep 5 was synthesised following SOP 5 using the amino acids described in Chapter 6.5.1.

MS (ESI, MeOH): m/z = $1284.0[\mathrm{M}+2 \mathrm{H}]^{2+}, 856.3[\mathrm{M}+3 \mathrm{H}]^{3+}, 642.5[\mathrm{M}+4 \mathrm{H}]^{4+}, 514.2$ $[\mathrm{M}+5 \mathrm{H}]^{5+}$.

HR-MS (ESI, MeOH):

$\mathrm{C}_{138} \mathrm{H}_{255} \mathrm{~N}_{25} \mathrm{O}_{19}[\mathrm{M}+2 \mathrm{H}]^{2+} \quad$ calculated: $1283.9888 \quad$ measured: 1283.9893;

$\mathrm{C}_{138} \mathrm{H}_{256} \mathrm{~N}_{25} \mathrm{O}_{19}[\mathrm{M}+3 \mathrm{H}]^{3+} \quad$ calculated: $856.3283 \quad$ measured: 856.3288;

$\mathrm{C}_{138} \mathrm{H}_{257} \mathrm{~N}_{25} \mathrm{O}_{19}[\mathrm{M}+4 \mathrm{H}]^{4+} \quad$ calculated: $642.4980 \quad$ measured: 642.4987;

$\mathrm{C}_{138} \mathrm{H}_{258} \mathrm{~N}_{25} \mathrm{O}_{19}[\mathrm{M}+5 \mathrm{H}]^{5+} \quad$ calculated: $514.1999 \quad$ measured: 514.1998.

HPLC (preparative, A $\left(\mathrm{H}_{2} \mathrm{O}+0.1 \%\right.$ TFA), B $(\mathrm{MeOH}+0.1 \%$ TFA $), 87.0 \rightarrow 95.0 \% \mathrm{~B}$, $40 \min , \mathrm{RT}): t_{\mathrm{R}}=20.5 \mathrm{~min}$.

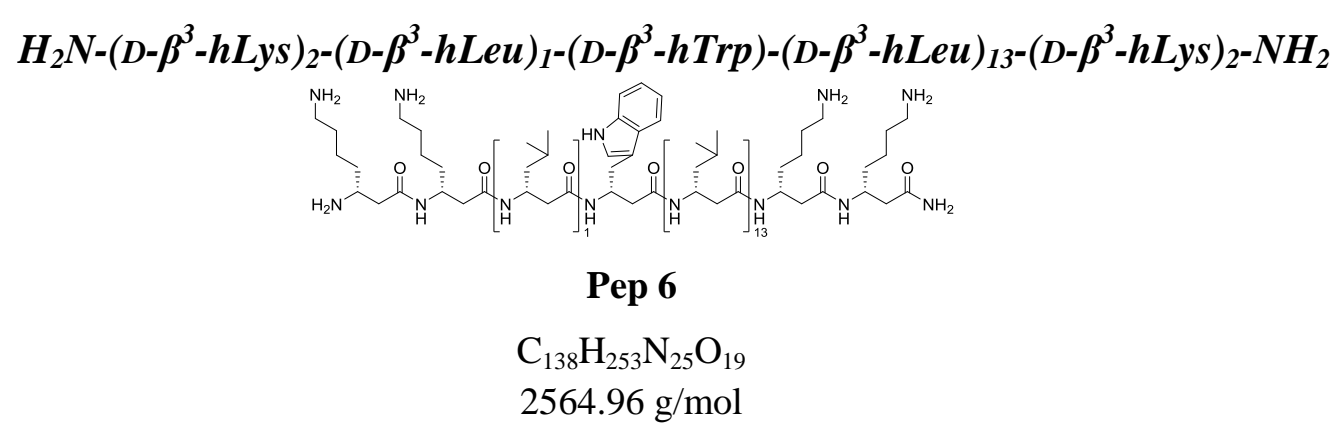

The peptide Pep 6 was synthesised following SOP 5 using the amino acids described in Chapter 6.5.1.

MS (ESI, MeOH): $\mathrm{m} / \mathrm{z}=1284.0[\mathrm{M}+2 \mathrm{H}]^{2+}, 856.3[\mathrm{M}+3 \mathrm{H}]^{3+}, 642.5[\mathrm{M}+4 \mathrm{H}]^{4+}, 514.2$ $[\mathrm{M}+5 \mathrm{H}]^{5+}$. 
HR-MS (ESI, MeOH):
$\mathrm{C}_{138} \mathrm{H}_{255} \mathrm{~N}_{25} \mathrm{O}_{19}[\mathrm{M}+2 \mathrm{H}]^{2+} \quad$ calculated: 1283.9888
measured: 1283.9888;
$\mathrm{C}_{138} \mathrm{H}_{256} \mathrm{~N}_{25} \mathrm{O}_{19}[\mathrm{M}+3 \mathrm{H}]^{3+} \quad$ calculated: 856.3283
measured: 856.3283;
$\mathrm{C}_{138} \mathrm{H}_{257} \mathrm{~N}_{25} \mathrm{O}_{19}[\mathrm{M}+4 \mathrm{H}]^{4+}$ calculated: 642.4980
measured: 642.4978;
$\mathrm{C}_{138} \mathrm{H}_{258} \mathrm{~N}_{25} \mathrm{O}_{19}[\mathrm{M}+5 \mathrm{H}]^{5+} \quad$ calculated: 514.1999
measured: 514.1989.

HPLC (preparative, A $\left(\mathrm{H}_{2} \mathrm{O}+0.1 \%\right.$ TFA $), \mathrm{B}(\mathrm{MeOH}+0.1 \%$ TFA $), 85.0 \rightarrow 95.0 \% \mathrm{~B}$, 40 min, $\mathrm{RT}): t_{\mathrm{R}}=28.9 \mathrm{~min}$.

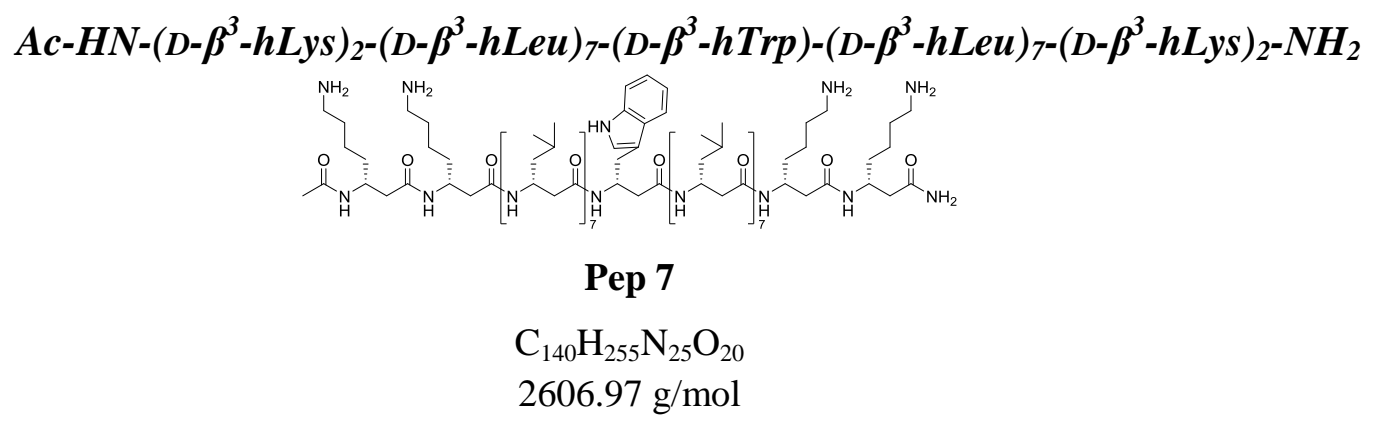

The peptide Pep 7 was synthesised following SOP 5 using the amino acids described in Chapter 6.5.1.

MS (ESI, MeOH): m/z = 1305.0 $[\mathrm{M}+2 \mathrm{H}]^{2+}, 870.3[\mathrm{M}+3 \mathrm{H}]^{3+}, 653.0[\mathrm{M}+4 \mathrm{H}]^{4+}$.

\section{HR-MS (ESI, MeOH):}
$\mathrm{C}_{140} \mathrm{H}_{257} \mathrm{~N}_{25} \mathrm{O}_{20}[\mathrm{M}+2 \mathrm{H}]^{2+} \quad$ calculated: 1304.9941
measured: 1304.9937;
$\mathrm{C}_{140} \mathrm{H}_{258} \mathrm{~N}_{25} \mathrm{O}_{20}[\mathrm{M}+3 \mathrm{H}]^{3+} \quad$ calculated: 870.3318 measured: 870.3311;
$\mathrm{C}_{140} \mathrm{H}_{259} \mathrm{~N}_{25} \mathrm{O}_{20}[\mathrm{M}+4 \mathrm{H}]^{4+} \quad$ calculated: 653.0007
measured: 652.9996 .

HPLC (preparative, A $\left(\mathrm{H}_{2} \mathrm{O}+0.1 \%\right.$ TFA), $\mathrm{B}(\mathrm{MeOH}+0.1 \%$ TFA $), 90.0 \rightarrow 100.0 \% \mathrm{~B}$, $90 \min , \mathrm{RT}): t_{\mathrm{R}}=14.4 \mathrm{~min}$. 


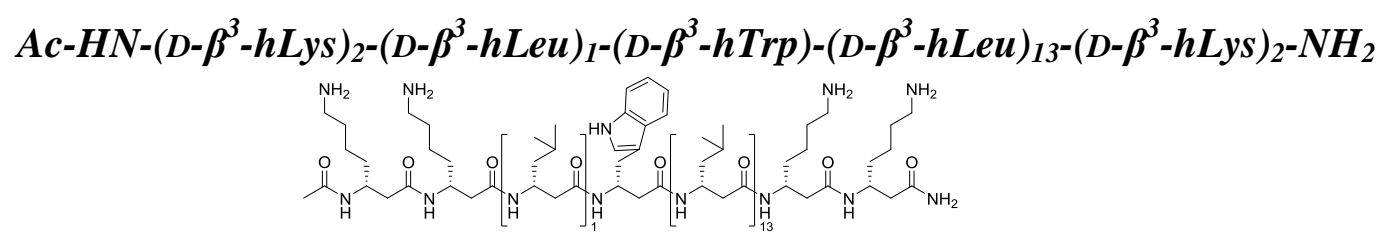

Pep 8

$\mathrm{C}_{140} \mathrm{H}_{255} \mathrm{~N}_{25} \mathrm{O}_{20}$

$2606.97 \mathrm{~g} / \mathrm{mol}$

The peptide Pep 8 was synthesised following SOP 5 using the amino acids described in Chapter 6.5.1.

MS (ESI, MeOH): m/z = $1304.99[\mathrm{M}+2 \mathrm{H}]^{2+}, 870.33[\mathrm{M}+3 \mathrm{H}]^{3+}, 653.00[\mathrm{M}+4 \mathrm{H}]^{4+}, 522.60$ $[\mathrm{M}+5 \mathrm{H}]^{5+}$.

HR-MS (ESI, MeOH):

$\mathrm{C}_{140} \mathrm{H}_{257} \mathrm{~N}_{25} \mathrm{O}_{20}[\mathrm{M}+2 \mathrm{H}]^{2+} \quad$ calculated: $1304.9941 \quad$ measured: 1304.9950;

$\mathrm{C}_{140} \mathrm{H}_{258} \mathrm{~N}_{25} \mathrm{O}_{20}[\mathrm{M}+3 \mathrm{H}]^{3+} \quad$ calculated: $870.3318 \quad$ measured: 870.3326;

$\mathrm{C}_{140} \mathrm{H}_{259} \mathrm{~N}_{25} \mathrm{O}_{20}[\mathrm{M}+4 \mathrm{H}]^{4+} \quad$ calculated: $653.0007 \quad$ measured: 653.0013 .

HPLC (preparative, A $\left(\mathrm{H}_{2} \mathrm{O}+0.1 \%\right.$ TFA), B $(\mathrm{MeOH}+0.1 \%$ TFA $), 87.0 \rightarrow 95.0 \% \mathrm{~B}$, $40 \min , \mathrm{RT}): t_{\mathrm{R}}=20.6 \mathrm{~min}$.

\section{$H_{2} N-\left(D-\beta^{3}-h L y s\right)_{2}-\left(D-\beta^{3}-h L e u-D-\beta^{3}-h L e u-(R, R)-a c h c\right)_{3}-\left(D-\beta^{3}-h T r p\right)-\left(D-\beta^{3}-h L e u-\right.$} $\left.(R, R)-a c h c-D-h \beta^{3}-L e u\right)_{3}-\left(D-\beta^{3}-h L y s\right)_{2}-N_{2}$

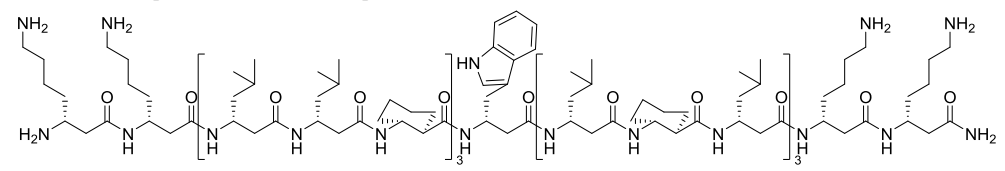

Pep 9

$\mathrm{C}_{166} \mathrm{H}_{293} \mathrm{~N}_{29} \mathrm{O}_{23}$

$3061.26 \mathrm{~g} / \mathrm{mol}$

The peptide Pep 9 was synthesised following SOP 5 using the amino acids described in Chapter 6.5.1.

MS (ESI, MeOH): $\mathrm{m} / \mathrm{z}=1532.2[\mathrm{M}+2 \mathrm{H}]^{2+}, 1022.1[\mathrm{M}+3 \mathrm{H}]^{3+}, 766.6[\mathrm{M}+4 \mathrm{H}]^{4+}, 613.7$ $[\mathrm{M}+5 \mathrm{H}]^{5+}$. 
HR-MS (ESI, MeOH):

$\begin{array}{lll}\mathrm{C}_{166} \mathrm{H}_{295} \mathrm{~N}_{29} \mathrm{O}_{23}[\mathrm{M}+2 \mathrm{H}]^{2+} & \text { calculated: } 1532.1413 & \text { measured: 1532.1408; } \\ \mathrm{C}_{166} \mathrm{H}_{296} \mathrm{~N}_{29} \mathrm{O}_{23}[\mathrm{M}+3 \mathrm{H}]^{3+} & \text { calculated: } 1021.7633 & \text { measured: 1021.7637; } \\ \mathrm{C}_{166} \mathrm{H}_{297} \mathrm{~N}_{29} \mathrm{O}_{23}[\mathrm{M}+4 \mathrm{H}]^{4+} & \text { calculated: 766.5743 } & \text { measured: 766.5753; } \\ \mathrm{C}_{166} \mathrm{H}_{298} \mathrm{~N}_{29} \mathrm{O}_{23}[\mathrm{M}+5 \mathrm{H}]^{5+} & \text { calculated: } 613.4609 & \text { measured: 613.4613. }\end{array}$

HPLC (preparative, A $\left(\mathrm{H}_{2} \mathrm{O}+0.1 \%\right.$ TFA), B $(\mathrm{MeOH}+0.1 \%$ TFA $), 92.0 \rightarrow 98.0 \% \mathrm{~B}$, $30 \min , \mathrm{RT}): t_{\mathrm{R}}=11.7 \mathrm{~min}$.
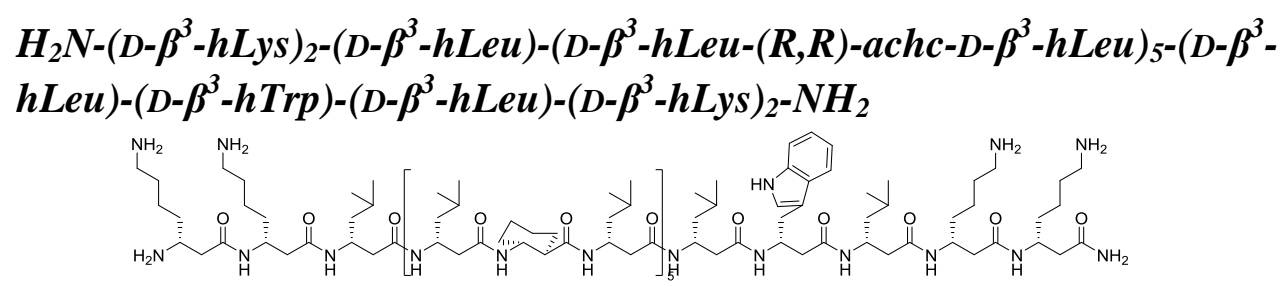

Pep 10

$\mathrm{C}_{166} \mathrm{H}_{295} \mathrm{~N}_{29} \mathrm{O}_{23}$

$3063.28 \mathrm{~g} / \mathrm{mol}$

The peptide Pep 10 was synthesised following SOP 5 using the amino acids described in Chapter 6.5.1.

MS (ESI, MeOH): m/z = $1533.7[\mathrm{M}+2 \mathrm{H}]^{2+}, 1022.4[\mathrm{M}+3 \mathrm{H}]^{3+}, 767.1[\mathrm{M}+4 \mathrm{H}]^{4+}, 613.9$ $[\mathrm{M}+5 \mathrm{H}]^{5+}$.

HR-MS (ESI, MeOH):

$\mathrm{C}_{166} \mathrm{H}_{298} \mathrm{~N}_{29} \mathrm{O}_{23}[\mathrm{M}+3 \mathrm{H}]^{3+} \quad$ calculated: $1022.4352 \quad$ measured: 1022.4364;

$\mathrm{C}_{166} \mathrm{H}_{299} \mathrm{~N}_{29} \mathrm{O}_{23}[\mathrm{M}+4 \mathrm{H}]^{4+} \quad$ calculated: 767.0782 measured: 767.0780;

$\mathrm{C}_{166} \mathrm{H}_{300} \mathrm{~N}_{29} \mathrm{O}_{23}[\mathrm{M}+5 \mathrm{H}]^{5+}$ calculated: $613.8640 \quad$ measured: 613.8644 .

HPLC (preparative, A $\left(\mathrm{H}_{2} \mathrm{O}+0.1 \%\right.$ TFA), B $(\mathrm{MeOH}+0.1 \%$ TFA $), 93.0 \rightarrow 97.0 \% \mathrm{~B}$, 30 min, $\mathrm{RT}$ ): $t_{\mathrm{R}}=11.9$ min. 

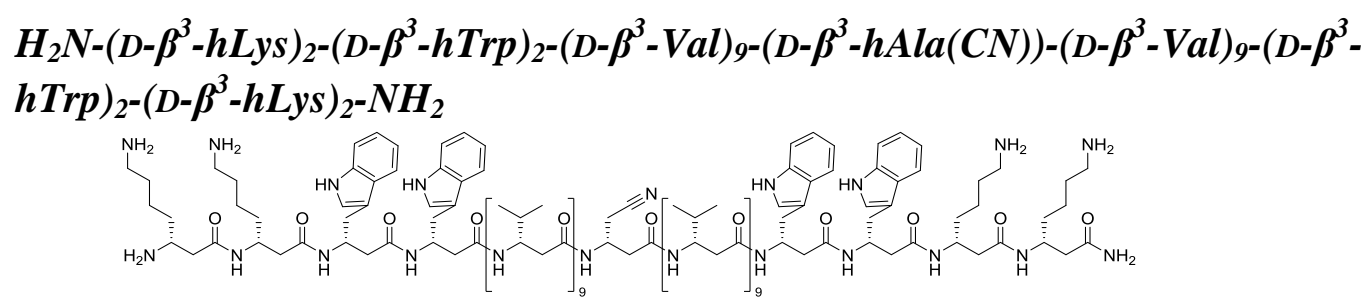

Pep 11

$\mathrm{C}_{189} \mathrm{H}_{311} \mathrm{~N}_{37} \mathrm{O}_{27}$

$3531.41 \mathrm{~g} / \mathrm{mol}$

The peptide Pep 11 was synthesised following SOP 5 using the amino acids described in Chapter 6.5.1.

MS (ESI, MeOH): m/z = $1767.8[\mathrm{M}+2 \mathrm{H}]^{2+}, 1178.9[\mathrm{M}+3 \mathrm{H}]^{3+}, 884.4[\mathrm{M}+4 \mathrm{H}]^{4+}, 707.7$ $[\mathrm{M}+5 \mathrm{H}]^{5+}$.

HR-MS (ESI, MeOH):

$\mathrm{C}_{189} \mathrm{H}_{313} \mathrm{~N}_{37} \mathrm{O}_{27}[\mathrm{M}+2 \mathrm{H}]^{2+} \quad$ calculated: $1767.7153 \quad$ measured: 1767.7143;

$\mathrm{C}_{189} \mathrm{H}_{314} \mathrm{~N}_{37} \mathrm{O}_{27}[\mathrm{M}+3 \mathrm{H}]^{3+} \quad$ calculated: $1178.8126 \quad$ measured: 1178.8141;

$\mathrm{C}_{189} \mathrm{H}_{315} \mathrm{~N}_{37} \mathrm{O}_{27}[\mathrm{M}+4 \mathrm{H}]^{4+} \quad$ calculated: $884.3613 \quad$ measured: 884.3626;

$\mathrm{C}_{189} \mathrm{H}_{316} \mathrm{~N}_{37} \mathrm{O}_{27}[\mathrm{M}+5 \mathrm{H}]^{5+} \quad$ calculated: 707.6905 measured: 707.6915.

HPLC (preparative, A $\left(\mathrm{H}_{2} \mathrm{O}+0.1 \%\right.$ TFA), B $(\mathrm{MeOH}+0.1 \%$ TFA $), 91.5 \rightarrow 93.0 \% \mathrm{~B}$, $30 \min , \mathrm{RT}): t_{\mathrm{R}}=21.1 \mathrm{~min}$.

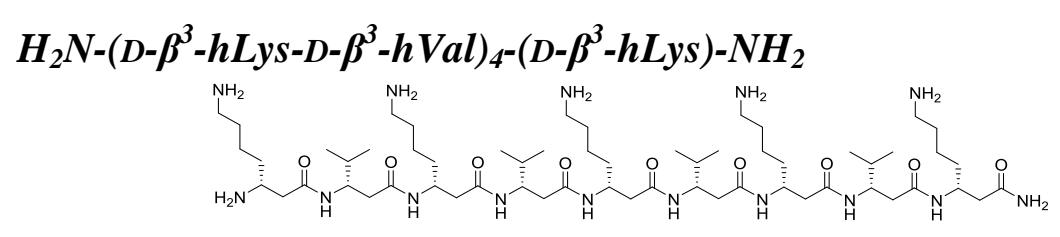

Pep 12

$\mathrm{C}_{59} \mathrm{H}_{117} \mathrm{~N}_{15} \mathrm{O}_{9}$

$1179.92 \mathrm{~g} / \mathrm{mol}$

The peptide Pep 12 was synthesised following SOP 5 using the amino acids described in Chapter 6.5.1.

MS (ESI, MeOH): m/z = $1180.9[\mathrm{M}+\mathrm{H}]^{+}, 591.0[\mathrm{M}+2 \mathrm{H}]^{2+}, 394.3[\mathrm{M}+3 \mathrm{H}]^{3+}$. 
HR-MS (ESI, MeOH):

$\begin{array}{lll}\mathrm{C}_{59} \mathrm{H}_{118} \mathrm{~N}_{15} \mathrm{O}_{9}[\mathrm{M}+\mathrm{H}]^{+} & \text {calculated: 1180:9231 } & \text { measured: 1180:9225; } \\ \mathrm{C}_{59} \mathrm{H}_{117} \mathrm{~N}_{15} \mathrm{O}_{9} \mathrm{Na}[\mathrm{M}+\mathrm{Na}]^{+} & \text {calculated: } 1202.9051 & \text { measured: 1202.9043; } \\ \mathrm{C}_{59} \mathrm{H}_{119} \mathrm{~N}_{15} \mathrm{O}_{9}[\mathrm{M}+2 \mathrm{H}]^{2+} & \text { calculated: } 590.9652 & \text { measured: } 590.9639 .\end{array}$

HPLC (preparative, A $\left(\mathrm{H}_{2} \mathrm{O}+0.1 \%\right.$ TFA $), \mathrm{B}\left(80 \% \mathrm{ACN}+20 \% \mathrm{H}_{2} \mathrm{O}+0.1 \%\right.$ TFA), $0.0 \rightarrow 60.0 \% \mathrm{~B}, 30 \mathrm{~min}, \mathrm{RT}): t_{\mathrm{R}}=19.4 \mathrm{~min}$.

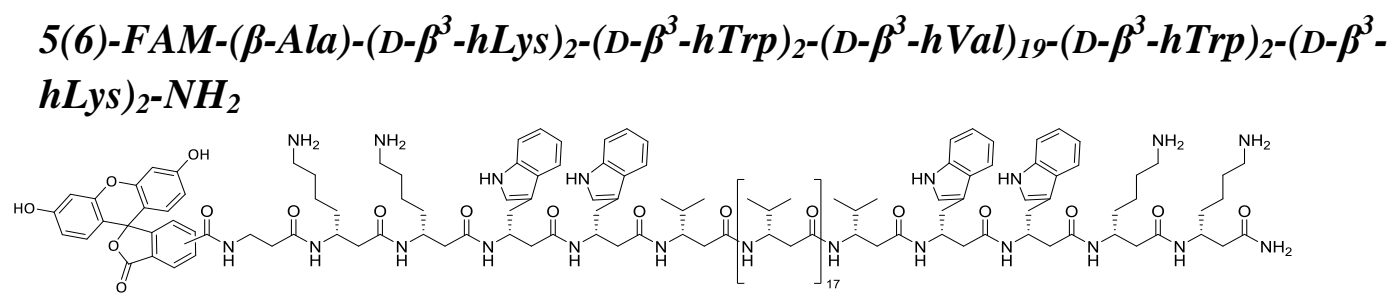

\section{Pep 13}

$$
\begin{aligned}
& \mathrm{C}_{214} \mathrm{H}_{331} \mathrm{~N}_{37} \mathrm{O}_{34} \\
& 3963.53 \mathrm{~g} / \mathrm{mol}
\end{aligned}
$$

The peptide Pep 13 was synthesised following SOP 6 using the amino acids described in Chapter 6.5.1. The fluorophore labelling was carried out in compliance with SOP 9.

MS (ESI, MeOH): $\mathrm{m} / \mathrm{z}=1983.8[\mathrm{M}+2 \mathrm{H}]^{2+}, 1322.9[\mathrm{M}+3 \mathrm{H}]^{3+}, 992.4[\mathrm{M}+4 \mathrm{H}]^{4+}, 794.1$ $[\mathrm{M}+5 \mathrm{H}]^{5+}, 661.9[\mathrm{M}+6 \mathrm{H}]^{6+}$.

\section{HR-MS (ESI, MeOH):}

$\begin{array}{lll}\mathrm{C}_{214} \mathrm{H}_{333} \mathrm{~N}_{37} \mathrm{O}_{34}[\mathrm{M}+2 \mathrm{H}]^{2+} & \text { calculated: } 1983.7758 & \text { measured: 1983.7778; } \\ \mathrm{C}_{214} \mathrm{H}_{334} \mathrm{~N}_{37} \mathrm{O}_{34}[\mathrm{M}+3 \mathrm{H}]^{3+} & \text { calculated: } 1322.8529 & \text { measured: 1322.8549; } \\ \mathrm{C}_{214} \mathrm{H}_{335} \mathrm{~N}_{37} \mathrm{O}_{34}[\mathrm{M}+4 \mathrm{H}]^{4+} & \text { calculated: } 992.3915 & \text { measured: 992.3928; } \\ \mathrm{C}_{214} \mathrm{H}_{336} \mathrm{~N}_{37} \mathrm{O}_{34}[\mathrm{M}+5 \mathrm{H}]^{5+} & \text { calculated: 794.1147 } & \text { measured: 794.1161; } \\ \mathrm{C}_{214} \mathrm{H}_{337} \mathrm{~N}_{37} \mathrm{O}_{34}[\mathrm{M}+6 \mathrm{H}]^{6+} & \text { calculated: } 661.9301 & \text { measured: 661.9309. }\end{array}$

HPLC (semi-preparative, A $\left(\mathrm{H}_{2} \mathrm{O}+0.1 \%\right.$ TFA $+0.1 \%$ HFIP $), \quad B \quad(\mathrm{MeOH}+0.1 \%$ $\mathrm{TFA}+0.1 \% \mathrm{HFIP}$ ), probe dissolved in $100 \% \mathrm{HFIP}, 88.0 \rightarrow 100.0 \% \mathrm{~B}, 30 \mathrm{~min}, 60{ }^{\circ} \mathrm{C}$ ): $t_{\mathrm{R}}=12.0 \mathrm{~min}$. 


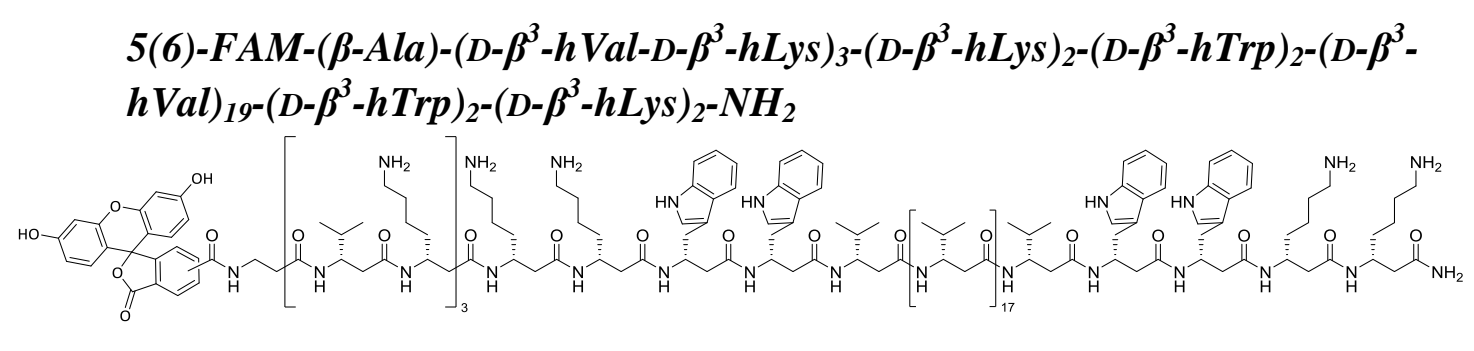

Pep 14

$\mathrm{C}_{253} \mathrm{H}_{406} \mathrm{~N}_{46} \mathrm{O}_{40}$

$4729.12 \mathrm{~g} / \mathrm{mol}$

The peptide Pep 14 was synthesised following SOP 6 using the amino acids described in Chapter 6.5.1. The fluorophore labelling was carried out in compliance with SOP 9.

MS (ESI, MeOH): $\mathrm{m} / \mathrm{z}=1578.0[\mathrm{M}+3 \mathrm{H}]^{3+}, 1184.0[\mathrm{M}+4 \mathrm{H}]^{4+}, 947.4[\mathrm{M}+5 \mathrm{H}]^{5+}, 789.6$ $[\mathrm{M}+6 \mathrm{H}]^{6+}, 677.0[\mathrm{M}+7 \mathrm{H}]^{7+}, 592.5[\mathrm{M}+8 \mathrm{H}]^{8+}$.

\section{HR-MS (ESI, MeOH):}

$\begin{array}{lll}\mathrm{C}_{25}{ }_{3} \mathrm{H}_{409} \mathrm{~N}_{46} \mathrm{O}_{40}[\mathrm{M}+3 \mathrm{H}]^{3+} & \text { calculated: } 1578.0476 & \text { measured: 1578.0492; } \\ \mathrm{C}_{253} \mathrm{H}_{410} \mathrm{~N}_{46} \mathrm{O}_{40}[\mathrm{M}+4 \mathrm{H}]^{4+} & \text { calculated: } 1183.7875 & \text { measured: 1183.7887; } \\ \mathrm{C}_{253} \mathrm{H}_{411} \mathrm{~N}_{46} \mathrm{O}_{40}[\mathrm{M}+5 \mathrm{H}]^{5+} & \text { calculated: } 947.2315 & \text { measured: } 947.2325 ; \\ \mathrm{C}_{253} \mathrm{H}_{412} \mathrm{~N}_{46} \mathrm{O}_{40}[\mathrm{M}+6 \mathrm{H}]^{6+} & \text { calculated: } 789.5274 & \text { measured: 789.5277; } \\ \mathrm{C}_{253} \mathrm{H}_{413} \mathrm{~N}_{46} \mathrm{O}_{40}[\mathrm{M}+7 \mathrm{H}]^{7+} & \text { calculated: } 677.0251 & \text { measured: 677.0253; } \\ \mathrm{C}_{253} \mathrm{H}_{414} \mathrm{~N}_{46} \mathrm{O}_{40}[\mathrm{M}+8 \mathrm{H}]^{8+} & \text { calculated: } 592.5229 & \text { measured: 592.5230. }\end{array}$

HPLC (semi-preparative, A $\left(\mathrm{H}_{2} \mathrm{O}+0.1 \%\right.$ TFA $+0.1 \%$ HFIP $), \quad B \quad(\mathrm{MeOH}+0.1 \%$ TFA+ $0.1 \%$ HFIP), probe dissolved in $100 \% \mathrm{HFIP}, 80.0 \rightarrow 100.0 \% \mathrm{~B}, 30 \mathrm{~min}, 60{ }^{\circ} \mathrm{C}$ ): $t_{\mathrm{R}}=16.6 \mathrm{~min}$.

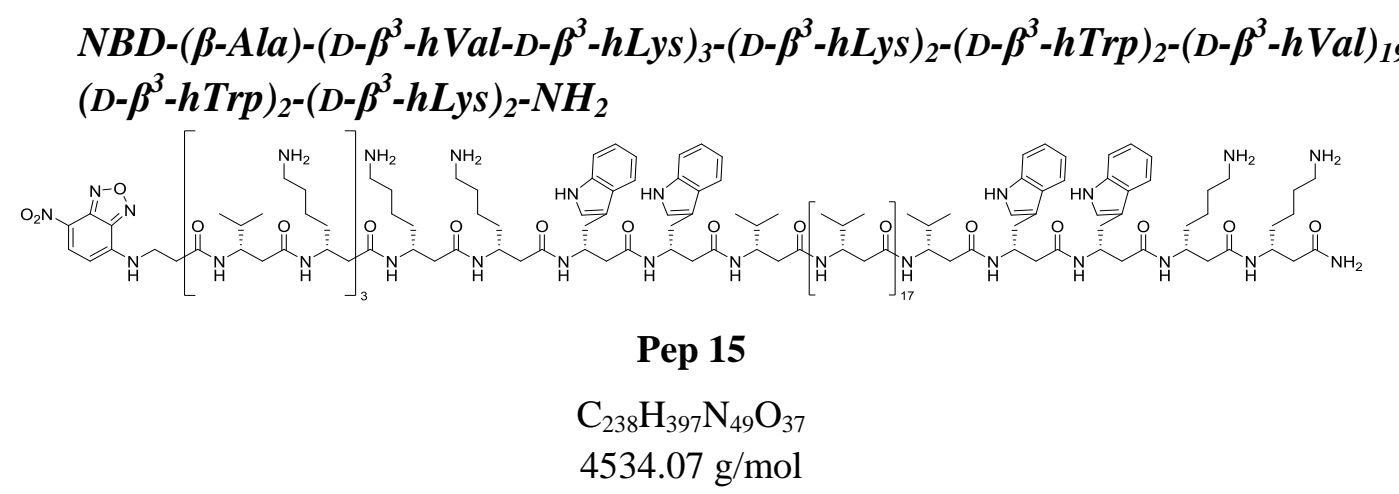

The peptide Pep 15 was synthesised following SOP 5 using the amino acids described in Chapter 6.5.1. The fluorophore labelling was performed with NBD-F (5.00 eq) in DMF 
and DIPEA (20.0 eq). This solution was given to the dry resin for gentle agitation overnight.

MS (ESI, MeOH): $\mathrm{m} / \mathrm{z}=1513.4[\mathrm{M}+3 \mathrm{H}]^{3+}, 1135.0[\mathrm{M}+4 \mathrm{H}]^{4+}, 908.2[\mathrm{M}+5 \mathrm{H}]^{5+}, 757.0$ $[\mathrm{M}+6 \mathrm{H}]^{6+}, 649.0[\mathrm{M}+7 \mathrm{H}]^{7+}$.

HR-MS (ESI, MeOH):

$\mathrm{C}_{238} \mathrm{H}_{400} \mathrm{~N}_{49} \mathrm{O}_{37}[\mathrm{M}+3 \mathrm{H}]^{3+} \quad$ calculated: $1513.0323 \quad$ measured: 1513.0319;

$\mathrm{C}_{238} \mathrm{H}_{401} \mathrm{~N}_{49} \mathrm{O}_{37}[\mathrm{M}+4 \mathrm{H}]^{4+} \quad$ calculated: $1135.0260 \quad$ measured: 1135.0261;

$\mathrm{C}_{238} \mathrm{H}_{402} \mathrm{~N}_{49} \mathrm{O}_{37}[\mathrm{M}+5 \mathrm{H}]^{5+} \quad$ calculated: $908.2223 \quad$ measured: 908.2227;

$\mathrm{C}_{238} \mathrm{H}_{403} \mathrm{~N}_{49} \mathrm{O}_{37}[\mathrm{M}+6 \mathrm{H}]^{6+} \quad$ calculated: $757.0198 \quad$ measured: 757.0199;

$\mathrm{C}_{238} \mathrm{H}_{404} \mathrm{~N}_{49} \mathrm{O}_{37}[\mathrm{M}+7 \mathrm{H}]^{7+} \quad$ calculated: $649.0180 \quad$ measured: 649.0176.

HPLC (preparative, A $\left(\mathrm{H}_{2} \mathrm{O}+0.1 \%\right.$ TFA $), \mathrm{B}(\mathrm{MeOH}+0.1 \%$ TFA $), 88.0 \rightarrow 98.0 \% \mathrm{~B}$, $30 \min , \mathrm{RT}): t_{\mathrm{R}}=18.9 \mathrm{~min}$.

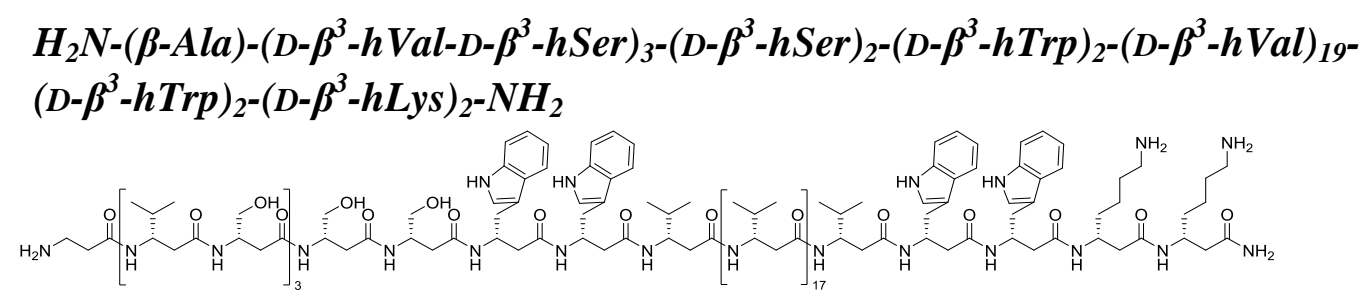

\section{Pep 16}

$\mathrm{C}_{217} \mathrm{H}_{361} \mathrm{~N}_{41} \mathrm{O}_{39}$ $4165.75 \mathrm{~g} / \mathrm{mol}$

The peptide Pep 16 was synthesised following SOP 6 using the amino acids described in Chapter 6.5.1. The fluorophore labelling was carried out in compliance with SOP 9.

MS (ESI, $\mathrm{MeOH}): \mathrm{m} / \mathrm{z}=1390.1[\mathrm{M}+3 \mathrm{H}]^{3+}$.

HR-MS (ESI, MeOH):

$\begin{array}{lll}\mathrm{C}_{217} \mathrm{H}_{364} \mathrm{~N}_{41} \mathrm{O}_{39}[\mathrm{M}+3 \mathrm{H}]^{3+} & \text { calculated: } 1390.2601 & \text { measured: 1390.2641; } \\ \mathrm{C}_{217} \mathrm{H}_{365} \mathrm{~N}_{41} \mathrm{O}_{39}[\mathrm{M}+4 \mathrm{H}]^{4+} & \text { calculated: } 1042.9469 & \text { measured: } 1042.9496 .\end{array}$




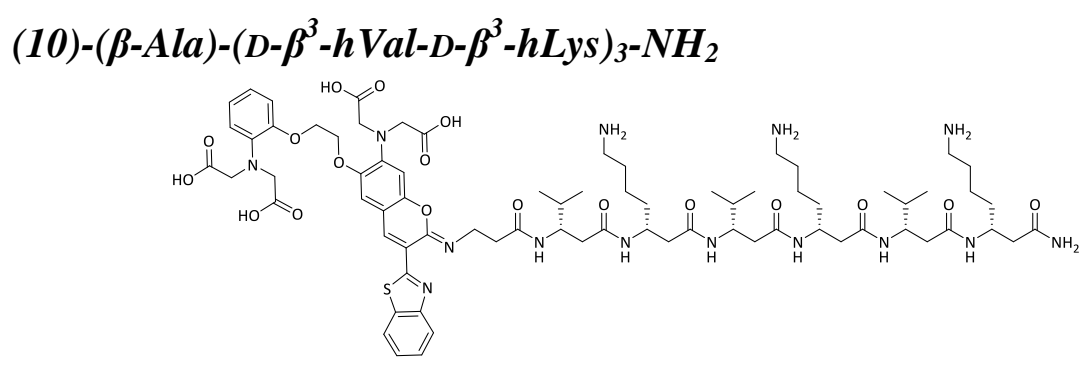

\section{Pep 18 \\ $\mathrm{C}_{74} \mathrm{H}_{108} \mathrm{~N}_{14} \mathrm{O}_{18} \mathrm{~S}$ \\ $1512.77 \mathrm{~g} / \mathrm{mol}$}

The peptide Pep 18 was synthesised following SOP 6 using the amino acids described in Chapter 6.5.1. The fluorophore labelling was carried out in compliance with SOP 10.

MS (ESI, MeOH): m/z = $757.4[\mathrm{M}+2 \mathrm{H}]^{2+}, 505.3[\mathrm{M}+3 \mathrm{H}]^{3+}$.

HR-MS (ESI, MeOH):

$\mathrm{C}_{74} \mathrm{H}_{110} \mathrm{~N}_{14} \mathrm{O}_{18} \mathrm{~S}[\mathrm{M}+2 \mathrm{H}]^{2+}$ calculated: $757.3916 \quad$ measured: 757.3917;

$\mathrm{C}_{74} \mathrm{H}_{111} \mathrm{~N}_{14} \mathrm{O}_{18} \mathrm{~S}[\mathrm{M}+3 \mathrm{H}]^{3+}$ calculated: 505.2635 measured: 505.2639.

HPLC (analytic, A $\left(\mathrm{H}_{2} \mathrm{O}+0.1 \%\right.$ TFA), B $(\mathrm{MeOH}+0.1 \%$ TFA $), 30.0 \rightarrow 100.0 \% \mathrm{~B}$, $\left.30 \min , 25^{\circ} \mathrm{C}\right): t_{\mathrm{R}}=15.9 \mathrm{~min}$.

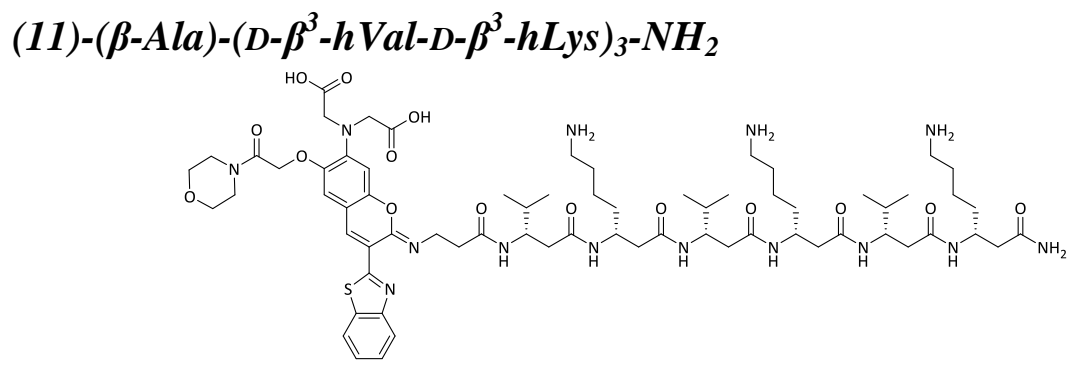

Pep 19

$\mathrm{C}_{68} \mathrm{H}_{104} \mathrm{~N}_{14} \mathrm{O}_{15} \mathrm{~S}$

$1388.75 \mathrm{~g} / \mathrm{mol}$

The peptide Pep 19 was synthesised following SOP 6 using the amino acids described in Chapter 6.5.1. The fluorophore labelling was carried out in compliance with SOP 10.

MS (ESI, MeOH): m/z = $695.4[\mathrm{M}+2 \mathrm{H}]^{2+}, 463.9[\mathrm{M}+3 \mathrm{H}]^{3+}$. 
HR-MS (ESI, MeOH):

$\begin{array}{lll}\mathrm{C}_{68} \mathrm{H}_{106} \mathrm{~N}_{14} \mathrm{O}_{15} \mathrm{~S}[\mathrm{M}+2 \mathrm{H}]^{2+} & \text { calculated: 695.3836 } & \text { measured: 695.3843; } \\ \mathrm{C}_{68} \mathrm{H}_{107} \mathrm{~N}_{14} \mathrm{O}_{15} \mathrm{~S}[\mathrm{M}+3 \mathrm{H}]^{3+} & \text { calculated: 463.9248 } & \text { measured: 463.9254. }\end{array}$

HPLC (analytic, A $\left(\mathrm{H}_{2} \mathrm{O}+0.1 \%\right.$ TFA), B (MeOH + 0.1\% TFA), 30.0 $\rightarrow 100.0 \% \mathrm{~B}$, $\left.30 \min , 25^{\circ} \mathrm{C}\right): t_{\mathrm{R}}=14.2 \mathrm{~min}$.

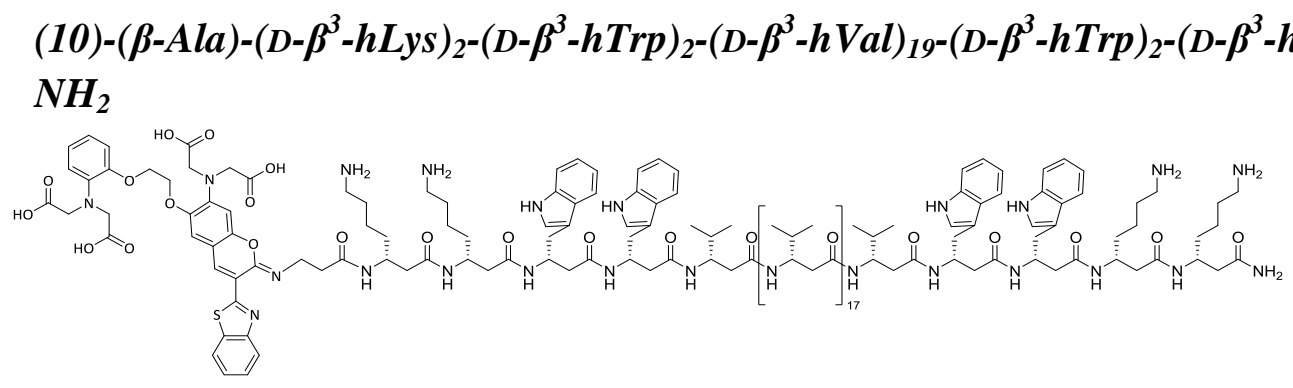

Pep 20

$\mathrm{C}_{225} \mathrm{H}_{346} \mathrm{~N}_{40} \mathrm{O}_{39} \mathrm{~S}$

$4264.60 \mathrm{~g} / \mathrm{mol}$

The peptide Pep 20 was synthesised following SOP 6 using the amino acids described in Chapter 6.5.1. The fluorophore labelling was carried out in compliance with SOP 10.

MS (ESI, MeOH): m/z = 2134.8 $[\mathrm{M}+2 \mathrm{H}]^{2+}, 1423.2[\mathrm{M}+3 \mathrm{H}]^{3+}, 1067.66[\mathrm{M}+4 \mathrm{H}]^{4+}, 854.33$ $[\mathrm{M}+5 \mathrm{H}]^{5+}$.

HR-MS (ESI, MeOH):

$\mathrm{C}_{225} \mathrm{H}_{349} \mathrm{~N}_{40} \mathrm{O}_{39} \mathrm{~S}[\mathrm{M}+3 \mathrm{H}]^{3+}$ calculated: 1423.2106

measured: 1423.2104;

$\mathrm{C}_{225} \mathrm{H}_{350} \mathrm{~N}_{40} \mathrm{O}_{39} \mathrm{~S}[\mathrm{M}+4 \mathrm{H}]^{4+}$ calculated: 1067.6598

measured: 1067.6598;

$\mathrm{C}_{225} \mathrm{H}_{351} \mathrm{~N}_{40} \mathrm{O}_{39} \mathrm{~S}[\mathrm{M}+5 \mathrm{H}]^{5+}$ calculated: 854.3293

measured: 854.3290 .

HPLC (preparative, A $\left(\mathrm{H}_{2} \mathrm{O}+0.1 \% \mathrm{TFA}+0.1 \% \mathrm{HFIP}\right), \mathrm{B}(\mathrm{MeOH}+0.1 \% \mathrm{TFA}+0.1 \%$ HFIP), probe freshly dissolved in $100 \%$ HFIP, $90.0 \rightarrow 100.0 \% \mathrm{~B}, 30 \mathrm{~min}, \mathrm{RT}$ ): $t_{\mathrm{R}}=14.6 \mathrm{~min}$. 


\section{Appendix}

\section{Appendix A Automatic microwave-supported SPPS}

Method 1 (synthesis scale $0.15 \mathrm{mmol}$ )

\begin{tabular}{|llll|} 
Reagents & $\mathbf{0 . 1 5} \mathbf{~ m m o l}$ & Reagents & $\mathbf{0 . 1 5} \mathbf{~ m m o l}$ \\
\hline Wash solvent & DMF & Activator 1 & $0.5 \mathrm{M} \mathrm{DIC} \mathrm{in} \mathrm{DMF}$ \\
\hline $\begin{array}{l}\text { Deprotection } \\
\text { Cocktail }\end{array}$ & $\begin{array}{l}20 \% \text { Piperidine } \\
\text { in DMF }\end{array}$ & Activator Base 1 & $\begin{array}{l}1.0 \mathrm{M} \text { Oxyma in DMF + } \\
0.1 \mathrm{M} \text { DIPEA }\end{array}$ \\
\hline Amino Acids & $0.2 \mathrm{M} \mathrm{in} \mathrm{DMF}$ & Activator 2 & $0.5 \mathrm{M} / \mathrm{HATU}$ and \\
& & & $0.5 \mathrm{M} / \mathrm{HOAT}$ in DMF \\
\hline Capping 1 & $1.0 \mathrm{M} \mathrm{Ac}_{2} \mathrm{O}$ in DMF & Activator Base 2 & $2 \mathrm{M}$ DIPEA in NMP \\
\hline Capping 2 & $0.25 \mathrm{M}$ Oxyma in DMF + & & \\
& $0.03 \mathrm{M}$ DIPEA & & \\
\hline
\end{tabular}

Amino acid cycles

\begin{tabular}{|c|c|c|c|}
\hline & Parameters & Microwave method & Bubble time \\
\hline Deprotection & Deprotection Volume: 8 & $\begin{array}{l}70^{\circ} \mathrm{C}, 140 \mathrm{~W}, 180 \mathrm{~s}, \\
\Delta \mathrm{T}=2^{\circ} \mathrm{C}\end{array}$ & $\begin{array}{l}\text { On }=2 \mathrm{~s} \\
\text { Off }=3 \mathrm{~s}\end{array}$ \\
\hline 4xWash & Volume 6, Drain Time: 20 & & \\
\hline Wash & Volume 6, Drain Time: 25 & & \\
\hline Deprotection & Deprotection Volume: 8 & $\begin{array}{l}70^{\circ} \mathrm{C}, 140 \mathrm{~W}, 180 \mathrm{~s}, \\
\Delta \mathrm{T}=2{ }^{\circ} \mathrm{C}\end{array}$ & $\begin{array}{l}\text { On }=2 \mathrm{~s} \\
\text { Off }=3 \mathrm{~s}\end{array}$ \\
\hline $5 \times$ Wash & Volume 6, Drain Time: 20 & & \\
\hline Wash & Volume 6, Drain Time: 25 & & \\
\hline Coupling & $\begin{array}{l}\text { Amino Acid Volume: } 3.75 \\
\text { Activator } 1 \text { Volume: } 1.5 \\
\text { Activator Base } 1 \text { Volume: } 0.75\end{array}$ & $\begin{array}{l}\text { 1. } 25^{\circ} \mathrm{C}, 0 \mathrm{~W}, 60 \mathrm{~s}, \\
\Delta \mathrm{T}=2^{\circ} \mathrm{C} \\
\text { 2. } 75^{\circ} \mathrm{C}, 190 \mathrm{~W}, 15 \mathrm{~s}, \\
\Delta \mathrm{T}=2^{\circ} \mathrm{C} \\
\text { 3. } 90^{\circ} \mathrm{C}, 350 \mathrm{~W}, 285 \mathrm{~s}, \\
\Delta \mathrm{T}=2^{\circ} \mathrm{C} \\
4.25^{\circ} \mathrm{C}, 0 \mathrm{~W}, 60 \mathrm{~s}, \\
\Delta \mathrm{T}=2^{\circ} \mathrm{C}\end{array}$ & $\begin{array}{l}\text { On }=2 s \\
\text { Off }=3 s\end{array}$ \\
\hline $3 \times$ Wash & Volume 6, Drain Time: 20 & & \\
\hline Wash & Volume 6, Drain Time: 25 & & \\
\hline Coupling & $\begin{array}{l}\text { Amino Acid Volume: } 3.75 \\
\text { Activator } 2 \text { Volume: } 1.5 \\
\text { Activator Base } 2 \text { Volume: } 0.75\end{array}$ & $\begin{array}{l}\text { 1. } 25^{\circ} \mathrm{C}, 0 \mathrm{~W}, 60 \mathrm{~s}, \\
\Delta \mathrm{T}=2^{\circ} \mathrm{C} \\
\text { 2. } 75^{\circ} \mathrm{C}, 43 \mathrm{~W}, 1140 \mathrm{~s}, \\
\Delta \mathrm{T}=2{ }^{\circ} \mathrm{C}\end{array}$ & $\begin{array}{l}\text { On }=2 \mathrm{~s} \\
\text { Off }=3 \mathrm{~s}\end{array}$ \\
\hline $3 \times$ Wash & Volume 6, Drain Time: 20 & & \\
\hline Wash & Volume 6, Drain Time: 25 & & \\
\hline Capping & $\begin{array}{l}\text { Capping } 1 \text { Volume: } 5 \\
\text { Capping } 2 \text { Volume: } 5\end{array}$ & $\begin{array}{l}\text { 1. } 65^{\circ} \mathrm{C}, 65 \mathrm{~W}, 30 \mathrm{~s}, \\
\Delta \mathrm{T}=5^{\circ} \mathrm{C} \\
\text { 2. } 65^{\circ} \mathrm{C}, 0 \mathrm{~W}, 30 \mathrm{~s}, \\
\Delta \mathrm{T}=5^{\circ} \mathrm{C} \\
\text { 3. } 65^{\circ} \mathrm{C}, 65 \mathrm{~W}, 30 \mathrm{~s}, \\
\Delta \mathrm{T}=5^{\circ} \mathrm{C} \\
4.65^{\circ} \mathrm{C}, 0 \mathrm{~W}, 30 \mathrm{~s}, \\
\Delta \mathrm{T}=5^{\circ} \mathrm{C}\end{array}$ & $\begin{array}{l}\text { On }=1 \mathrm{~s} \\
\text { Off }=3 \mathrm{~s}\end{array}$ \\
\hline $\begin{array}{l}\text { 2×Wash Thru } \\
\text { Mainfold }\end{array}$ & Volume 6, Drain Time: 20 & & \\
\hline
\end{tabular}




\begin{tabular}{|ll|}
\hline $\begin{array}{l}\text { Wash Thru } \\
\text { Mainfold }\end{array}$ & Volume 6, Drain Time: 25 \\
\hline $\mathbf{2} \times$ Wash & Volume 6, Drain Time: 20 \\
\hline $\mathbf{2} \times$ Wash & Volume 6, Drain Time: 25 \\
\hline
\end{tabular}

Method 2 (synthesis scale $0.1 \mathrm{mmol}$ )

\begin{tabular}{|ll|}
\hline Reagents & $\mathbf{0 . 1} \mathrm{mmol}$ \\
\hline Wash solvent & $\mathrm{DMF}$ \\
\hline $\begin{array}{l}\text { Deprotection } \\
\text { Cocktail }\end{array}$ & $20 \%$ Piperidine in DMF \\
\hline Amino Acids & $0.2 \mathrm{M}$ in DMF \\
\hline Activator 1 & $0.5 \mathrm{M} \mathrm{DIC} \mathrm{in} \mathrm{DMF}$ \\
\hline Activator Base 1 & $\begin{array}{l}1.0 \mathrm{M} \text { Oxyma in DMF + } \\
0.1 \mathrm{M} \mathrm{DIPEA}\end{array}$ \\
\hline Capping 1 & $1.0 \mathrm{M} \mathrm{Ac} 2 \mathrm{O}$ in DMF \\
\hline Capping 2 & $0.25 \mathrm{M} \mathrm{Oxyma} \mathrm{in} \mathrm{DMF} \mathrm{+}$ \\
\hline
\end{tabular}

Amino acid cycles (additional steps for the first amino acid in italic letters)

\begin{tabular}{|c|c|c|c|}
\hline & Parameters & Microwave method & Bubble time \\
\hline Deprotection & Deprotection Volume: 5 & $\begin{array}{l}\text { 1. } 75^{\circ} \mathrm{C}, 260 \mathrm{~W}, 15 \mathrm{~s}, \\
\Delta \mathrm{T}=2^{\circ} \mathrm{C} \\
\text { 2. } 90^{\circ} \mathrm{C}, 60 \mathrm{~W}, 50 \mathrm{~s}, \\
\Delta \mathrm{T}=1^{\circ} \mathrm{C}\end{array}$ & $\begin{array}{l}\text { On }=2 \mathrm{~s} \\
\text { Off }=3 \mathrm{~s}\end{array}$ \\
\hline Wash & Volume 6, Drain Time: 10 & & \\
\hline Deprotection & Deprotection Volume: & $\begin{array}{l}\text { 1. } 75^{\circ} \mathrm{C}, 260 \mathrm{~W}, 15 \mathrm{~s}, \\
\Delta \mathrm{T}=2^{\circ} \mathrm{C} \\
\text { 2. } 90^{\circ} \mathrm{C}, 60 \mathrm{~W}, 50 \mathrm{~s}, \\
\Delta \mathrm{T}=1^{\circ} \mathrm{C}\end{array}$ & $\begin{array}{l}\text { On }=2 \mathrm{~s} \\
\text { Off }=3 \mathrm{~s}\end{array}$ \\
\hline Wash & Volume 6, Drain Time: 10 & & \\
\hline $3 \times$ Wash & Volume 6, Drain Time: 5 & & \\
\hline Wash & Volume 6, Drain Time: 10 & & \\
\hline Coupling & $\begin{array}{l}\text { Amino Acid Volume: } 2.5 \\
\text { Activator } 1 \text { Volume: } 2.0 \\
\text { Activator Base } 1 \text { Volume: } 0.5\end{array}$ & $\begin{array}{l}\text { 1. } 85^{\circ} \mathrm{C}, 300 \mathrm{~W}, 20 \mathrm{~s}, \\
\Delta \mathrm{T}=1^{\circ} \mathrm{C} \\
\text { 2. } 105^{\circ} \mathrm{C}, 100 \mathrm{~W}, 135 \mathrm{~s}, \\
\Delta \mathrm{T}=1{ }^{\circ} \mathrm{C}\end{array}$ & $\begin{array}{l}\text { On }=5 \mathrm{~s} \\
\text { Off }=2 \mathrm{~s}\end{array}$ \\
\hline Wash & Volume 6, Drain Time: 10 & & \\
\hline $3 \times$ Wash & Volume 6, Drain Time: 5 & & \\
\hline Wash & Volume 6, Drain Time: 10 & & \\
\hline Coupling & $\begin{array}{l}\text { Amino Acid Volume: } 2.5 \\
\text { Activator } 1 \text { Volume: } 2.0 \\
\text { Activator Base } 1 \text { Volume: } 0.5\end{array}$ & $\begin{array}{l}\text { 1. } 85^{\circ} \mathrm{C}, 300 \mathrm{~W}, 20 \mathrm{~s}, \\
\Delta T=1{ }^{\circ} \mathrm{C} \\
\text { 2. } 105^{\circ} \mathrm{C}, 100 \mathrm{~W}, 135 \mathrm{~s}, \\
\Delta T=1{ }^{\circ} \mathrm{C}\end{array}$ & $\begin{array}{l}O n=5 s \\
\text { Off }=2 s\end{array}$ \\
\hline Wash & Volume 6, Drain Time: 5 & & \\
\hline Wash & Volume 6, Drain Time: 10 & & \\
\hline Capping & $\begin{array}{l}\text { Capping } 1 \text { Volume: } 2.5 \\
\text { Capping } 2 \text { Volume: } 2.5\end{array}$ & $\begin{array}{l}\text { 1. } 65^{\circ} \mathrm{C}, 65 \mathrm{~W}, 30 \mathrm{~s}, \\
\Delta \mathrm{T}=5^{\circ} \mathrm{C} \\
\text { 2. } 65^{\circ} \mathrm{C}, 0 \mathrm{~W}, 30 \mathrm{~s}, \\
\Delta \mathrm{T}=5^{\circ} \mathrm{C}\end{array}$ & $\begin{array}{l}\text { On }=5 \mathrm{~s} \\
\text { Off }=2 \mathrm{~s}\end{array}$ \\
\hline
\end{tabular}




\begin{tabular}{|c|c|}
\hline & $\begin{array}{l}\text { 3. } 65^{\circ} \mathrm{C}, 65 \mathrm{~W}, 30 \mathrm{~s}, \\
\Delta \mathrm{T}=5^{\circ} \mathrm{C} \\
4.65^{\circ} \mathrm{C}, 0 \mathrm{~W}, 30 \mathrm{~s}, \\
\Delta \mathrm{T}=5^{\circ} \mathrm{C}\end{array}$ \\
\hline $\begin{array}{l}\text { Wash Thru } \\
\text { Mainfold }\end{array}$ & Volume 6, Drain Time: 10 \\
\hline $\begin{array}{l}2 \times \text { Wash Thru } \\
\text { Mainfold }\end{array}$ & Volume 6, Drain Time: 5 \\
\hline Wash & Volume 6, Drain Time: 5 \\
\hline Wash & Volume 6, Drain Time: 10 \\
\hline
\end{tabular}

\section{Appendix B Lipids [https://avantilipids.com 16.10.2018 17:40]}

\section{1,2-Dimyristoyl-sn-glycero-3-phosphocholine (DMPC)}

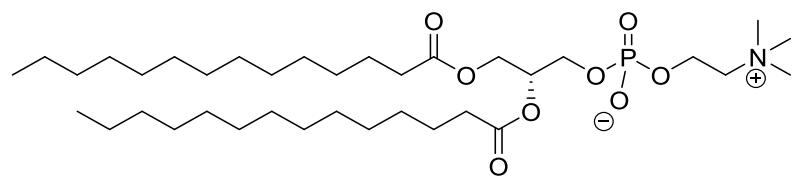

Molecular Weight [g/mol]:

677.933

Molecular Formula:

$\mathrm{C}_{36} \mathrm{H}_{72} \mathrm{NO}_{8} \mathrm{P}$

Saturation degree:

14:0/14:0

Transition Temp:

\section{1,2-Dipalmitoyl-sn-glycero-3-phosphocholine (DPPC)}

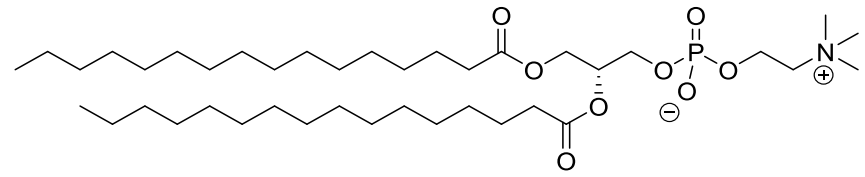

Molecular Weight [g/mol]:

733.039

Molecular Formula:

$\mathrm{C}_{40} \mathrm{H}_{80} \mathrm{NO}_{8} \mathrm{P}$

Saturation degree:

16:0/16:0

Transition Temp:

$41^{\circ} \mathrm{C}$

\section{1,2-Dioleoyl-sn-glycero-3-phosphocholine (DOPC)}<smiles>CCCCCCCCCCCCCCCCCCCCCCCCCC(=O)OCC(COP(=O)(O)OCC[N+](C)(C)C)OC(=O)CCCCCCCCCC</smiles>

Molecular Weight [g/mol]:

786.113

Molecular Formula: $\mathrm{C}_{44} \mathrm{H}_{84} \mathrm{NO}_{8} \mathrm{P}$

Saturation degree: 18:1/18:1

Transition Temp: $-17^{\circ} \mathrm{C}$ 
1,2-Dioleoyl-sn-glycero-3-phospho-(1'-rac-glycerol) (sodium salt) (DOPG)

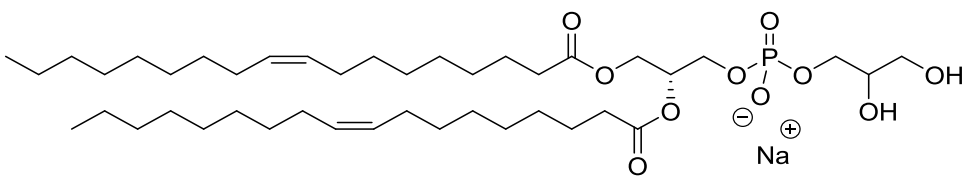

Molecular Weight [g/mol]:

797.026

Molecular Formula:

$\mathrm{C}_{42} \mathrm{H}_{78} \mathrm{NO}_{10} \mathrm{PNa}$

Saturation degree:

18:1/18:1

Transition Temp:

$-18^{\circ} \mathrm{C}$

1-Palmitoyl-2-stearoyl-(10-doxyl)-sn-glycero-3-phosphocholine (16:0-16 DOXYL PC)

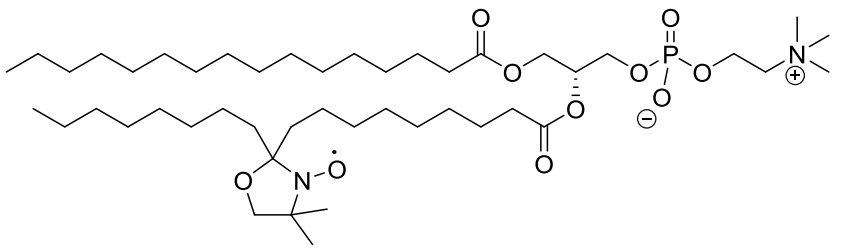

Molecular Weight [g/mol]:

862.188

Molecular Formula:

$\mathrm{C}_{46} \mathrm{H}_{90} \mathrm{~N} 2 \mathrm{O} 10 \mathrm{P}$.

Saturation degree:

16:0/18:0 (10 DOXYL)

1-Palmitoyl-2-stearoyl-(16-doxyl)-sn-glycero-3-phosphocholine (16:0-16 DOXYL PC)

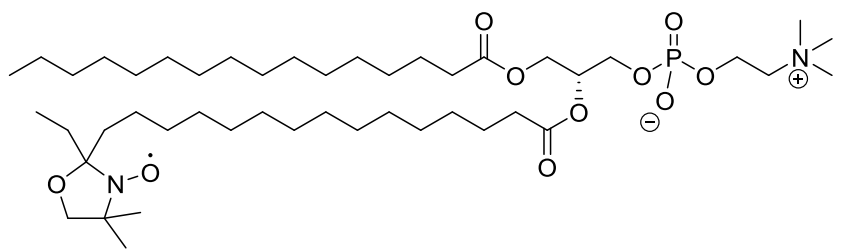

Molecular Weight [g/mol]:

862.188

Molecular Formula:

$\mathrm{C}_{46} \mathrm{H}_{90} \mathrm{~N} 2 \mathrm{O} 10 \mathrm{P}$.

Saturation degree:

16:0/18:0 (16 DOXYL)

\section{1,2-Dioleoyl-sn-glycero-3-phosphate (sodium salt) (DOPA)}

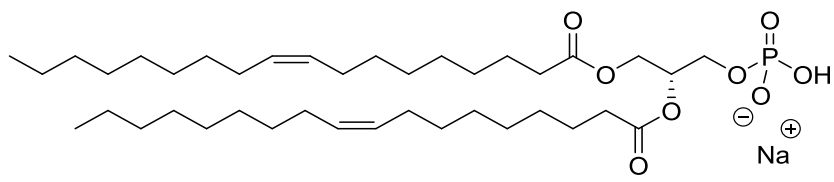

Molecular Weight [g/mol]:

722.948

Molecular Formula:

$\mathrm{C}_{39} \mathrm{H}_{72} \mathrm{NO}_{8} \mathrm{PNa}$

Saturation degree:

18:1/18:1 


\section{1,2-Dioleoyl-sn-glycero-3-phospho-L-serine (sodium salt) (DOPS)}

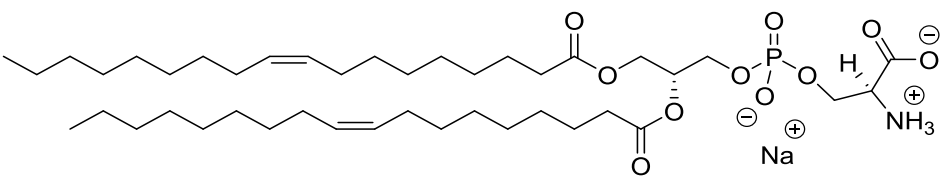

Molecular Weight [g/mol]:

810.025

Molecular Formula:

$\mathrm{C}_{42} \mathrm{H}_{77} \mathrm{NO}_{10} \mathrm{PNa}$

Saturation degree:

18:1/18:1

\section{Appendix C Spectroscopy of Peptides}

\section{Analytic Data of Pep 1}

a)

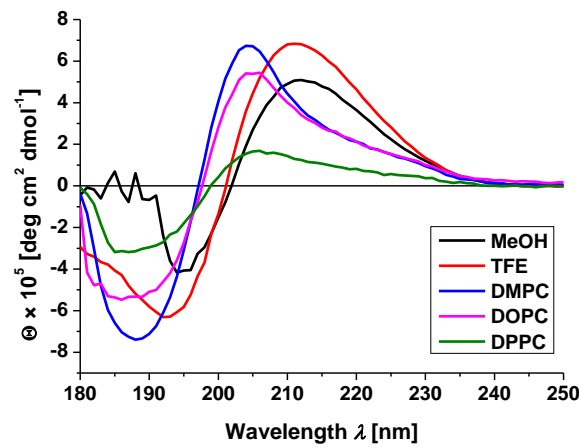

b)

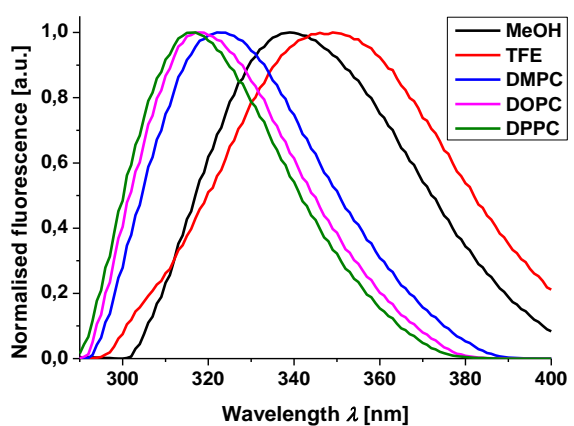

Figure 6.1 Analytic Data of Pep 1. a) Overlay of CD spectra of Pep 1 in organic solvents (MeOH, TFE) and in LUV (DMPC, DOPC, DPPC) show a characteristic right handed 14-helix. b) Fluorescence spectra of Pep 1 in organic solvents $\left(F_{\text {Max,MeOH }}=339 \mathrm{~nm}, F_{\text {Max,TFE }}=349 \mathrm{~nm}\right)$ and in $\operatorname{LUV}\left(F_{\text {Max,DMPC }}=322 \mathrm{~nm}, F_{\text {Max,DPPC }}=317 \mathrm{~nm}\right.$, $\left.\mathrm{F}_{\text {Max,DOPC }}=318 \mathrm{~nm}\right)$.

\section{Analytic Data of Pep 2}

a)

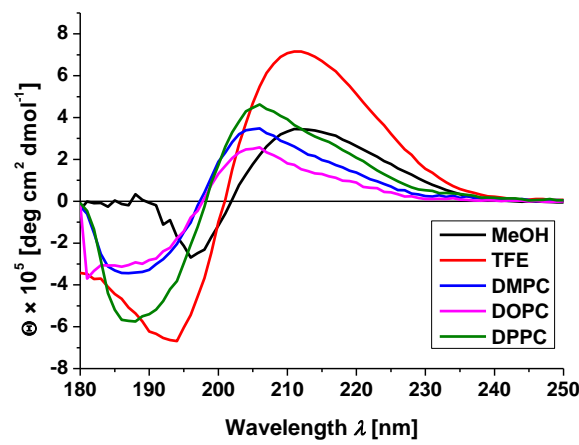

b)

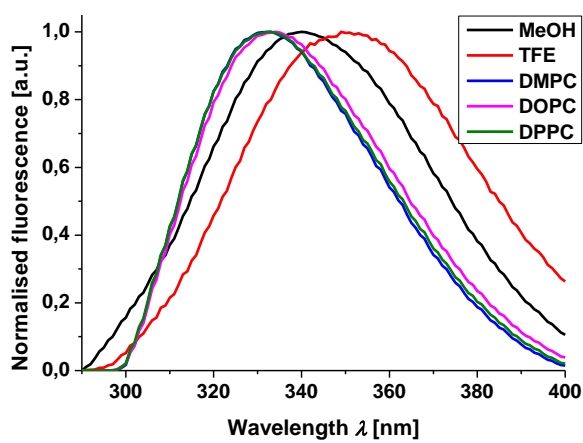

Figure 6.2 Analytic Data of Pep 2. a) Overlay of CD spectra of Pep 2 in organic solvents (MeOH, TFE) and in LUV (DMPC, DOPC, DPPC) show a characteristic right handed 14-helix. b) Fluorescence spectra of Pep 2 in organic solvents $\left(F_{\text {Max,MeOH }}=340 \mathrm{~nm}, F_{\text {Max,TFE }}=349 \mathrm{~nm}\right)$ and in $L U V\left(F_{\text {Max }, \text { DMPC }}=333 \mathrm{~nm}, F_{\text {Max }, \text { DPPC }}=333 \mathrm{~nm}\right.$, $\left.\mathrm{F}_{\text {Max }, \text { DOPC }}=334 \mathrm{~nm}\right)$. 


\section{Analytic Data of Pep 3}

a)

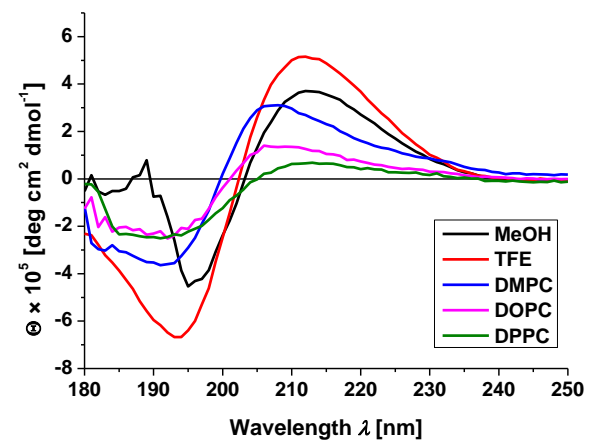

b)

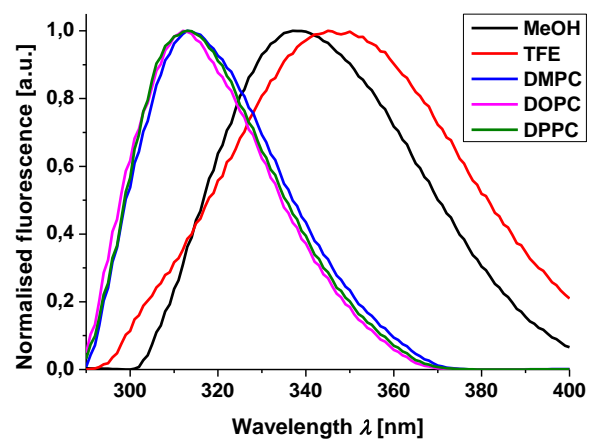

Figure 6.3 Analytic Data of Pep 3. a) Analytic Data of Pep 1. a) Overlay of CD spectra of Pep 3 in organic solvents (MeOH, TFE) and in LUV (DMPC, DOPC, DPPC) show a characteristic right handed 14-helix. b) Fluorescence spectra of Pep 3 in organic solvents $\left(F_{\text {Max,MeOH }}=337 \mathrm{~nm}, F_{\text {Max,TFE }}=345 \mathrm{~nm}\right)$ and in LUV $\left(F_{\text {Max,DMPC }}=313 \mathrm{~nm}\right.$, $\left.\mathrm{F}_{\text {Max }, \text { DPPC }}=313 \mathrm{~nm}, \mathrm{~F}_{\text {Max,DOPC }}=312 \mathrm{~nm}\right)$.

\section{Analytic Data of Pep 4}

a)

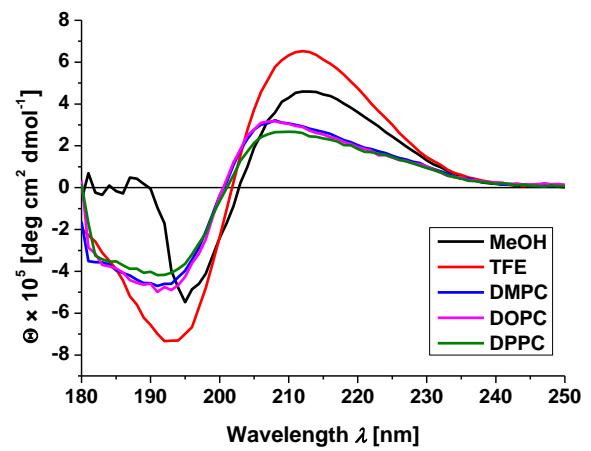

b)

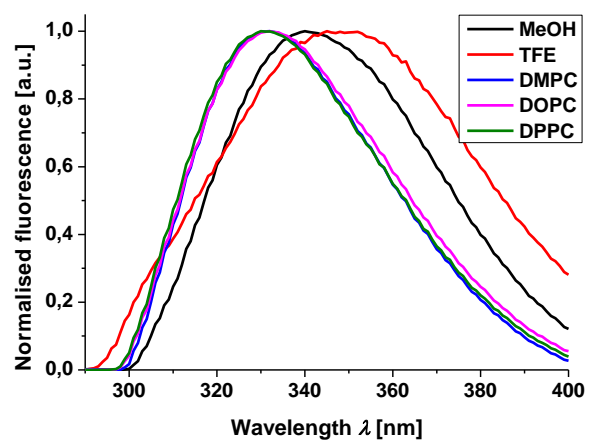

Figure 6.4 Analytic Data of Pep 4. a) Overlay of CD spectra of Pep 4 in organic solvents (MeOH, TFE) and in LUV (DMPC, DOPC, DPPC) show a characteristic right handed 14-helix. b) Fluorescence spectra of Pep 4 in organic solvents $\left(F_{\mathrm{Max}, \mathrm{MeOH}}=340 \mathrm{~nm}, \mathrm{~F}_{\mathrm{Max}, \mathrm{TFE}}=345 \mathrm{~nm}\right)$ and in $\mathrm{LUV}\left(\mathrm{F}_{\mathrm{Max}, \mathrm{DMPC}}=332 \mathrm{~nm}, \mathrm{~F}_{\mathrm{Max}, \mathrm{DPPC}}=330 \mathrm{~nm}\right.$, $\left.\mathrm{F}_{\text {Max }, \mathrm{DOPC}}=332 \mathrm{~nm}\right)$.

\section{Analytic Data of Pep 5}

a)

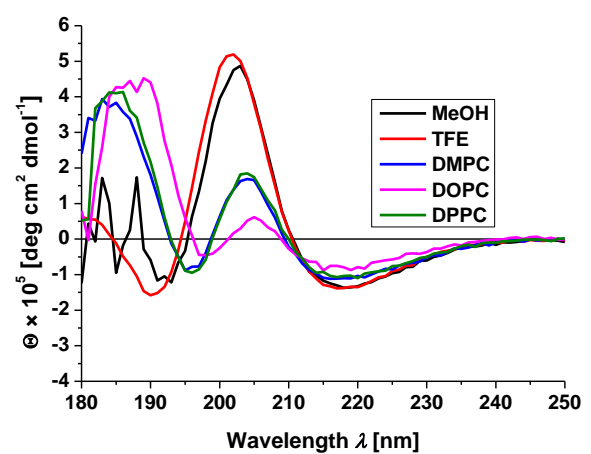

b)

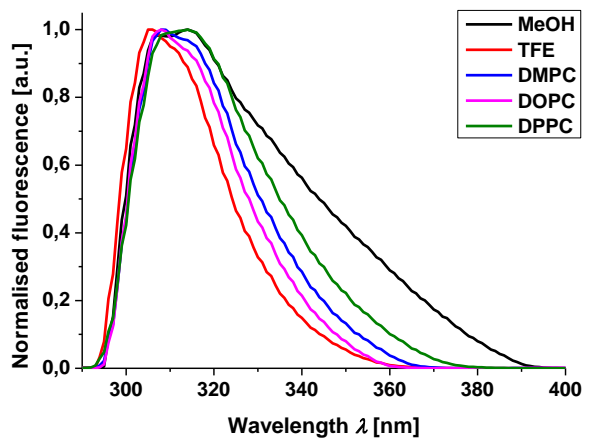

Figure 6.5 Analytic Data of Pep 5. a) Overlay of CD spectra of Pep 5 in organic solvents (MeOH, TFE) and in LUV (DMPC, DOPC, DPPC) show a characteristic left handed 12-helix. b) Fluorescence spectra of Pep 5 in organic solvents $\left(F_{\text {Max,MeOH }}=314 \mathrm{~nm}, F_{\text {Max,TFE }}=305 \mathrm{~nm}\right)$ and in $\operatorname{LUV}\left(F_{\text {Max }, \text { DMPC }}=308 \mathrm{~nm}, F_{\text {Max }, \text { DPPC }}=314 \mathrm{~nm}\right.$,

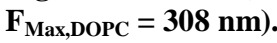




\section{Analytic Data of Pep 6}

a)

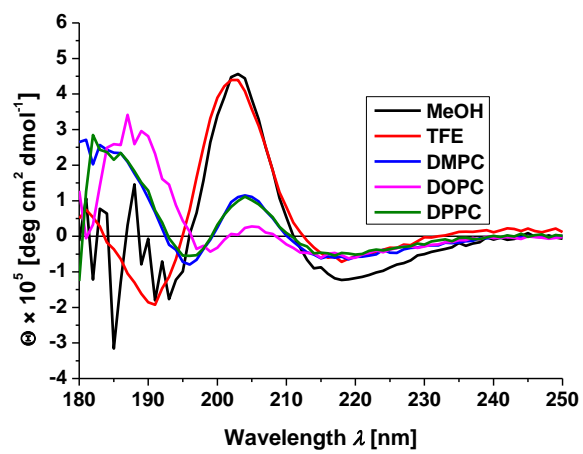

b)

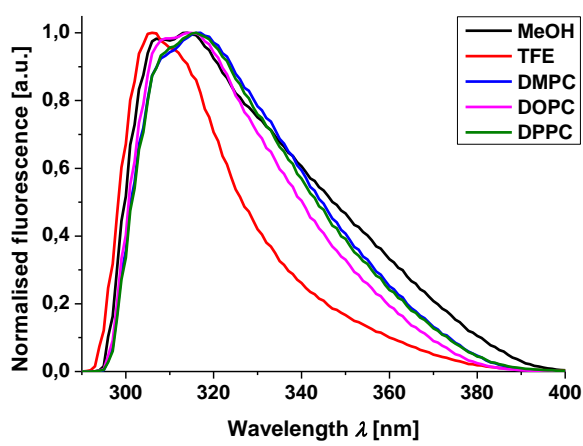

Figure 6.6 Analytic Data of Pep 6. a) Overlay of CD spectra of Pep 6 in organic solvents (MeOH, TFE) and in LUV (DMPC, DOPC, DPPC) show a characteristic left handed 12-helix. b) Fluorescence spectra of Pep 6 in organic solvents $\left(F_{\text {Max,MeOH }}=314 \mathrm{~nm}, F_{\text {Max,TFE }}=306 \mathrm{~nm}\right)$ and in LUV $\left(F_{\text {Max,DMPC }}=317 \mathrm{~nm}, F_{\text {Max }, \text { DPPC }}=3316 \mathrm{~nm}\right.$, $\left.\mathrm{F}_{\text {Max,DOPC }}=314 \mathrm{~nm}\right)$.

\section{Analytic Data of Pep 7}

a)

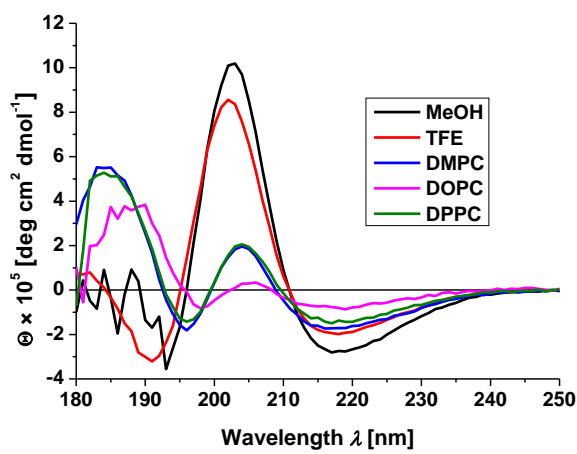

b)

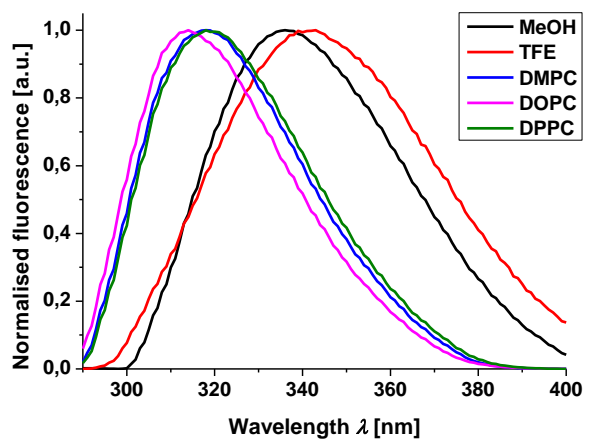

Figure 6.7 Analytic Data of Pep 7. a) Overlay of CD spectra of Pep 7 in organic solvents (MeOH, TFE) and in LUV (DMPC, DOPC, DPPC) show a characteristic left handed 12-helix. b) Fluorescence spectra of Pep 7 in organic solvents $\left(F_{\text {Max,MeOH }}=336 \mathrm{~nm}, F_{\text {Max }, \text { TFE }}=343 \mathrm{~nm}\right)$ and in $\operatorname{LUV}\left(F_{\text {Max,DMPC }}=318 \mathrm{~nm}\right.$, $F_{\text {Max,DPPC }}=318 \mathrm{~nm}$, $\left.\mathrm{F}_{\text {Max,DOPC }}=314 \mathrm{~nm}\right)$.

\section{Analytic Data of Pep 8}

a)

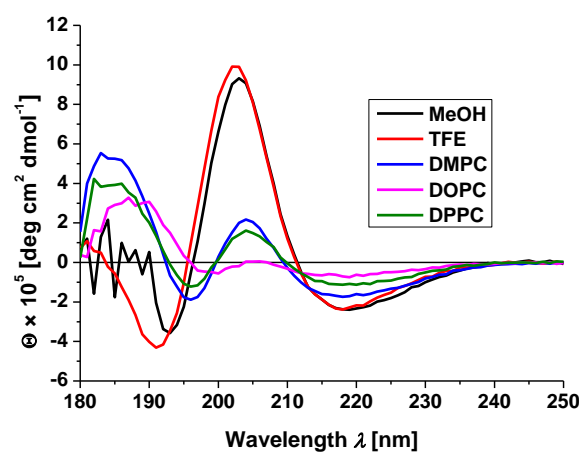

b)

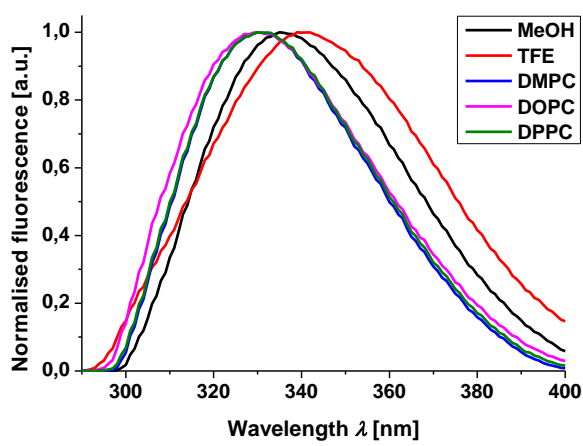

Figure 6.8 Analytic Data of Pep 8. a) Overlay of CD spectra of Pep 8 in organic solvents (MeOH, TFE) and in LUV (DMPC, DOPC, DPPC) show a characteristic left handed 12 helix. b) Fluorescence spectra of Pep 8 in organic solvents $\left(F_{\text {Max,MeOH }}=335 \mathrm{~nm}, F_{\text {Max }, \text { TFE }}=339 \mathrm{~nm}\right)$ and in $\operatorname{LUV}\left(F_{\text {Max,DMPC}}=330 \mathrm{~nm}, F_{\text {Max }, \text { DPPC }}=330 \mathrm{~nm}\right.$, $\left.\mathrm{F}_{\text {Max,DOPC }}=331 \mathrm{~nm}\right)$. 


\section{Analytic Data of Pep 9}

a)

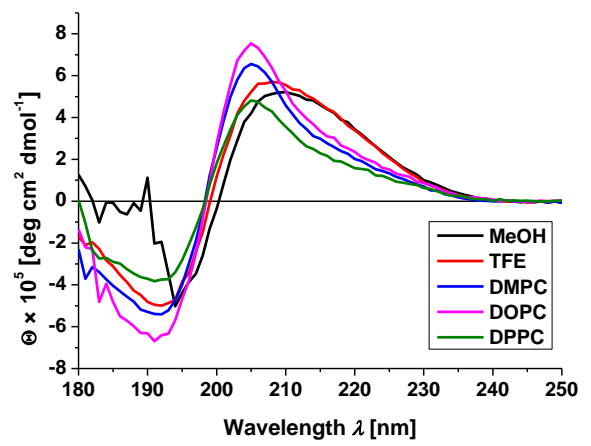

b)

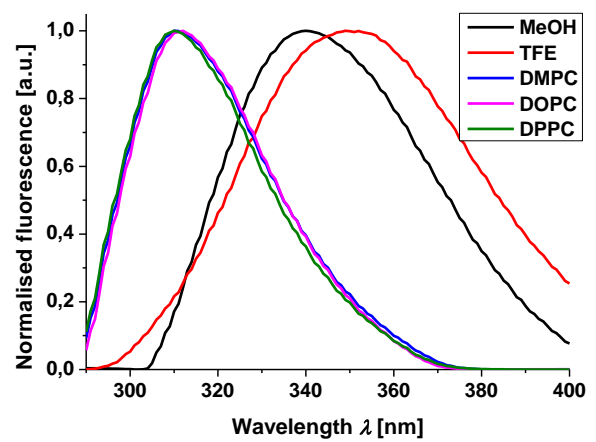

Figure 6.9 Analytic Data of Pep 9. a) Overlay of CD spectra of Pep 9 in organic solvents (MeOH, TFE) and in LUV (DMPC, DOPC, DPPC) show a characteristic right handed 14-helix. b) Fluorescence spectra of Pep 9 in organic solvents $\left(F_{\text {Max,MeOH }}=340 \mathrm{~nm}, F_{\text {Max,TFE }}=349 \mathrm{~nm}\right)$ and in $\operatorname{LUV}\left(F_{\text {Max }, \text { DMPC }}=310 \mathrm{~nm}, F_{\text {Max,DPPC }}=310 \mathrm{~nm}\right.$, $\mathbf{F}_{\text {Max,DOPC }}=312$ nm).

\section{Analytic Data of Pep 10}

a)

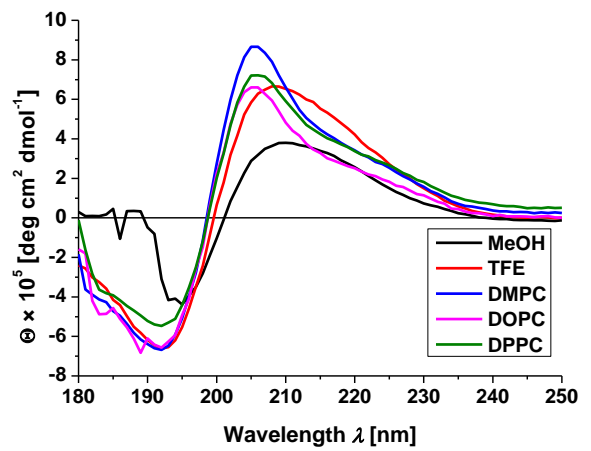

b)

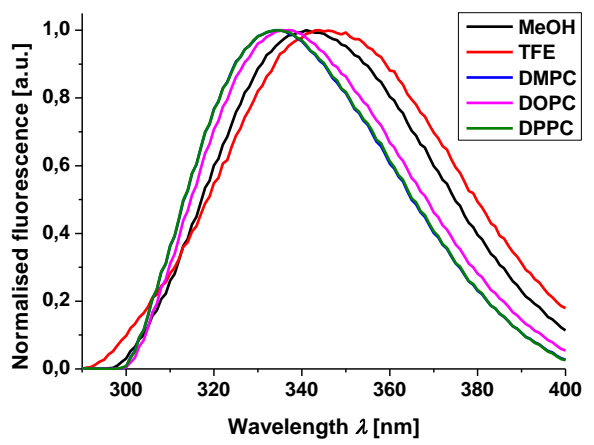

Figure 6.10 Analytic Data of Pep 10. a) Overlay of CD spectra of Pep 10 in organic solvents (MeOH, TFE) and in LUV (DMPC, DOPC, DPPC) show a characteristic right handed 14-helix. b) Fluorescence spectra of Pep 10 in organic solvents $\left(F_{\text {Max,MeOH }}=341 \mathrm{~nm}, F_{\text {Max,TFE }}=344 \mathrm{~nm}\right)$ and in LUV $\left(F_{\text {Max,DMPC }}=334 \mathrm{~nm}, F_{\text {Max,DPPC }}=335 \mathrm{~nm}\right.$, $\left.\mathbf{F}_{\text {Max,DOPC }}=337 \mathrm{~nm}\right)$.

\section{Analytic Data of Pep 11}

a)

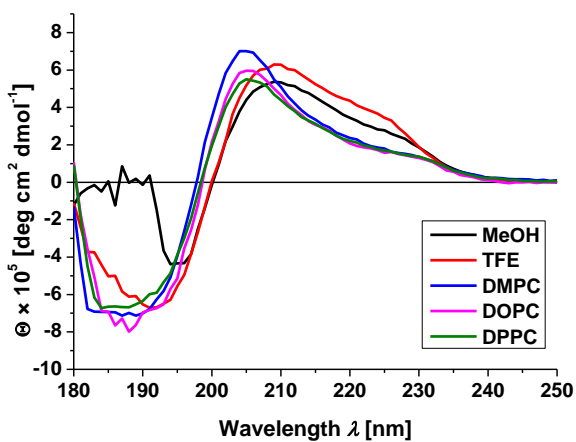

b)

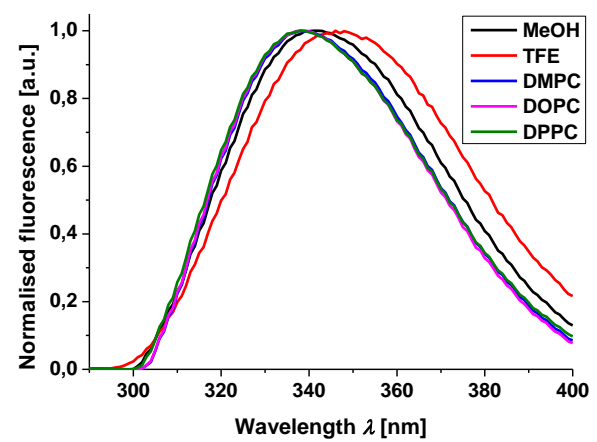

Figure 6.11 Analytic Data of Pep 11. a) Overlay of CD spectra of Pep 11 in organic solvents (MeOH, TFE) and in LUV (DMPC, DOPC, DPPC) show a characteristic right handed 14-helix. b) Fluorescence spectra of Pep 10 in organic solvents $\left(F_{\text {Max,MeOH }}=342 \mathrm{~nm}, F_{\text {Max,TFE }}=346 \mathrm{~nm}\right)$ and in $\operatorname{LUV}\left(F_{\text {Max,DMPC }}=338 \mathrm{~nm}, F_{\text {Max,DPC }}=338 \mathrm{~nm}\right.$, $\mathbf{F}_{\text {Max }, \text { DOPC }}=338$ nm). 
Analytic Data of Pep 12

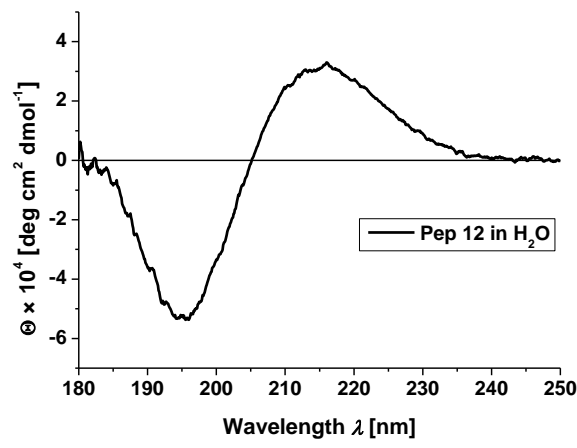

Figure 6.12 CD spectrum of Pep 12 in organic ultrapure water shows a characteristic right handed 14-helix.

\section{Analytic Data of Pep 18 and Pep 19}

a)

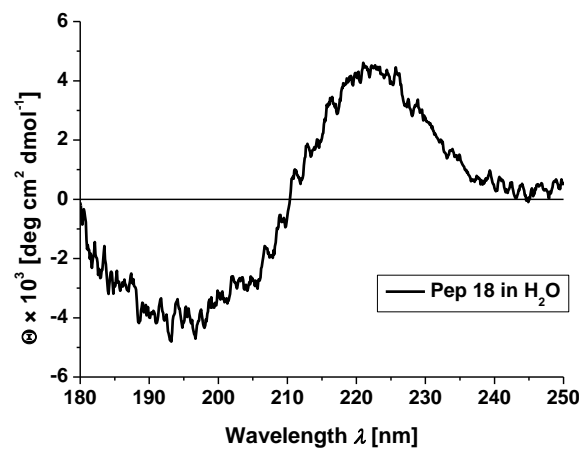

b)

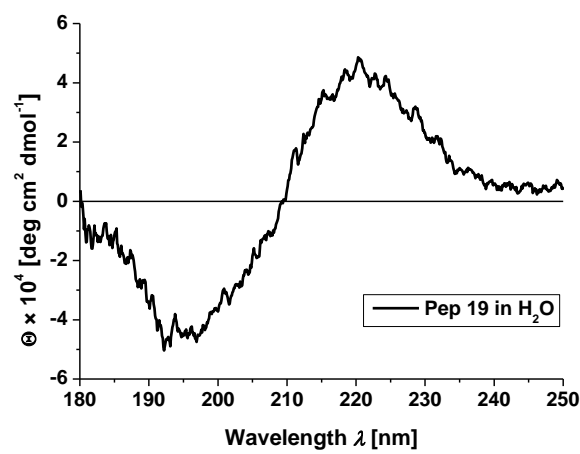

Figure 6.13 a) CD spectrum of Pep 12 in organic ultrapure water shows a characteristic right handed 14-helix and CD spectrum of Pep 12 in organic ultrapure water shows a characteristic right handed 14-helix.

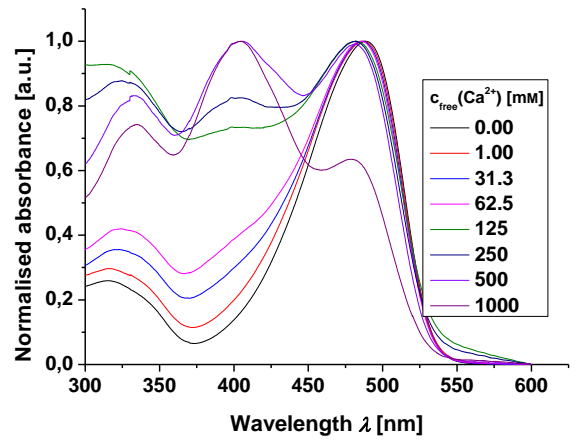

Figure 6.14 Absorbance spectra of Pep 19 were recorded during a $\mathrm{Ca}^{2+}$ titration. The normalised absorbance intensities were plotted against the wavelengths $\lambda$. 


\section{Paralax measurements}

a) Pep 1 in DOPC/10:0-10 DOXYL PC

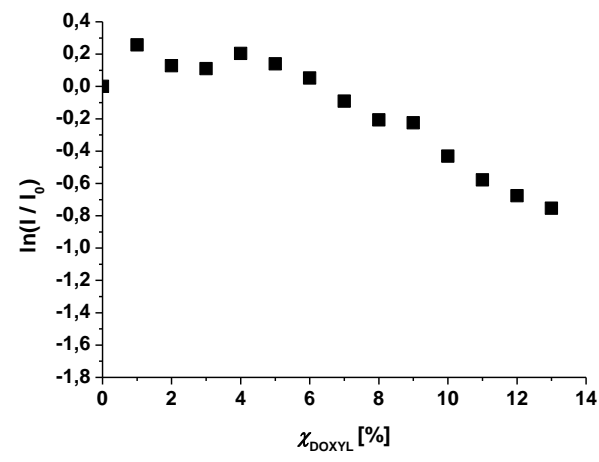

c) Pep 2 in DOPC/10:0-10 DOXYL PC

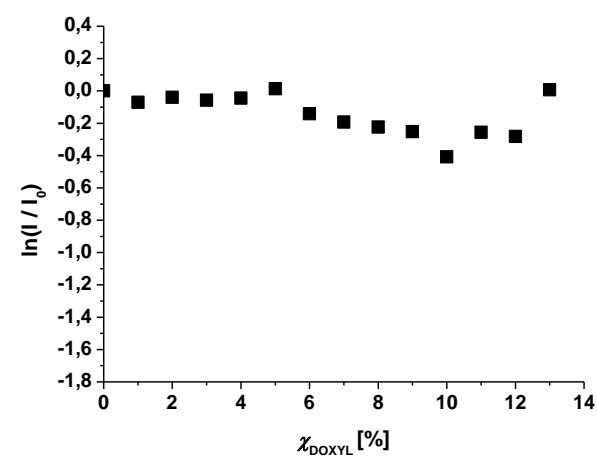

b) Pep 1 in DOPC/16:0-16 DOXYL PC

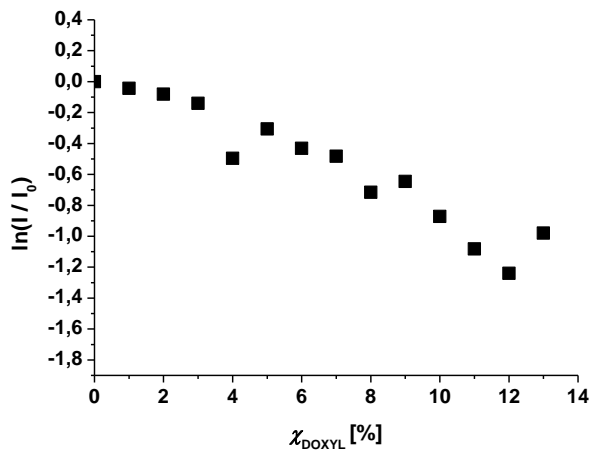

d) Pep 2 in DOPC/16:0-16 DOXYL PC

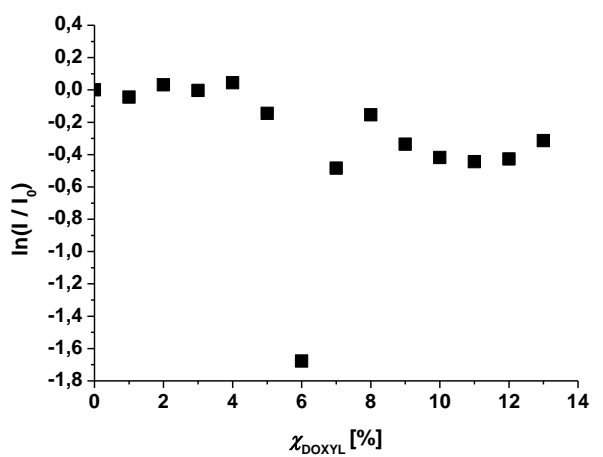

Figure 6.15 Fluorescence depth quenching curves of Pep 1 and Pep 2 with varying percentages of 10:0-10 DOXYL PC or 16:0-16 DOXYL PC derived from the corresponding fluorescence spectra.

Titration curve PBS

a) Pep 13 in DOPC

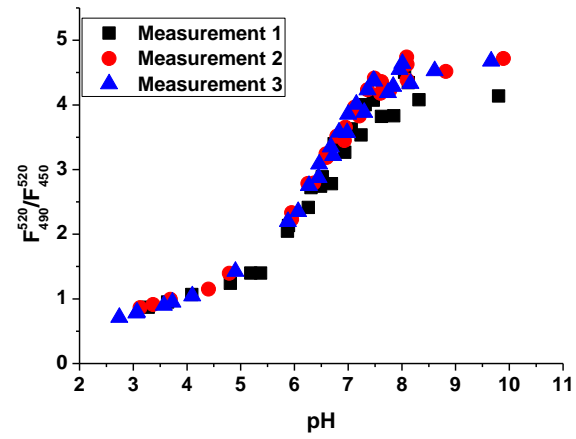

b) Pep 13 in DOPC/DOPG (50:50)

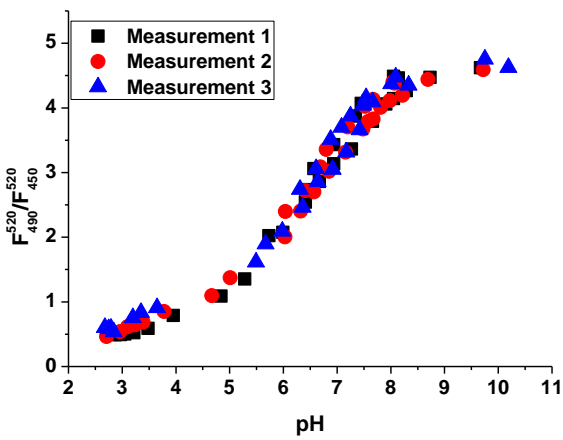


c) Pep 14 in DOPC

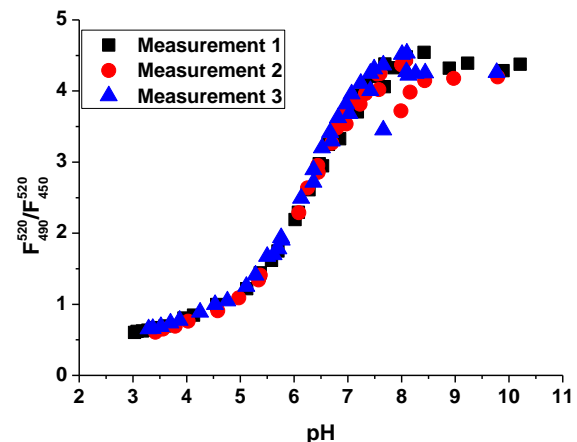

d) Pep 14 in DOPC/DOPG (50:50)

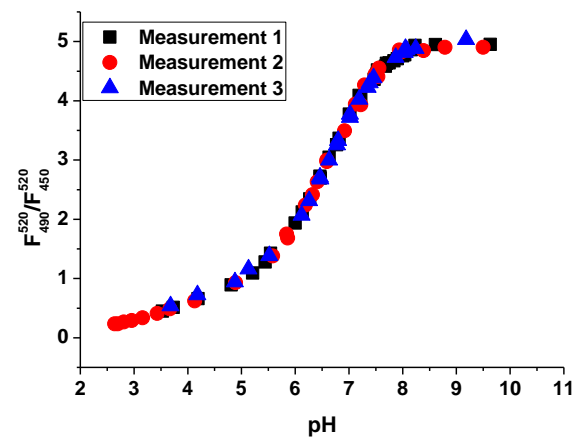

Figure 6.16 Titration curve of Pep 13 and Pep 14 in DOPC and DOPC/DOPG (50:50) vesicles rehydrated and extruded in PBS buffer.

\section{Titration curve HEPES}

a) Pep 13 in DOPC

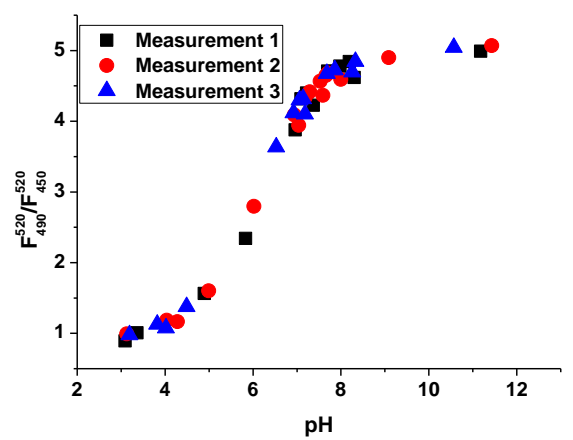

c) Pep 14 in DOPC

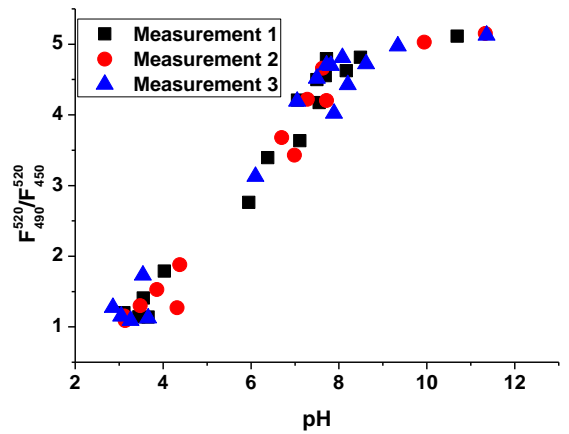

b) Pep 13 in DOPC/DOPA (90:10)

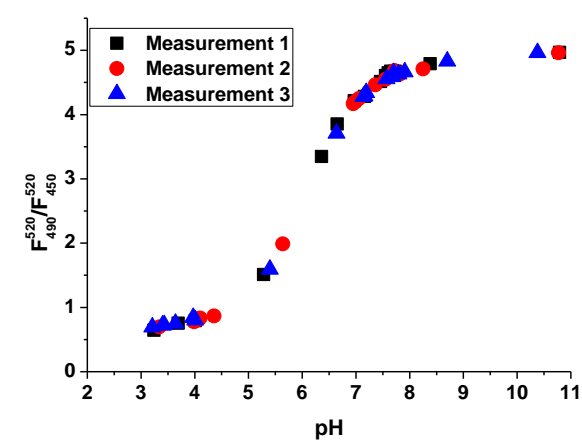

d) Pep 14 in DOPC/DOPA (90:10)

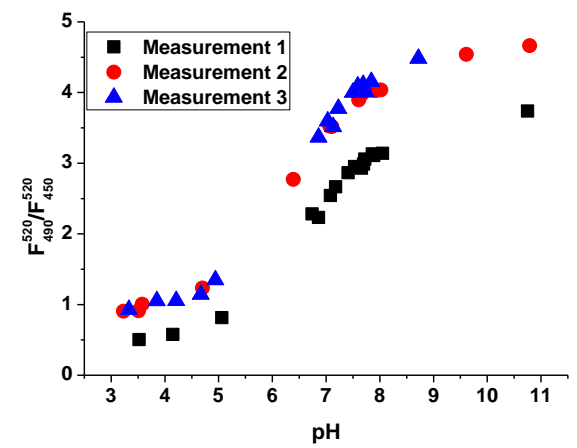

Figure 6.17 Titration curve of Pep 13 and Pep 14 in DOPC and DOPC/DOPA (90:10) vesicles rehydrated and extruded in HEPES buffer. 


\section{Size distribution}

$100 \mathrm{~nm}$

a) Pep 13 in DOPC

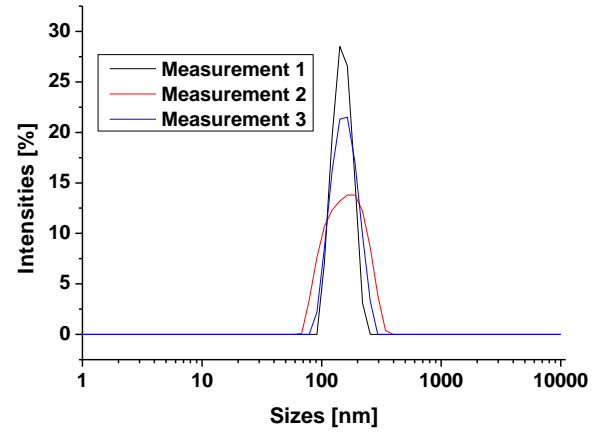

c) Pep 14 in DOPC

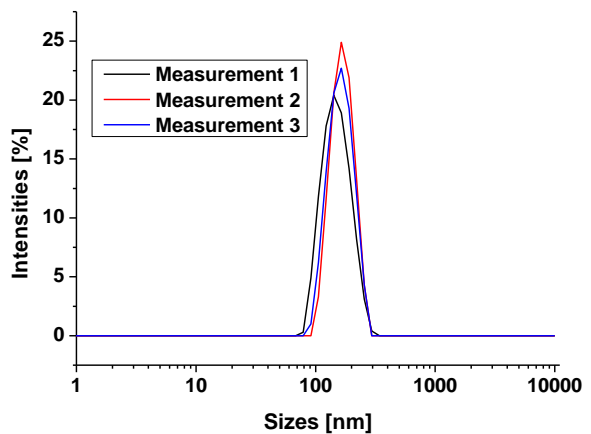

b) Pep 13 in DOPC/DOPG (50:50)

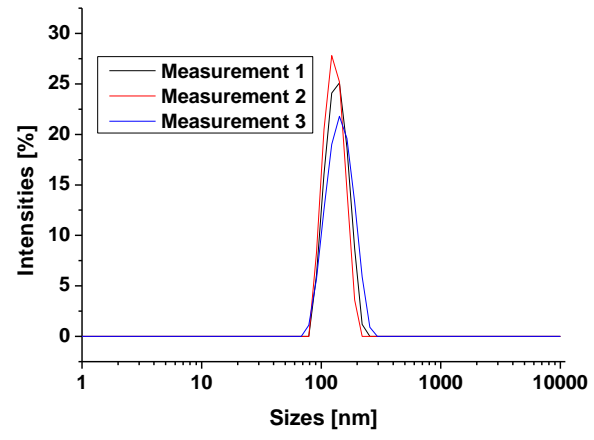

d) Pep 14 in DOPC/DOPG(50:50)

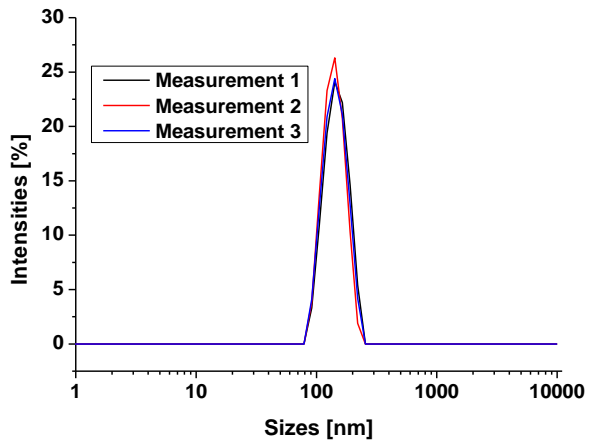

Figure 6.18 DLS measurements of Pep 13 and Pep 14 in DOPC and DOPC/DOPG (50:50) vesicles rehydrated and extruded in PBS buffer.

\section{$400 \mathrm{~nm}$}

a) Pep 13 in DOPC

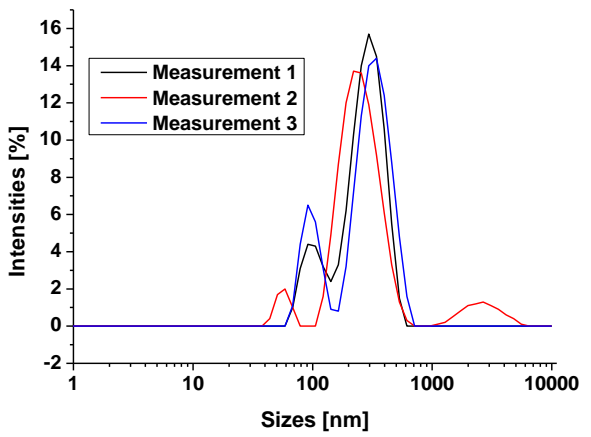

b) Pep 13 in DOPC/DOPA (90:10)

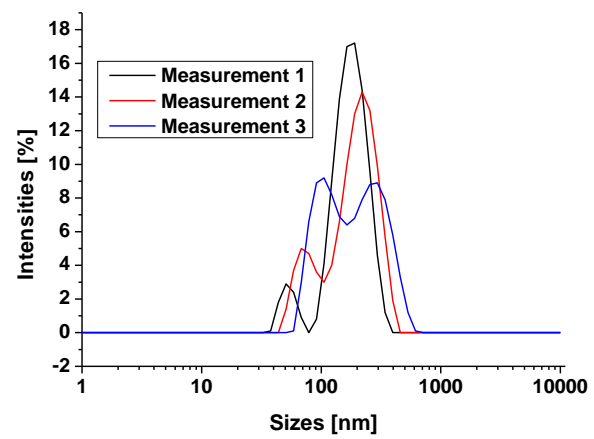


c) Pep 14 in DOPC

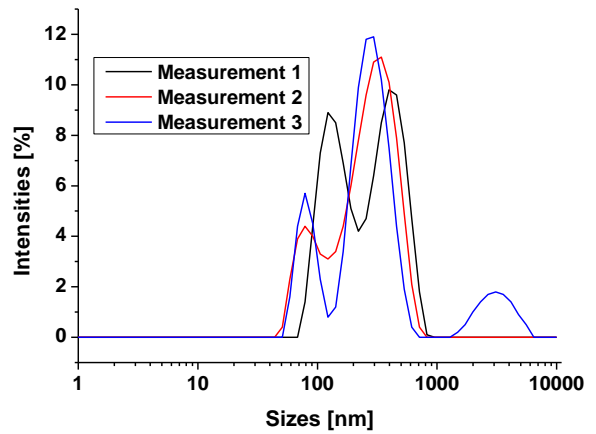

d) Pep 14 in DOPC/DOPA (90:10)

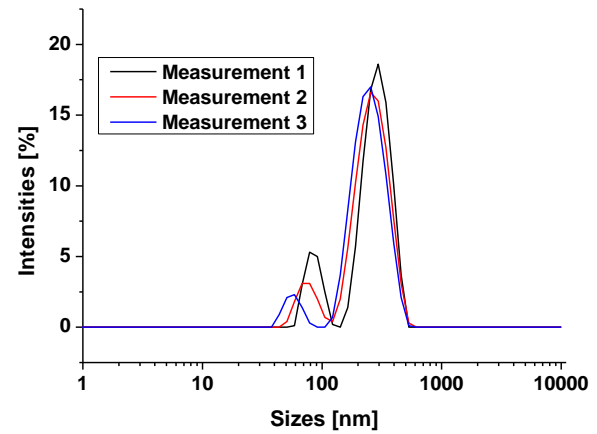

Figure 6.19 DLS measurements of Pep 13 and Pep 14 in DOPC and DOPC/DOPA (90:10) vesicles rehydrated and extruded in HEPES buffer.

Fluorescence measurements of Pep 13 and Pep 14 with multiplied pH steps

a) Pep 13 in DOPC
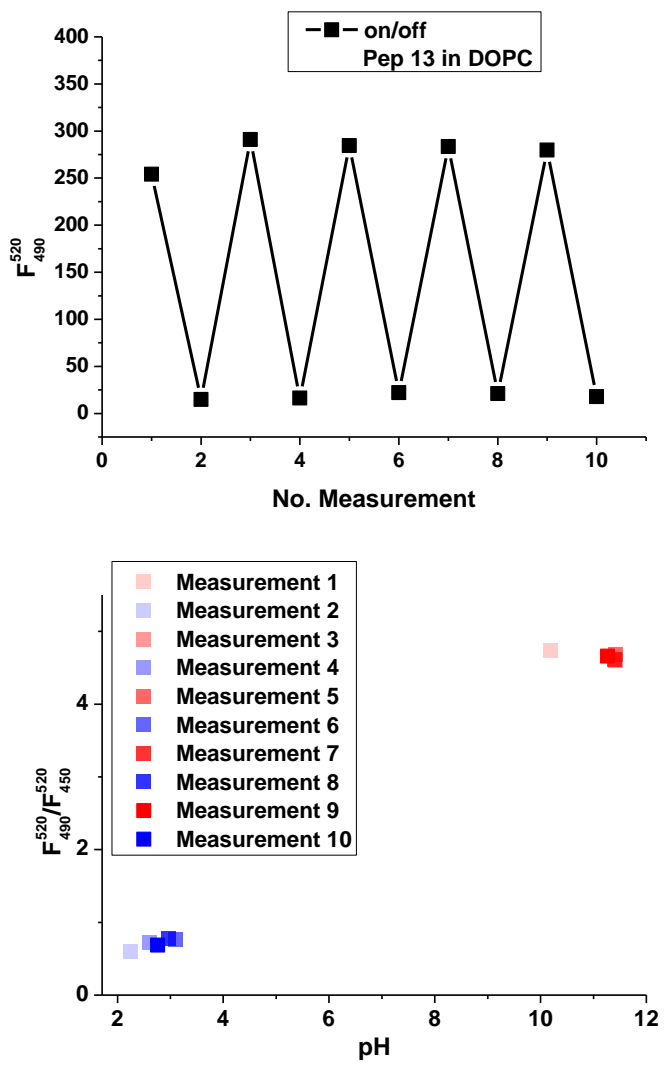

b) Pep 13 in DOPC/DOPG (50:50)
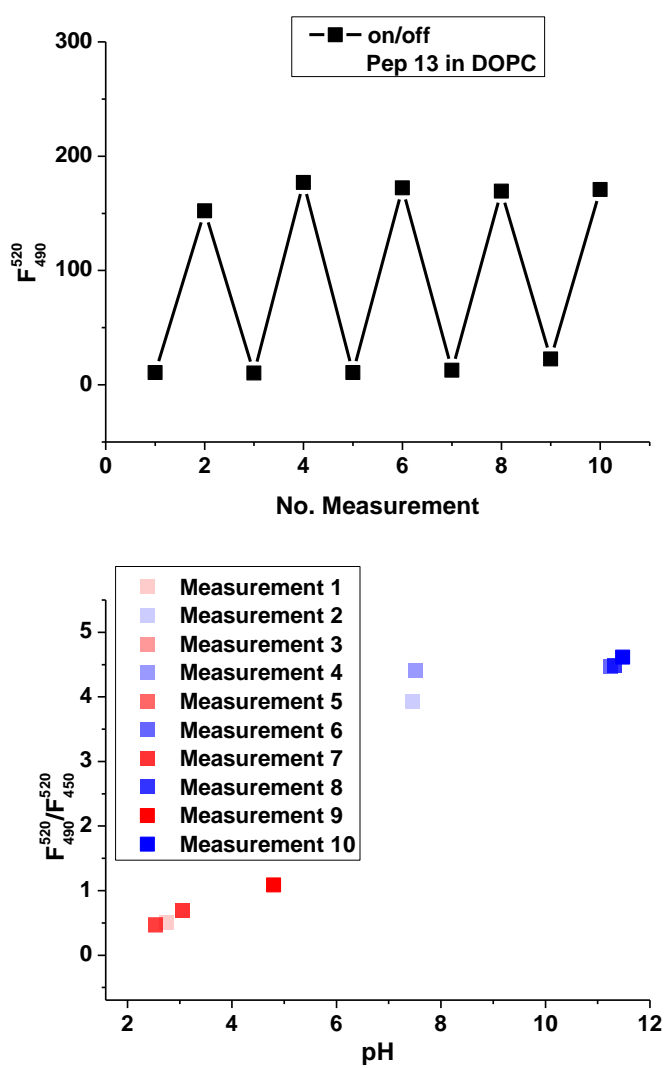
c) Pep 14 in DOPC
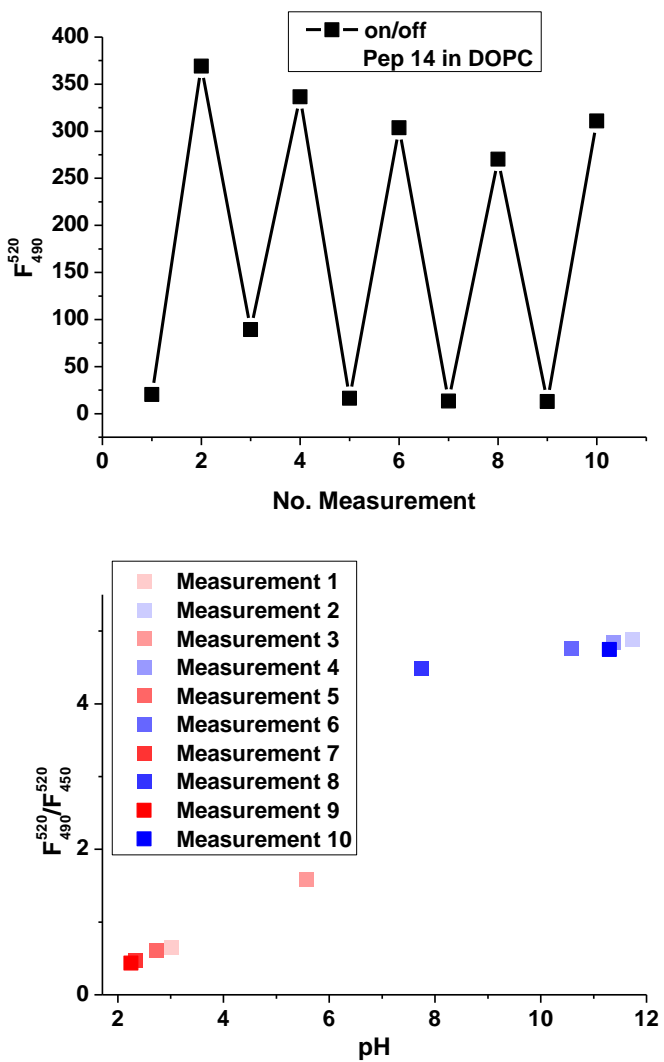

d) Pep 14 in DOPC/DOPG (50:50)
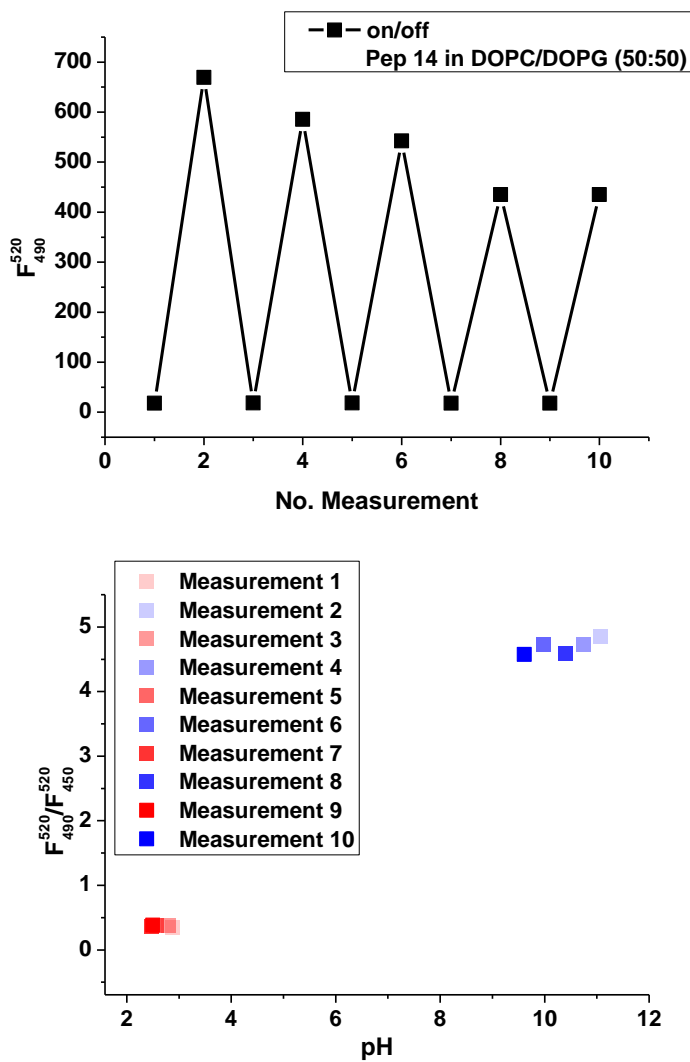

Figure 6.20 Fluorescence measurements of Pep 13 and Pep 14 in DOPC and DOPC/DOPG (50:50) vesicles rehydrated and extruded in PBS buffer with multiplied pH steps. 
$\AA$

$2 D_{c}$

$6 \mathrm{MH}$

A

AA

Ac

$\mathrm{Ac}_{2} \mathrm{O}$

$\mathrm{ACHC}$

ACHC

$\mathrm{ACN}$

Ala

ART-IR

Asn

ATP

ATR

BAPTA

BD

BIIC

Boc

BTIC

$\mathrm{Ca}^{2+}$

CCCP

$\mathrm{CD}$

D

DBU

DCM

DHPE

DIC

DIPEA

DLS

DMCM

DMF

DMNP-EDTA

DMPC

\section{Abbreviations}

Ångström

hydrophobic region

6-mercaptohexanol

electron acceptor group

amino acids

acetyl

acetic anhydride

trans-2-aminocyclohexanecarboxylic acid

trans-2-aminocyclohexanecarboxylic acid

acetonitrile

alanine

attenuated total reflection

asparagine

adenosine triphosphate

attenuated total reflection

1,2-bis( $o$-aminophenoxy)ethane- $N, N, N^{\prime}, N^{\prime}$-tetraacetic acid

Becton Dickinson

3-(benzimidazolyl)-iminocoumarin

tert-butoxycarbonyl

3-(benzothiazolyl)iminocoumarin

calcium ion

carbonyl cyanide $m$-chlorophenyl hydrazone

circular dichroism

electron donating group

1,8-diazabicyclo[5.4.0]undec-7-ene

dichlormethane

1,2-dihexadecanoyl-sn-glycero-3-phosphoethanolamine

$N, N^{\prime}$-diisopropylcarbodiimide

$\mathrm{N}, \mathrm{N}$-diisopropylethylamine

dynamic light scattering

(6,7-dimethoxycoumarin-4-yl)methyl

$N, N$-dimethylformamide

1-(4,5-dimethoxy-2-nitrophenyl)-1,2-diaminoethane- $N, N, N^{\prime}, N^{\prime}$ -

tetraacetic acid

1,2-dimyristoyl-sn-glycero-3-phosphocholine 


\begin{tabular}{|c|c|}
\hline DMSO & dimethyl sulfoxide \\
\hline DNP & dinitrophenylhydrazine \\
\hline DOPA & 1,2-dioleoyl-sn-glycero-3-phosphate \\
\hline DOPC & 1,2-dioleoyl-sn-glycero-3-phosphocholine \\
\hline DOPE & 1,2-dioleoyl-sn-glycero-3-phosphoethanolamine \\
\hline DOPG & 1,2-dioleoyl-sn-glycero-3-phosphoglycerol \\
\hline DOPS & 1,2-dioleoyl-sn-glycero-3-phospho-L-serine \\
\hline DOXYL & 4,4-dimethyl-3-oxazolidinyloxy \\
\hline DPC & dodecyl phosphocholine \\
\hline DPPC & 1,2-dipalmitoyl-sn-glycero-3-phosphocholine \\
\hline$D_{\mathrm{s}}$ & diffusion coefficient \\
\hline $\mathrm{EE}$ & ethyl acetate \\
\hline EGTA & ethylene glycol-bis(2-aminoethylether)- $N, N, N^{\prime}, N^{\prime}$-tetraacetic acid \\
\hline EI & electron ionisation \\
\hline eq & equivalents \\
\hline ESI & electrospray-ionisation \\
\hline ESI & electrospray ionization \\
\hline EtOAc & ethyl acetate \\
\hline FCS & fluorescence correlation spectroscopy \\
\hline Fmoc & fluorenylmethoxycarbonyl \\
\hline FRET & FÖRSTER resonance energy transfer \\
\hline FTIR & fourier-transform infrared spectroscopy \\
\hline Gln & glutamine \\
\hline $\mathrm{H}^{+}$ & proton \\
\hline HATU & $O$-(7-azabenzotriazol-1-yl)- $N, N, N^{\prime}, N^{\prime}$-tetramethyluronium \\
\hline & hexafluorophosphate \\
\hline HBTU & $O$-(1H-benzotriazol-1-yl)- $N, N, N^{\prime}, N^{\prime}$-tetramethyluronium \\
\hline & hexafluorophosphate \\
\hline HEPES & 4-(2-hydroxyethyl)-1-piperazineethanesulfonic acid \\
\hline HEPES & 4-(2-hydroxyethyl)-1-piperazineethanesulfonic acid \\
\hline HFIP & hexafluoro-2-propanol \\
\hline HOAt & 1-hydroxy-7-azabenzotriazole \\
\hline HOBt & 1-hydroxybenzotriazole \\
\hline HOMO & highest occupied molecular orbital \\
\hline HPLC & high performance liquid chromatography \\
\hline HR & high resolution \\
\hline
\end{tabular}




\begin{tabular}{|c|c|}
\hline Ile & isoleucine \\
\hline IR & infrared spectroscopy \\
\hline IRE & internal reflection element \\
\hline$J$ & coupling constant \\
\hline$K_{\mathrm{d}}$ & dissociation constant \\
\hline Leu & leucine \\
\hline LUMO & lowest unoccupied molecular orbital \\
\hline LUV & large unilamellar vesicle \\
\hline Lys & lysine \\
\hline $\mathrm{m} / \mathrm{z}$ & mass-to-charge ratio \\
\hline MBHA & 4-methylbenzhydrylamine \\
\hline MD & molecular-dynamics \\
\hline $\mathrm{MeOH}$ & methanol \\
\hline MIET & metal-induced energy transfer \\
\hline mif & membrane interaction factor \\
\hline МOBНА & $\begin{array}{l}\text { 2-(2'-morpholino-2'-oxoethoxy)- } N, N \text {-bis(hydroxycarbonylmethyl)- } \\
\text { aniline }\end{array}$ \\
\hline MS & mass spectrometry \\
\hline Mtt & 4-methyltrityl \\
\hline NBD & 7-nitrobenz-2-oxa-1,3-diazol-4-yl \\
\hline NMP & $N$-methyl-pyrrolidone \\
\hline NMR & nuclear magnetic resonance \\
\hline NP-EGTA & $o$-nitrophenyl EGTA \\
\hline Oxyma & ethyl cyanohydroxyiminoacetate \\
\hline $\mathrm{P} / \mathrm{L}$ & peptide-to-lipid \\
\hline PBS & phosphate buffered saline \\
\hline PCT & photoinduced charge transfer \\
\hline PE & phosphoethanolamine \\
\hline PEG & polyethylene glycol \\
\hline PET & photoinduced electron transfer \\
\hline $\mathrm{p} K_{\mathrm{a}}$ & acid dissociation constant \\
\hline ppm & parts per million \\
\hline PyAOP & $\begin{array}{l}\text { (7-azabenzotriazol-1-yloxy)tripyrrolidinophosphonium } \\
\text { hexafluorophosphate }\end{array}$ \\
\hline РyBOP & $\begin{array}{l}\text { benzotriazole-1-yl-oxy-tris-pyrrolodino-phosphonium } \\
\text { hexafluorophosphate }\end{array}$ \\
\hline
\end{tabular}




\begin{tabular}{|c|c|}
\hline $\mathrm{RP}$ & reversed phase \\
\hline rpm & rotations per minute \\
\hline RT & room temperature \\
\hline SAM & self assembled monolayer \\
\hline SEIRA & surface-enhanced infrared absorption \\
\hline Ser & serine \\
\hline SNARE & soluble N-ethylmaleimide-sensitive factor attachment protein receptor \\
\hline SNARF & seminaphtharhodafluor \\
\hline SOP & standard operating procedure \\
\hline SPPS & solid phase peptide synthesis \\
\hline SUV & small unilamellar vesicle \\
\hline SUV & small unilamellar vesicle \\
\hline$t$ & time \\
\hline T3P & propylphosphonic anhydride \\
\hline$t \mathrm{Bu}$ & tert-butyl protection group \\
\hline TFA & trifluoroacetic acid \\
\hline TFE & trifluoroethanol \\
\hline THF & tetrahydrofuran \\
\hline TIS & triisopropylsilan \\
\hline TLC & thin-layer chromatography \\
\hline TMS & trimethylsilyl \\
\hline$t_{\mathrm{R}}$ & retention time \\
\hline $\operatorname{Trp}$ & tryptophan \\
\hline UV & ultraviolet \\
\hline$v / v$ & volume/volume ratio \\
\hline Val & valine \\
\hline Vis & visual \\
\hline Vis & visual \\
\hline WK3SH & (2-(2-(2-ethoxy)ethoxy)ethanethiol \\
\hline$\chi$ & mole fraction \\
\hline$\delta$ & chemical shift (NMR) \\
\hline$\varepsilon$ & molar attenuation coefficient \\
\hline$\lambda$ & wavelength \\
\hline
\end{tabular}




\section{References}

[1] J. M. Berg, L. Stryer, J. L. Tymoczko, Stryer Biochemie, Springer Spektrum, Berlin Heidelberg, 2012.

[2] J. P. Overington, B. Al-Lazikani, A. L. Hopkins, Nat. Rev. Drug Discov. 2006, 5, 993-996.

[3] E. Wallin, G. von Heijne, Protein Sci. Publ. Protein Soc. 1998, 7, 1029-1038.

[4] A. Elofsson, G. von Heijne, Annu. Rev. Biochem. 2007, 76, 125-140.

[5] G. von Heijne, J. Intern. Med. 2007, 261, 543-557.

[6] M. Pelay-Gimeno, A. Glas, O. Koch, T. N. Grossmann, Angew. Chem. Int. Ed. 2015, 54, 8896-8927.

[7] A. Holt, J. A. Killian, Eur. Biophys. J. 2010, 39, 609-621.

[8] M. R. R. de Planque, J. A. Killian, Mol. Membr. Biol. 2003, 20, 271-284.

[9] E. Juaristi, V. A. Soloshonok, Enantioselective Synthesis of Beta-Amino Acids, John Wiley \& Sons, Hoboken, N.J, 2005.

[10] R. P. Cheng, S. H. Gellman, W. F. DeGrado, Chem. Rev. 2001, 101, 3219-3232.

[11] U. Rost, Organisation and Recognition of Artificial Transmembrane Peptides, Universität Göttingen, 2016.

[12] D. Petersen, Konformation Und Orientierung von Synthetisierten $\beta$-Peptiden in Membransystemen, Georg-August-Universität Göttingen, 2015.

[13] D. Zanbot, Dynamics, Intermolecular Interactions, and Organization of Transmembrane $\beta$-Peptides, Georg-August-Universität Göttingen, 2018.

[14] D. M. Pahlke, U. Diederichsen, J. Pept. Sci. 2016, 22, 636-641.

[15] J. Wegner, Synthesis of Rigid Spin Labels for the Investigation of Transmembrane Peptides by EPR Spectroscopy, Georg-August-Universität Göttingen, 2018.

[16] J. Ren, S. Lew, Z. Wang, E. London, Biochemistry 1997, 36, 10213-10220.

[17] J. Ren, S. Lew, J. Wang, E. London, Biochemistry 1999, 38, 5905-5912.

[18] J. T. Vivian, P. R. Callis, Biophys. J. 2001, 80, 2093-2109.

[19] S. Lew, J. Ren, E. London, Biochemistry 2000, 39, 9632-9640.

[20] L. A. Chung, J. D. Lear, W. F. DeGrado, Biochemistry 1992, 31, 6608-6616.

[21] J. R. Lakowicz, Topics in Fluorescence Spectroscopy, Kluwer Academic, New York, 2002.

[22] F. S. Abrams, E. London, Biochemistry 1993, 32, 10826-10831.

[23] E. J. Bolen, P. W. Holloway, Biochemistry 1990, 29, 9638-9643.

[24] M. Möller, A. Denicola, Biochem. Mol. Biol. Educ. 2002, 30, 175-178.

[25] A. Chattopadhyay, E. London, Biochemistry 1987, 26, 39-45.

[26] K. Ataka, S. T. Stripp, J. Heberle, Biochim. Biophys. Acta BBA - Biomembr. 2013, 1828, 2283-2293.

[27] E. Zaitseva, M. Saavedra, S. Banerjee, T. P. Sakmar, R. Vogel, Biophys. J. 2010, 99, 2327-2335.

[28] J. Kundu, F. Le, P. Nordlander, N. J. Halas, Chem. Phys. Lett. 2008, 452, 115-119.

[29] R. Kraayenhof, G. J. Sterk, H. W. Wong Fong Sang, Biochemistry 1993, 32, 1005710066.

[30] M. Schwamborn, Establishment of a Fluorescence Assay for Characterization of Protein-Mediated Vesicle Fusion and Acidification, 2017.

[31] T. Sanden, L. Salomonsson, P. Brzezinski, J. Widengren, Proc. Natl. Acad. Sci. 2010, 107, 4129-4134.

[32] L. Öjemyr, T. Sandén, J. Widengren, P. Brzezinski, Biochemistry 2009, 48, $2173-$ 2179.

[33] L. Xu, L. N. Öjemyr, J. Bergstrand, P. Brzezinski, J. Widengren, Biophys. J. 2016, 110, 1993-2003. 
[34] M. Brändén, T. Sandén, P. Brzezinski, J. Widengren, Proc. Natl. Acad. Sci. 2006, 103, 19766-19770.

[35] E. F. Etter, A. Minta, M. Poenie, F. S. Fay, Proc. Natl. Acad. Sci. 1996, 93, 53685373.

[36] L. A. Blatter, E. Niggli, Cell Calcium 1998, 23, 269-279.

[37] F. Liepouri, T. G. Deligeorgiev, Z. Veneti, C. Savakis, H. E. Katerinopoulos, Cell Calcium 2002, 31, 221-227.

[38] U. Rost, Y. Xu, T. Salditt, U. Diederichsen, ChemPhysChem 2016, 17, 2525-2534.

[39] A. B. Hughes, Ed. , Amino Acids, Peptides and Proteins in Organic Chemistry: Origins and Synthesis of Amino Acids, Wiley-VCH Verlag GmbH \& Co. KGaA, Weinheim, Germany, 2009.

[40] D. Seebach, A. K. Beck, D. J. Bierbaum, Chem. Biodivers. 2004, 1, 1111-1239.

[41] H. Tomita, Y. Yokooji, T. Ishibashi, T. Imanaka, H. Atomi, J. Bacteriol. 2014, 196, $1222-1230$.

[42] D. Seebach, J. Gardiner, Acc. Chem. Res. 2008, 41, 1366-1375.

[43] W. F. DeGrado, J. P. Schneider, Y. Hamuro, J. Pept. Res. 1999, 54, 206-217.

[44] F. von Nussbaum, P. Spiteller, Highlights Bioorganic Chem. 2005, DOI 10.1002/3527603727.ch1e.

[45] J. B. Stock, A. J. Ninfa, A. M. Stock, Microbiol. Rev. 1989, 53, 450-490.

[46] Z.-Z. Chen, B. Tan, Y.-M. Li, Y.-F. Zhao, Int. J. Quantum Chem. 2001, 83, 41-51.

[47] Z.-Z. Chen, B. Tan, Y.-M. Li, Y.-F. Zhao, Y.-F. Tong, J.-F. Wang, J. Org. Chem. 2003, 68, 4052-4058.

[48] M. del P. García-Santos, S. González-Mancebo, J. Hernández-Benito, E. Calle, J. Casado, J. Am. Chem. Soc. 2002, 124, 2177-2182.

[49] S. S. Mirvish, Cancer Lett. 1995, 93, 17-48.

[50] D. H. Appella, L. A. Christianson, D. A. Klein, D. R. Powell, X. Huang, J. J. Barchi, S. H. Gellman, Nature 1997, 387, 381-384.

[51] S. H. Gellman, Acc. Chem. Res. 1998, 31, 173-180.

[52] D. H. Appella, L. A. Christianson, I. L. Karle, D. R. Powell, S. H. Gellman, J. Am. Chem. Soc. 1996, 118, 13071-13072.

[53] T. L. Raguse, E. A. Porter, B. Weisblum, S. H. Gellman, J. Am. Chem. Soc. 2002, 124, 12774-12785.

[54] G. Lelais, D. Seebach, B. Jaun, R. I. Mathad, O. Flögel, F. Rossi, M. Campo, A. Wortmann, Helv. Chim. Acta 2006, 89, 361-403.

[55] A. Hayen, M. A. Schmitt, F. N. Ngassa, K. A. Thomasson, S. H. Gellman, Angew. Chem. 2004, 116, 511-516.

[56] G. V. M. Sharma, P. Nagendar, P. Jayaprakash, P. Radha Krishna, K. V. S. Ramakrishna, A. C. Kunwar, Angew. Chem. 2005, 117, 6028-6032.

[57] Methods Enzymol. 2013, 523, 407-429.

[58] L. M. Johnson, S. H. Gellman, Methods Enzymol. 2013, 523, 407-429.

[59] M. A. Schmitt, B. Weisblum, S. H. Gellman, J. Am. Chem. Soc. 2004, 126, 68486849.

[60] D. F. Hook, P. Bindschädler, Y. R. Mahajan, R. Šebesta, P. Kast, D. Seebach, Chem. Biodivers. 2005, 2, 591-632.

[61] Y.-D. Wu, W. Han, D.-P. Wang, Y. Gao, Y.-L. Zhao, Acc. Chem. Res. 2008, 41, 1418-1427.

[62] D. Seebach, S. Abele, K. Gademann, G. Guichard, T. Hintermann, B. Jaun, J. L. Matthews, J. V. Schreiber, L. Oberer, U. Hommel, et al., Helv. Chim. Acta 1998, 81, 932-982.

[63] T. Beke, C. Somlai, A. Perczel, J. Comput. Chem. 2006, 27, 20-38. 
[64] X. Wang, J. F. Espinosa, S. H. Gellman, J. Am. Chem. Soc. 2000, 122, 4821-4822.

[65] T. L. Raguse, J. R. Lai, P. R. LePlae, S. H. Gellman, Org. Lett. 2001, 3, 3963-3966.

[66] F. Gessier, C. Noti, M. Rueping, D. Seebach, Helv. Chim. Acta 2003, 86, 1862-1870.

[67] D. Seebach, M. Overhand, F. N. Kühnle, B. Martinoni, L. Oberer, U. Hommel, H. Widmer, Helv. Chim. Acta 1996, 79, 913-941.

[68] J. Gardiner, R. I. Mathad, B. Jaun, J. V. Schreiber, O. Flögel, D. Seebach, Helv. Chim. Acta 2009, 92, 2698-2721.

[69] D. A. Niggli, M.-O. Ebert, Z. Lin, D. Seebach, W. F. van Gunsteren, Chem. - Eur. J. 2012, 18, 586-593.

[70] D. Seebach, J. V. Schreiber, S. Abele, X. Daura, W. F. van Gunsteren, Helv. Chim. Acta 2000, 83, 34-57.

[71] M. J. Koyack, R. P. Cheng, Design and Synthesis of $\beta$-Peptides With Biological Activity, Humana Press, New Jersey, 2006.

[72] R. Srivastava, A. Kumar Ray, U. Diederichsen, Eur. J. Org. Chem. 2009, 2009, 4793-4800.

[73] N. Diezemann, L. M. M. Weigang, P. Chakraborty, H. Frauendorf, T. Letzel, U. Diederichsen, J. Mass Spectrom. 2009, 44, 794-802.

[74] J. A. Kritzer, J. Tirado-Rives, S. A. Hart, J. D. Lear, W. L. Jorgensen, A. Schepartz, J. Am. Chem. Soc. 2005, 127, 167-178.

[75] P. R. LePlae, J. D. Fisk, E. A. Porter, B. Weisblum, S. H. Gellman, J. Am. Chem. Soc. 2002, 124, 6820-6821.

[76] W. C. Pomerantz, T. L. R. Grygiel, J. R. Lai, S. H. Gellman, Org. Lett. 2008, 10, 1799-1802.

[77] M. Lee, T. L. Raguse, M. Schinnerl, W. C. Pomerantz, X. Wang, P. Wipf, S. H. Gellman, Org. Lett. 2007, 9, 1801-1804.

[78] J. Applequist, K. A. Bode, D. H. Appella, L. A. Christianson, S. H. Gellman, J. Am. Chem. Soc. 1998, 120, 4891-4892.

[79] S. H. Choi, I. A. Guzei, L. C. Spencer, S. H. Gellman, J. Am. Chem. Soc. 2010, 132, 13879-13885.

[80] K. A. Bode, J. Applequist, Macromolecules 1997, 30, 2144-2150.

[81] K. Kaur, T. Sprules, W. Soliman, R. Beleid, S. Ahmed, Biochim. Biophys. Acta BBA Proteins Proteomics 2008, 1784, 658-665.

[82] D. S. Daniels, E. J. Petersson, J. X. Qiu, A. Schepartz, J. Am. Chem. Soc. 2007, 129, $1532-1533$

[83] T. L. Raguse, J. R. Lai, S. H. Gellman, Helv. Chim. Acta 2002, 85, 4154-4164.

[84] A. Glättli, D. Seebach, W. F. van Gunsteren, Helv. Chim. Acta 2004, 87, 2487-2506.

[85] S. Abele, G. Guichard, D. Seebach, Helv. Chim. Acta 1998, 81, 2141-2156.

[86] R. Jahn, R. H. Scheller, Nat. Rev. Mol. Cell Biol. 2006, 7, 631-643.

[87] W. A. Catterall, Annu. Rev. Biochem. 1995, 64, 493-531.

[88] L. Wiefel, A. Steinbuchel, Appl. Environ. Microbiol. 2014, 80, 1091-1096.

[89] M. R. de Planque, J. A. Kruijtzer, R. M. Liskamp, D. Marsh, D. V. Greathouse, R. E. Koeppe, B. de Kruijff, J. A. Killian, J. Biol. Chem. 1999, 274, 20839-20846.

[90] B. S. Patil, G.-R. Vasanthakumar, V. V. S. Babu, Lett. Pept. Sci. 2002, 9, 231-233.

[91] Kantharaju, B. S. Patil, V. V. S. Babu, IJC-B Vol44B12 Dec. 20052005.

[92] L. A. Carpino, G. Y. Han, J. Org. Chem. 1972, 37, 3404-3409.

[93] B. Marglin, R. B. Merrifield, J. Am. Chem. Soc. 1966, 88, 5051-5052.

[94] U. Rost, C. Steinem, U. Diederichsen, Chem. Sci. 2016, 7, 5900-5907.

[95] M. S. Bernatowicz, S. B. Daniels, H. Köster, Tetrahedron Lett. 1989, 30, 4645-4648.

[96] Y.-P. Zhang, R. N. A. H. Lewis, G. D. Henry, B. D. Sykes, R. S. Hodges, R. N. McElhaney, Biochemistry 1995, 34, 2348-2361. 
[97] A. Holt, R. B. M. Koehorst, T. Rutters-Meijneke, M. H. Gelb, D. T. S. Rijkers, M. A. Hemminga, J. A. Killian, Biophys. J. 2009, 97, 2258-2266.

[98] J. M. Collins, K. A. Porter, S. K. Singh, G. S. Vanier, Org. Lett. 2014, 16, 940-943.

[99] S. Abu-Baker, P. Garber, B. Hina, T. Reed, G. Shahrokh, M. Al-Saghir, G. Lorigan, Open J. Synth. Theory Appl. 2014, 03, 1-4.

[100] J. M. Collins, S. K. Singh, G. S. Vanier, Chem Today 2012, 30.

[101] J. K. Murray, B. Farooqi, J. D. Sadowsky, M. Scalf, W. A. Freund, L. M. Smith, J. Chen, S. H. Gellman, J. Am. Chem. Soc. 2005, 127, 13271-13280.

[102] J. K. Murray, S. H. Gellman, Org. Lett. 2005, 7, 1517-1520.

[103] H. Franzén, L. Grehn, U. Ragnarsson, J. Chem. Soc. Chem. Commun. 1984, O, 1699-1700.

[104] J. M. Collins, S. K. Singh, Coupling Method for Peptide Synthesis at Elevated Temperatures, 2016, US2016176918 (A1).

[105] J. M. Collins, S. K. Singh, Use of Excess Carbodiimide for Peptide Synthesis at Elevated Temperatures, 2018, US2018066013 (A1).

[106] S. K. Singh, J. M. Collins, "Fmoc Solid Phase Microwave Synthesis of Long Peptides," can be found under

http://www.cem.de/documents/produkte/peptid/525_Poster_LibBlue_2014EPS_Fmoc SPPSLongPeptides.pdf, n.d.

[107] G. Lelais, Synthesis of New $\beta$-Amino Acids and Novel $\beta$-Peptides, and Structural Investigations, Eidgenössische Technische Hochschule Zürich, 2004.

[108] A. Caporale, N. Doti, A. Sandomenico, M. Ruvo, J. Pept. Sci. 2017, 23, 272-281.

[109] T. Hara, Y. Huang, A. Ito, T. Kawakami, H. Hojo, M. Murata, J. Pept. Sci. 2014, $21,61-70$.

[110] J. Rizo, F. Albericio, E. Giralt, E. Pedroso, Tetrahedron Lett. 1992, 33, 397-400.

[111] G. Richter, Praktische Biochemie: Grundlagen und Techniken ; 19 Tabellen, Georg Thieme Verlag, 2003.

[112] E. Pazos, O. Vázquez, J. L. Mascareñas, M. E. Vázquez, Chem. Soc. Rev. 2009, 38, 3348-3359.

[113] B. Valeur, M. N. Berberan-Santos, Molecular Fluorescence: Principles and Applications, John Wiley \& Sons, 2013.

[114] N. Naarmann, Charakterisierung Und Kontrolle Selbstorganisierter Peptidehelixbündel in Phospholipidmembranen, 2005.

[115] H.-J. Galla, Spektroskopische Methoden in der Biochemie: 13 Tab, Thieme, Stuttgart, 1988.

[116] C. S. McKay, M. G. Finn, Chem. Biol. 2014, 21, 1075-1101.

[117] X. Chen, Y.-W. Wu, Org. Biomol. Chem. 2016, 14, 5417-5439.

[118] C. P. Toseland, J. Chem. Biol. 2013, 6, 85-95.

[119] L. Wang, J. Xie, P. G. Schultz, Annu. Rev. Biophys. Biomol. Struct. 2006, 35, 225249.

[120] L. Yin, W. Wang, S. Wang, F. Zhang, S. Zhang, N. Tao, Biosens. Bioelectron. 2015, 66, 412-416.

[121] A. Groß, C. Hashimoto, H. Sticht, J. Eichler, Front. Bioeng. Biotechnol. 2016, 3, DOI 10.3389/fbioe.2015.00211.

[122] R. Behrendt, P. White, J. Offer, J. Pept. Sci. 2016, 22, 4-27.

[123] J. Yin, Y. Hu, J. Yoon, Chem Soc Rev 2015, 44, 4619-4644.

[124] P. Jiang, Z. Guo, Coord. Chem. Rev. 2004, 248, 205-229.

[125] L. Basabe-Desmonts, D. N. Reinhoudt, M. Crego-Calama, Chem. Soc. Rev. 2007, 36, 993-1017.

[126] Y. Yan, G. Marriott, Curr. Opin. Chem. Biol. 2003, 7, 635-640. 
[127] A. J. de Jesus, T. W. Allen, Biochim. Biophys. Acta BBA - Biomembr. 2013, 1828, 864-876.

[128] V. V. Vostrikov, R. E. Koeppe, Biochemistry 2011, 50, 7522-7535.

[129] Y. Chen, M. D. Barkley, Biochemistry 1998, 37, 9976-9982.

[130] F. Scarpelli, M. Drescher, T. Rutters-Meijneke, A. Holt, D. T. S. Rijkers, J. A. Killian, M. Huber, J. Phys. Chem. B 2009, 113, 12257-12264.

[131] J. A. Killian, I. Salemink, M. R. R. de Planque, G. Lindblom, R. E. Koeppe, D. V. Greathouse, Biochemistry 1996, 35, 1037-1045.

[132] J. M. Rankenberg, V. V. Vostrikov, D. V. Greathouse, C. V. Grant, S. J. Opella, R. E. Koeppe, Biochemistry 2012, 51, 10066-10074.

[133] Biochim. Biophys. Acta BBA - Biomembr. 2006, 1758, 1184-1202.

[134] D. A. Dougherty, Science 1996, 271, 163-168.

[135] W. C. Wimley, S. H. White, Nat. Struct. Biol. 1996, 3, 842-848.

[136] W.-M. Yau, W. C. Wimley, K. Gawrisch, S. H. White, Biochemistry 1998, 37, 14713-14718.

[137] B. A. Lewis, D. M. Engelman, J. Mol. Biol. 1983, 166, 211-217.

[138] N. Kučerka, Y. Liu, N. Chu, H. I. Petrache, S. Tristram-Nagle, J. F. Nagle, Biophys. J. 2005, 88, 2626-2637.

[139] J. F. Nagle, S. Tristram-Nagle, Biochim. Biophys. Acta BBA - Rev. Biomembr. 2000, 1469, 159-195.

[140] N. Kučerka, M.-P. Nieh, J. Katsaras, Biochim. Biophys. Acta BBA - Biomembr. 2011, 1808, 2761-2771.

[141] M. A. Schuler, I. G. Denisov, S. G. Sligar, Methods Mol. Biol. Clifton NJ 2013, 974, 415-433.

[142] L. E. Cybulski, J. Ballering, A. Moussatova, M. E. Inda, D. B. Vazquez, T. A. Wassenaar, D. de Mendoza, D. P. Tieleman, J. A. Killian, Proc. Natl. Acad. Sci. 2015, 112, 6353-6358.

[143] F. N. Barrera, D. Weerakkody, M. Anderson, O. A. Andreev, Y. K. Reshetnyak, D. M. Engelman, J. Mol. Biol. 2011, 413, 359-371.

[144] D. Seebach, P. E. Ciceri, M. Overhand, B. Jaun, D. Rigo, L. Oberer, U. Hommel, R. Amstutz, H. Widmer, Helv. Chim. Acta 1996, 79, 2043-2066.

[145] S. Kabatas, Synthese Artifizieller Peptide zur Erzeugung von Supramolekularen Architekturen und zur Markierung von Biomolekülen, Cuvillier Verlag, 2016.

[146] C. Fernandes, S. Faure, E. Pereira, V. Théry, V. Declerck, R. Guillot, D. J. Aitken, Org. Lett. 2010, 12, 3606-3609.

[147] A. Altmayer-Henzien, V. Declerck, J. Farjon, D. Merlet, R. Guillot, D. J. Aitken, Angew. Chem. Int. Ed. 2015, 54, 10807-10810.

[148] M. Rueping, Y. R. Mahajan, B. Jaun, D. Seebach, Chem. - Eur. J. 2004, 10, 16071615.

[149] J.-S. Park, H.-S. Lee, J. R. Lai, B. M. Kim, S. H. Gellman, J. Am. Chem. Soc. 2003, $125,8539-8545$.

[150] T. L. Raguse, J. R. Lai, S. H. Gellman, J. Am. Chem. Soc. 2003, 125, 5592-5593.

[151] N. Kučerka, S. Tristram-Nagle, J. F. Nagle, J. Membr. Biol. 2006, 208, 193-202.

[152] N. Castillo, L. Monticelli, J. Barnoud, D. P. Tieleman, Chem. Phys. Lipids 2013, $169,95-105$.

[153] N. Abdul-Manan, J. F. Hinton, Biochemistry 1994, 33, 6773-6783.

[154] F. D. Sonnichsen, J. E. Van Eyk, R. S. Hodges, B. D. Sykes, Biochemistry 1992, 31, 8790-8798.

[155] B. A. Wallace, Protein Sci. 2003, 12, 875-884. 
[156] Y. Hamuro, J. P. Schneider, W. F. DeGrado, J. Am. Chem. Soc. 1999, 121, 1220012201.

[157] J. R. Lakowicz, Principles of Fluorescence Spectroscopy, Springer, New York, 2010.

[158] N. Höfer, D. Aragão, M. Caffrey, Biophys. J. 2010, 99, L23-L25.

[159] M. Caffrey, V. Cherezov, Nat. Protoc. 2009, 4, 706-731.

[160] K.-J. Shon, Y. Kim, L. A. Colnago, S. J. Opella, Science 1991.

[161] S.-T. Bogen, G. de Korte-Kool, G. Lindblom, L. B.-Å. Johansson, J. Phys. Chem. B 1999, 103, 8344-8352.

[162] M. Iqbal, P. Balaram, Biopolymers 1982, 21, 1427-1433.

[163] R. Winter, F. Noll, C. Czeslik, Methoden der biophysikalischen Chemie, Vieweg + Teubner, Wiesbaden, 2011.

[164] M. Kondo, M. Mehiri, S. L. Regen, J. Am. Chem. Soc. 2008, 130, 13771.

[165] J. Galbán, E. Mateos, V. Cebolla, A. Domínguez, A. Delgado-Camón, S. de

Marcos, I. Sanz-Vicente, V. Sanz, The Analyst 2009, 134, 2286.

[166] F.-X. Ding, H. Xie, B. Arshava, J. M. Becker, F. Naider, Biochemistry 2001, 40, 8945-8954.

[167] Y. Shai, Biochim. Biophys. Acta BBA - Biomembr. 2013, 1828, 2306-2313.

[168] C. Vigano, L. Manciu, F. Buyse, E. Goormaghtigh, J. M. Ruysschaert, Biopolymers 2000, 55, 373-380.

[169] M. Osawa, Bull. Chem. Soc. Jpn. 1997, 70, 2861-2880.

[170] E. Forbrig, J. K. Staffa, J. Salewski, M. A. Mroginski, P. Hildebrandt, J. Kozuch, Langmuir 2018, 34, 2373-2385.

[171] M. Osawa, Handb. Vib. Spectrosc. 2006, 15.

[172] G. Schkolnik, J. Salewski, D. Millo, I. Zebger, S. Franzen, P. Hildebrandt, Int. J. Mol. Sci. 2012, 13, 7466-7482.

[173] J. K. Staffa, L. Lorenz, M. Stolarski, D. H. Murgida, I. Zebger, T. Utesch, J.

Kozuch, P. Hildebrandt, J. Phys. Chem. C 2017, 121, 22274-22285.

[174] S. D. Fried, S. G. Boxer, Acc. Chem. Res. 2015, 48, 998-1006.

[175] N. M. Levinson, S. D. Fried, S. G. Boxer, J. Phys. Chem. B 2012, 116, 10470 10476.

[176] S. D. Dalosto, J. M. Vanderkooi, K. A. Sharp, J. Phys. Chem. B 2004, 108, 64506457.

[177] V. Oklejas, C. Sjostrom, J. M. Harris, J. Am. Chem. Soc. 2002, 124, 2408-2409.

[178] V. Oklejas, J. M. Harris, Langmuir 2003, 19, 5794-5801.

[179] Z. Getahun, C.-Y. Huang, T. Wang, B. De León, W. F. DeGrado, F. Gai, J. Am. Chem. Soc. 2003, 125, 405-411.

[180] M. Maj, C. Ahn, D. Kossowska, K. Park, K. Kwak, H. Han, M. Cho, Phys. Chem. Chem. Phys. 2015, 17, 11770-11778.

[181] S. Ortgies, C. Depken, A. Breder, Org. Lett. 2016, 18, 2856-2859.

[182] A. L. L. García, Synlett 2007, 2007, 1328-1329.

[183] C. Millbanks, M. Bonnekessel, in Encycl. Reag. Org. Synth., John Wiley \& Sons, Ltd, 2001.

[184] M. Hesse, H. Meier, B. Zeeh, Spektroskopische Methoden in der organischen Chemie: 102 Tabellen, Thieme, Stuttgart, 2005.

[185] J. A. Kozuch, Structure-Function Relationships of Membrane Proteins Spectroelectrochemical Investigation of Artificial Membranes, 2013.

[186] N. Agmon, H. J. Bakker, R. K. Campen, R. H. Henchman, P. Pohl, S. Roke, M.

Thämer, A. Hassanali, Chem. Rev. 2016, 116, 7642-7672.

[187] J. Seelig, Cell Biol. Int. Rep. 1990, 14, 353-360. 
[188] A. Melcrová, S. Pokorna, S. Pullanchery, M. Kohagen, P. Jurkiewicz, M. Hof, P. Jungwirth, P. S. Cremer, L. Cwiklik, Sci. Rep. 2016, 6, 1-12.

[189] M. Luckey, Membrane Structural Biology: With Biochemical and Biophysical Foundations, Cambridge University Press, 2014.

[190] M. Anderson, A. Moshnikova, D. M. Engelman, Y. K. Reshetnyak, O. A. Andreev, Proc. Natl. Acad. Sci. 2016, 201608247.

[191] A. A. Pezzulo, X. X. Tang, M. J. Hoegger, M. H. A. Alaiwa, S. Ramachandran, T. O. Moninger, P. H. Karp, C. L. Wohlford-Lenane, H. P. Haagsman, M. van Eijk, et al., Nature 2012, 487, 109-113.

[192] W. Paschen, B. Djuricic, G. Mies, R. Schmidt-Kastner, F. Linn, J. Neurochem. 1987, 48, 154-159.

[193] R. R. Kedika, R. F. Souza, S. J. Spechler, Dig. Dis. Sci. 2009, 54, 2312-2317.

[194] P. D. Boyer, Annu. Rev. Biochem. 1997, 66, 717-749.

[195] E. Weichselbaum, M. Österbauer, D. G. Knyazev, O. V. Batishchev, S. A. Akimov, T. H. Nguyen, C. Zhang, G. Knör, N. Agmon, P. Carloni, et al., Sci. Rep. 2017, 7, 4553.

[196] S. Serowy, S. M. Saparov, Y. N. Antonenko, W. Kozlovsky, V. Hagen, P. Pohl, Biophys. J. 2003, 84, 1031-1037.

[197] V. Y. Evtodienko, Y. N. Antonenko, L. S. Yaguzhinsky, FEBS Lett. 1998, 425, 222-224.

[198] Y. N. Antonenko, G. A. Denisov, P. Pohl, Biophys. J. 1993, 64, 1701-1710.

[199] Y. N. Antonenko, A. A. Bulychev, Biochim. Biophys. Acta BBA - Biomembr. 1991, 1070, 279-282.

[200] V. Tvarozek, A. Ottova-Leitmannova, I. Novotny, V. Rehacek, F. Mika, H. T. Tien, PROC. 21 st International conference on microelectronics 1997, 2, 543-546.

[201] V. Borisenko, Z. Zhang, G. A. Woolley, Biochim. Biophys. Acta BBA-Biomembr. 2002, 1558, 26-33.

[202] D. E. Orosz, K. D. Garlid, Anal. Biochem. 1993, 210, 7-15.

[203] M. Schwamborn, J. Schumacher, J. Sibold, N. K. Teiwes, C. Steinem, The Analyst 2017, 142, 2670-2677.

[204] A. Springer, V. Hagen, D. A. Cherepanov, Y. N. Antonenko, P. Pohl, Proc. Natl. Acad. Sci. 2011, 108, 14461-14466.

[205] T. Hianik, V. I. Passechnik, Bilayer Lipid Membranes. Structure and Mechanical Properties, Springer Science \& Business Media, 1995.

[206] R. J. P. Williams, Annu. Rev. Biophys. Biophys. Chem. 1988, 17, 71-97.

[207] E. S. Medvedev, A. A. Stuchebrukhov, FEBS Lett. 2013, 587, 345-349.

[208] R. B. Gennis, Biophys. J. 2016, 110, 1909-1911.

[209] I. G. Denisov, S. G. Sligar, Chem. Rev. 2017, 117, 4669-4713.

[210] J. Han, K. Burgess, Chem. Rev. 2010, 110, 2709-2728.

[211] L. D. Hughes, R. J. Rawle, S. G. Boxer, PLoS ONE 2014, 9, e87649.

[212] F. Pincet, V. Adrien, R. Yang, J. Delacotte, J. E. Rothman, W. Urbach, D. Tareste, PLOS ONE 2016, 11, e0158457.

[213] K. Shi, Y. Long, C. Xu, Y. Wang, Y. Qiu, Q. Yu, Y. Liu, Q. Zhang, H. Gao, Z. Zhang, et al., ACS Appl. Mater. Interfaces 2015, 7, 21442-21454.

[214] S. Thutupalli, J.-B. Fleury, A. Steinberger, S. Herminghaus, R. Seemann, Chem. Commun. 2013, 49, 1443-1445.

[215] M. Adamczyk, J. R. Fishpaugh, K. J. Heuser, Bioconjug. Chem. 1997, 8, 253-255.

[216] M. Aschi, A. A. D’Archivio, A. Fontana, A. Formiglio, J. Org. Chem. 2008, 73, 3411-3417.

[217] M. M. Martin, L. Lindqvist, J. Lumin. 1975, 10, 381-390. 
[218] R. Sjöback, J. Nygren, M. Kubista, Spectrochim. Acta. A. Mol. Biomol. Spectrosc. 1995, 51, L7-L21.

[219] S. Massou, R. Albigot, M. Prats, Biochem. Educ. 2000, 28, 171-173.

[220] A. L. F. Baptista, P. J. G. Coutinho, M. E. C. D. Real Oliveira, J. I. N. Rocha Gomes, J. Liposome Res. 2003, 13, 123-130.

[221] S. Bidmanova, A. Hlavacek, J. Damborsky, Z. Prokop, Sens. Actuators B Chem. 2012, 161, 93-99.

[222] H. Siegumfeldt, K. B. Rechinger, M. Jakobsen, Microbiology 1999, 145, 17031709.

[223] I. Johnson, Ed. , The Molecular Probes Handbook: A Guide to Fluorescent Probes and Labeling Technologies, Life Technologies, Carlsbad, Calif., 2010.

[224] S. Mordon, J. M. Devoisselle, V. Maunoury, Photochem. Photobiol. 1994, 60, 274 279.

[225] R. F. Chen, J. R. Knutson, Anal. Biochem. 1988, 172, 61-77.

[226] R. M. Watt, E. W. Voss, J. Biol. Chem. 1979, 254, 1684-1690.

[227] Y. Kim, S. O. Ho, N. R. Gassman, Y. Korlann, E. V. Landorf, F. R. Collart, S. Weiss, Bioconjug. Chem. 2008, 19, 786-791.

[228] K. Halbmair, J. Wegner, U. Diederichsen, M. Bennati, Biophys. J. 2016, 111, $2345-2348$.

[229] R. Fischer, O. Mader, G. Jung, R. Brock, Bioconjug. Chem. 2003, 14, 653-660.

[230] P. J. Stahl, J. C. Cruz, Y. Li, S. Michael Yu, K. Hristova, Anal. Biochem. 2012, 424, 137-139.

[231] C. R. Wasmuth, C. Edwards, R. Hutcherson, J. Phys. Chem. 1964, 68, 423-425.

[232] V. B. Bajić, T. W. Tan, Information Processing and Living Systems, 2005.

[233] D. A. R. Paradkar, Biopharmaceutics \& Pharmacokinetics, Pragati Books Pvt. Ltd., 2008.

[234] I. Szundi, W. Stoeckenius, Biophys. J. 1989, 56, 369-383.

[235] C.-M. M. S. Geilfus, K. H. Muehling, Front. Plant Sci. 2011, 2, DOI 10.3389/fpls.2011.00013.

[236] R. Kraayenhof, G. J. Sterk, H. W. Wong Fong Sang, K. Krab, R. M. Epand, Biochim. Biophys. Acta BBA - Biomembr. 1996, 1282, 293-302.

[237] N. Karedla, A. I. Chizhik, I. Gregor, A. M. Chizhik, O. Schulz, J. Enderlein, ChemPhysChem 2014, 15, 705-711.

[238] A. I. Chizhik, J. Rother, I. Gregor, A. Janshoff, J. Enderlein, Nat. Photonics 2014, 8, 124-127.

[239] N. Karedla, A. M. Chizhik, S. C. Stein, D. Ruhlandt, I. Gregor, A. I. Chizhik, J. Enderlein, J. Chem. Phys. 2018, 148, 204201.

[240] I. M. Pazos, I. A. Ahmed, M. I. L. Berríos, F. Gai, Anal. Biochem. 2015, 483, 2126.

[241] A. K. Campbell, Intracellular Calcium, John Wiley \& Sons, 2014.

[242] K. W. Raymond, General Organic and Biological Chemistry, John Wiley \& Sons, 2009.

[243] A. Berk, C. A. Kaiser, H. Lodish, A. Amon, H. Ploegh, A. Bretscher, M. Krieger,

K. C. Martin, Molecular Cell Biology, WH Freeman, New York, 2016.

[244] S. A. Stricker, V. E. Centonze, S. W. Paddock, G. Schatten, Dev. Biol. 1992, 149, 370-380.

[245] M. Whitaker, Physiol. Rev. 2006, 86, 25-88.

[246] T. C. Südhof, Cold Spring Harb. Perspect. Biol. 2012, 4, a011353.

[247] Z. P. Pang, T. C. Südhof, Curr. Opin. Cell Biol. 2010, 22, 496-505.

[248] G. J. Augustine, Curr. Opin. Neurobiol. 2001, 11, 320-326. 
[249] M. J. Berridge, Nature 1993, 361, 315-325.

[250] M. J. Berridge, M. D. Bootman, P. Lipp, Nature 1998, 395, 645-648.

[251] K. P. Subedi, O. Paudel, J. S. K. Sham, Am. J. Physiol.-Cell Physiol. 2014, DOI 10.1152/ajpcell.00341.2013.

[252] N. Xu, M. Francis, D. L. Cioffi, T. Stevens, Am. J. Physiol.-Cell Physiol. 2014, DOI 10.1152/ajpcell.00046.2014.

[253] A. Lajtha, Chemical and Cellular Architecture, Springer Science \& Business Media, 2013.

[254] T. C. Sudhof, J. Rizo, Cold Spring Harb. Perspect. Biol. 2011, 3, a005637.

[255] V. Kiessling, S.-T. Yang, L. K. Tamm, Curr. Top. Membr. 2015, 75, 1-23.

[256] A. Yamaji-Hasegawa, M. Tsujimoto, Biol. Pharm. Bull. 2006, 29, 1547-1553.

[257] J. T. Littleton, J. Bai, B. Vyas, R. Desai, A. E. Baltus, M. B. Garment, S. D.

Carlson, B. Ganetzky, E. R. Chapman, J. Neurosci. 2001, 21, 1421-1433.

[258] B. Jena, Protein Pept. Lett. 2009, 16, 712-717.

[259] P. Jurkiewicz, L. Cwiklik, A. Vojtíšková, P. Jungwirth, M. Hof, Biochim. Biophys. Acta BBA - Biomembr. 2012, 1818, 609-616.

[260] H. I. Ingolfsson, P. Tieleman, S. Marrink, Biophys. J. 2015, 108, 14554-14559.

[261] M. N. Jones, Adv. Colloid Interface Sci. 1995, 54, 93-128.

[262] J. Melcr, D. Bonhenry, Š. Timr, P. Jungwirth, J. Chem. Theory Comput. 2016, 12, $2418-2425$.

[263] M. Javanainen, A. Melcrová, A. Magarkar, P. Jurkiewicz, M. Hof, P. Jungwirth, H. Martinez-Seara, Chem. Commun. 2017, 53, 5380-5383.

[264] J. W. Putney, Ed. , Calcium Signaling, CRC/Taylor \& Francis, Boca Raton, 2006.

[265] M. Oheim, M. van 't Hoff, A. Feltz, A. Zamaleeva, J.-M. Mallet, M. Collot,

Biochim. Biophys. Acta BBA - Mol. Cell Res. 2014, 1843, 2284-2306.

[266] R. Y. Tsien, Biochemistry 1980, 19, 2396-2404.

[267] J. T. Gerig, P. Singh, L. A. Levy, R. E. London, J. Inorg. Biochem. 1987, 31, 113121.

[268] H. J. Kim, J. H. Han, M. K. Kim, C. S. Lim, H. M. Kim, B. R. Cho, Angew. Chem. Int. Ed. 2010, 49, 6786-6789.

[269] O. Tour, S. R. Adams, R. A. Kerr, R. M. Meijer, T. J. Sejnowski, R. W. Tsien, R. Y. Tsien, Nat. Chem. Biol. 2007, 3, 423-431.

[270] E. Carafoli, C. B. Klee, Calcium as a Cellular Regulator, Oxford University Press, 1999.

[271] S. Yan, Photoaktivierungsfähige Rhodamine Als Bio-Calcium-Sensoren Und Markierungen Für Tetracystein-Tags in Proteinen, Georg-August-Universität Göttingen, 2011.

[272] K. Lang, J. W. Chin, Chem. Rev. 2014, 114, 4764-4806.

[273] C. Vorndran, A. Minta, M. Poenie, Biophys. J. 1995, 69, 2112-2124.

[274] J. Graf, Synthese von fluoreszierenden Calcium-Sensoren und Biomolekülen zur Untersuchung physiologischer Prozesse, Cuvillier Verlag, 2017.

[275] M. Meldal, C. W. Tornøe, Chem. Rev. 2008, 108, 2952-3015.

[276] V. D. Bock, H. Hiemstra, J. H. van Maarseveen, Eur. J. Org. Chem. 2006, 2006, 51-68.

[277] C. Hansen, Synthesis of Modified and Labelled Lipids for Analysis of Enzyme Mechanisms and Membrane Interactions, 2017.

[278] F. Liepouri, E. Foukaraki, T. G. Deligeorgiev, H. E. Katerinopoulos, Cell Calcium 2001, 30, 331-335.

[279] S. T. Caldwell, A. G. Cairns, M. Olson, S. Chalmers, M. Sandison, W. Mullen, J. G. McCarron, R. C. Hartley, Tetrahedron 2015, 71, 9571-9578. 
[280] X. Ji, B. Cheng, J. Song, C. Liu, Y. Wang, Synth. Commun. 2009, 39, 2053-2057.

[281] A. R. Surrey, Name Reactions in Organic Chemistry, Elsevier Science, Saint Louis, 1961.

[282] A. P. Rajput, P. D. Girase, 2012, 3, 25-43.

[283] S. Kovalenko, I. Bylov, K. Sytnik, V. Chernykh, Y. Bilokin, Molecules 2000, 5 , $1146-1165$.

[284] S. N. Kovalenko, M. V. Vasil'ev, I. V. Sorokina, V. P. Chernykh, A. V. Turov, S.

A. Rudnev, Chem. Heterocycl. Compd. 1998, 34, 1412-1415.

[285] S. V. Ley, A. J. Stewart-Liddon, D. Pears, R. H. Perni, K. Treacher, Beilstein J. Org. Chem. 2006, 2, 15.

[286] T. Deligeorgiev, T. Tsvetkova, D. Ivanova, I. Timtcheva, Color. Technol. 2008, 124, 195-203.

[287] O. V. Khilya, M. S. Frasinyuk, A. V. Turov, V. P. Khilya, Chem. Heterocycl. Compd. 2001, 37, 1029-1037.

[288] B. Roubinet, C. Massif, M. Moreau, F. Boschetti, G. Ulrich, R. Ziessel, P.-Y.

Renard, A. Romieu, Chem. - Eur. J. 2015, 21, 14589-14601.

[289] A. El-Faham, F. Albericio, Chem. Rev. 2011, 111, 6557-6602.

[290] A. Isidro-Llobet, M. Álvarez, F. Albericio, Chem. Rev. 2009, 109, 2455-2504.

[291] E. I. Vrettos, N. Sayyad, E. M. Mavrogiannaki, E. Stylos, A. D. Kostagianni, S. Papas, T. Mavromoustakos, V. Theodorou, A. G. Tzakos, RSC Adv. 2017, 7, 5051950526.

[292] F. Albericio, J. M. Bofill, A. El-Faham, S. A. Kates, J. Org. Chem. 1998, 63, 96789683.

[293] M. Collot, C. Wilms, J.-M. Mallet, RSC Adv. 2014, 5, 6993-7000.

[294] S. Jónsson, C. S. Arribas, O. F. Wendt, J. S. Siegel, K. Wärnmark, Org Biomol Chem 2005, 3, 996-1001.

[295] J. K. Mishra, G. Panda, Synthesis 2005, 2005, 1881-1887.

[296] F. D. Bellamy, K. Ou, Tetrahedron Lett. 1984, 25, 839-842.

[297] E. Roussakis, F. Liepouri, A.-P. Nifli, E. Castanas, T. G. Deligeorgiev, H. E. Katerinopoulos, Cell Calcium 2006, 39, 3-11.

[298] W. C. Chan, P. D. White, Eds., Fmoc Solid Phase Peptide Synthesis: A Practical Approach, Oxford University Press, New York, 2000.

[299] C. G. Sinn, M. Antonietti, R. Dimova, Colloids Surf. Physicochem. Eng. Asp. 2006, 282-283, 410-419.

[300] N. Kaneko, R. Matsuda, M. Toda, K. Shimamoto, Biochim. Biophys. Acta BBA Biomembr. 1997, 1330, 1-7.

[301] J. A. Veiro, P. R. Cullis, Biochim. Biophys. Acta BBA - Biomembr. 1990, 1025, $109-115$.

[302] K. R. Gee, K. A. Brown, W.-N. U. Chen, J. Bishop-Stewart, D. Gray, I. Johnson, Cell Calcium 2000, 27, 97-106.

[303] N. J. Greenfield, Nat. Protoc. 2007, 1, 2876-2890.

[304] Ž. Štirn, A. Ručigaj, M. Krajnc, Polymer 2017, 120, 129-140.

[305] T. Madrakian, F. Fazl, M. Ahmadi, A. Afkhami, New J Chem 2016, 40, 122-129.

[306] C. Y. Ang, S. Y. Tan, C. Teh, J. M. Lee, M. F. E. Wong, Q. Qu, L. Q. Poh, M. Li, Y. Zhang, V. Korzh, et al., Small 2017, 13, 1602379.

[307] B. Kolesinska, K. K. Rozniakowski, J. Fraczyk, I. Relich, A. M. Papini, Z. J.

Kaminski, Eur. J. Org. Chem. 2015, 2015, 401-408.

[308] T. R. Carvajal, S. M. Kuebler, C. A. Sierra, Synth. Met. 2015, 209, 183-187. 


\section{Acknowledgements}

Mein besonderer Dank gilt Prof. Dr. Ulf Diederichsen für die interessante Themenstellung. Die hierbei gewährte wissenschaftliche Freiheit ermöglichte es mir selbstständig den Themengebieten nachzugehen und unsere ideenreichen Diskussionen halfen mir einige Probleme zu lösen. Das in mich gesetzte Vertrauen durch die Übertragung von spannenden Aufgaben, wie z.B. der technischen Betreuung unserer Peptidsynthesizer oder der Betreuung von Bachelorstudenten hat mir geholfen meine Fähigkeiten weiterzuentwickeln.

Ich danke Prof. Dr. Claudia Steinem für die freundliche Übernahme des Korreferats dieser Arbeit. Gerade ihr umfangreiches Wissen im Bereich der Biomembranen ermöglichte es mir die gestellten wissenschaftlichen Fragen von einem anderen Standpunkt zu betrachten. Darüber hinaus möchte ich mich für die Möglichkeit bedanken, meine Promotion im Rahmen des SFB 803 durchzuführen. Gerade durch die fachliche Kompetenz und der guten Organisation war ein reger Austausch mit anderen Wissenschaftlern immer vorhanden.

Darüber hinaus danke ich den weiteren Mitgliedern meiner Prüfungskommission Prof. Dr. Jörg Enderlein, Prof. Dr. Kai Tittmann, Dr. Franziska Thomas und Dr. Holm Frauendorf.

Der NMR-Abteilung unter der Leitung von Herrn R. Machinek \& Herrn Dr. M. John, sowie der Massenabteilung unter der Leitung von Herrn Dr. H. Frauendorf danke ich für die Messung der zahlreichen Spektren sowie für ihren Einsatz.

Meinen jetzigen und ehemaligen Kollegen im AK Diederichsen und besonders denen aus Labor 108, Dr. Ulrike Rost, Markus Wiegand, Martin Kloos, Atida Nasufovska, Dr. Hanna Agnes Radzey, Dr. Julia Schneider, Dr. Janine Wegner, Dr. Marta Anna Cal, Iryna Portnova und Dr. Kanlidere Zeynep möchte ich für die gute und produktive Arbeitsatmosphäre danken.

Ich danke meinen Bachelorstudenten Vivien Schulte (Chem.), Sarmini Nageswaran (Chem.), Rostand Roi Ngouoko (Biochem.), Benjamin Gnoth (Biochem.) und Lennart Carl Langenhoff (Biochem) für ihre Unterstützung in den verschiedenen Themen meiner Arbeit. Durch euch habe ich, neben wissenschaftlichen Erkenntnissen, viel Spaß gehabt und viele wichtige Erfahrung gesammelt. Ebenfalls möchte ich mich bei meinen Abteilungspraktikanten Benjamin Schmidt, Thierry Hartung, Tobias Schmidt und Stefan Lülf bedanken.

Meinen Kooperationspartnern bin ich zu großem Dank verpflichtet, da ohne die interessanten Problemstellungen ein Teil dieser Arbeit nicht möglich gewesen wäre. Hier möchte ich Aditya Satyabodha Katti (AK Enderlein, Göttingen), Prof. Dr. P. Pohl (Linz) und Jana Staffa (AK Hildebrandt, Berlin) nennen. Die hier gezeigten Ergebnisse sind hoffentlich nur der Grundsteine für weitere Studien. 
Für die Finanzierung meiner Arbeit danke ich Prof. Dr. U. Diederichsen, der GeorgAugust-Universität Göttingen und der Deutschen Forschungsgemeinschaft (DFG) im Rahmen des SFB 803 „Funktionalität kontrolliert durch Organisation in und zwischen Membranen“.

Für das Korrekturlesen dieser Arbeit danke ich Yvonne Link, Dr. Ulrike Rost, Markus Wiegand, Martin Kloos, Dr. Barbara Hubrich, Angela Heinemann, Aoife Neville und Patrick Menzel.

Weiter möchte ich mich bei Angela Heinemann und Aoife Neville für die persönliche und die organisatorische Unterstützung bedanken. Das Sekretariat war immer ein Ort in den man mit seinen großen und kleinen Problemen kommen konnte. Daniel Frank möchte ich zum einen für die technische Unterstützung im AK danken und für die unzähligen Liter Diazomethan, die er für uns hergestellt hat.

Darüber hinaus möchte ich allen Mitgliedern des AK Steinem danken. Neben Dr. Ingo Mey und Jutta Gerber-Nolte, welche mir immer eine sichere Anlaufpunkt waren, konnte ich mich auf die anderen Doktoranden verlassen. Es bestand immer die Möglichkeit meine Probleme zu diskutieren. Darüber hinaus haben sie sich auch mehr als einmal die Zeit genommen Messungen mit mir durchzuführen.

Beim C. A. H. und seinen Mitgliedern möchte ich mich für die geschlossenen Freundschaften und die Zerstreuung bedanken, welche ich von euch erhalten habe. Frank D., dir danke ich besonders. Auf dich kann ich immer bauen.

Schließlich danke ich meiner Familie für ihre Unterstützung während meiner Zeit in Göttingen und Yvonne L. möchte ich dafür danken, dass sie so ist wie sie ist und mich auf diesem Weg solange begleitet und unterstützt hat. 
